\title{
Intestinal cell damage, inflammation and wound healing in major gastrointestinal surgery
}

Citation for published version (APA):

Reisinger, K. W. (2015). Intestinal cell damage, inflammation and wound healing in major gastrointestinal surgery. [Doctoral Thesis, Maastricht University]. Datawyse / Universitaire Pers Maastricht. https://doi.org/10.26481/dis.20150605kr

Document status and date:

Published: 01/01/2015

DOI:

10.26481/dis.20150605kr

Document Version:

Publisher's PDF, also known as Version of record

\section{Please check the document version of this publication:}

- A submitted manuscript is the version of the article upon submission and before peer-review. There can be important differences between the submitted version and the official published version of record.

People interested in the research are advised to contact the author for the final version of the publication, or visit the DOI to the publisher's website.

- The final author version and the galley proof are versions of the publication after peer review.

- The final published version features the final layout of the paper including the volume, issue and page numbers.

Link to publication

\footnotetext{
General rights rights.

- You may freely distribute the URL identifying the publication in the public portal. please follow below link for the End User Agreement:

www.umlib.nl/taverne-license

Take down policy

If you believe that this document breaches copyright please contact us at:

repository@maastrichtuniversity.nl

providing details and we will investigate your claim.
}

Copyright and moral rights for the publications made accessible in the public portal are retained by the authors and/or other copyright owners and it is a condition of accessing publications that users recognise and abide by the legal requirements associated with these

- Users may download and print one copy of any publication from the public portal for the purpose of private study or research.

- You may not further distribute the material or use it for any profit-making activity or commercial gain

If the publication is distributed under the terms of Article $25 \mathrm{fa}$ of the Dutch Copyright Act, indicated by the "Taverne" license above, 
(c) Copyright K.W. Reisinger, Maastricht 2015

ISBN 9789461594396

Cover design: Christine Muris

Cover: "What goes on four feet, on two feet, and three, but the more feet it goes on, the weaker it be?" Sophocles - Oedipus Rex

Production: Datawyse Maastricht

Printing of this thesis was financially supported by the Nederlandse Vereniging voor Gastroenterologie.

The studies in this thesis were performed at the Nutrition and Toxicology Research Institute Maastricht (NUTRIM).

All rights reserved. No part of this thesis may be reproduced or transmitted in any form or by any means, electronic or mechanical, including photocopying, recording or any information storage or retrieval system, without permission in writing from the author, or, when appropriate, from the publishers of publications. 


\section{Intestinal cell damage, inflammation and wound healing in major gastrointestinal surgery}

PROEFSCHRIFT

ter verkrijging van de graad van doctor

aan de Universiteit Maastricht,

op gezag van de Rector Magnificus,

Prof. dr. L.L.G. Soete,

volgens het besluit van het College van Decanen,

in het openbaar te verdedigen

op vrijdag 5 juni 2015 om 14:00 uur

door

Kostan Werner Reisinger

geboren op 16 oktober 1985 te Muiden

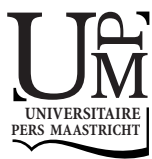




\section{Promotores}

Prof. dr. M.F. von Meyenfeldt

Prof. dr. L.W.E. van Heurn

Copromotor

Dr. M. Poeze

\section{Beoordelingscommissie}

Prof. dr. C.H.C. Dejong (voorzitter)

Dr. N.D. Bouvy

Prof. dr. K.C.H. Fearon, University of Edinburgh

Prof. dr. E. Heineman, Universitair Medisch Centrum Groningen

Dr. K. Lenaerts 
Aan mijn ouders

Voor Aleida, Nina en Max 



\section{Table of contents}

Chapter 1. General introduction

Part 1. Pathophysiologic aspects of complications after adult gastrointestinal surgery

Chapter 2. Doppler guided goal-directed fluid therapy increases intestinal perfusion in colorectal surgery

Chapter 3. Cyclooxygenase-2 is essential for colorectal anastomotic healing

Chapter 4. Functional compromise reflected by sarcopenia, frailty and nutritional depletion predicts adverse postoperative outcome after colorectal cancer surgery

Chapter 5. Sarcopenia is associated with an increased inflammatory response to surgery in colorectal cancer

Chapter 6. Loss of skeletal muscle mass during neoadjuvant therapy predicts postoperative mortality in esophageal cancer surgery

Part 2. Biomarkers of complications after adult gastrointestinal surgery

Chapter 7. Accurate prediction of anastomotic leakage after colorectal surgery using plasma markers for intestinal damage and inflammation

Part 3. Pathophysiologic aspects of necrotizing enterocolitis (NEC)

Chapter 8. Intestinal fatty acid binding protein (I-FABP): a possible marker for gut maturation

Chapter 9. Breast feeding improves gut maturation compared to formula feeding in preterm babies

Part 4. Biomarkers of necrotizing enterocolitis (NEC)

Chapter 10. Non-invasive measurement of fecal calprotectin and serum amyloid A (SAA) combined with intestinal Fatty Acid Binding Protein (I-FABP) in necrotizing enterocolitis (NEC) 
Chapter 11. Non-invasive serum amyloid A (SAA) measurement and plasma platelets for accurate prediction of surgical intervention in severe necrotizing enterocolitis (NEC)

Chapter 12. Non-invasive measurement of intestinal epithelial damage at time of re-feeding can predict clinical outcome after necrotizing enterocolitis 207

Chapter 13. Summary and discussion 221

Valorization 237

Samenvatting 241

Dankwoord 249

Scientific output 261

Curriculum vitae 265 
Chapter 1. General introduction

Partly adapted from: Jeroen L.A. van Vugt, Kostan W. Reisinger, Joep P.M. Derikx, Djamila Boerma, Jan H.M.B. Stoot. Improving outcomes in oncological colorectal surgery. World Journal of Gastroenterology 2014. 20(35):12445-57 


\subsection{Introduction}

Major gastrointestinal surgery is the treatment of choice in a variety of gastrointestinal diseases, such as cancer and severe inflammatory conditions of the gut. This type of surgery carries significant risk of complications, which negatively affects treatment effectiveness, survival, health-related quality of life and healthcare costs ${ }^{1,2}$. Furthermore, gut functioning has a central role in patients recovering from different kinds of insults, such as surgery. First, adequate digestion and absorption of nutrients is necessary to recover from the compromised physical condition imposed by surgery and anesthesia. Second, integrity of the gut barrier is essential to avoid exposure to luminal bacteria and other toxins related to postoperative complications ${ }^{3-5}$. Compromise of these functions may lead to inadequate wound healing, postoperative morbidity and mortality. The search for improvements in perioperative care is therefore of crucial importance and should aim at fundamental aspects, prevention, and diagnosis of impaired gut function and complications.

In this thesis, it is hypothesized that especially in vulnerable patients with gastrointestinal disorders, inadequate wound healing, postoperative morbidity and mortality are likely to develop. To investigate this hypothesis, patients at the extremes of age were primarily investigated, i.e. elderly patients and premature neonates. In paragraph 1.2, opportunities for improvement in adult oncological gastrointestinal surgery are discussed. First, important aspects of vulnerability of elderly patients such as frailty and nutritional compromise are considered in paragraphs 1.2.1 and 1.2.2. Second, clinical and laboratory markers are indispensible tools for timely recognition of postoperative complications, which is described in paragraph 1.2.3. Third, perioperative optimization strategies have led to improved surgical outcome in the past decennia. Several elements of perioperative optimization are however subject to debate, which necessitates further investigation as shown in paragraph 1.2.4. In paragraph 1.3, another vulnerable patient population is considered, namely premature neonates. Necrotizing enterocolitis (NEC) is the most severe gastrointestinal emergency in these patients and one of the primary indications for pediatric gastrointestinal surgery. Possibilities to improve the diagnosis, treatment and pathophysiological knowledge of NEC are discussed. 


\subsection{Oncological gastrointestinal surgery}

Colorectal cancer is one of the predominant types of cancer and the fourth leading cause of cancer-associated deaths worldwide ${ }^{6}$. In numbers: 600,000 patients died of colorectal cancer in 2008 worldwide, and disability-adjusted life-years lost from colorectal cancer were 300 per 100,000 patients, which was estimated to be $7 \%$ of the total cancer burden worldwide ${ }^{7}$. Sixty-six per cent of patients with colorectal cancer will undergo at least one major surgical resection 8. The perioperative course of colorectal surgery for malignancy is crucial for the clinical outcome of treatment, in terms of mortality, tolerance, efficacy, and functional recovery, and has a considerable impact on health care resources ${ }^{9,10}$. In the past decennia, perioperative care has improved largely due to advances in anesthetic and analgesic approaches, minimally invasive operative techniques, and the introduction of fast-track protocols ${ }^{11,12}$. Despite these improvements, many complications are still observed after oncological colorectal surgery, leading to prolonged hospital stay and high readmission rates with concurrent health care costs ${ }^{2}$. Early recognition and adequate intervention of complications will attenuate severity and may eventually prevent mortality.

Anastomotic leakage is among the most prevalent and detrimental complications of colorectal surgery and is a severe form of gut function disruption. Of 10,017 registered resections for colorectal cancer in the Netherlands in 2012, 691 (6.9\%) were complicated by anastomotic leakage requiring re-intervention (Dutch Surgical Colorectal Audit 2012) ${ }^{13}$, making anastomotic leakage the primary complication requiring re-intervention. In high-risk patients incidence rates can even increase to $18 \%{ }^{14}$. Anastomotic leakage is associated with high morbidity ${ }^{15}$, mortality ${ }^{16}$, reoperation rates ${ }^{10}$, and duration of hospitalization ${ }^{17}$. In cancer, anastomotic leakage is related to diminished disease-specific survival and higher recurrence rates ${ }^{1,10,18}$. It is therefore imperative to find new strategies to prevent, diagnose and treat anastomotic leakage, or implement what is already known.

\subsubsection{Frailty and sarcopenia}

Advanced age is associated with an increased incidence of cancer ${ }^{19}$. The number of elderly cancer patients is concomitantly increasing. Fifty per cent of patients with colorectal cancer is above the age of 70 years ${ }^{13}$. While survival of all cancer types is increasing, the improvement of cancer outcome in older patients is relatively limited ${ }^{20}$. Higher age is an independent predictor of disease-specific 
perioperative mortality in patients undergoing surgery for colorectal cancer ${ }^{21,22}$. Weight loss, cachexia and nutritional compromise, especially in older patients, are associated with impaired response to chemotherapy and decreased survival ${ }^{23,24}$.

Frail elderly undergoing colorectal surgery have a 4-fold increased risk of major postoperative complications ${ }^{25}$. Frailty is a state of increased vulnerability towards stressors in older individuals, leading to an increased risk of developing adverse health outcomes ${ }^{26}$. The definitions and biological characteristics of frailty are subject to debate. Weight loss, decreased muscle strength, reduced physical activity, exhaustion, and reduced walking speed are symptoms of a physical definition of frailty ${ }^{25,27}$, whereas comorbidity, polypharmacy, decreased physical functioning, impaired nutritional and cognitive status, depression and social support are components of a more multidimensional description of frailty ${ }^{28}$. A simple screening instrument for frailty is the Groningen Frailty Index (GFI), based on physical, cognitive, social and emotional items ${ }^{29}$. Skeletal muscle depletion or sarcopenia is an element of frailty in both definitions. Sarcopenia, which can easily be assessed by measurement of muscle area at the level of the third lumbar spine at CT-scan, is associated with prolonged hospital stay, infectious complications and decreased recurrence and survival rates following colorectal and liver surgery ${ }^{30,31}$. However, a relationship with anastomotic leakage has not yet been established.

\subsubsection{Nutritional status}

A powerful and easily obtainable tool to assess the patient's physical and/or mental condition before operation is the use of questionnaires. Various questionnaires have been developed to evaluate nutritional status. A poor nutritional condition correlates well with impaired quality of life and physical functioning ${ }^{32}$. The Short Nutritional Assessment Questionnaire (SNAQ) and Malnutrition Universal Screening Tool (MUST) scores are commonly used nutritional screening tools in surgical patients. These questionnaires accurately detect malnutrition, and the MUST score predicts postoperative complications in cardiac surgery ${ }^{33}$. Evidence for the value of nutritional screening tools to predict postoperative outcome in colorectal surgery is lacking. As one in five patients undergoing colorectal surgery is malnourished ${ }^{34}$, the detection of nutritional depletion is of great importance, especially with neo-adjuvant therapies which can further compromise the nutritional and metabolic status. A misbalance between energy expenditure and nutritional supplementation, combined with metabolic inefficiency leading 
to protein breakdown particulary in muscle are the fundamental physiologic derangements leading to cancer-induced weight loss. The combination of energy imbalance and metabolic derangements are associated with poor clinical outcome after surgery ${ }^{35,36}$. This may at least partly be related to impaired wound healing when metabolic substrates are depleted.

Although nutritional supplementation strategies in oncological colorectal surgery can improve handgrip strength, pulmonary function and insulin resistance ${ }^{37}$, nutritional support has not been proven unequivocally effective to reduce length of hospital stay and anastomotic leakage rates ${ }^{38}$. It may be concluded that only severely malnourished patients benefit from nutritional support ${ }^{39,40}$. Nutritional status questionnaires may however not only be used to identify malnourished patients for nutritional support. As a tool for accurate prediction of postoperative complications, SNAQ and MUST scores could lead the way to other treatment options, for example surgery without a primary anastomosis or protection of the anastomosis using a diverting stoma. The predictive value of these scores for postoperative complications remains yet to be determined.

\subsubsection{Clinical and laboratory detection of anastomotic leakage}

The clinical presentation of anastomotic leakage is heterogeneous and often nonspecific. Anastomotic leakage is therefore frequently diagnosed late ${ }^{41}$. Furthermore, some leaks develop subclinically and are only detectable with radiological examination. Abdominal CT scan with intraluminal contrast has considerably improved timely recognition of anastomotic leakage, although it yields low sensitivity (68\%), which may delay the diagnosis and appropriate treatment ${ }^{42}$. Delay in recognizing and consequently treating anastomotic leakage after colorectal surgery is associated with increased mortality ${ }^{43,44}$.

Clinical signs for accurate and early detection of anastomotic leakage have been widely investigated. Den Dulk and colleagues standardized postoperative monitoring and developed a leakage-score, consisting of general, local physical examination, laboratory investigation and dietary items. The use of this score resulted in a significantly shorter delay in the diagnosis of anastomotic leakage ${ }^{45}$.

Accurate diagnostic markers are needed to detect anastomotic leakage early after colorectal surgery. Various biomarkers have been investigated, although none has been validated clinically and studies are difficult to compare, mainly due to different definitions of anastomotic leakage ${ }^{46}$. C-reactive protein (CRP) has been widely proposed as an early indicator to diagnose anastomotic leakage 
on postoperative day $2-4{ }^{47}$. However, the test characteristics are not convincingly robust, with approximately $70-80 \%$ sensitivity and specificity ${ }^{47}$. Currently, the PRECIOUS trial investigates a step-up approach in major abdominal surgery combining CRP and CT imaging of the abdomen to diagnose severe complications, including anastomotic leakage ${ }^{48}$. Specific plasma markers for intestinal cell damage and inflammation may provide better accuracy.

\subsubsection{Perioperative optimization strategies}

Several meta-analyses have shown that Enhanced Recovery After Surgery (ERAS) programs result in reduced length of hospital stay and overall complications without affecting patient safety 12, 49,50. Although strong evidence exists for many recommendations, such as antibiotic prophylaxis and preoperative bowel preparation, controversies remain around perioperative fluid therapy and use of non-steroidal anti-inflammatory drugs (NSAIDs) ${ }^{39,51 .}$

To reduce cardiopulmonary complications, restrictive fluid regimens seem superior to liberal fluid treatment ${ }^{52}$. Both liberal and restrictive fluid therapies may induce hypoperfusion of the anastomosis by causing local edema or hypovolemia, which could be avoided by individualized, goal-directed fluid therapy, aiming at maximal stroke volumes. A recent randomized controlled trial (RCT) using esophageal Doppler monitoring for cardiac output measurement could however not prove a reduction in postoperative complications ${ }^{53}$. The benefits of goaldirected fluid therapy in colorectal surgery are not indisputable and the effects on intestinal integrity are not known. Therefore, the effect of goal-directed fluid therapy on intestinal perfusion, damage and healing needs further exploration.

Several animal and human studies have indicated that the use of NSAIDs is markedly correlated with anastomotic leakage following colorectal surgery ${ }^{54-}$ 56. The ERAS guidelines state that sufficient evidence is lacking to stop using NSAIDs as a component of multimodal analgesia ${ }^{39}$. The mechanisms by which NSAIDs exert their detrimental effects on colonic surgical wound healing are not known, which deserves further in-depth investigation. As classical wound healing, anastomotic wound healing consists of inflammation, proliferation, and remodeling of which the first two phases are principally of interest in the context of anastomotic leakage, which develops within several days after surgery 57. Platelets first establish hemostasis, followed by influx of inflammatory cells, mainly neutrophils, monocytes and macrophages ${ }^{58}$. Myofibroblasts, regulated by various growth factors, then create collagen-rich granulation tissue, a process 
in which angiogenesis plays a crucial role. Cyclooxygenase-2 (COX-2), the ratelimiting enzyme in the conversion of arachidonic acid into various prostaglandins, is involved in inflammation, proliferation and angiogenesis ${ }^{59-61}$. Since NSAIDs block COX-2, these mechanisms can be involved in the detrimental effects of NSAIDs on gastrointestinal wound healing and should therefore be investigated.

\subsection{Pediatric gastrointestinal surgery}

Necrotizing enterocolitis (NEC) is the most frequent indication for gastrointestinal surgery in infants, affecting predominantly premature neonates ${ }^{62}$. NEC carries high morbidity and mortality $(20-40 \%)^{63}$. Early diagnosis, the detection of severe NEC requiring surgical treatment, and the timing to reintroduce enteral feeding after NEC episodes are unresolved issues. The initial clinical manifestations of NEC are non-specific and indistinguishable from other gastrointestinal disorders and non-abdominal sepsis ${ }^{64}$. The diagnosis is further hampered by limited diagnostic accuracy of laboratory tests and currently used imaging modalities ${ }^{65}$, ${ }^{66}$. Therefore, there is a high need for accurate tests to diagnose NEC and severe NEC necessitating surgery at an early stage.

Furthermore, knowledge of the pathophysiological processes underlying NEC is required. The etiology of NEC remains poorly understood, although a strong correlation with formula feeding has been established ${ }^{67}$. Growth factors abundantly present in human milk, including epidermal growth factor and insulin-like growth factor, which stimulate epithelial cell growth and cell differentiation, are supposed to play an important role in the regulation of the neonatal gastrointestinal development 68,69 . The effect of human milk on intestinal damage and maturation should be further investigated to extend knowledge on the mechanisms by which human milk is beneficial, which may ultimately lead to finding appropriate human milk substitutes. Furthermore, NEC incidence is inversely associated with gestational age ${ }^{70}$. Intestinal barrier function is incompletely developed in the first week after birth indicated by higher intestinal permeability, especially in premature infants ${ }^{71-75}$. Immaturity of the gut also plays a pivotal role in the development of gut-derived sepsis and feeding problems ${ }^{76}$. The underlying mechanisms remain poorly understood, but increased intestinal permeability and inadequate intestinal immune responses in preterm neonates are associated with gastrointestinal disorders ${ }^{74,77,78}$. New research should aim at defining intestinal morphological differences between premature and term infants, and differences 
in circulating levels of gut-specific markers to obtain better understanding of the immature gut. This may provide necessary knowledge for the development of protective strategies in this vulnerable patient population.

\subsection{Aims to be studied}

As outlined in this chapter, various opportunities exist to improve outcomes in gastrointestinal surgery. The central hypothesis of this thesis was that in vulnerable patients with gastrointestinal disorders, inadequate wound healing, postoperative morbidity and mortality are likely to develop. Therefore, patients at the extremes of age, i.e. elderly patients and premature neonates with severe gastrointestinal morbidity were investigated. The aims of this thesis were to:

- study factors and mechanisms underlying intestinal cell damage, inflammation and impaired wound healing in gastrointestinal surgery to find new strategies for the prevention of postoperative complications (Chapter 2-6, Pathophysiologic aspects of complications after adult gastrointestinal surgery)

- investigate biomarkers to detect complications early after colorectal surgery (Chapter 7, Biomarkers of complications after adult gastrointestinal surgery)

- characterize gut-specific biomarkers in the context of gut maturation to gain better understanding of the pathophysiology of NEC (Chapter 8-9, Pathophysiologic aspects of NEC)

- assess the usefulness of gut-specific biomarkers in the detection, treatment and follow-up of pediatric surgery for NEC (Chapter 10-12, Biomarkers of NEC) 


\section{References}

1. Krarup PM, Nordholm-Carstensen A, Jorgensen LN, et al. Anastomotic Leak Increases Distant Recurrence and Long-Term Mortality After Curative Resection for Colonic Cancer: A Nationwide Cohort Study. Ann Surg 2013.

2. Wick EC, Shore AD, Hirose K, et al. Readmission rates and cost following colorectal surgery. Dis Colon Rectum 2011;54:1475-9.

3. Deitch EA. Multiple organ failure. Pathophysiology and potential future therapy. Ann Surg 1992;216:117-34.

4. Holland J, Carey M, Hughes N, et al. Intraoperative splanchnic hypoperfusion, increased intestinal permeability, down-regulation of monocyte class II major histocompatibility complex expression, exaggerated acute phase response, and sepsis. Am J Surg 2005;190:393-400.

5. Swank GM, Deitch EA. Role of the gut in multiple organ failure: bacterial translocation and permeability changes. World J Surg 1996;20:411-7.

6. Garcia-Granero A, Frasson M, Flor-Lorente B, et al. Procalcitonin and C-reactive protein as early predictors of anastomotic leak in colorectal surgery: a prospective observational study. Dis Colon Rectum 2013;56:475-83.

7. Soerjomataram I, Lortet-Tieulent J, Parkin DM, et al. Global burden of cancer in 2008: a systematic analysis of disability-adjusted life-years in 12 world regions. Lancet 2012;380:1840-50.

8. National Cancer Intelligence Network: major surgical resections report 2011.

9. Law WL, Choi HK, Lee YM, et al. The impact of postoperative complications on longterm outcomes following curative resection for colorectal cancer. Ann Surg Oncol 2007;14:2559-66.

10. Walker KG, Bell SW, Rickard MJ, et al. Anastomotic leakage is predictive of diminished survival after potentially curative resection for colorectal cancer. Ann Surg 2004;240:255-9.

11. Kehlet H. Fast-track colorectal surgery. Lancet 2008;371:791-3.

12. Zhuang $\mathrm{CL}$, Ye $X Z$, Zhang $X D$, et al. Enhanced recovery after surgery programs versus traditional care for colorectal surgery: a meta-analysis of randomized controlled trials. Dis Colon Rectum 2013;56:667-78.

13. Jaarrapportage 2012: uitkomst van zorg registratie. Dutch Surgical Colorectal Audit, 2012.

14. Tan WP, Talbott VA, Leong QQ, et al. American Society of Anesthesiologists class and Charlson's comorbidity index as predictors of postoperative colorectal anastomotic leak: a single-institution experience. J Surg Res 2013;184:115-9.

15. Makela JT, Kiviniemi H, Laitinen S. Risk factors for anastomotic leakage after left-sided colorectal resection with rectal anastomosis. Dis Colon Rectum 2003;46:653-60.

16. Snijders HS, Wouters MW, van Leersum NJ, et al. Meta-analysis of the risk for anastomotic leakage, the postoperative mortality caused by leakage in relation to the overall postoperative mortality. Eur J Surg Oncol 2012;38:1013-9. 
17. Golub R, Golub RW, Cantu R, Jr., et al. A multivariate analysis of factors contributing to leakage of intestinal anastomoses. J Am Coll Surg 1997;184:364-72.

18. Mirnezami A, Mirnezami R, Chandrakumaran K, et al. Increased local recurrence and reduced survival from colorectal cancer following anastomotic leak: systematic review and meta-analysis. Ann Surg 2011;253:890-9.

19. DePinho RA. The age of cancer. Nature 2000;408:248-54.

20. Berrino F, Verdecchia A, Lutz JM, et al. Comparative cancer survival information in Europe. Eur J Cancer 2009;45:901-8.

21. McMillan DC, McArdle CS, Morrison DS. A clinical risk score to predict 3-, 5- and 10year survival in patients undergoing surgery for Dukes B colorectal cancer. Br J Cancer 2010;103:970-4.

22. Matthiessen P, Hallbook O, Rutegard J, et al. Population-based study of risk factors for postoperative death after anterior resection of the rectum. Br J Surg 2006;93:498503.

23. Schiesser M, Kirchhoff $\mathrm{P}$, Muller MK, et al. The correlation of nutrition risk index, nutrition risk score, and bioimpedance analysis with postoperative complications in patients undergoing gastrointestinal surgery. Surgery 2009;145:519-26.

24. Persson C, Glimelius B. The relevance of weight loss for survival and quality of life in patients with advanced gastrointestinal cancer treated with palliative chemotherapy. Anticancer Res 2002;22:3661-8.

25. Tan KY, Kawamura YJ, Tokomitsu A, et al. Assessment for frailty is useful for predicting morbidity in elderly patients undergoing colorectal cancer resection whose comorbidities are already optimized. Am J Surg 2012;204:139-43.

26. Fried LP, Hadley EC, Walston JD, et al. From bedside to bench: research agenda for frailty. Sci Aging Knowledge Environ 2005;2005:pe24.

27. Makary MA, Segev DL, Pronovost PJ, et al. Frailty as a predictor of surgical outcomes in older patients. J Am Coll Surg 2010;210:901-8.

28. Saxton A, Velanovich V. Preoperative frailty and quality of life as predictors of postoperative complications. Ann Surg 2011;253:1223-9.

29. Schuurmans $H$, Steverink N, Lindenberg $S$, et al. Old or frail: what tells us more? J Gerontol A Biol Sci Med Sci 2004;59:M962-5.

30. Lieffers JR, Bathe OF, Fassbender K, et al. Sarcopenia is associated with postoperative infection and delayed recovery from colorectal cancer resection surgery. Br J Cancer 2012;107:931-6.

31. van Vledder MG, Levolger S, Ayez N, et al. Body composition and outcome in patients undergoing resection of colorectal liver metastases. Br J Surg 2012;99:550-7.

32. Kruizenga HM, de Jonge $P$, Seidell JC, et al. Are malnourished patients complex patients? Health status and care complexity of malnourished patients detected by the Short Nutritional Assessment Questionnaire (SNAQ). Eur J Intern Med 2006;17:18994.

33. Lomivorotov VV, Efremov SM, Boboshko VA, et al. Evaluation of nutritional screening tools for patients scheduled for cardiac surgery. Nutrition 2013;29:436-42. 
34. Burden ST, Hill J, Shaffer JL, et al. Nutritional status of preoperative colorectal cancer patients. J Hum Nutr Diet 2010;23:402-7.

35. Correia MI, Waitzberg DL. The impact of malnutrition on morbidity, mortality, length of hospital stay and costs evaluated through a multivariate model analysis. Clin Nutr 2003;22:235-9.

36. Deslauriers J, Ginsberg RJ, Dubois P, et al. Current operative morbidity associated with elective surgical resection for lung cancer. Can J Surg 1989;32:335-9.

37. Lidder $\mathrm{P}$, Thomas $\mathrm{S}$, Fleming $\mathrm{S}$, et al. A randomized placebo controlled trial of preoperative carbohydrate drinks and early postoperative nutritional supplement drinks in colorectal surgery. Colorectal Dis 2013;15:737-45.

38. Oguz M, Kerem M, Bedirli A, et al. L-alanin-L-glutamine supplementation improves the outcome after colorectal surgery for cancer. Colorectal Dis 2007;9:515-20.

39. Gustafsson UO, Scott MJ, Schwenk W, et al. Guidelines for perioperative care in elective colonic surgery: Enhanced Recovery After Surgery (ERAS(R)) Society recommendations. Clin Nutr 2012;31:783-800.

40. Bozzetti F, Gavazzi C, Miceli R, et al. Perioperative total parenteral nutrition in malnourished, gastrointestinal cancer patients: a randomized, clinical trial. JPEN J Parenter Enteral Nutr 2000;24:7-14.

41. Hyman N, Manchester TL, Osler T, et al. Anastomotic leaks after intestinal anastomosis: it's later than you think. Ann Surg 2007;245:254-8.

42. Kornmann VN, Treskes N, Hoonhout LH, et al. Systematic review on the value of CT scanning in the diagnosis of anastomotic leakage after colorectal surgery. Int J Colorectal Dis 2012.

43. Alves A, Panis Y, Trancart D, et al. Factors associated with clinically significant anastomotic leakage after large bowel resection: multivariate analysis of 707 patients. World J Surg 2002;26:499-502.

44. Macarthur DC, Nixon SJ, Aitken RJ. Avoidable deaths still occur after large bowel surgery. Scottish Audit of Surgical Mortality, Royal College of Surgeons of Edinburgh. Br J Surg 1998;85:80-3.

45. den Dulk M, Noter SL, Hendriks ER, et al. Improved diagnosis and treatment of anastomotic leakage after colorectal surgery. Eur J Surg Oncol 2009;35:420-6.

46. Hirst N, Tiernan J, Millner P, et al. Systematic review of methods to predict and detect anastomotic leakage in colorectal surgery. Colorectal Dis 2013.

47. Platt JJ, Ramanathan ML, Crosbie RA, et al. C-reactive Protein as a Predictor of Postoperative Infective Complications after Curative Resection in Patients with Colorectal Cancer. Ann Surg Oncol 2012;19:4168-77.

48. Riddez L, Hahn RG, Brismar B, et al. Central and regional hemodynamics during acute hypovolemia and volume substitution in volunteers. Crit Care Med 1997;25:635-40.

49. Varadhan KK, Neal KR, Dejong $\mathrm{CH}$, et al. The enhanced recovery after surgery (ERAS) pathway for patients undergoing major elective open colorectal surgery: a metaanalysis of randomized controlled trials. Clin Nutr 2010;29:434-40. 
50. Spanjersberg WR, Reurings J, Keus F, et al. Fast track surgery versus conventional recovery strategies for colorectal surgery. Cochrane Database Syst Rev 2011:CD007635.

51. Nygren J, Thacker J, Carli F, et al. Guidelines for perioperative care in elective rectal/ pelvic surgery: Enhanced Recovery After Surgery (ERAS(R)) Society recommendations. Clin Nutr 2012;31:801-16.

52. Rahbari NN, Zimmermann JB, Schmidt T, et al. Meta-analysis of standard, restrictive and supplemental fluid administration in colorectal surgery. Br J Surg 2009;96:33141.

53. Brandstrup B, Svendsen PE, Rasmussen M, et al. Which goal for fluid therapy during colorectal surgery is followed by the best outcome: near-maximal stroke volume or zero fluid balance? British journal of anaesthesia 2012;109:191-9.

54. Gorissen KJ, Benning D, Berghmans T, et al. Risk of anastomotic leakage with nonsteroidal anti-inflammatory drugs in colorectal surgery. Br J Surg 2012;99:721-7.

55. Holte K, Andersen J, Jakobsen DH, et al. Cyclo-oxygenase 2 inhibitors and the risk of anastomotic leakage after fast-track colonic surgery. Br J Surg 2009;96:650-4.

56. Klein M, Gogenur I, Rosenberg J. Postoperative use of non-steroidal anti-inflammatory drugs in patients with anastomotic leakage requiring reoperation after colorectal resection: cohort study based on prospective data. BMJ 2012;345:e6166.

57. Witte MB, Barbul A. General principles of wound healing. Surg Clin North Am 1997;77:509-28.

58. Thompson SK, Chang EY, Jobe BA. Clinical review: Healing in gastrointestinal anastomoses, part I. Microsurgery 2006;26:131-6.

59. Buchanan FG, Wang D, Bargiacchi F, et al. Prostaglandin E2 regulates cell migration via the intracellular activation of the epidermal growth factor receptor. J Biol Chem 2003;278:35451-7.

60. Fukata M, Chen A, Klepper A, et al. Cox-2 is regulated by Toll-like receptor-4 (TLR4) signaling: Role in proliferation and apoptosis in the intestine. Gastroenterology 2006;131:862-77.

61. Binion DG, Otterson MF, Rafiee P. Curcumin inhibits VEGF-mediated angiogenesis in human intestinal microvascular endothelial cells through COX-2 and MAPK inhibition. Gut 2008;57:1509-17.

62. Lin PW, Nasr TR, Stoll BJ. Necrotizing enterocolitis: recent scientific advances in pathophysiology and prevention. Semin Perinatol 2008;32:70-82.

63. Neu J, Walker WA. Necrotizing enterocolitis. N Engl J Med 2011;364:255-64.

64. Fanaroff AA, Korones SB, Wright LL, et al. Incidence, presenting features, risk factors and significance of late onset septicemia in very low birth weight infants. The National Institute of Child Health and Human Development Neonatal Research Network. Pediatr Infect Dis J 1998;17:593-8.

65. Hallstrom M, Koivisto AM, Janas $M$, et al. Laboratory parameters predictive of developing necrotizing enterocolitis in infants born before 33 weeks of gestation. J Pediatr Surg 2006;41:792-8. 
66. Tam AL, Camberos A, Applebaum H. Surgical decision making in necrotizing enterocolitis and focal intestinal perforation: predictive value of radiologic findings. J Pediatr Surg 2002;37:1688-91.

67. Lucas A, Cole TJ. Breast milk and neonatal necrotising enterocolitis. Lancet 1990;336:1519-23.

68. Carver JD, Barness LA. Trophic factors for the gastrointestinal tract. Clin Perinatol 1996;23:265-85.

69. Xu RJ. Development of the newborn GI tract and its relation to colostrum/milk intake: a review. Reprod Fertil Dev 1996;8:35-48.

70. Ostlie DJ, Spilde TL, St Peter SD, et al. Necrotizing enterocolitis in full-term infants. J Pediatr Surg 2003;38:1039-42.

71. Israel EJ. Neonatal necrotizing enterocolitis, a disease of the immature intestinal mucosal barrier. Acta Paediatr Suppl 1994;396:27-32.

72. Weaver LT, Laker MF, Nelson R. Intestinal permeability in the newborn. Arch Dis Child 1984;59:236-41.

73. Beach RC, Menzies IS, Clayden GS, et al. Gastrointestinal permeability changes in the preterm neonate. Arch Dis Child 1982;57:141-5.

74. Rouwet EV, Heineman E, Buurman WA, et al. Intestinal permeability and carriermediated monosaccharide absorption in preterm neonates during the early postnatal period. Pediatr Res 2002;51:64-70.

75. van Elburg RM, Fetter WP, Bunkers CM, et al. Intestinal permeability in relation to birth weight and gestational and postnatal age. Arch Dis Child Fetal Neonatal Ed 2003;88:F52-5.

76. Neu J. Gastrointestinal development and meeting the nutritional needs of premature infants. Am J Clin Nutr 2007;85:629S-634S.

77. Neu J, Chen M, Beierle E. Intestinal innate immunity: how does it relate to the pathogenesis of necrotizing enterocolitis. Semin Pediatr Surg 2005;14:137-44.

78. Lin PW, Nasr TR, Stoll BJ. Necrotizing enterocolitis: recent scientific advances in pathophysiology and prevention. Semin Perinatol 2008;32:70-82. 



\section{Part 1 \\ Pathophysiologic aspects of complications after adult gastrointestinal surgery}

The firstaim was to study factors and mechanisms underlying intestinal cell damage, inflammation and impaired wound healing in gastrointestinal surgery to find new strategies for the prevention of postoperative complications. In the first study, it was investigated whether optimization of perioperative fluid administration based on cardiac stroke volumes led to increased splanchnic perfusion and diminished intestinal cell damage in patients undergoing colorectal surgery (chapter 2). In an animal model of colorectal anastomotic leakage, the fundamental role of cyclooxygenase-2 (COX-2) in intestinal wound healing was shown (chapter 3). Next, several aspects of functional compromise and their relationship to postoperative morbidity, mortality and inflammation were investigated in patients undergoing major gastrointestinal surgery (chapters 4-6). Hemodynamic optimization may be of high clinical importance, as a sufficient supply of oxygen and nutritions is needed to ensure adequate wound healing capacity. Furthermore, an important group of analgesics that are frequently used in major surgery inhibit COX-2, and possibly deteriorate postoperative wound healing. Finally, elderly patients are more likely to develop complications, which may be explained by loss of physiologic reserves. Preoperative selection of patients who are at high risk for developing postoperative complications is crucial for therapeutic decisions and for the selection of patients who require preoperative optimization. 

Chapter 2. Doppler guided goal-directed fluid therapy increases intestinal perfusion in colorectal surgery

Kostan W. Reisinger, Henriette M. Willigers, Jochen Jansen, Maarten F. Von Meyenfeldt, Geerard L. Beets, Martijn Poeze. Submitted 


\begin{abstract}
Background

Individualized, goal-directed fluid therapy (GDFT) based on Doppler measurements of stroke volume has been proposed as a treatment strategy in terms of reducing complications, mortality and length of hospital stay in major bowel surgery. The effect of Doppler guided GDFT on intestinal damage and perfusion is studied.
\end{abstract}

\title{
Methods
}

Patients undergoing elective colorectal resection for malignancy were randomized to standard intra- and postoperative fluid therapy with or without additional Doppler guided GDFT. The primary outcome was intestinal epithelial cell damage measured by plasma levels of intestinal fatty acid binding protein (I-FABP). Global intestinal perfusion was measured by gastric tonometry expressed as regional (gastric) minus arterial $\mathrm{CO}_{2}$-gap $\left(\mathrm{P}_{\mathrm{r}-\mathrm{a}} \mathrm{CO}_{2}\right.$-gap).

\section{Results}

I-FABP levels were not different between the intervention group and the control group. Mean areas under the curve (AUCs) of intraoperative $P_{r-a} \mathrm{CO}_{2}$-gaps were significantly lower in the intervention group compared to the control group $(p=0.01)$, indicating better global gastrointestinal perfusion in the intervention group. Moreover, the mean intraoperative $\mathrm{P}_{\mathrm{r}-\mathrm{a}} \mathrm{CO}_{2}$-gap peak in the intervention group was 0.5 (1.0) $\mathrm{kPa}$, which was significantly lower than the mean peak in the control group; 1.4 (1.4) $\mathrm{kPa}, p=0.03$.

\section{Conclusion}

Doppler guided GDFT during and after elective colorectal surgery for malignancy increases global gastrointestinal perfusion. 


\section{Introduction}

Perioperative fluid management in colorectal surgery is subject to debate. Although restrictive fluid regimens seem superior to liberal fluid treatment ${ }^{1}$, euvolemia may be the golden mean. However, euvolemia has not been defined thus far, at least not in terms of generalized amounts of fluid to be administered. Individualized, goal-directed fluid therapy (GDFT) can be considered as closest to euvolemia. GDFT has been proposed as a treatment strategy in terms of reducing complications, mortality and length of hospital stay in major bowel surgery ${ }^{1-6}$. Studies indicate that GDFT is associated with shortened length of hospital stay of 2-3 days compared to controls, and a reduction of major complications requiring intensive care admission, and gastrointestinal complications ${ }^{3,4}$. Typically, goaldirected fluid protocols are designed to optimize stroke volumes intra-operatively guided by oesophageal Doppler monitoring and titration of colloid fluid boluses ${ }^{7,8}$.

The effect of GDFT on intestinal perfusion and subsequently on intestinal damage and wound healing is unknown. Furthermore, the effect of GDFT in the early postoperative period has not been investigated thus far. It is suggested that Doppler guided fluid optimization increases bowel perfusion ${ }^{9}$, while liberal and restrictive treatment strategies may induce hypoperfusion by local oedema and hypovolemia, respectively ${ }^{10}$. Fluid management in the first hours following surgery may be as important as intra-operative fluid management in improving tissue perfusion and oxygenation ${ }^{11}$. In this period, hypovolemia may critically compromise perfusion ${ }^{12}$. In major bowel surgery, this may lead to the development of gutassociated complications, i.e. anastomotic leakage, intra-abdominal abscess and sepsis. GDFT may therefore also be related to a decreased risk on gut-associated complications. However, two recent randomized controlled trials could not prove a reduction in postoperative complications in general when GDFT was compared to restrictive fluid management ${ }^{13,14}$.

The aim of the current study was to investigate as a clinical proof of concept whether oesophageal Doppler guided GDFT approximates euvolemia by attenuating intestinal damage and improving gastrointestinal perfusion during colorectal surgery and during the first hours after colorectal surgery, compared to standard fluid therapy. It was hypothesized that GDFT decreased intestinal injury and improved gastrointestinal perfusion. 


\section{Methods}

\section{Patients}

Fifty-eight patients undergoing colorectal resection for malignancy were enrolled in this single-centre, parallel randomized clinical trial (ClinicalTrials.gov identification number: NCT01175317) between July 2010 and August 2013. Patients were eligible for inclusion if they met the following criteria: elective colorectal cancer surgery with primary anastomosis and a minimum age of 18 years. Written informed consent was obtained from all enrolled patients. Exclusion criteria were: non-malignant causes of intestinal damage (e.g. inflammatory bowel diseases, occlusive disease); use of steroids; history of oesophageal varices and other oesophageal disease; and aortic valve disease. History of oesophageal varices is a contraindication for the use of oesophageal Doppler monitoring and aortic valve disease results in unreliable Doppler measurements. Before randomization, patients were stratified according to the type of surgery, i.e. laparoscopy or open surgery. For allocation of the participants, two computer-generated lists (for laparoscopy and open surgery, respectively) of random numbers were used following a simple randomization to one of two treatment groups. An investigator who did not take part in patient enrolment nor data acquisition was in charge of these lists. The investigator responsible for patient enrolment, Doppler measurements and fluid optimization protocol telephoned the investigator in charge of the lists after obtaining informed consent. The only other person aware of each patients' allocation was the anaesthetist responsible for the perioperative fluid management. The study was approved by the medical ethical committee of Maastricht University Medical Centre, with number 09-2-089 and conducted according to the revised version of the Declaration of Helsinki (October 2008, Seoul). The study methods were not changed after trial registration.

\section{Anaesthetic procedure}

Anaesthesia was induced using propofol, sufentanil, and rocuronium and maintained using sevoflurane. In the majority of patients, an epidural catheter was inserted for additional analgesia using bupivacaine. The epidural catheter was placed at Th8-10, tested with 3-5 mL of 0.25\% bupivacaine with adrenaline, and continuous infusion of bupivacaine $0.25 \%$ was given for sufficient block. After induction of anaesthesia, a medically qualified investigator (K.R.) inserted a oesophageal Doppler probe (CardioQ, Deltex Medical, Chichester, UK) transnasally. 
In both the intervention and control group, oesophageal Doppler measurements were performed every 15 minutes during surgery. The anaesthesiologist was blinded for Doppler measurements at all times. The optimal Doppler signal was obtained according to manufacturers' instructions and stroke volume measurements were averaged over 5 beats. All patients received a radial artery line. Prophylactic antibiotics were given to all patients using metronidazole and cefazoline as guided by protocol.

\section{Fluid treatment and study intervention}

All patients were allowed to drink clear fluids until $2 \mathrm{~h}$ before surgery. Immediately after induction of anaesthesia, stroke volume measurements were performed in all patients. In all patients, Voluven was used to replace blood loss volume in a 1:1 ratio. Voluven and Ringer lactate were infused to maintain the mean arterial pressure (MAP) above $65 \mathrm{mmHg}$. Erythrocyte concentrates were given to keep haemoglobin levels above 5-6 mmol/L depending on age and presence of cardiac disease. If the blood loss was large, plasma and thrombocytes were added. Ephedrine or phenylephrine were given if hypotension persisted despite fluid infusion. In case the inotropic support was needed over a longer time period, noradrenaline was given as continuous infusion.

In the intervention group, standard fluid therapy was as described above. Furthermore, a fluid optimization protocol adapted from Wakeling and colleagues was applied ${ }^{4}$. Immediately after induction of surgery, a $250 \mathrm{~mL}$ bolus of colloid fluid (Voluven) was administered. If the increase in stroke volume (SV) was 10\% or more, patients were considered hypovolemic and a new $250 \mathrm{~mL}$ bolus was given. This procedure was repeated until SV increase was less than $10 \%$. The maximal SV was maintained during surgery and corrected if necessary with 250 $\mathrm{mL}$ boluses Voluven. In the case of hypotension despite Doppler-guided volume therapy, vasoactive drugs were given as described above.

Postoperatively, patients were admitted to a standard post-anaesthetic care unit for at least six hours. The Doppler probe remained in situ for a maximum of six hours or until the patient experienced significant discomfort. In all patients, basic postoperative fluid management consisted of Ringer's solution $2-4 \mathrm{ml} / \mathrm{kg} / \mathrm{h}$. Furthermore, volemic status was assessed every hour by the passive leg raising test (PLR), which was performed with a standardized angle of $135^{\circ}$ between the trunk and lower limbs as described by Monnet et al. ${ }^{15}$. The optimal Doppler signal was obtained and stroke volume measurements were averaged over 5 beats. The 
same procedure was repeated after PLR. Doppler recordings were taken when the stroke volume reached its highest value (which was approximately 30 seconds after PLR). The maximum effect of PLR on stroke volume was seen within 1 minute in all patients. In the control group, PLR was performed but no additional fluid interventions were done. In the intervention group, if the increase in SV during PLR was $10 \%$ or more, patients were considered hypovolemic and a $250 \mathrm{~mL}$ bolus Voluven was given. In the control group, fluid boluses were administered based on standard hemodynamic and clinical parameters.

After discharge to a specialized colorectal surgery ward (at six hours postoperatively or more), patients were treated according to a multimodal fasttrack programme with early start of feeding and mobilization. Fluid therapy was equal in both groups: as soon as swallowing was considered safe, intake of fluids and nutrition was started, and a minimum fluid intake of 2 litres per day was aimed for. If this could not be reached by oral intake, i.v. fluid suppletion was given. Pain management was achieved by patient controlled analgesia via the epidural catheter or intravenously, for a maximum of three days postoperatively, and if needed additional paracetamol or morphine was given.

\section{Hemodynamic parameters}

The following hemodynamic parameters were monitored with 15-minute intervals during surgery and hourly during the first six hours after surgery: heart rate; stroke volume (Doppler); MAP (arterial line); and urinary output. Blood-soaked gauzes were weighted as they were passed off the surgical field and the blood content of the blood collection system was measured to assess total blood loss at the end of surgery.

\section{Blood sampling and processing}

Arterial blood samples were obtained from the radial artery line at predefined time points: baseline, every 30 minutes during surgery, and every hour until six hours postoperatively. Venous blood samples were taken daily until three days after surgery. Blood samples were collected in pre-chilled EDTA containing vacuum tubes (BD vacutainer, Becton Dickinson Diagnostics, Aalst, Belgium) and immediately centrifuged at $4{ }^{\circ} \mathrm{C}$ (2000 g, 15 minutes). Plasma samples were stored at $-80{ }^{\circ} \mathrm{C}$ until batch analysis. 


\section{Measurement of intestinal damage}

Intestinal fatty acid binding protein (I-FABP) plasma levels were determined using an in-house ELISA that selectively detects human I-FABP (lower detection limit: 25 $\mathrm{pg} / \mathrm{ml})$.

\section{Gastric tonometry}

A gastric tonometry catheter (14F, Medi-Line, Angleur, Belgium) was introduced transnasally for measurement of intramucosal carbon dioxide pressure $\left(\mathrm{P}_{\mathrm{r}} \mathrm{CO}_{2}\right.$ in $\mathrm{kPa}$ ) throughout the surgical procedure and during the six hours following surgery, using the gas-automated capnograph (Tonocap TC-200, Datex-Ohmeda, Helsinki, Finland). Gastric tonometry measurements $\left(\mathrm{P}_{\mathrm{r}} \mathrm{CO}_{2}\right.$, and mucosal-arterial $\mathrm{pCO}_{2}$ gap $\left(\mathrm{P}_{\mathrm{r}-\mathrm{a}} \mathrm{CO}_{2}\right.$-gap)) were done at 15-minute intervals during surgery and hourly during the first six hours following surgery. The first measurement during surgery was done at 15 minutes following the start of surgery, due to the calibration time of the device.

\section{Statistical analysis}

Statistical analysis was performed using Prism 5.0 for Windows (Graphpad software, Inc, San Diego, CA) and SPSS 20.0 for Windows (SPSS Inc, Chicago, IL). Normality was tested using Kolmogorov-Smirnov. The primary outcome was intestinal epithelial damage measured by plasma I-FABP levels at 1 hour postoperatively. Secondary outcomes were $\mathrm{P}_{\mathrm{r}-\mathrm{a}} \mathrm{CO}_{2}$-gap, and the hemodynamic parameters MAP, indexed stroke volume (SVI, stroke volume corrected for body surface area) and urinary production. For all repeated measures, areas under the curve (AUC) were calculated for each patient separately, and missing data were handled using multiple imputations in SPSS. Average AUCs of the outcomes were compared using Students $t$ test. Except for length of hospital stay (median and range), all continuous variables are presented as mean and standard deviation (SD). Dichotomous variables were compared using Chi-square test.

Sample size was calculated as follows. In a previous study, patients undergoing major non-abdominal surgery had mean I-FABP levels of 443 (SD, 309) pg/mL at the end of surgery ${ }^{16}$. In the current study, a reduction to 200 (SD, 150) pg/mL by hemodynamic optimization was estimated a priori, necessitating a sample size 
of 27 per group, with $\alpha=0.05$ and $1-\beta=0.95$. As a $5 \%$ drop-out rate was expected because of inability to achieve adequate Doppler measurements, the needed sample size was estimated at 29 for each group.

\section{Results}

\section{Patients}

Fifty-eight patients were randomized, of whom 27 were allocated to the intervention group (Figure 1). The study protocol was completed in all patients and none were excluded from analysis. Baseline characteristics are summarized

\section{CONSORT Flow Diagram}

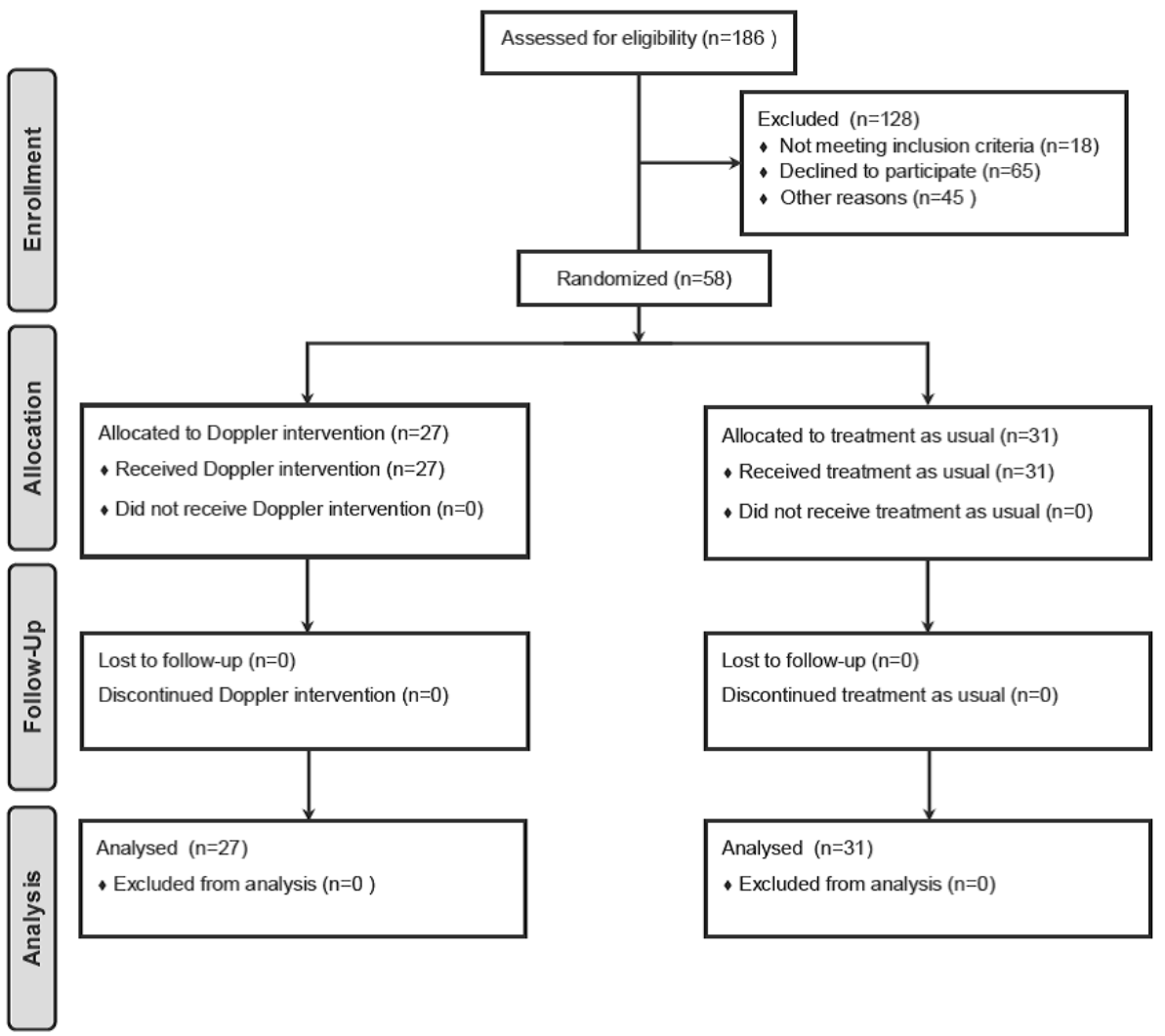

FIGURE 1. CONSORT diagram of the study. 
TABLE 1. Patient characteristics

\begin{tabular}{|c|c|c|c|c|c|}
\hline & & \multicolumn{2}{|c|}{ Intervention group } & \multicolumn{2}{|c|}{ Control group } \\
\hline & & Patients & Mean (SD) & Patients & Mean (SD) \\
\hline \multirow[t]{2}{*}{ Sex } & Male & $21(77.8 \%)$ & & $20(64.5 \%)$ & \\
\hline & Female & $6(22.2 \%)$ & & 11 (35.5\%) & \\
\hline \multirow[t]{2}{*}{ Age (years) } & & & $68.6(10.8)$ & & $67.6(10.0)$ \\
\hline & $>70$ & 10 (37.0\%) & & $12(38.7 \%)$ & \\
\hline \multirow[t]{2}{*}{ BMI $\left(\mathrm{kg} / \mathrm{m}^{2}\right)$} & & & 25.9 (3.1) & & $25.3(3.0)$ \\
\hline & $>25$ & 12 (44.4\%) & & 17 (54.8\%) & \\
\hline \multirow[t]{3}{*}{ ASA } & I & $8(29.6 \%)$ & & 4 (12.9\%) & \\
\hline & II & 14 (51.9\%) & & 24 (77.4\%) & \\
\hline & III & $5(18.5 \%)$ & & $3(9.7 \%)$ & \\
\hline \multirow{2}{*}{$\begin{array}{l}\text { Tumour } \\
\text { location }\end{array}$} & Colon & $11(40.7 \%)$ & & $13(41.9 \%)$ & \\
\hline & $\begin{array}{l}\text { Rectum/ } \\
\text { Sigmoid }\end{array}$ & $16(59.3 \%)$ & & 18 (58.1\%) & \\
\hline Smokers & & $6(22.2 \%)$ & & 5 (16.1\%) & \\
\hline \multirow[t]{4}{*}{$\begin{array}{l}\text { Medical } \\
\text { history }\end{array}$} & $\begin{array}{l}\text { Myocardial } \\
\text { ischemia }\end{array}$ & $1(3.7 \%)$ & & $1(3.2 \%)$ & \\
\hline & Stroke & $4(14.8 \%)$ & & $3(9.7 \%)$ & \\
\hline & NIDDM & 3 (11.1\%) & & $2(6.5 \%)$ & \\
\hline & COPD & $1(3.7 \%)$ & & $2(6.5 \%)$ & \\
\hline
\end{tabular}

BMI: body mass index; ASA: American Society of Anaesthesiologists; NIDDM: non-insulindependent diabetes mellitus; COPD: chronic obstructive pulmonary disease

TABLE 2. Surgery characteristics

\begin{tabular}{|c|c|c|c|c|c|}
\hline & & \multicolumn{2}{|c|}{ Intervention group } & \multicolumn{2}{|c|}{ Control group } \\
\hline & & Patients & Mean (SD) & Patients & Mean (SD) \\
\hline \multicolumn{2}{|c|}{ Surgical approach Open } & $20(74.1 \%)$ & & $21(67.7 \%)$ & \\
\hline & Laparoscopy & 7 (25.9\%) & & $10(32.3 \%)$ & \\
\hline \multirow[t]{5}{*}{ Type of surgery } & Right colectomy & $10(37.0 \%)$ & & $12(38.7 \%)$ & \\
\hline & Left colectomy & $1(3.7 \%)$ & & $0(0 \%)$ & \\
\hline & $\begin{array}{l}\text { Sigmoid } \\
\text { resection }\end{array}$ & 5 (18.5\%) & & $5(16.1 \%)$ & \\
\hline & Rectal resection & $11(40.7 \%)$ & & $13(41.9 \%)$ & \\
\hline & $\begin{array}{l}\text { Subtotal } \\
\text { colectomy }\end{array}$ & $0(0 \%)$ & & $1(3.2 \%)$ & \\
\hline Epidural & & $21(77.8 \%)$ & & 22 (71.0\%) & \\
\hline Ostomy & & $12(44.4 \%)$ & & $14(45.2 \%)$ & \\
\hline $\begin{array}{l}\text { Operative time } \\
\text { (minutes) }\end{array}$ & & & $256(101)$ & & $205(77)$ \\
\hline
\end{tabular}


in Table 1. No differences were observed between the intervention and control groups. Stratification for open and laparoscopic surgery was verified, and other operative characteristics are outlined in Table 2. Operative time was higher in the intervention group. More colloid fluid was given in the intervention group (Table 3), while total administered fluids did not differ between the groups. The mean time the Doppler probe was tolerated was 4 hours postoperatively.

TABLE 3. Fluids during surgery

\begin{tabular}{|c|c|c|c|c|c|}
\hline & & \multicolumn{2}{|c|}{ Intervention group } & \multicolumn{2}{|c|}{ Control group } \\
\hline & & Patients & Mean (SD) & Patients & Mean (SD) \\
\hline \multirow[t]{2}{*}{ Total amount (mL) } & Crystalloids & & $\begin{array}{l}3,000 \\
(1,093)\end{array}$ & & $\begin{array}{l}3,026 \\
(1,307)\end{array}$ \\
\hline & Colloids & & $1,526(823)$ & & $952(687)$ \\
\hline Total fluid (mL/kg/h) & & & $14.6(4.7)$ & & 16.2 (5.9) \\
\hline Blood loss (mL) & & & $957(1,880)$ & & $461(1,026)$ \\
\hline & $>750 \mathrm{~mL}$ & $6(22.2 \%)$ & & $4(12.9 \%)$ & \\
\hline Blood transfusion & & 7 (25.9\%) & & $4(12.9 \%)$ & \\
\hline Total fluid $(\mathrm{mL} / \mathrm{kg} / \mathrm{h})^{1}$ & & & $15.8(6.4)$ & & $16.7(6.5)$ \\
\hline Vasopressor use & & 12 (44.4\%) & & $15(48.4 \%)$ & \\
\hline
\end{tabular}

${ }^{1}$ Including blood transfusions

\section{Hemodynamic changes}

A significant increase in SVI from baseline to start of surgery was accomplished by colloid administration in the group receiving hemodynamic optimization (46.5 (12.0) $\mathrm{mL} / \mathrm{m}^{2}$ to $59.8(15.7) \mathrm{mL} / \mathrm{m}^{2}, p<0.0001$, Figure $\left.2 A\right)$. Of 27 patients in the intervention group, 24 (89\%) needed fluid to establish a maximal stroke volume. A significant SVI increase from baseline to start of surgery was not observed in the control group. Furthermore, mean AUC of SVI during surgery were higher in the intervention group $\left(12,631(2,568) \mathrm{mL} / \mathrm{m}^{2} \times\right.$ min) compared with the control group $\left(11,122(2,014) \mathrm{mL} / \mathrm{m}^{2} \times \mathrm{min}, p=0.02\right)$. Mean AUC of postoperative SVI were not significantly different between groups (Figure $2 \mathrm{~B}$ ).

MAP (Figure 3) and urinary output (Figure 4) were not significantly different between the intervention and control groups. 

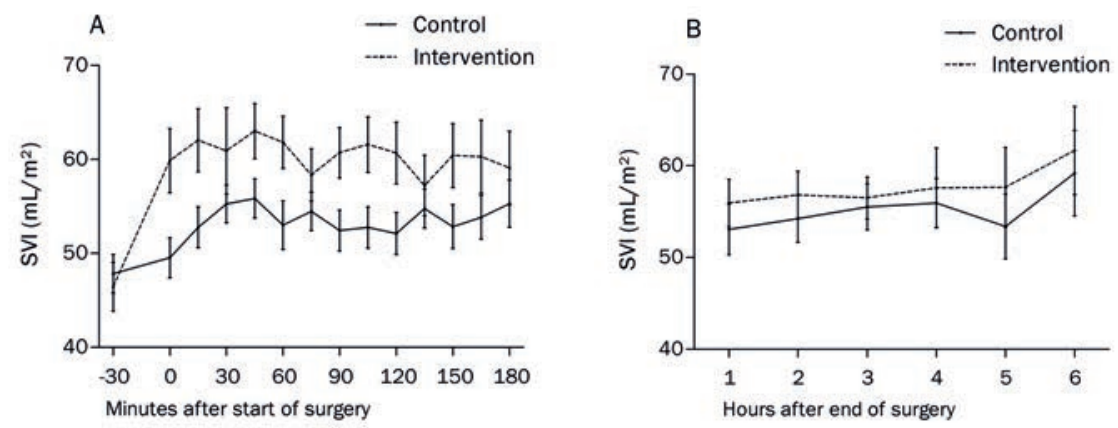

FIGURE 2. Mean indexed stroke volumes (SVI, corrected for body surface area) during surgery (A) and during the first six hours after surgery (B). Error bars are SEM (standard error of the mean).
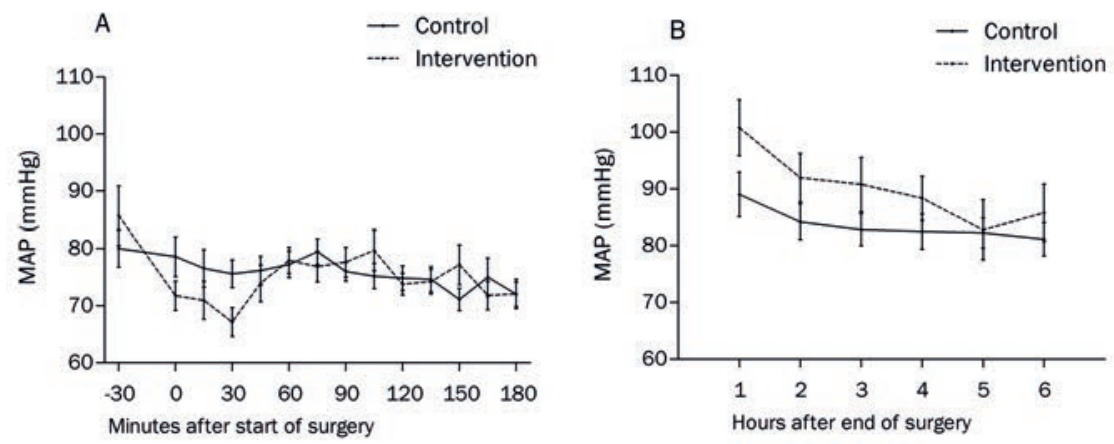

FIGURE 3. Mean arterial pressures (MAP) during surgery $(A)$ and during the first six hours after surgery (B). Error bars are SEM.
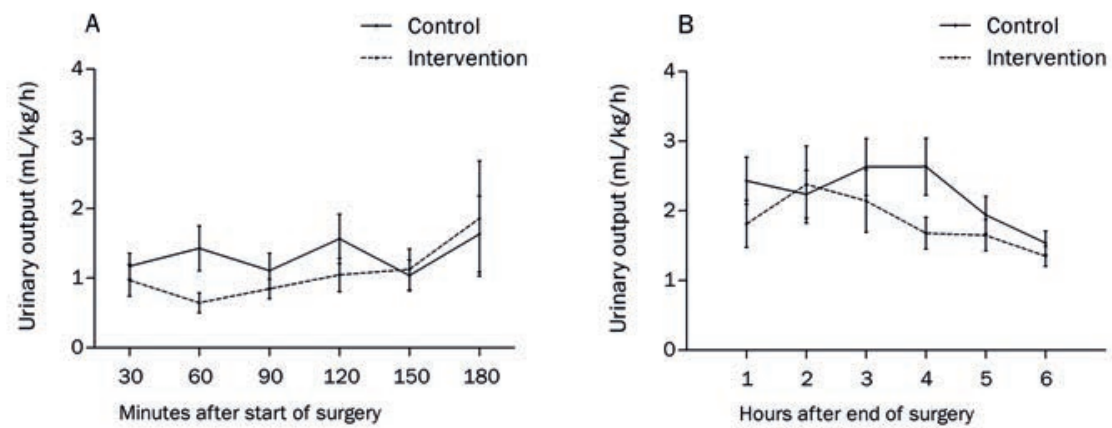

FIGURE 4. Mean urinary output per hour corrected for body weight during surgery $(A)$ and during the first six hours after surgery (B). Errors bars are SEM. 


\section{Intestinal damage}

Mean I-FABP levels 1 hour after surgery were 488.6 (599.2) pg/mL in the total cohort. No significant differences of mean I-FABP levels in the intervention group compared with the control group were observed at this time-point (respectively, $440.8(251.6) \mathrm{pg} / \mathrm{mL}$ and 522.4 (759.9) pg/mL, $p=0.67$ ), nor at another time-point (Figure 5). In addition, I-FABP levels did not increase during surgery both groups, indicating the absence of significant intestinal damage. I-FABP levels significantly decreased in both groups from start of surgery to the first day postoperatively (respectively from 600.5 (826.0) to 384.2 (550.3), mean of all patients, $p=0.03$ ). Mean AUC of the IFABP concentration was not significantly different between the groups.

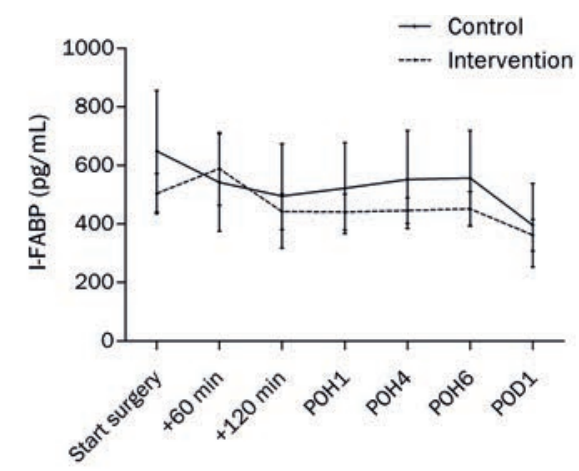

FIGURE 5. Intestinal Fatty Acid Binding Protein (I-FABP) levels during and after surgery. +60 min: 1 hour after start of surgery; +120 min: 2 hours after start of surgery; POHx: x hour(s) postoperatively.

\section{Gastric tonometry}

Intraoperative arterial $\mathrm{CO}_{2}$ pressures were not different between the intervention group (5.0 (0.4) kPa) and the control group (4.9 (0.3) $\mathrm{kPa}$ ). Mean AUC of the $\mathrm{P}_{\mathrm{r}}$ ${ }_{a} \mathrm{CO}_{2}$-gap during surgery was significantly lower in the intervention group (4.8 (98.9) kPa x min) compared to the control group (91.7 (92.0) kPa x min, $p=0.01$ ), indicating better global gastrointestinal perfusion in the intervention group (Figure $6 \mathrm{~A})$. The mean intraoperative $\mathrm{P}_{\mathrm{r}-\mathrm{a}} \mathrm{CO}_{2}$-gap peak in the intervention group was 0.5 (1.0) $\mathrm{kPa}$, which was significantly lower than the peak in the control group; 1.4 (1.4) $\mathrm{kPa}, p=0.03$. However, no effect of stroke volume optimization on gastrointestinal perfusion was observed in the first six hours after surgery in terms of $\mathrm{P}_{\mathrm{r}-\mathrm{a}} \mathrm{CO}_{2}$-gap AUCs or peak (Figure 6B). 

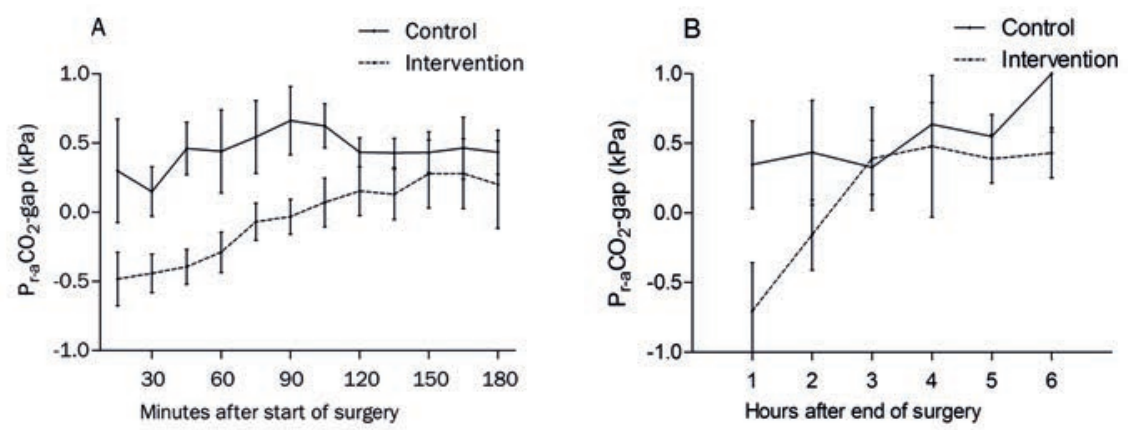

FIGURE 6. Pr-aCO2-gap during surgery (A), and during the first six hours after surgery (B). Error bars are SEM.

Postoperative arterial $\mathrm{CO}_{2}$ pressures were not different between the intervention group (5.6 (0.9) kPa) and the control group (5.4 (0.6) kPa). Mean AUC of the postoperative $\mathrm{P}_{\mathrm{r}-\mathrm{a}} \mathrm{CO}_{2}$-gap was elevated in patients with major blood loss during surgery (>750 mL, $\mathrm{n}=10$ ); 6.7 (8.3) $\mathrm{kPa} \times \mathrm{h}$ compared to 0.6 (3.3) kPa in patients without major blood loss ( $p=0.005$, data not shown). In linear regression analysis, major blood loss was a significant independent predictor for increasing $\mathrm{P}_{\mathrm{r}-\mathrm{a}} \mathrm{CO}_{2}$ gap postoperatively ( $\beta=0.55, p=0.001$ ), and group (intervention) showed a trend towards significance $(\beta=-0.27, p=0.07)$.

When only patients without major blood loss were analysed, mean postoperative AUC of the $\mathrm{P}_{\mathrm{r}-\mathrm{a}} \mathrm{CO}_{2}$-gap was lower in the intervention group (-0.7 (3.9) $\mathrm{kPa} \times \mathrm{h}$ compared to 2.0 (2.3) $\mathrm{kPa} \times \mathrm{h}$ in the control group, $p=0.04)$. Furthermore, the mean postoperative $\mathrm{P}_{\mathrm{r}-\mathrm{a}} \mathrm{CO}_{2}$-gap peak in the intervention group was $0.6(0.7) \mathrm{kPa}$ in this subgroup, significantly lower than the peak in the control group; 1.4 (1.2) $\mathrm{kPa}, p=0.04$.

\section{Clinical outcome}

Postoperative mortality, complications and length of hospital stay are summarized in Table 4. No significant differences in mortality or complications were found between the control and intervention groups. Length of hospital stay was significantly increased in the intervention group compared with the control group (median 11 (range, $4-50$ ), and 8 (5 - 26), respectively, $p=0.03$ ). 
TABLE 4. Clinical outcome

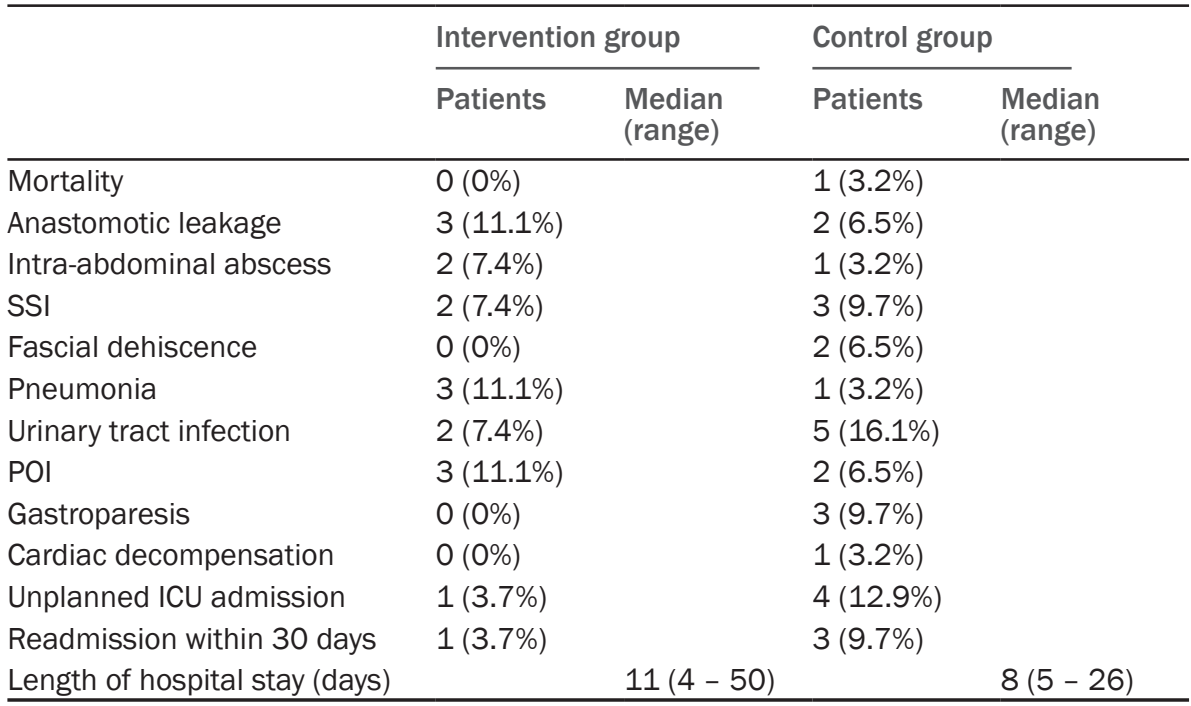

SSI: surgical site infection; POI: postoperative ileus; ICU: intensive care unit

\section{Discussion}

This randomized controlled trial showed that Doppler guided GDFT during and after elective colorectal surgery for malignancy increases global gastrointestinal perfusion. However, no significant differences in plasma I-FABP levels between the intervention and control group were observed. In addition, both groups showed no significant intestinal damage. A strong positive effect of Doppler guided GDFT on gastrointestinal perfusion was seen during surgery. GDFT showed a marginally significant effect on postoperative gastrointestinal perfusion, and only when corrected for major intraoperative blood loss (>750 mL). It was hypothesized that plasma I-FABP levels would peak compared to baseline at about one hour after the end of surgery as was observed before in non-abdominal surgery with ${ }^{17,18}$ and without ${ }^{16}$ aortic cross-clamping. The latter study investigating scoliosis repair in children showed that low MAPs are associated with increased I-FABP levels. In this study, average MAPs during surgery were $64 \mathrm{mmHg}$ compared to $75 \mathrm{mmHg}$ in the current study. It may therefore be speculated that patients undergoing elective colorectal surgery do not exhibit severe enough hypotension to develop intestinal damage. Nonetheless, increased gastrointestinal perfusion due to GDFT indicates 
a rather euvolemic status in these patients, since the gut is one of the organs that are primarily affected by the redistribution of blood to the vital organs in (beginning) hypovolemia ${ }^{19}$.

The discrepancy between the effect of GDFT on intraoperative in contrast to postoperative perfusion may be explained by the methodological differences of fluid optimization during and after surgery. This was underlined by higher mean SVI in the intervention group compared to the control group intraoperatively, but not postoperatively. Just before and during surgery, the stroke volume was optimized by fluid challenges, while after surgery, fluid responsiveness was assessed by PLR and fluid challenges were only given when the PLR was positive. Although PLR represents good sensitivity $(77 \%)$ to detect fluid responsiveness, some patients that should receive a fluid bolus (23\%) are inevitably missed ${ }^{20}$. Moreover, other factors may be more important predictors of postoperative gastrointestinal perfusion, as was indicated by the strong association of major intraoperative blood loss and decreased postoperative gastrointestinal perfusion.

Interestingly, the amount of fluid given did not differ between the intervention and the control group, indicating that rather timing of fluid administration was the determinant of improving global and regional hemodynamics, thereby shedding new light on the concept of euvolemia. This observation is in line with previous work describing more stable hemodynamic parameters when stroke volumebased optimization was applied even though total amount of fluid given was comparable with the control group ${ }^{21}$. Another interesting finding was the need for fluid expansion in $89 \%$ of patients to establish maximal stroke volumes, which is in line with a previous study showing a functional volume deficit in $70 \%$ of patients undergoing different types of surgery ${ }^{22}$. This underlines the possible benefits of patient-tailored GDFT. However, it remains unclear whether such deficits represent actual susceptibility to complications or rather increased physiological reserves.

Several other randomized clinical trials have been performed on Doppler guided GDFT in major bowel surgery 2-6, 13, 14. Although some trials showed a significantly shortened length of hospital stay, decreased morbidity and increased gut function ${ }^{3,4}$, the largest ${ }^{6,13}$ and most recent ${ }^{14}$ trials showed no advantage of Doppler guided GDFT over standard or restrictive fluid therapy. Challand and coworkers even showed that GDFT increases length of hospital stay in aerobically fit patients ${ }^{6}$. This supports the hypothesis that sub-maximal stroke volumes reflect physiological reserves instead of a pathological deficit, and stroke volume optimization, although improving gut perfusion, can actually lead to fluid overload 
in these patients. The present study adds the application of Doppler guided GDFT in the early postoperative phase. However, no or only marginally significant effects of the intervention were seen postoperatively in terms of gastrointestinal perfusion or SVI. Therefore, GDFT has no additional effect compared with standard fluid treatment in the postoperative phase based on the current results.

The present study was not designed to detect differences in clinical outcome. Although we show that stroke volume optimization improves gastrointestinal perfusion, it remains to be determined in which patients this approach leads to better clinical outcome. As noted in the Challand trial ${ }^{6}$, patients that have low oxygen consumption levels may benefit from GDFT, however large numbers of such a selected population are needed and not easily obtainable. Moreover, caution should be taken when interpreting the gut tonometry data, for the measurements were done in the stomach as a reflection of overall gastrointestinal perfusion. In colorectal surgery, an important target of fluid therapy is to establish adequate perfusion of the gut and the anastomosis in particular, to diminish the risk of postoperative complications such as anastomotic leakage and gut-derived sepsis. It is not known whether this generalized read-out accurately correlates with local (hypo)perfusion. Other techniques, such as in vivo microscopy ${ }^{23}$ could be accurate tools for detecting low local perfusion.

In conclusion, Doppler guided GDFT increased global gastrointestinal perfusion in this study in patients undergoing elective colorectal surgery, indicating a rather euvolemic state in these patients. This provides new evidence for the implementation of GDFT in clinical practice. However, the definition of clinically important euvolemia deserves further exploration, since the clinical benefit of GDFT over other strategies was not unequivocally proven in previous trials. 


\section{References}

1. Rahbari NN, Zimmermann JB, Schmidt T, et al. Meta-analysis of standard, restrictive and supplemental fluid administration in colorectal surgery. Br J Surg 2009;96:33141.

2. Conway $\mathrm{DH}$, Mayall R, Abdul-Latif MS, et al. Randomised controlled trial investigating the influence of intravenous fluid titration using oesophageal Doppler monitoring during bowel surgery. Anaesthesia 2002;57:845-9.

3. Noblett SE, Snowden CP, Shenton BK, et al. Randomized clinical trial assessing the effect of Doppler-optimized fluid management on outcome after elective colorectal resection. Br J Surg 2006;93:1069-76.

4. Wakeling HG, McFall MR, Jenkins CS, et al. Intraoperative oesophageal Doppler guided fluid management shortens postoperative hospital stay after major bowel surgery. Br J Anaesth 2005;95:634-42.

5. Senagore AJ, Emery T, Luchtefeld M, et al. Fluid management for laparoscopic colectomy: a prospective, randomized assessment of goal-directed administration of balanced salt solution or hetastarch coupled with an enhanced recovery program. Dis Colon Rectum 2009;52:1935-40.

6. Challand C, Struthers R, Sneyd JR, et al. Randomized controlled trial of intraoperative goal-directed fluid therapy in aerobically fit and unfit patients having major colorectal surgery. Br J Anaesth 2012;108:53-62.

7. Klotz KF, Klingsiek S, Singer M, et al. Continuous measurement of cardiac output during aortic cross-clamping by the oesophageal Doppler monitor ODM 1. Br J Anaesth 1995;74:655-60.

8. Donati A, Munch C, Marini B, et al. Transesophageal Doppler ultrasonography evaluation of hemodynamic changes during videolaparoscopic cholecystectomy. Minerva Anestesiol 2002;68:549-54.

9. Roy N, Maw A, Stuart-Smith K. Fluid optimization guided by oesophageal Doppler significantly improves bowel perfusion. Br J Anaesth 2011;107:1012-3.

10. Holte K, Sharrock NE, Kehlet H. Pathophysiology and clinical implications of perioperative fluid excess. Br J Anaesth 2002;89:622-32.

11. Poeze M, Ramsay G, Greve JW, et al. Prediction of postoperative cardiac surgical morbidity and organ failure within 4 hours of intensive care unit admission using esophageal Doppler ultrasonography. Crit Care Med 1999;27:1288-94.

12. Brandstrup B. Fluid therapy for the surgical patient. Best Pract Res Clin Anaesthesiol 2006;20:265-83.

13. Brandstrup B, Svendsen PE, Rasmussen M, et al. Which goal for fluid therapy during colorectal surgery is followed by the best outcome: near-maximal stroke volume or zero fluid balance? Br J Anaesth 2012;109:191-9.

14. Srinivasa S, Taylor MH, Singh PP, et al. Randomized clinical trial of goal-directed fluid therapy within an enhanced recovery protocol for elective colectomy. Br J Surg 2013;100:66-74. 
15. Monnet X, Rienzo M, Osman D, et al. Passive leg raising predicts fluid responsiveness in the critically ill. Crit Care Med 2006;34:1402-7.

16. Derikx JP, van Waardenburg DA, Thuijls G, et al. New Insight in Loss of Gut Barrier during Major Non-Abdominal Surgery. PLoS One 2008;3:e3954.

17. Vermeulen Windsant IC, Hellenthal FA, Derikx JP, et al. Circulating intestinal fatty acid-binding protein as an early marker of intestinal necrosis after aortic surgery: a prospective observational cohort study. Ann Surg 2012;255:796-803.

18. Hanssen SJ, Derikx JP, Vermeulen Windsant IC, et al. Visceral injury and systemic inflammation in patients undergoing extracorporeal circulation during aortic surgery. Ann Surg 2008;248:117-25.

19. Moore FA. The role of the gastrointestinal tract in postinjury multiple organ failure. Am J Surg 1999;178:449-53.

20. Lamia B, Ochagavia A, Monnet X, et al. Echocardiographic prediction of volume responsiveness in critically ill patients with spontaneously breathing activity. Intensive Care Med 2007;33:1125-32.

21. Rinehart J, Chung E, Canales C, et al. Intraoperative stroke volume optimization using stroke volume, arterial pressure, and heart rate: closed-loop (learning intravenous resuscitator) versus anesthesiologists. J Cardiothorac Vasc Anesth 2012;26:933-9.

22. Bundgaard-Nielsen $\mathrm{M}$, Jorgensen $\mathrm{CC}$, Secher $\mathrm{NH}$, et al. Functional intravascular volume deficit in patients before surgery. Acta Anaesthesiol Scand 2010;54:464-9.

23. Goedhart PT, Khalilzada M, Bezemer R, et al. Sidestream Dark Field (SDF) imaging: a novel stroboscopic LED ring-based imaging modality for clinical assessment of the microcirculation. Opt Express 2007;15:15101-14. 


Chapter 3. Cyclooxygenase-2 is essential for colorectal anastomotic healing

Kostan W. Reisinger, Dirk H.S.M. Schellekens, Joanna W.A.M. Bosmans, Bas Boonen, Maarten F. von Meyenfeldt, Prapto Sastrowijoto, Joep P.M. Derikx, Joep Grootjans, Martijn Poeze. Submitted 


\begin{abstract}
Background

Cyclooxygenase-2 (COX-2) is a key enzyme in gastrointestinal homeostasis, affecting angiogenesis, profileration, inflammation and apoptosis. In this study the effects of COX-2 in colonic surgical wound healing were investigated. We provide evidence that COX-2 is essential for neovascularization of the colonic anastomosis and thereby plays a crucial role in colonic anastomotic wound healing.
\end{abstract}

\title{
Methods
}

Mice of different COX-2 genotypes were subjected to a model of colonic anastomotic leakage, and received vehicle, diclofenac or prostaglandin $E_{2}\left(P_{E_{2}}\right)$. Endpoints for anastomotic healing were incidence of anastomotic leakage and mortality. Angiogenesis was assessed in anastomotic tissue.

\section{Results}

All mice receiving diclofenac developed anastomotic leakage. Of mice completely lacking COX-2, 92\% developed anastomotic leakage, compared to $25 \%$ of wildtypes $(p=0.003)$. Supplementation with $\mathrm{PGE}_{2}$ decreased the anastomotic leakage rate in $\mathrm{COX}-2^{-1-}$ mice from $92 \%$ to only $46 \%(p=0.02)$. Quantification of the amount of blood vessels showed that only 2 vessels $/ \mathrm{mm}^{2}$ were stained in anastomotic tissue of COX-2 $-1-$ mice compared to 6 vessels $/ \mathrm{mm}^{2}$ in wildtype mice $(p=0.03)$. This effect could partly be reversed by administration of $\mathrm{PGE}_{2}$ to $\mathrm{COX}-2^{-1}$ mice.

\section{Conclusion}

COX-2 functioning is essential for intestinal wound healing after colonic surgery, which is mediated by $\mathrm{PGE}_{2}$ production. In addition, angiogenesis is significantly impaired in the absence of COX-2 and $\mathrm{PGE}_{2}$. Future research should aim at improving $\mathrm{COX}-2$ and $\mathrm{PGE}_{2}$ functioning in situations when adequate intestinal wound healing is of high importance. 


\section{Introduction}

The enzyme cyclooxygenase-2 (COX-2, also known as prostaglandin-endoperoxide syntase 2 (Ptgs2)) plays an important role in preserving gut homeostasis. In the colon, COX-2 is expressed constitutively in mesenchymal stem cells, producing prostaglandins which is believed to have an immunomodulatory role ${ }^{1}$. These mesenchymal stem cells may act as monitors of the colonic environment and are important in colonic wound healing ${ }^{2}$. In addition, COX-2 expression can be induced in macrophages and myofibroblasts upon exposure to proinflammatory cytokines and bacterial products ${ }^{1,3,4}$. Cyclooxygenase regulates the conversion of arachidonic acid into prostaglandins, of which $\mathrm{PGE}_{2}$ is reported to restore intestinal integrity in experimental models of intestinal inflammation and damage 1, 2, 4.6. Furthermore, COX-2 and $\mathrm{PGE}_{2}$ are critically involved in vascular endothelial growth factor (VEGF)-induced angiogenesis ${ }^{7,8}$.

Data from human studies underline the importance of COX-2 in colonic wound healing as the use of NSAIDs, especially those with strong COX-2-inhibiting properties, is markedly correlated with anastomotic leakage, i.e. inadequate wound healing following colorectal surgery ${ }^{9-12}$. These are however retrospective studies and a recent meta-analysis could not prove an unambiguous detrimental effect of NSAIDs ${ }^{13}$. These studies emphasize the importance of precautious NSAID use in patients with intestinal anastomoses, however omission of NSAIDs from postsurgical care in these patients is not yet standard clinical practice ${ }^{14}$, 15. Studies showing the mechanisms by which NSAIDs exert their effects on colonic surgical wound healing are essential to ban the use of NSAIDs for pain management after colonic surgery.

In this study the effects of COX-2 and $\mathrm{PGE}_{2}$ in colonic surgical wound healing were investigated. We provide evidence that COX-2 is essential for neovascularization of the colonic anastomosis and thereby plays a crucial role in colonic anastomotic wound healing. In line, blocking COX-2 either by using COX$2 \%$ mice or by administration of NSAIDs was not only associated with significantly higher anastomic leakage rates but also with increased mortality. These data further emphasize that COX-2 inhibitors should be avoided in patients undergoing colonic surgery. 


\section{Materials and methods}

Mice

All animal experiments were approved by the Maastricht University Animal Experiments Committee. COX-2 $2^{+/-}$mice were obtained from the Jackson Laboratory (Bar Harbor, ME) to produce littermate wildtype (WT), COX-2 ${ }^{+/-}$and COX-2 $2^{--}$mice. For all experiments, COX-2\%- mice were used simultaneously with their wildtype and/or heterozygous littermates, resulting in various group sizes.

\section{Model}

A previously described murine model of colonic anastomotic leakage was used and adapted to obtain an anastomotic leakage rate of $25-33 \%$, to obtain a model that resembles clinical practice ${ }^{16}$. Briefly, 10- to 12 -week-old mice were anesthetized using isoflurane and buprenorphine. After a $1 \mathrm{~cm}$ midline laparotomy, the cecum was exteriorized and the right colon was microscopically transected, without damaging blood supply. An end-to-end anastomosis was performed with 7 interrupted sutures (Prolene 8-0, Ethicon, Somerville, NJ). The colon was repositioned and the abdomen was closed in two layers of interrupted sutures (Vicryl 4-0, Ethicon, and Ethilon 4-0, Ethicon, respectively). The colon was kept moist with sterile $\mathrm{NaCl}$ during the procedure. The surgical procedure was performed by two medically trained researchers that had equal skill and experience with the surgical procedure.

\section{Study design}

WT, COX $-2 \%$ and COX-2\% mice underwent laparotomy with colonic anastomosis, and received vehicle (PBS), 16,16-dimethyl $\mathrm{PGE}_{2}\left(\mathrm{dmPGE}_{2}\right.$, Cayman Chemical, Ann Arbor, $\mathrm{MI}$ ), a stable analogue of $\mathrm{PGE}_{2}$, or diclofenac sodium (Cayman Chemical) by intraperitoneal injection. Dosage of $\mathrm{dmPGE}_{2}$ was $100 \mu \mathrm{g} / \mathrm{kg}$ bodyweight, twice daily, and dosage of diclofenac was $10 \mathrm{mg} / \mathrm{kg}$ bodyweight, twice daily. Vehicle, $\mathrm{dmPGE}_{2}$ and diclofenac treatment was started one day prior to laparotomy and continued until the end of the experiments. Mice were sacrificed at 5 days postoperatively to ensure detection of anastomotic leakage, or when humane endpoints were reached. 


\section{Endpoints}

Anastomotic leakage was defined as one of the following: fecal peritonitis or abscess formation around the anastomosis. Anastomotic leakage was diagnosed by two independent investigators who were blinded for genotypes.

\section{Tissue preparation}

In anesthetized mice, a $1 \mathrm{~cm}$ segment of colonic tissue surrounding the anastomosis was dissected in longitudinal direction, thereby dividing it into equal parts, and fixed in formalin for immunohistochemistry purposes, or snap frozen in liquid nitrogen for qPCR.

\section{Immunohistochemistry}

For immunohistochemistry, sections were deparaffinized in xylene and rehydrated in graded ethanol to distilled water. Endogenous peroxidase activity was blocked using $0.6 \%$ hydrogen peroxide in methanol for $30 \mathrm{~min}$. Non-specific antibody binding was blocked using $5 \%$ bovine serum albumin (BSA) and sections were incubated with primary antibody rabbit anti-human myeloperoxidase (MPO, DakoCytomation, Glostrup, Denmark), which cross-reacts with mouse). Biotinconjugated swine anti-rabbit IgG (DakoCytomation) was used followed by incubation with the streptavidin-biotin-HRP system (DakoCytomation). Binding of primary antibody was visualized with 3,3'-diaminobenzidine-tetrahydrochloridedihydrate (Sigma, St Louis, MO) and counterstained with haematoxylin. No staining was detected in slides incubated without primary antibody.

For CD31 staining, an enzymatic antigen retrieval step was used prior to the nonspecific antibody blocking step using 0.1\% trypsin (Difco Laboratories, Detroit, MI) in $0.1 \% \mathrm{CaCl}_{2}$ solution for 20 minutes at $37^{\circ} \mathrm{C}$. Sections were incubated overnight at $4^{\circ} \mathrm{C}$ with rat anti-mouse CD31 primary antibody (BD Pharmingen, Breda, the Netherlands). After washing, biotinylated rabbit anti-rat IgG (DakoCytomation) was used as secondary antibody, followed by incubation with Brightvision poly HRP antirabbit IgG (Immunologic, Duiven, the Netherlands). Binding of primary antibodies was visualized with 3,3'-diaminobenzidinetetrahydrochloride-dihydrate (Sigma). Sections were counterstained with haematoxylin. No staining was detected in slides incubated without primary antibody. The CD31 staining was quantified in 
a blinded way by two observers by counting all CD31 positive vessels at 200x magnification and expressed as the number of vessels per total area $\left(\mathrm{mm}^{2}\right)$ using ImageJ (NIH Software, Bethesda, MD).

qPCR

RNA was isolated from snap-frozen anastomotic tissue samples with AllPrep DNA/RNA/Protein kit (Qiagen, Hilden, Germany) according to the manufacturer's protocol. In short, samples were crushed with a pestle and mortar in liquid nitrogen. Disruption and homogenisation of the tissue was performed using an Ultra Turrax Homogeniser (IKA Labortechnik, Staufen, Germany) in lysis buffer containing $\beta$-mercaptoethanol (Promega, Madison, WI). RNeasy spin columns were used to bind RNA. Columns were washed and RNA was eluted in RNase-free water. To analyse gene expression, qPCR was performed. All samples were treated with RNAse (Promega) to ensure complete removal of genomic DNA. Quantity was measured using the NanoDrop spectrophotometer (Thermo Scientific, Wilmington, DE). Only RNA samples with a clearly visible S28 and S18 on agarose gel were considered as intact RNA and were used. Total cDNA was synthesized using the iScript cDNA synthesis kit (Bio-Rad, Hercules, CA). qPCR reactions were performed on $10 \mathrm{ng}$ cDNA with $300 \mathrm{nM}$ of gene-specific forward and reverse primers and $1 \times$ Absolute qPCR SYBR Green Fluorescein Mix (Bioline, London, United Kingdom) using the MylQ system (Bio-Rad). Primers used were m-VegfA-f1 (TATTCAGCGGACTCACCAGC); and m-VegfA-r1 (CCTCCTCAAACCGTTGGCA). Gene expression levels were calculated with IQ5 software using a $\triangle \mathrm{Ct}$ relative quantification model. The geometric mean of two internal control genes ( $\beta 2$ microglobulin and cyclophilin A) was calculated and used as a normalization factor.

\section{Statistics}

Statistical analysis was performed using Prism 5.0 for Windows (Graphpad software, Inc, San Diego, CA) and SPSS 20.0 for Windows (SPSS Inc, Chicago, IL). Normality was tested using Kolmogorov-Smirnov. All continuous variables are presented as mean and standard error of the mean (SEM) and compared using students $t$-test. Dichotomous variables were compared using Chi-square test. Survival was analyzed by logrank test. 


\section{Results}

Diclofenac is associated with high rates of colonic anastomotic leakage in an experimental model

To study whether the observed retrospective human data on colonic anastomic leakage in patients receiving COX-2 inhibitors were also observed in mice, anastomotic leakage rates in mice receiving either vehicle or NSAIDs were determined. Three out of eleven mice in the wildtype group developed anastomotic leakage (27\%). Intriguingly, all 9 mice receiving diclofenac (100\%) developed anastomotic leakage, $p=0.001$ (Figure 1A). In line, survival was significantly reduced in the group receiving diclofenac compared to the group receiving vehicle (hazard ratio (HR), 17.9 (95\% confidence interval (Cl), 3.7 - 87.4), $p<0.001$, Figure 1B). These data confirm the detrimental effects of NSAIDs on anastomotic healing.
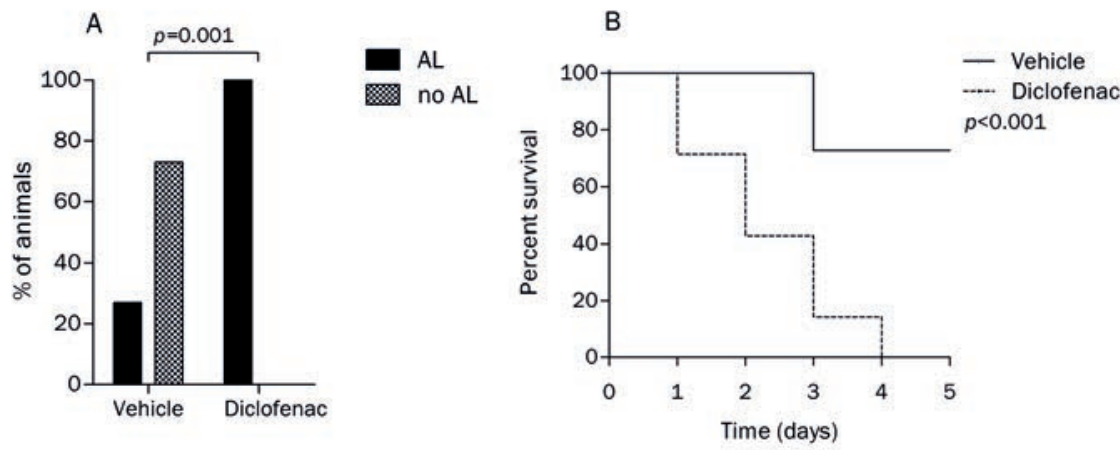

FIGURE 1. Effect of diclofenac on experimental anastomotic healing. A. Incidence of anastomotic leakage in mice treated with vehicle $(n=11)$ and mice treated with diclofenac $(n=9)$. B. Survival of mice treated with vehicle and mice treated with diclofenac. Mice treated with diclofenac showed higher anastomotic leakage incidence and mortality rates.

\section{Cyclooxygenase 2 is critically involved in colonic anastomotic healing}

It was hypothesized that the adverse effects of NSAIDs were particularly a consequence of blocking COX-2. We therefore investigated the selective effects of COX-2 in anastomotic healing in COX-2\% mice. Eleven out of twelve COX-2\% mice had macroscopic leakage (92\%) compared to only $27 \%$ of WT mice ( $p=0.003$, Figure 2A). Survival was significantly reduced in COX-2 $\%$ mice compared to WT mice (HR, $3.5(95 \% \mathrm{Cl}, 1.2-10.3), p=0.02$, Figure $2 \mathrm{~B})$. 

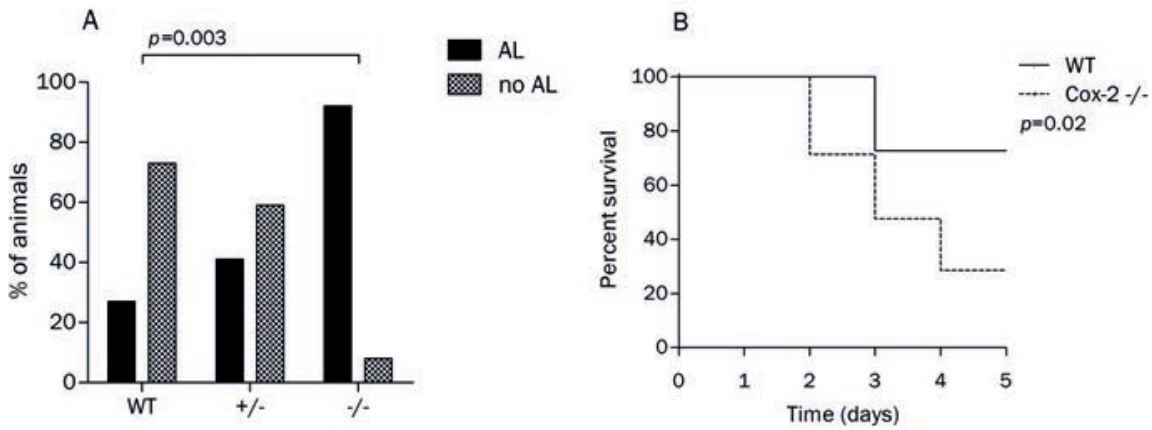

FIGURE 2. Effect of genotype on experimental anastomotic healing. A. Incidence of anastomotic leakage in wildtype $(n=11)$, COX-2+/- $(n=17)$, and COX-2-/- $(n=12)$ mice. B. Survival of wildtype and COX-2-/- mice. COX-2-/- mice showed higher anastomotic leakage and mortality rates compared to wildtype mice.

Since COX-2 is essential for the production of $\mathrm{PGE}_{2}$, which is an important mediator in gut homeostasis, we investigated whether $\mathrm{PGE}_{2}$ administration could rescue $\mathrm{COX}_{-2} \%$ from anastomotic leakage. Intriguingly, supplementation with $\mathrm{dmPGE}_{2}$ decreased the anastomotic leakage rate in COX-2/- mice from 92\% (11 out of 12 mice) to only $46 \%$ (6/13 mice), $p=0.02$ (Figure 3 ).

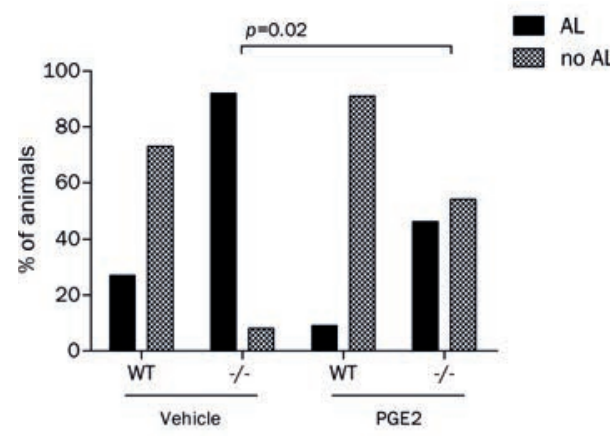

FIGURE 3. Effect of PGE2 administration on anastomotic leakage rates in wildtype $(n=12)$ and COX-2/- $(n=13)$ mice. PGE2 administration decreased anastomotic leakage rates in COX-2-/- mice.

\section{Lack of COX-2 does not lead to inflammatory changes in anastomotic tissue}

Since COX-2 is involved in a wide variety of (patho)physiological processes, our next goal was to dig further into the mechanisms involved in COX-2 dependent impaired anastomotic healing. One of the obvious mechanisms that could be disturbed by blocking COX-2 function is the inflammatory response, which is 

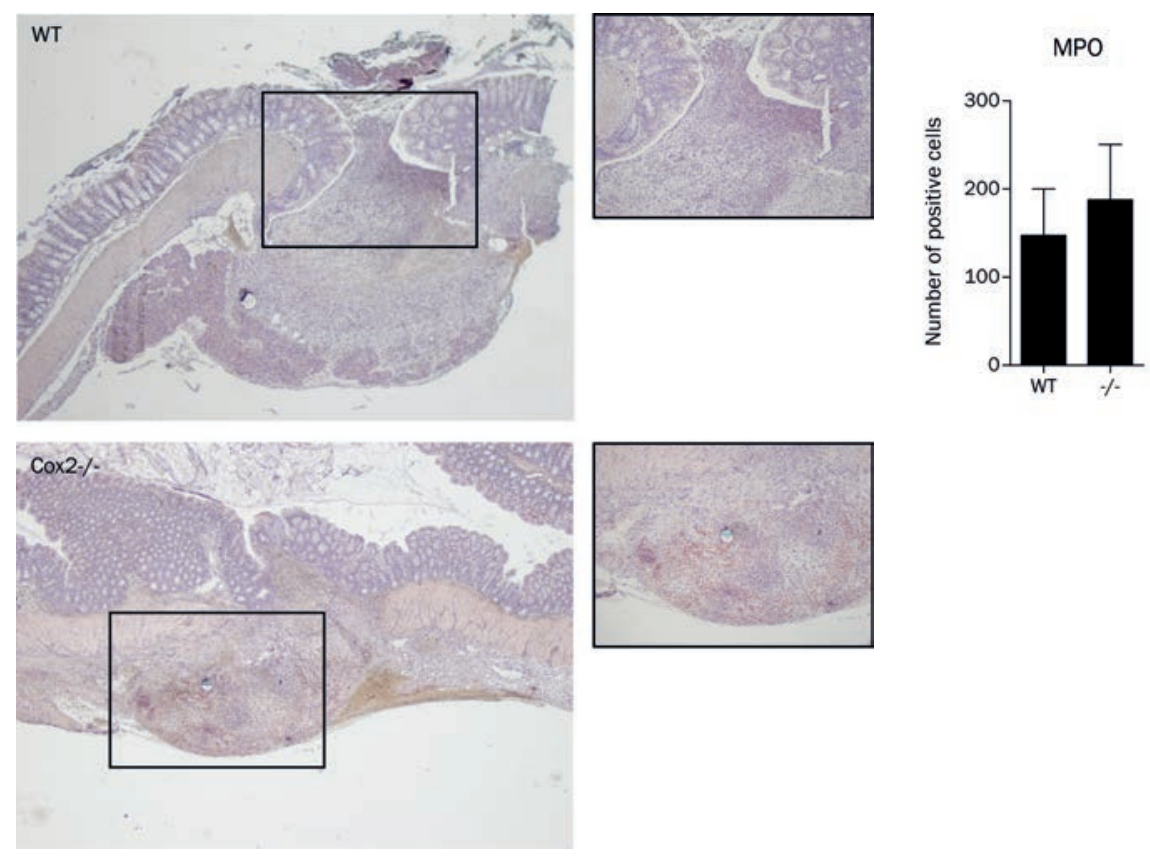

FIGURE 4. Immunohistochemical staining of MPO. A. Sections of colonic anastomotic tissue stained with MPO. Upper panel: wildtype; lower panel: COX-2-/-. Magnification 40x, inlay 100x. B. Quantification of MPO staining of wildtype and COX-2-/- mice. Data are presented as mean with SEM. We observed no differences in MPO staining between wildtype and COX-2-/- mice.

critical in the first phases of wound healing. As neutrophils are the predominant cell type in the early phase of wound healing, we assessed neutrophil influx into the colonic wound region. Interestingly, no significant differences were observed in influx of MPO-positive cells between WT and COX2\% mice (Figure 4). These data indicate that impaired inflammatory responses is not the underlying mechanism of impaired anastomotic healing in $\mathrm{COX}-2^{-1-}$ mice.

\section{Angiogenesis is impaired in anastomotic tissue of COX-2 knockout mice}

Next we hypothesized that (neo)vascularization of the wound region might be very important for adequate wound healing. COX-2 is well known to be involved in angiogenesis, and therefore we assessed whether vascularisation of the wound region was impaired in COX-2\% mice. Staining of CD31, an endothelial marker, was markedly reduced in anastomotic tissue of COX-2\% mice compared to control. Quantification of the amount of blood vessels showed that only 2 vessels $/ \mathrm{mm}^{2}$ 

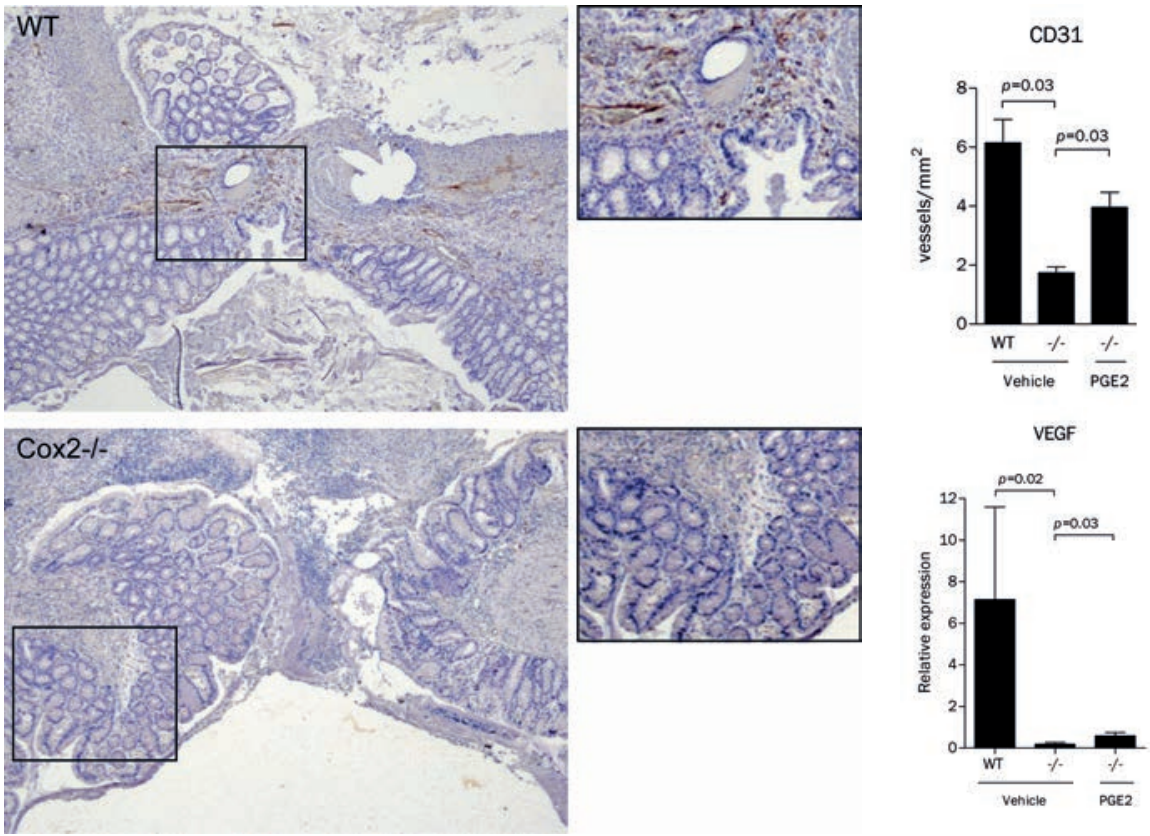

FIGURE 5. COX-2-/- mice exhibit less vascularization in colonic anastomotic tissue. A. Immunohistochemical staining of CD31 on sections of colonic anastomotic tissue. Upper panel: wildtype; lower panel: COX-2-/-. Magnification 40x, inlay 100x. B. Quantification of MPO staining of wildtype and COX-2-/- mice, and COX-2-/- mice receiving PGE2. Data are presented as mean with SEM. CD31 expression was significantly lower in COX-2-/- mice compared to wildtype mice and compared to COX-2-/- mice receiving PGE2. C. Relative expression of VEGF mRNA in colonic anastomotic tissue of wildtype and COX-2-/- mice, and COX-2-/- mice receiving PGE2. Data are presented as mean with SEM. Relative VEGF mRNA expression was significantly lower in COX-2-/- mice compared to wildtype mice and compared to COX-2-/- mice receiving PGE2.

were stained in anastomotic tissue of COX-2-1- mice compared to 6 vessels $/ \mathrm{mm}^{2}$ in wildtype mice, 5 days after surgery ( $p=0.03$, Figure $5 A-B)$. This effect could partly be reversed by administration of $\mathrm{dmPGE}_{2}$ to $\mathrm{COX}-2 \%$ mice, since significantly higher numbers of blood vessels were observed in COX-2\% mice receiving $\mathrm{dmPGE}_{2}$ compared to $\mathrm{COX}-2^{-/}$receiving vehicle (4 versus 2 vessels $/ \mathrm{mm} 2$, respectively $p=0.03$, Figure 5B). Similarly, anastomotic tissue mRNA levels of VEGF were higher in wildtype mice compared to COX-2 ${ }^{--}$mice $(p=0.02)$, and in COX-2 $\%$ mice receiving $\mathrm{dmPGE}_{2}$ compared to $\mathrm{COX}-2 \%$ mice receiving vehicle $(p=0.03)$. 


\section{Discussion}

This study shows for the first time that COX-2 plays a crucial role in gut healing after colonic surgery. Mice lacking COX-2 showed increased rates of anastomotic leakage and mortality, which could be counteracted by administering $\mathrm{PGE}_{2}$, supposedly the most important product of COX-2 in preserving gut homeostasis. Impaired angiogenesis in mice lacking COX-2 seems to be at least part of the explanation for increased anastomotic leakage rates and mortality. A correlation between use of COX-2 blocking non-steroidal anti-inflammatory drugs (NSAIDs) and impaired anastomotic healing after colorectal surgery has already been established in patients ${ }^{9,11,12}$. However, these data are of retrospective nature and a fundamental role of COX-2 in gut healing after colorectal surgery has never been established. Manieri and colleagues showed before that COX-2 is critically involved in mucosal repair after intestinal biopsies ${ }^{2}$. Furthermore, abundant evidence exists showing that COX-2 and its derived prostaglandins stimulate intestinal cancer progression through enhanced angiogenesis and proliferation and by decreasing apoptosis ${ }^{17-19}$. While these mechanisms should be counteracted to reduce cancer progression, they are indispensable in adequate wound healing. As shown by Binion et al., COX-2 and $\mathrm{PGE}_{2}$ stimulate angiogenesis through VEGF production ${ }^{8}$. Indeed, in our study mice lacking COX-2 showed significantly lower amounts of vessels in anastomotic tissue in combination with lower levels of VEGF mRNA levels, which was at least partly restored by $\mathrm{PGE}_{2}$ administration.

The current study carries several important implications. As earlier suggested by retrospective human data, the perioperative use of NSAIDs should be avoided at all times in patients undergoing colorectal surgery. This may be applicable to other conditions in which delayed gut healing is undesirable, such as chemotherapy, radiotherapy and diagnostic or therapeutic intestinal biopsies. Furthermore, patients with mutations in the COX-2 gene may be particularly vulnerable to impaired intestinal wound healing and the subsequent risk of complications after colorectal surgery or other therapeutic hits impairing gut homeostasis. Selection and personalized treatment of these patients should therefore be investigated, for example the administration of prostaglandins such as $\mathrm{PGE}_{2}$ in phases when adequate intestinal healing is crucial. Paradoxically, patients with colorectal cancer may benefit from chronic NSAID use in terms of tumor progression, while COX-2 function is highly desired in the acute phase of recovering from surgery. 
Some remarks should be made when interpreting the results of the current study. First, we only used $\mathrm{PGE}_{2}$ to counteract the effects of genetic lack of COX2. However, other prostaglandins are converted from arachidonic acid by COX-2 as well. In particular, prostacyclin $\left(\mathrm{PGI}_{2}\right)$ is expressed in colonic tissue and may therefore play a role in anastomotic healing ${ }^{2}$. This could also be explanatory for the fact that $\mathrm{PGE}_{2}$ administration did not decrease anastomotic leakage incidence in COX-2 knockout mice to wild type levels. Remarkably, all mice receiving diclofenac developed anastomotic leakage. As COX-1 is constitutively expressed in colonic tissue ${ }^{20}$, although being of less importance in acute situations such as damage and inflammation than its inducible counterpart COX-2, blockage of both isoforms may be the reason for this effect. Such high anastomotic leakage rates were not observed in previous animal studies using diclofenac 21, 22. However, a murine model has not been used before in this context. The main limitation of all animal anastomotic leakage models is that a mechanically insufficient anastomosis is constructed, which contrasts the human situation. Nonetheless, these models provide an excellent opportunity to study intestinal wound healing, providing clinical readouts such as anastomotic leakage and mortality. Finally, COX-2 and its derived prostaglandins are mainly known for their pro-inflammatory properties. Although no effect was observed on neutrophil activation in colonic tissue, inflammatory changes cannot be ruled out as part of the explanation for impaired intestinal wound healing.

In conclusion, we show that COX-2 functioning is essential for intestinal wound healing after colonic surgery, which is mediated by $\mathrm{PGE}_{2}$ production. Mice lacking COX-2 show higher rates of anastomotic leakage and mortality. In addition, angiogenesis is significantly impaired in the absence of COX-2 and PGE $\mathrm{P}_{2}$. Future research should aim at improving $C O X-2$ and $P G E_{2}$ functioning in situations when adequate intestinal wound healing is needed most, such as gastrointestinal surgery, chemotherapy and intestinal biopsies. 


\section{References}

1. Walker MR, Brown SL, Riehl TE, et al. Growth factor regulation of prostaglandinendoperoxide synthase 2 (Ptgs2) expression in colonic mesenchymal stem cells. J Biol Chem 2010;285:5026-39.

2. Manieri NA, Drylewicz MR, Miyoshi H, et al. Igf2bp1 Is Required for Full Induction of Ptgs2 mRNA in Colonic Mesenchymal Stem Cells in Mice. Gastroenterology 2012.

3. Singer, II, Kawka DW, Schloemann S, et al. Cyclooxygenase 2 is induced in colonic epithelial cells in inflammatory bowel disease. Gastroenterology 1998;115:297-306.

4. Fukata M, Chen A, Klepper A, et al. Cox-2 is regulated by Toll-like receptor-4 (TLR4) signaling: Role in proliferation and apoptosis in the intestine. Gastroenterology 2006;131:862-77.

5. Blikslager AT, Roberts MC, Rhoads JM, et al. Prostaglandins $I 2$ and E2 have a synergistic role in rescuing epithelial barrier function in porcine ileum. J Clin Invest 1997;100:1928-33.

6. Brown SL, Riehl TE, Walker MR, et al. Myd88-dependent positioning of Ptgs2expressing stromal cells maintains colonic epithelial proliferation during injury. J Clin Invest 2007;117:258-69.

7. Wang D, Dubois RN. Prostaglandins and cancer. Gut 2006;55:115-22.

8. Binion DG, Otterson MF, Rafiee P. Curcumin inhibits VEGF-mediated angiogenesis in human intestinal microvascular endothelial cells through COX-2 and MAPK inhibition. Gut 2008;57:1509-17.

9. Gorissen KJ, Benning D, Berghmans T, et al. Risk of anastomotic leakage with nonsteroidal anti-inflammatory drugs in colorectal surgery. Br J Surg 2012;99:721-7.

10. Klein M, Andersen LP, Harvald T, et al. Increased risk of anastomotic leakage with diclofenac treatment after laparoscopic colorectal surgery. Dig Surg 2009;26:27-30.

11. Klein M, Gogenur I, Rosenberg J. Postoperative use of non-steroidal anti-inflammatory drugs in patients with anastomotic leakage requiring reoperation after colorectal resection: cohort study based on prospective data. BMJ 2012;345:e6166.

12. Holte $\mathrm{K}$, Andersen J, Jakobsen $\mathrm{DH}$, et al. Cyclo-oxygenase 2 inhibitors and the risk of anastomotic leakage after fast-track colonic surgery. Br J Surg 2009;96:650-4.

13. Burton TP, Mittal A, Soop M. Nonsteroidal anti-inflammatory drugs and anastomotic dehiscence in bowel surgery: systematic review and meta-analysis of randomized, controlled trials. Dis Colon Rectum 2013;56:126-34.

14. Gustafsson UO, Scott MJ, Schwenk W, et al. Guidelines for perioperative care in elective colonic surgery: Enhanced Recovery After Surgery (ERAS(R)) Society recommendations. Clin Nutr 2012;31:783-800.

15. Nygren J, Thacker J, Carli F, et al. Guidelines for perioperative care in elective rectal/ pelvic surgery: Enhanced Recovery After Surgery (ERAS(R)) Society recommendations. Clin Nutr 2012;31:801-16.

16. Komen N, van der Wal HC, Ditzel M, et al. Colorectal anastomotic leakage: a new experimental model. J Surg Res 2009;155:7-12. 
17. Liao X, Lochhead P, Nishihara R, et al. Aspirin use, tumor PIK3CA mutation, and colorectal-cancer survival. N Engl J Med 2012;367:1596-606.

18. Rothwell PM, Fowkes FG, Belch JF, et al. Effect of daily aspirin on long-term risk of death due to cancer: analysis of individual patient data from randomised trials. Lancet 2011;377:31-41.

19. Wang D, Dubois RN. Eicosanoids and cancer. Nat Rev Cancer 2010;10:181-93.

20. Tessner TG, Cohn SM, Schloemann S, et al. Prostaglandins prevent decreased epithelial cell proliferation associated with dextran sodium sulfate injury in mice. Gastroenterology 1998;115:874-82.

21. Klein M, Krarup PM, Burcharth J, et al. Effect of diclofenac on cyclooxygenase-2 levels and early breaking strength of experimental colonic anastomoses and skin incisions. Eur Surg Res 2011;46:26-31.

22. Inan A, Koca C, Sen M. Effects of diclofenac sodium on bursting pressures of anastomoses and hydroxyproline contents of perianastomotic tissues in a laboratory study. Int J Surg 2006;4:222-7. 


Chapter 4. Functional compromise reflected by sarcopenia, frailty and nutritional depletion predicts adverse postoperative outcome after colorectal cancer surgery

Kostan W. Reisinger, Jeroen L.A. van Vugt, Juul J.W. Tegels, Claire Snijders, Karel W.E. Hulsewé, Anton G.M. Hoofwijk, Jan H.M.B. Stoot, Maarten F. Von Meyenfeldt, Geerard L. Beets, Joep P.M. Derikx, Martijn Poeze. Annals of Surgery 2015. 261(2):345-52 


\section{Abstract \\ Objective}

To determine the association of sarcopenia with postoperative morbidity and mortality after colorectal surgery.

\section{Summary background data}

Functional compromise in elderly colorectal surgical patients is considered as a significant factor of impaired postoperative recovery. Therefore, the predictive value of pre-operative functional compromise assessment was investigated. Sarcopenia is a hallmark of functional compromise.

\section{Methods}

310 consecutive patients who underwent oncologic colorectal surgery were enrolled in a prospective digital database. Sarcopenia was assessed using the L3 muscle index using Osirix ${ }^{\circledR}$ on pre-operative computed tomography (CT). Groningen Frailty Indicator (GFI) and Short Nutritional Assessment Questionnaire (SNAQ) scores were used to assess frailty and nutritional compromise. Predictors for anastomotic leakage, sepsis and mortality were analyzed by logistic regression analysis.

\section{Results}

Age was an independent predictor of mortality ( $p=0.04$; odds ratio (OR), 1.17; 95\% confidence interval (Cl), 1.01 - 1.37). 30-day/in-hospital mortality rate in sarcopenic patients was $8.8 \%$ versus $0.7 \%$ in non-sarcopenic patients $(p=0.001$; OR 15.5; 95\% Cl, 2.00 - 120). Sarcopenia was not predictive for anastomotic leakage or sepsis. Combination of high SNAQ score, high GFI score and sarcopenia strongly predicted sepsis ( $p=0.001$; OR, 25.1; 95\% CI, 5.11 - 123), sensitivity, 46\%; specificity, 97\%; positive likelihood ratio, 13 (95\% Cl, 4.4 - 38); negative likelihood ratio, 0.57 (95\% $\mathrm{Cl}, 0.33-0.97)$.

\section{Conclusion}

Functional compromise in colorectal cancer surgery is associated with adverse postoperative outcome. Assessment of functional compromise by means of a nutritional questionnaire (SNAQ), a frailty questionnaire (GFI), and sarcopenia measurement (L3 muscle index) can accurately predict postoperative sepsis. 


\section{Introduction}

With an increasingly ageing population, the number of older cancer patients is concomitantly rising. Currently 50 percent of patients with colorectal cancer is aged 70 years or older ${ }^{1}$. Older patients are at increased risk for developing perioperative complications and suffer from higher mortality rates ${ }^{2}$. Postoperative recovery plays a crucial role in cancer treatment with respect to survival, morbidity, and quality of life ${ }^{3-5}$. Hence, pre-operative assessment is important to identify patients at risk for developing postoperative complications. Widely accepted risk assessments are however considered to be subjective and imprecise and not focused on patients with cancer ${ }^{6}$.

It is hypothesized that functional compromise in the older surgical patient is a significant predictor of postoperative complications ${ }^{7,8}$. A widely investigated aspect of functional compromise is frailty. Frailty is a state of increased vulnerability towards stressors in older individuals, leading to an increased risk of developing adverse health outcomes ${ }^{9}$. However, the definition of frailty remains controversial and there is no consensus about the clinical use of frailty as a preoperative predictor for postoperative outcome in elderly patients ${ }^{7}$. Weight loss, low muscle strength, reduced physical activity, exhaustion, and decreased walking speed are elements of a physical definition of frailty 6,8 , whereas comorbidity, polypharmacy, physical functioning, nutritional and cognitive status, depression and social support are a more multidimensional tool to assess frailty ${ }^{10,11}$. In addition, sarcopenia is an important factor in functional compromise and depletion of skeletal muscle can occur in normal-weight, overweight and obese patients and does therefore not equal ordinary weight loss or cachexia ${ }^{12,13}$. Malnutrition is another key element of functional compromise that is associated with poor clinical outcome ${ }^{14}$. In elderly patients, preoperative malnutrition is common, and worsens the condition of this already vulnerable population ${ }^{15}$.

In this study we investigated whether functional compromise is associated with postoperative morbidity and mortality after colorectal surgery. The following parameters of functional compromise were evaluated: CT-based measurement of muscle mass, Groningen Frailty Index (GFI) ${ }^{16}$ and Short Nutritional Assessment Questionnaire (SNAQ) ${ }^{17}$. 


\section{Materials and methods}

\section{Patients}

All consecutive patients who underwent oncologic colorectal surgery in a single non-academic center with a dedicated team of colorectal surgeons from January 2010 until May 2012 were enrolled in a prospective digital cohort and analyzed retrospectively. The only exclusion criterion was unavailability of a preoperative CT scan. Data in the database included characteristics of the primary tumor and oncologic staging, specifications of surgical treatment, chemotherapy, radiotherapy, and postoperative complications and included a preoperative CTscan of the abdomen which was performed routinely for tumor staging.

\section{Complications}

The following complications were analyzed prospectively in detail: anastomotic leakage (surgically and/or radiologically) and/or intra-abdominal abscess diagnosed by CT examination or treated by percutaneous drainage or surgery, sepsis and septic shock (defined as two or more of the following criteria positive: (1) temperature $>38^{\circ} \mathrm{C}$ or $<36^{\circ} \mathrm{C}$; (2) heart rate $>90 \mathrm{bpm}$; (3) respiratory rate $>20$ breaths/min or $\mathrm{PaCO}_{2}<32 \mathrm{mmHg}$; (4) white blood cell count $>12 \times 10^{9} / \mathrm{L}$, $<4 \times 10^{9} / \mathrm{L}$, or $>10 \%$ immature (band) forms plus documented infection and hypotension despite adequate fluid resuscitation (in case of septic shock) ${ }^{18}$ ), and postoperative mortality (within 30 days postoperatively or within same period of hospital admission). The 30-day mortality for patients discharged within this period was verified by checking the municipal personal records database.

\section{Pre-operative CT-based muscle measurements}

All patients underwent a CT-scan of the abdomen as part of routine pre-operative assessment, which was delivered on CD-ROM or DVD. Measurements were performed using Osirix ${ }^{\circledR}$ Version 3.3 (32-bit; http://www.osirix-viewer.com). The cross-sectional skeletal muscle surface $\left(\mathrm{cm}^{2}\right)$ assessment of sarcopenia was performed at the level of the third lumbar vertebra (L3) on two consecutive transversal coupes on which both vertebral spines were visible ${ }^{19}$. The 'Grow Region (2D/3D Segmentation)' tool in the menu of the program facilitated to automatically select all skeletal muscle mass in one coupe. The distinction between different tissues is based on Hounsfield Units (HU). A threshold range 
of $-30 \mathrm{HU}$ to $+110 \mathrm{HU}$ was used for skeletal muscle. Muscles measured were: psoas, paraspinal, transverse abdominal, external oblique, internal oblique and rectus abdominis muscles (Figure 1). Hand-adjustment of the selected areas was performed if necessary and the muscle area was calculated automatically ${ }^{19}$. The averages of the two measurements were used for calculations. Two investigators independently measured all L3-muscle area surface parameters (J.v.V. and J.T.). A third investigator (K.R.) performed a random control measurement on $10 \%$ of the CT-scans. All investigators did not have specific skills in radiology.

\section{Sarcopenia}

The L3-muscle area surfaces were normalized for patient height to calculate the L3-muscle index and expressed in $\mathrm{cm}^{2} / \mathrm{m}^{2}$. The cut-off values used for sarcopenia were $52.4 \mathrm{~cm}^{2} / \mathrm{m}^{2}$ for men and $38.5 \mathrm{~cm}^{2} / \mathrm{m}^{2}$ for women, based on the method of Prado et al. ${ }^{13}$.

\section{Groningen Frailty Indicator (GFI)}

The GFI has been developed as a simple screening instrument for frailty ${ }^{16}$. The GFI screens on physical, cognitive, social and emotional items (Appendix 1). The maximum score is 15 points. Patients scoring 5 or more points were considered frail ${ }^{16}$. A trained nurse routinely performed GFI-scores at pre-operative consultation or hospital admission in patients aged 70 or older.

\section{Short Nutritional Assessment Questionnaire (SNAQ)}

The SNAQ is a valid and reproducible tool to detect malnourished hospitalized patients without the need to calculate percentage weight loss or BMI ${ }^{17}$. The maximum score is 5 points (Appendix 2). Patients with a score of 3 points or more on the SNAQ were classified as malnourished (requiring nutritional support and supervision by a dietician) ${ }^{17}$. A trained nurse routinely performed SNAQ-scores at pre-operative consultation or hospital admission.

\section{Statistical analysis}

Frequencies are presented as absolute numbers and percentages. Continuous data are presented as mean (standard error of the mean [SEM]). Normality was tested using Kolmogorov-Smirnov. Differences between groups were analyzed 
with the Pearson chi-square test for dichotomous parameters. Odds ratios and 95\% confidence intervals were calculated by logistic regression analysis. For the calculation of significant predictors of mortality and complications, univariate analyses with clinically relevant parameters were performed. Significant predictors $(p<0.05)$ or predictors showing a trend towards significance $(0.05 \leq p<0.20)$ based on univariate analysis were entered into multivariate logistic regression analysis. Interactions between sarcopenia, GFI scores and SNAQ scores were tested. Functional compromise was defined as sarcopenia, high GFI score and high SNAQ score. Diagnostic accuracy of functional compromise assessment to predict postoperative complications was evaluated by calculating sensitivity, specificity, positive likelihood ratio (LR+), and negative likelihood ratio (LR-) using crosstabs. The interobserver agreement (J.v.V., J.T., K.R.) of the L3 muscle index assessment of sarcopenia was analyzed by Pearson's correlation and Cohen's kappa coefficient. Two-tailed p-values $<0.05$ were considered significant. All statistical analyses were performed using SPSS® 20.0 (SPSS Inc, Chicago, IL, USA). 


\section{Results}

\section{Patients}

340 patients were enrolled in the prospective database. In 30 patients, no staging CT scan was available, for which the main reason was emergency surgery. Therefore 310 patients undergoing both elective and acute colorectal resection were included in the analyses of the current study. The patient characteristics are listed in Table 1. Of these 310 patients, 148 (47.7\%) were identified as sarcopenic as defined by muscle index at L3 level; 90/155 (58.1\%) males and 58/155 (37.4\%) females. Mean L3 muscle indexes for males and females were $51.5(0.65) \mathrm{cm}^{2} /$ $\mathrm{m}^{2}$ and $40.7(0.53) \mathrm{cm}^{2} / \mathrm{m}^{2}$, respectively. Of patients aged 70 or older, $41(24.6 \%)$ were frail as defined by a GFI score of 5 or more. SNAQ scores were 3 points or higher in 36 (11\%) of all patients.

\section{Interobserver agreement of CT-based muscle measurement by Osirix ${ }^{\circledR}$}

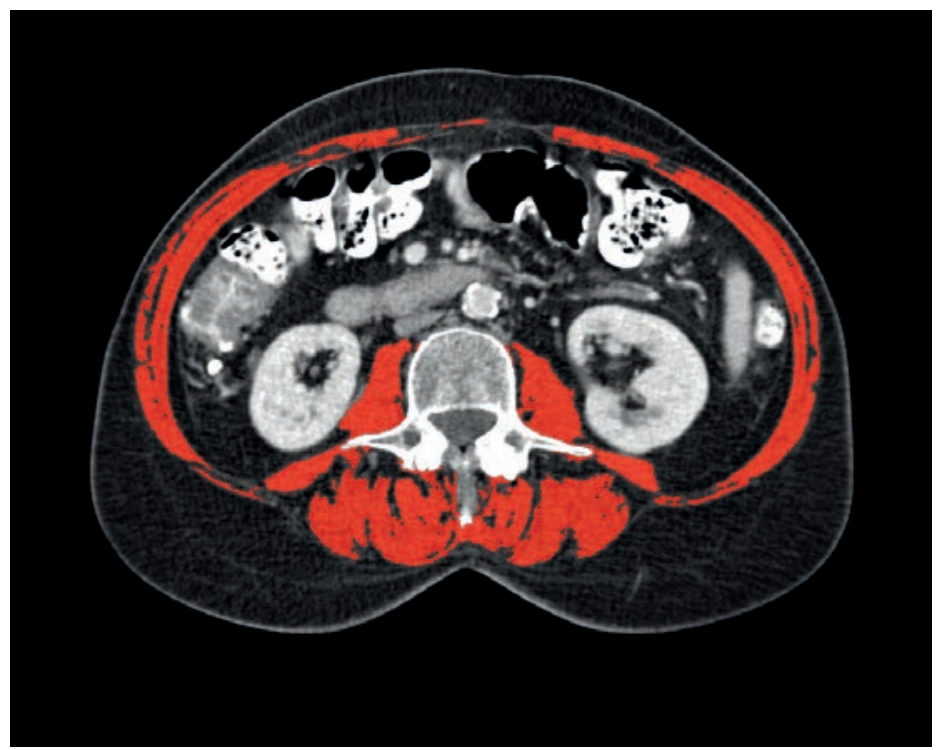

FIGURE 1. Computed tomography image at the third lumbar vertebral level. The following skeletal muscles are outlined in red: psoas, paraspinal, transverse abdominal, external oblique, internal oblique and rectus abdominis muscles. This female sarcopenic patient had an L3 muscle index of $34.3 \mathrm{~cm}^{2} / \mathrm{m}^{2}$. 
Two independent investigators measured the L3 muscle areas of all patients, showing a strong and significant correlation $\left(R^{2}=0.98 ; p<0.0001\right)$. BlandAltman analysis produced 95\% limits of agreement, $-5.9 \%-8.7 \%$. The kappa (K) of sarcopenia assessment by CT image analysis using Osirix ${ }^{\circledR}$ was 0.87 (95\% confidence interval $(\mathrm{Cl}), 0.82$ - 0.93). The random control measurement on $10 \%$ of the CT-scans by a third investigator yielded excellent correlation $\left(R^{2}=0.98\right.$; $p<0.0001)$ and a kappa of 0.74 (95\% Cl, $0.46-1.00)$.

\section{Mortality}

Fourteen patients died within 30 days or during hospital admission (4.5\%). Causes of mortality are listed in Table 2. In univariate analyses sarcopenia $(p=0.009)$, male gender $(p=0.02)$, age $(p=0.002)$, epidural analgesia $(p=0.14)$, medical history of abdominal surgery $(p=0.02)$, ASA classification $(p=0.02)$, and stage 3-4

TABLE 1. Patient characteristics

\begin{tabular}{|c|c|c|c|}
\hline & & $\begin{array}{l}\text { Number of patients } \\
(\%)\end{array}$ & Mean (SEM) \\
\hline \multirow[t]{2}{*}{ Sex } & Male & $155(50.0)$ & \\
\hline & Female & $155(50.0)$ & \\
\hline \multirow[t]{2}{*}{ Age (years) } & & & $69(0.6)^{b}$ \\
\hline & $>70$ & 159 (51.3) & \\
\hline \multirow[t]{2}{*}{$\mathrm{BMI}\left(\mathrm{kg} / \mathrm{m}^{2}\right)$} & & & $26.3(0.26)^{b}$ \\
\hline & $>25$ & $182(58.7)$ & \\
\hline \multicolumn{2}{|l|}{$\begin{array}{l}\text { Length of hospital stay } \\
\text { (days) }\end{array}$} & & $11(0.6)^{b}$ \\
\hline \multirow[t]{4}{*}{ ASA } & I & $17(5.5)$ & \\
\hline & II & 219 (70.6) & \\
\hline & III & $62(20.0)$ & \\
\hline & IV & $12(3.9)$ & \\
\hline \multicolumn{2}{|l|}{ Laparoscopy } & 33 (10.6) & \\
\hline \multicolumn{2}{|l|}{ Primary anastomosis } & 249 (80.3) & \\
\hline \multirow{2}{*}{ Tumor location } & Colon & 205 (66.1) & \\
\hline & Rectum & 105 (33.9) & \\
\hline \multirow[t]{4}{*}{ Complications } & $\begin{array}{l}\text { Anastomotic leakage } \\
\text { and /or abscess }\end{array}$ & $37(14.9)$ & \\
\hline & Sepsis & $14(4.5)$ & \\
\hline & Death & $14(4.5)$ & \\
\hline & Cause-related death & $8(2.6)$ & \\
\hline
\end{tabular}


disease $(p=0.14)$ were significant predictors of postoperative mortality or showed a trend towards significance. Multivariate logistic regression revealed male gender (OR, 49.29 (95\% Cl, 2.48 - 978.4); $p=0.01)$, sarcopenia (OR, 43.30 (95\% $\mathrm{Cl}, 2.74$ - 685.2); $p=0.007)$, age (OR, 1.17; 95\% Cl, 1.01 - 1.37; $p=0.04)$, and medical history of abdominal surgery (OR, 31.16 (3.09 - 313.9); $p=0.004$ ) being independent predictors of mortality. Logistic regression results are summarized in Table 3A.

To evaluate the effect of functional compromise on mortality in more detail, interactions between $S N A Q \geq 3, G F I \geq 5$, and sarcopenia were studied. None of the interactions increased the predictive value of sarcopenia.

\section{Anastomotic leakage}

The incidence of anastomotic leakage and/or intra-abdominal abscess was 37 (14.9\%) in patients with a primary anastomosis. Mortality within this group was 9.3\%. Significant predictors or predictors showing a trend for anastomotic leakage and/or intra-abdominal abscess were sarcopenia ( $p=0.13)$, SNAQ $\geq 3(p=0.08)$, medical history of abdominal surgery $(p=0.06)$, surgery of the rectum $(p=0.02)$, open surgery $(p=0.10)$, stapled anastomosis $(p=0.06)$, stage $3-4$ disease $(p=0.04)$, and need for blood transfusion $(p<0.001)$. In multivariate analysis, stage 3-4 disease (OR, 3.68; 95\% Cl, 1.18 - 11.4; $p=0.02$ ) and need for blood TABLE 2. Causes of mortality

\begin{tabular}{lll}
\hline \multicolumn{2}{c}{ Cause } & Postoperative day of mortality \\
\hline Sarcopenic patients $(\mathrm{n}=13)$ & \\
1 & Pneumonia, sepsis, cardiorespiratory failure & 47 \\
2 & Sepsis of surgical site origin & 5 \\
3 & Sepsis of surgical site origin & 2 \\
4 & Sepsis of surgical site origin & 2 \\
5 & Cardiorespiratory failure after anastomotic & 25 \\
& leakage & \\
6 & Pneumonia & 30 \\
7 & Sepsis of surgical site origin & 7 \\
8 & Pneumonia & 3 \\
9 & Cardiorespiratory failure & 8 \\
10 & Pneumonia & 7 \\
11 & Unknown cause, probably pneumonia & 35 \\
12 & Intraoperative presacral bleeding & 0 \\
13 & Gastric hemorrhage & 10 \\
Non-sarcopenic patients ( $=1$ ) & \\
14 & Anastomotic leakage & 25 \\
\hline
\end{tabular}


transfusion (OR, 7.81; 95\% Cl, 2.81 - 21.8; $p=0001$ ) were independent predictors of anastomotic leakage and/or intra-abdominal abscess. Logistic regression results are summarized in Table 3B.

To evaluate the effect of functional compromise on anastomotic leakage in more detail, interactions between $\mathrm{SNAQ} \geq 3, \mathrm{GFI} \geq 5$, and sarcopenia were studied. None of the interactions were significantly predictive of anastomotic leakage.

\section{Sepsis}

Fourteen patients (4.5\%) developed sepsis. Mortality within this group was $36 \%$. Univariate analysis demonstrated that GFI $\geq 5 \quad(p=0.03)$, SNAQ $\geq 3 \quad(p=0.003)$, age $(p=0.03)$, epidural analgesia $(p=0.14)$, and medical history of abdominal surgery $(p=0.17)$ were significant predictors of sepsis or showed a trend towards significance. In multivariate logistic regression analysis, SNAQ $\geq 3$ was independently predictive of postoperative sepsis (OR, 4.37; 95\% Cl, 1.07 - 17.9; $p=0.04)$. Logistic regression results are summarized in Table $3 \mathrm{C}$.

To evaluate the effect of functional compromise on sepsis in more detail, interactions between SNAQ $\geq 3, \mathrm{GFI} \geq 5$, and sarcopenia were studied. The interaction between SNAQ $\geq 3$ and GFI $\geq 5$ compared to SNAQ $\geq 3$ alone increased the capability of predicting postoperative sepsis (OR, 13.1; 95\% Cl, $3.02-57.2$; $p=0.001$ ). The interaction between SNAQ $\geq 3, \mathrm{GFI} \geq 5$, and sarcopenia further increased the risk of sepsis (OR, 25.1; 95\% Cl, 5.11 - 123; $p=0.001)$.

\section{Diagnostic accuracy of SNAQ/GFI/sarcopenia score to predict sepsis}

As a strong association of functional compromise (the combination of high SNAQ, high GFI, and sarcopenia) with sepsis was shown by the previous data, the clinical applicability of this functional compromise assessment needed further exploration. Therefore, the diagnostic accuracy of (pre-operative) functional compromise assessment to predict postoperative sepsis was investigated. A positive functional compromise test was defined as $\mathrm{SNAQ} \geq 3, \mathrm{GFI} \geq 5$, and sarcopenia (L3 muscle index $<52.4 \mathrm{~cm}^{2} / \mathrm{m}^{2}$ for men, and $<38.5 \mathrm{~cm}^{2} / \mathrm{m}^{2}$ for women). This resulted in sensitivity, 46\%; specificity, 97\%; LR+, 13 (95\% Cl, 4.4 - 38); LR-, 0.57 (95\% Cl, 0.33 - 0.97). Subgroup analysis of patients undergoing rectal surgery revealed a higher diagnostic accuracy; sensitivity, 67\%; specificity, 98\%; LR+, 29 (95\% Cl, 3.61 - 239); LR-, 0.34 (95\% Cl, 0.07 - 1.69). 
TABLE 3A. Logistic regression analysis of mortality

\begin{tabular}{|c|c|c|c|c|c|}
\hline & \multirow[b]{2}{*}{ Mortality } & \multicolumn{2}{|l|}{ Univariate analysis } & \multicolumn{2}{|l|}{ Multivariate analysis } \\
\hline & & Odds ratio & $\mathbf{P}$ & Odds ratio & $\mathbf{P}$ \\
\hline \multicolumn{6}{|c|}{ Sarcopenia } \\
\hline No & $1 / 162$ & 1 & & 1 & \\
\hline Yes & $13 / 148$ & $15.50(2.00-120.0)$ & 0.009 & $43.30(2.74-685.2)$ & 0.007 \\
\hline \multicolumn{6}{|l|}{$\mathrm{GFI} \geq 5$} \\
\hline No & $8 / 114$ & 1 & & & \\
\hline Yes & $3 / 39$ & $0.68(0.09-5.45)$ & 0.72 & & \\
\hline \multicolumn{6}{|l|}{$S N A Q \geq 3$} \\
\hline No & $8 / 185$ & 1 & & & \\
\hline Yes & $4 / 112$ & $0.82(0.24-2.79)$ & 0.75 & & \\
\hline \multicolumn{6}{|l|}{ Gender } \\
\hline Female & $2 / 155$ & 1 & & 1 & \\
\hline Male & $12 / 155$ & 6.42 (1.41-29.18) & 0.02 & $49.29(2.48-978.4)$ & 0.01 \\
\hline Age & & $1.12(1.04-1.20)$ & 0.002 & $1.17(1.01-1.37)$ & 0.04 \\
\hline \multicolumn{6}{|l|}{ Epidural } \\
\hline No & $8 / 117$ & 1 & & & \\
\hline Yes & $6 / 193$ & $0.44(0.15-1.29)$ & 0.14 & & \\
\hline \multicolumn{6}{|c|}{ Previous abdominal surgery } \\
\hline No & $5 / 208$ & 1 & & 1 & \\
\hline Yes & 9/102 & $3.93(1.28-12.05)$ & 0.02 & $31.16(3.09-313.9)$ & 0.004 \\
\hline \multicolumn{6}{|l|}{ ASA } \\
\hline I & $0 / 17$ & 1 & 0.02 & & \\
\hline ॥ & $5 / 219$ & $\infty(0.00-\infty)$ & 1.00 & & \\
\hline III & $8 / 62$ & $\infty(0.00-\infty)$ & 1.00 & & \\
\hline IV & $1 / 12$ & $\infty(0.00-\infty)$ & 1.00 & & \\
\hline \multicolumn{6}{|c|}{ Disease stage } \\
\hline 1-2 & $3 / 107$ & 1 & & & \\
\hline $3-4$ & $9 / 122$ & $2.76(0.73-10.48)$ & 0.14 & & \\
\hline \multicolumn{6}{|c|}{$\mathrm{BMI}>25 \mathrm{~kg} / \mathrm{m}^{2}$} \\
\hline No & $8 / 128$ & 1 & & & \\
\hline Yes & $6 / 182$ & $0.51(0.17-1.51)$ & 0.23 & & \\
\hline \multicolumn{6}{|l|}{ Smoking } \\
\hline No & $4 / 170$ & 1 & & & \\
\hline Yes & $8 / 140$ & $2.12(0.62-7.21)$ & 0.23 & & \\
\hline \multicolumn{6}{|l|}{ Diabetes } \\
\hline No & $11 / 252$ & 1 & & & \\
\hline Yes & $3 / 58$ & $1.20(0.32-4.43)$ & 0.79 & & \\
\hline
\end{tabular}

Definition of mortality: 30-day mortality and/or in-hospital mortality Values in parentheses are 95\% confidence intervals 
TABLE 3B. Logistic regression analysis of anastomotic leakage

\begin{tabular}{|c|c|c|c|c|c|}
\hline & \multirow[b]{2}{*}{ Leakage } & \multicolumn{2}{|l|}{ Univariate analysis } & \multicolumn{2}{|c|}{ Multivariate analysis } \\
\hline & & Odds ratio & $\mathbf{P}$ & Odds ratio & $\mathbf{P}$ \\
\hline \multicolumn{6}{|l|}{ Sarcopenia } \\
\hline No & $24 / 133$ & 1 & & & \\
\hline Yes & $13 / 116$ & $0.57(0.28-1.19)$ & 0.13 & & \\
\hline \multicolumn{6}{|l|}{$\mathrm{GFI} \geq 5$} \\
\hline No & $12 / 92$ & 1 & & & \\
\hline Yes & $5 / 30$ & $1.33(0.42-4.15)$ & 0.62 & & \\
\hline \multicolumn{6}{|l|}{$S N A Q \geq 3$} \\
\hline No & $21 / 147$ & 1 & & & \\
\hline Yes & $14 / 91$ & $2.33(0.90-6.00)$ & 0.08 & & \\
\hline \multicolumn{6}{|l|}{ Gender } \\
\hline Female & $16 / 126$ & 1 & & & \\
\hline Male & $21 / 123$ & $1.42(0.70-2.86)$ & 0.33 & & \\
\hline Age & & $0.99(0.96-1.02)$ & 0.57 & & \\
\hline \multicolumn{6}{|c|}{ Previous abdominal surgery } \\
\hline No & $20 / 168$ & 1 & & & \\
\hline Yes & $17 / 81$ & $1.97(0.97-4.00)$ & 0.06 & & \\
\hline \multicolumn{6}{|l|}{ Disease stage } \\
\hline $1-2$ & $6 / 97$ & 1 & & 1 & \\
\hline $3-4$ & $17 / 109$ & 2.80 (1.06-7.43) & 0.04 & $3.68(1.18-11.4)$ & 0.02 \\
\hline \multicolumn{6}{|l|}{ Tumor location } \\
\hline Colon & $22 / 188$ & 1 & & & \\
\hline Rectum & $15 / 61$ & $2.46(1.18-5.12)$ & 0.02 & & \\
\hline \multicolumn{6}{|l|}{ Type of surgery } \\
\hline Laparoscopy & $1 / 29$ & 1 & & & \\
\hline Laparotomy & $36 / 220$ & $5.48(0.72-41.56)$ & 0.10 & & \\
\hline \multicolumn{6}{|l|}{ Anastomosis } \\
\hline Manual & $15 / 137$ & 1 & & & \\
\hline Stapled & $21 / 106$ & $2.01(0.98-4.12)$ & 0.06 & & \\
\hline \multicolumn{6}{|c|}{ Blood transfusion } \\
\hline No & $19 / 207$ & 1 & & 1 & \\
\hline Yes & $18 / 42$ & 7.42 (3.43-16.06) & $<0.001$ & $7.81(2.81-21.8)$ & $<0.001$ \\
\hline \multicolumn{6}{|l|}{ Smoking } \\
\hline No & $17 / 133$ & 1 & & & \\
\hline Yes & $20 / 116$ & $1.47(0.70-3.08)$ & 0.30 & & \\
\hline \multicolumn{6}{|l|}{ Diabetes } \\
\hline No & $31 / 203$ & 1 & & & \\
\hline Yes & $6 / 46$ & $0.83(0.33-2.13)$ & 0.70 & & \\
\hline
\end{tabular}

Anastomotic leakage and/or intra-abdominal abscess in 249 patients with primary anastomosis. Values in parentheses are 95\% confidence intervals 
TABLE 3C. Logistic regression analysis of sepsis

\begin{tabular}{|c|c|c|c|c|c|}
\hline & \multirow[b]{2}{*}{ Sepsis rate } & \multicolumn{2}{|l|}{ Univariate analysis } & \multicolumn{2}{|c|}{ Multivariate analysis } \\
\hline & & Odds ratio & $\mathbf{P}$ & Odds ratio & $\mathbf{P}$ \\
\hline \multicolumn{6}{|c|}{ Sarcopenia } \\
\hline No & $6 / 162$ & 1 & & & \\
\hline Yes & $8 / 148$ & 1.49 (0.50-4.39) & 0.47 & & \\
\hline \multicolumn{6}{|l|}{$\mathrm{GFI} \geq 5$} \\
\hline No & $5 / 114$ & 1 & & & \\
\hline Yes & $6 / 39$ & 3.96 (1.14-13.83) & 0.03 & & \\
\hline \multicolumn{6}{|l|}{$S N A Q \geq 3$} \\
\hline No & $6 / 185$ & 1 & & 1 & \\
\hline Yes & $6 / 112$ & $6.17(1.84-20.64)$ & 0.003 & $4.37(1.07-17.9)$ & 0.04 \\
\hline \multicolumn{6}{|l|}{ Gender } \\
\hline Female & $8 / 155$ & 1 & & & \\
\hline Male & $6 / 155$ & $0.74(0.25-2.19)$ & 0.59 & & \\
\hline Age & & 1.07 (1.01-1.14) & 0.03 & & \\
\hline \multicolumn{6}{|l|}{ Epidural } \\
\hline No & $8 / 117$ & 1 & & & \\
\hline Yes & $6 / 193$ & $0.44(0.15-1.29)$ & 0.14 & & \\
\hline \multicolumn{6}{|c|}{ Previous abdominal surgery } \\
\hline No & $7 / 208$ & 1 & & & \\
\hline Yes & $7 / 102$ & $2.12(0.72-6.20)$ & 0.17 & & \\
\hline \multicolumn{6}{|c|}{ Disease stage } \\
\hline $1-2$ & $4 / 107$ & 1 & & & \\
\hline $3-4$ & $4 / 122$ & $0.87(0.21-3.58)$ & 0.85 & & \\
\hline \multicolumn{6}{|l|}{ ASA } \\
\hline I & $0 / 17$ & 1 & 0.95 & & \\
\hline ॥ & $10 / 219$ & $\infty(0.00-\infty)$ & 1.00 & & \\
\hline III & $4 / 62$ & $\infty(0.00-\infty)$ & 1.00 & & \\
\hline IV & $0 / 12$ & $1.00(0.00-\infty)$ & 1.00 & & \\
\hline \multicolumn{6}{|l|}{ Smoking } \\
\hline No & $7 / 170$ & 1 & & & \\
\hline Yes & $7 / 140$ & $1.21(0.40-3.70)$ & 0.74 & & \\
\hline \multicolumn{6}{|l|}{ Diabetes } \\
\hline No & $11 / 252$ & 1 & & & \\
\hline Yes & $3 / 58$ & $1.62(0.50-5.29)$ & 0.42 & & \\
\hline
\end{tabular}

Values in parentheses are 95\% confidence intervals 


\section{Discussion}

The association of functional compromise and unfavorable postoperative outcome in colorectal surgery has been described before ${ }^{8,10}$. Robinson and coworkers investigated frailty, an important aspect of functional compromise, using a scoring system based on 7 items of different domains and showed that frailty increased healthcare costs and 30-day readmission rates. However, postoperative complications were not specified ${ }^{10}$. Another study involving elderly colorectal surgery patients demonstrated a 4-fold increased risk for major postoperative complications when frailty was present ${ }^{8}$. The frailty assessment used was based on the criteria of Fried et al. ${ }^{20}$, i.e. 3 or more items of the following: unintentional weight loss (10 lbs in past year), self-reported exhaustion, weakness (grip strength), slow walking speed, and low physical activity. The current study underlines and extends the abovementioned findings for several reasons. First, this is the largest cohort thus far addressing the influence of functional compromise including frailty on postoperative outcome in colorectal surgery. Second, the proposed functional compromise assessment is easy to perform as staging CT scans are routinely performed and SNAQ and GFI questionnaires can be completed in only few minutes time. In most Dutch hospitals, nutritional assessment and GFI assessments are part of standard care at hospital admission. Third, this study highlights metabolic and functional compromise to be associated with postoperative mortality in colorectal surgery.

As in the present study, elderly patients showed higher rates of short-term postoperative mortality and sepsis, pointing at a the role of sepsis causing postoperative death in these patients. Moreover, 9 out of 13 mortality cases in sarcopenic patients were caused by sepsis or pneumonia. In the context of major abdominal surgery, it could be assumed that sepsis may be the consequence of inadequate gut barrier function, especially in the cancer bearing host. Loss of intestinal barrier function is indeed correlated with sepsis ${ }^{21-24}$. In addition, it has been demonstrated that gut barrier dysfunction and endotoxemia develop concurrently with cachexia in a mouse model of colorectal neoplasia ${ }^{25}$. In the current study, low muscle mass strongly correlated with mortality, however the link with sepsis could not be made statistically. It could be hypothesized that sarcopenia reflects a state of prolonged catabolism impairing host immune function and leading to an inadequate response to inflammatory stimuli. A strong association with sepsis was found when muscle mass assessment was combined with SNAQ and GFI scores, indicating that muscle wasting as a reflection of the 
more comprehensive syndrome of functional compromise predicts postoperative morbidity. Although an elevated inflammatory response has been observed in sarcopenic colorectal cancer patients ${ }^{26}$, future studies should address the unraveled link between frailty, gut barrier function and development of sepsis.

A correlation between elevated serum markers of preoperative systemic inflammatory response and postoperative infectious complications and mortality has been established extensively 27, 28. Specifically, the Glasgow Prognostic Score (GPS) comprising C-reactive protein (CRP) and albumin levels is an easily obtainable and accurate scoring system to predict postoperative morbidity and mortality in patients undergoing colorectal surgery ${ }^{29}$. The current study presents another point of view of potentially the same phenomenon, namely physical impairment of the cancer bearing host reflected as functional compromise and systemic inflammation. Cause and effect remain to be determined and in future studies, both SNAQ/GFI/sarcopenia score and GPS should be acquired, for combination of both scores may increase predictive accuracy.

Remarkably, anastomotic leakage and/or intra-abdominal abscess could not be predicted by sarcopenia, SNAQ or GFI scores, nor by their interactions. The predictive factors found in this study were stage 3-4 cancer, i.e. advanced disease and need for blood transfusion. This observation was in line with the results of the large prospective study of Boccola and colleagues ${ }^{30}$. The effect underlying the association between stage 3-4 disease and anastomotic leakage has not been investigated in our study, however a nutritional cause seems unlikely as a high SNAQ score was not independently predictive in multivariate analysis. An increased inflammatory state could be hypothesized in advanced cancer stage, but markers for inflammation were not included in our analyses.

Identifying surgical patients at risk for developing complications remains challenging. Therefore, an important clinical implication can be drawn from this study. The easy-to-perform functional compromise assessment presented here (SNAQ, GFI and sarcopenia) may be used to detect high-risk patients and to adapt treatment regimens accordingly, i.e. primary diverting ileostomy or colostomy. The preoperative period can be considered to improve functional parameters by nutritional support and physical exercise ${ }^{31}$. Exercise may increase physical functioning and overall quality of life in cancer patients ${ }^{32,33}$. The best anabolic response is obtained when exercise is combined with nutritional support, such as essential amino acid ingestion ${ }^{34,35}$. Physical exercise must be performed 2-3 days a week to increase muscle strength ${ }^{36}$, therefore this should be supervised. 
Furthermore, many patients undergo neoadjuvant treatment which generates a timeframe for prehabilitation strategies. Finally, as the SNAQ/GFI/sarcopenia score specifically predicts sepsis, intensive monitoring can be opted for in patients at risk. Future studies may elucidate whether these high-risk patients could benefit from selective decontamination of the digestive tract (SDD) ${ }^{37}$.

The L3 muscle index measure is a widely available, objective and precise measurement for peri-operative assessment of lean body mass. In the current study, a very good interobserver variability was demonstrated by two investigators who had not received any specific radiological training. As mentioned before, an important remark should be made regarding the cut-off values of the L3 index to diagnose sarcopenia. As these values are based on obese cancer patients, they might not be applicable to all populations. Sarcopenia incidence by the definition in the present study was $48 \%$, which is a rather high number in colorectal cancer patients who have not been reported to suffer from severe muscle wasting, potentially indicating an underestimated proportion of functional loss in this group. It is therefore highly desirable to define cut-off values based on healthy subjects and different cancer type populations. Nonetheless, the cut-off values described by Prado et al. are based on mortality prediction, which was supported in the current study. Another limitation is the retrospective nature of the study. However, all patients in the study cohort were included in a prospective database (Dutch Surgical Colorectal Audit) and only patients without an available staging CT scan were excluded. As a consequence, the probability of selection bias was negligible. Due to the retrospective nature of the study, DXA scans and other tests for sarcopenia were not available. However, CT-based muscle area measurement is a well-documented and readily obtainable alternative to detect sarcopenia ${ }^{13}$. The findings of the present study should nonetheless be confirmed in a second, prospective cohort including other tests for sarcopenia.

This study shows that functional compromise in colorectal cancer surgery is associated with adverse postoperative outcome. Moreover, assessment of functional compromise by means of a nutritional questionnaire (SNAQ), a frailty questionnaire (GFI), and CT-based sarcopenia measurement (L3 muscle index), i.e. the SNAQ/GFI/sarcopenia score can accurately predict postoperative sepsis. 
Appendix 1: items of Groningen Frailty Indicator (GFI) score ${ }^{16}$

Mobility

Is the patient able to carry out these tasks single handed without any help? (The use of help resources such as walking stick, walking frame, wheelchair, is considered independent)

- 1. Shopping

- 2. Walking around outside (around the house or to the neighbors)

- 3. Dressing and undressing

- 4. Going to the toilet

Physical Fitness

- 5. What mark does the patient give himself/herself for physical fitness? (scale 0 to 10$)$

Vision

- 6. Does the patient experience problems in daily life due to poor vision?

Hearing

- 7. Does the patient experience problems in daily life due to being hard of hearing?

Nourishment

- 8. During the last 6 months has the patient lost a lot of weight unwillingly? (3 $\mathrm{kg}$ in 1 month or $6 \mathrm{~kg}$ in 2 months)

Morbidity

- 9. Does the patient take 4 or more different types of medicine?

Cognition (Perception)

- 10. Does the patient have any complaints about his/her memory or is the patient known to have a dementia syndrome?

Psychosocial

-11. Does the patient sometimes experience emptiness around him/her?

- 12. Does the patient sometimes miss people around him/her?

-13. Does the patient sometimes feel abandoned?

-14. Has the patient recently felt downhearted or sad?

-15. Has the patient recently felt nervous or anxious?

Sum

Scoring:

- Questions 1-4: Independent = 0; dependent = 1

- Question 5: 0-6 = 1; 7-10 = 0

- Questions 6-9: No = 0; yes = 1

- Question 10: No and sometimes = 0; yes = 1

- Questions 11-15: No = 0; sometimes and yes = 1 
Appendix 2: items of Short Nutritional Assessment Questionnaire (SNAQ) score $^{17}$

Item

Score

Did you lose weight unintentionally?

More than $6 \mathrm{~kg}$ in the last 6 months 3

More than $3 \mathrm{~kg}$ in the last month 2

Did you experience a decreased appetite over the last month? $\quad 1$

Did you use supplemental drinks or tube feeding over the last month? 


\section{References}

1. Jaarrapportage 2010: uitkomst van zorg registratie. Dutch Surgical Colorectal Audit, 2010.

2. Polanczyk CA, Marcantonio E, Goldman L, et al. Impact of age on perioperative complications and length of stay in patients undergoing noncardiac surgery. Ann Intern Med 2001;134:637-43.

3. Law WL, Choi HK, Lee YM, et al. The impact of postoperative complications on longterm outcomes following curative resection for colorectal cancer. Ann Surg Oncol 2007;14:2559-66.

4. Tanaka K, Kumamoto T, Nojiri K, et al. Impact of Postoperative Morbidity on Long-Term Survival After Resection for Colorectal Liver Metastases. Ann Surg Oncol 2010.

5. Walker KG, Bell SW, Rickard MJ, et al. Anastomotic leakage is predictive of diminished survival after potentially curative resection for colorectal cancer. Ann Surg 2004;240:255-9.

6. Makary MA, Segev DL, Pronovost PJ, et al. Frailty as a predictor of surgical outcomes in older patients. J Am Coll Surg 2010;210:901-8.

7. Partridge JS, Harari D, Dhesi JK. Frailty in the older surgical patient: a review. Age Ageing 2012;41:142-7.

8. Tan KY, Kawamura YJ, Tokomitsu A, et al. Assessment for frailty is useful for predicting morbidity in elderly patients undergoing colorectal cancer resection whose comorbidities are already optimized. Am J Surg 2012;204:139-43.

9. Fried LP, Hadley EC, Walston JD, et al. From bedside to bench: research agenda for frailty. Sci Aging Knowledge Environ 2005;2005:pe24.

10. Robinson TN, Wu DS, Stiegmann GV, et al. Frailty predicts increased hospital and six-month healthcare cost following colorectal surgery in older adults. Am J Surg 2011;202:511-4.

11. Saxton A, Velanovich V. Preoperative frailty and quality of life as predictors of postoperative complications. Ann Surg 2011;253:1223-9.

12. Baracos VE, Reiman T, Mourtzakis M, et al. Body composition in patients with non-small cell lung cancer: a contemporary view of cancer cachexia with the use of computed tomography image analysis. Am J Clin Nutr 2010;91:1133S-1137S.

13. Prado CM, Lieffers JR, McCargar $L$, et al. Prevalence and clinical implications of sarcopenic obesity in patients with solid tumours of the respiratory and gastrointestinal tracts: a population-based study. Lancet Oncol 2008;9:629-35.

14. Covinsky KE, Martin GE, Beyth RJ, et al. The relationship between clinical assessments of nutritional status and adverse outcomes in older hospitalized medical patients. J Am Geriatr Soc 1999;47:532-8.

15. Mudge AM, Ross $\sqcup$, Young AM, et al. Helping understand nutritional gaps in the elderly (HUNGER): a prospective study of patient factors associated with inadequate nutritional intake in older medical inpatients. Clin Nutr 2011;30:320-5.

16. Steverink N, Slaets JPJ, Schuurmans $\mathrm{H}$, et al. Measuring frailty: developing and testing the GFI (Groningen Frailty Indicator). Gerontologist 2001;41:236. 
17. Kruizenga HM, Seidell JC, de Vet HC, et al. Development and validation of a hospital screening tool for malnutrition: the short nutritional assessment questionnaire (SNAQ). Clin Nutr 2005;24:75-82.

18. Bone RC, Balk RA, Cerra FB, et al. Definitions for sepsis and organ failure and guidelines for the use of innovative therapies in sepsis. The ACCP/SCCM Consensus Conference Committee. American College of Chest Physicians/Society of Critical Care Medicine. Chest 1992;101:1644-55.

19. Dello SA, Lodewick TM, van Dam RM, et al. Sarcopenia negatively affects preoperative total functional liver volume in patients undergoing liver resection. HPB (Oxford) 2013;15:165-9.

20. Fried LP, Tangen CM, Walston J, et al. Frailty in older adults: evidence for a phenotype. J Gerontol A Biol Sci Med Sci 2001;56:M146-56.

21. Deitch EA. Multiple organ failure. Pathophysiology and potential future therapy. Ann Surg 1992;216:117-34.

22. Deitch EA, Kemper AC, Specian RD, et al. A study of the relationship among survival, gut-origin sepsis, and bacterial translocation in a model of systemic inflammation. J Trauma 1992;32:141-7.

23. Swank GM, Deitch EA. Role of the gut in multiple organ failure: bacterial translocation and permeability changes. World J Surg 1996;20:411-7.

24. Derikx JP, Poeze M, van Bijnen AA, et al. Evidence for intestinal and liver epithelial cell injury in the early phase of sepsis. Shock 2007;28:544-8.

25. Puppa MJ, White JP, Sato S, et al. Gut barrier dysfunction in the Apc(Min/+) mouse model of colon cancer cachexia. Biochim Biophys Acta 2011;1812:1601-6.

26. Richards $\mathrm{CH}$, Roxburgh CS, MacMillan MT, et al. The relationships between body composition and the systemic inflammatory response in patients with primary operable colorectal cancer. PLoS One 2012;7:e41883.

27. Moyes LH, Leitch EF, McKee RF, et al. Preoperative systemic inflammation predicts postoperative infectious complications in patients undergoing curative resection for colorectal cancer. Br J Cancer 2009;100:1236-9.

28. Ishizuka M, Nagata $\mathrm{H}$, Takagi $\mathrm{K}$, et al. Inflammation-based prognostic score is a novel predictor of postoperative outcome in patients with colorectal cancer. Ann Surg 2007;246:1047-51.

29. McMillan DC. The systemic inflammation-based Glasgow Prognostic Score: a decade of experience in patients with cancer. Cancer Treat Rev 2013;39:534-40.

30. Boccola MA, Buettner PG, Rozen WM, et al. Risk factors and outcomes for anastomotic leakage in colorectal surgery: a single-institution analysis of 1576 patients. World J Surg 2011;35:186-95.

31. Fearon KC. The 2011 ESPEN Arvid Wretlind lecture: cancer cachexia: the potential impact of translational research on patient-focused outcomes. Clin Nutr 2012;31:57782.

32. Fong DY, Ho JW, Hui BP, et al. Physical activity for cancer survivors: meta-analysis of randomised controlled trials. BMJ 2012;344:e70. 
33. Mishra SI, Scherer RW, Snyder C, et al. Exercise interventions on health-related quality of life for people with cancer during active treatment. Cochrane Database Syst Rev 2012;8:CD008465.

34. Breen L, Phillips SM. Skeletal muscle protein metabolism in the elderly: Interventions to counteract the 'anabolic resistance' of ageing. Nutr Metab (Lond) 2011;8:68.

35. Volpi E, Kobayashi $\mathrm{H}$, Sheffield-Moore $\mathrm{M}$, et al. Essential amino acids are primarily responsible for the amino acid stimulation of muscle protein anabolism in healthy elderly adults. Am J Clin Nutr 2003;78:250-8.

36. Oldervoll LM, Loge JH, Paltiel $\mathrm{H}$, et al. The effect of a physical exercise program in palliative care: A phase II study. J Pain Symptom Manage 2006;31:421-30.

37. Sleijfer DT, Mulder NH, de Vries-Hospers HG, et al. Infection prevention in granulocytopenic patients by selective decontamination of the digestive tract. Eur J Cancer 1980;16:859-69. 

Chapter 5. Sarcopenia is associated with an increased inflammatory response to surgery in colorectal cancer

Kostan W. Reisinger, Joep P.M. Derikx, Jeroen L.A. van Vugt, Maarten F. Von Meyenfeldt, Karel W. Hulsewé, Steven W.M. Olde Damink, Jan H.M.B. Stoot, Martijn Poeze. Submitted 


\begin{abstract}
Introduction

Sarcopenia in gastrointestinal cancer has been associated with poor clinical outcome after surgery. Differences in inflammatory response to surgery between sarcopenic and non-sarcopenic patients have not been investigated, however skeletal muscle wasting in the context of cachexia is associated with a hyperinflammatory state at baseline. Knowledge on this matter can provide new insight in the detrimental effects of sarcopenia on postoperative recovery, possibly leading to novel therapeutic strategies.
\end{abstract}

\title{
Methods
}

Eighty-seven consecutive patients undergoing elective resection of a primary colorectal tumor were enrolled. Sarcopenia was assessed on routine preoperative computed tomography (CT) scans using image analysis by Osirix ${ }^{\circledR}$ by measuring skeletal muscle on the third lumbar vertebra (L3) level. Differences in preand postoperative plasma levels of C-reactive protein (CRP), calprotectin and interleukin-6 (IL-6) between sarcopenic and non-sarcopenic patients were analyzed. Clinical outcome was assessed by HARM scores.

\section{Results}

Fifty-six patients (64\%) were defined as sarcopenic, using L3 index cut off values of $52.4 \mathrm{~cm}^{2} / \mathrm{m}^{2}$ for men and $38.5 \mathrm{~cm}^{2} / \mathrm{m}^{2}$ for women. Calprotectin levels were higher in sarcopenic patients compared with non-sarcopenic patients at postoperative days 3-5; respectively, 478.7 (SD, 280.6) ng/mL and 330.9 (145.6) $\mathrm{ng} / \mathrm{mL}, p=0.01$. CRP levels were higher in sarcopenic patients on postoperative day 5: 200.1 (207.1) mg/L compared to 56.9 (50.1) mg/L, $p=0.03$. IL-6 levels were not different between groups. Sarcopenic patients had higher HARM scores.

\section{Conclusion}

Sarcopenic patients undergoing surgery for colorectal cancer show an increased postoperative inflammatory response. This may be at least part of the explanation for the high incidence of postoperative complications in sarcopenic patients. 


\section{Introduction}

Frailty in cancer patients has been associated with poor clinical outcome after colorectal surgery ${ }^{1-3}$. The syndrome of frailty is a state of increased vulnerability towards stressors, leading to an increased risk of developing adverse health outcomes ${ }^{4}$. The definitions and biological characteristics of frailty are subject to debate, however weight loss and skeletal muscle wasting are well accepted as prominent features of frailty. Indeed, skeletal muscle wasting or sarcopenia is associated with prolonged length of hospital stay and postoperative infections after colorectal surgery and negatively affects cancer outcomes following resection of colorectal liver metastases ${ }^{5,6}$.

The mechanism by which sarcopenia is associated with impaired postoperative outcome is unknown. A hyperinflammatory response to surgery can be postulated. Although differences in inflammatory response to surgery between sarcopenic and non-sarcopenic patients have not been investigated, skeletal muscle wasting in the context of cachexia is associated with a hyperinflammatory state at baseline 7,8 . Increased inflammatory responses may decrease wound healing and lengthen hospital stay. Moreover, in colorectal surgery impaired wound healing of the gut may manifest as anastomotic leakage, involving significant mortality and health care costs.

This study evaluates whether sarcopenia is associated with increased inflammation after resection of colorectal malignancies by measuring inflammatory markers in plasma. To investigate this hypothesis, plasma levels of a conventional inflammatory parameter, C-reactive protein (CRP), a neutrophil activation marker, calprotectin, and the cytokine interleukin-6 (IL-6) were measured. Furthermore, HARM scores were calculated to analyze clinical outcomes.

\section{Methods}

\section{Patients and sample collection}

Patients who underwent colorectal surgery at Maastricht University Medical Center, Maastricht, the Netherlands and Orbis Medical Center, Sittard-Geleen, the Netherlands were eligible for inclusion in this prospective observational study during the period from January 2011 to September 2013. The study was approved by the medical ethical committee of Maastricht University Medical Center and 
conducted according to the revised version of the Declaration of Helsinki (October 2008, Seoul). The principles of Good Clinical Practice were followed during the study. All patients gave written informed consent.

Venous samples of all patients were collected the day before resection of the colorectal malignancy (PreOp), and daily until the fifth postoperative day (POD 1-5). Blood samples were collected in EDTA-containing vacuum tubes, centrifuged in a pre-chilled centrifuge at $4{ }^{\circ} \mathrm{C}$ (3500 rotations per minute, 15 minutes). Plasma was aliquoted and immediately stored at $-80^{\circ} \mathrm{C}$. After completion of study inclusion, samples were batch analyzed to assess the postoperative inflammatory response. The researchers doing the analyses were blinded from outcome, and the clinicians were blinded from plasma values of different markers, except for CRP. The influence of sarcopenia on clinical outcome was assessed by measuring HARM scores, a validated instrument for postoperative recovery after colorectal surgery ${ }^{9}$. The HARM score comprises mortality, length of hospital stay and readmission expressed as a numeric score of $0-10$, with separate scores for elective and emergent surgery.

\section{Image analysis Osirix ${ }^{\circledR}$}

All patients had a contrast enhanced computed tomography (CT)-scan in their routine preoperative assessment either in our hospital or in one of the surrounding university affiliated district general teaching hospitals. For image analysis, CT scans were used that were provided on CD-Rom.

The areas were calculated with the open source software program Osiri ${ }^{\circledR}$. The 32-bit OsiriX ${ }^{\circledR}$ version 3.3 was downloaded from: http://www.osirix-viewer.com. The areas were measured in a semi-automated fashion. The "Grow Region (2D/3D Segmentation)" tool in the "ROI" dropdown menu made it possible to automatically outline skeletal muscles. This modality of OsiriX ${ }^{\circledR}$ is based on the differences in Hounsfield Units (HU) between the muscles, bony structures, and body fat. The threshold range for muscle was set between -30 and 110 Hounsfield Units (HU). If necessary the automatic outlines were hand-adjusted with the "brush" tool to optimize the Region of Interest (ROI). 


\section{Calculations}

Skeletal muscle content was assessed by measuring the cross-sectional areas of the muscles at the level of the third lumbar vertebral landmark (L3). Measurements were performed on the first image where both vertebral spinae were clearly visible and on the following image in cranial direction. The area of the following muscles were measured: psoas, paraspinal muscles, transversus abdominus, external and internal oblique abdominals and the rectus abdominus. The mean of the skeletal area was used for calculation. The measured skeletal muscles areas were then normalized for height as is conventional for other body composition measures, unit of expression of $L 3$ skeletal muscle index is $\mathrm{cm}^{2} / \mathrm{m}^{2}$.

\section{Sarcopenia}

The definition of sarcopenia was based on a previous study in patients with gastrointesinal and respiratory tract cancer. In this study, the L3 muscle index associated with mortality determined by optimal stratification was $52.4 \mathrm{~cm}^{2} / \mathrm{m}^{2}$ for men and $38.5 \mathrm{~cm}^{2} / \mathrm{m}^{2}$ for women ${ }^{10}$. Patients with a $\mathrm{L} 3$ muscle index lower than these values were considered sarcopenic.

\section{CRP, calprotectin and IL-6 measurement}

As a conventional marker of systemic inflammation, CRP plasma levels were determined by an enzyme-linked immunosorbent assay (ELISA) developed at our institute, as described before (lower detection limit: $100 \mathrm{pg} / \mathrm{mL}$ ) ${ }^{11}$.

Calprotectin, a specific marker of neutrophil activation, was measured as neutrophils play an important role in the early phase of wound healing ${ }^{12,13}$. Calprotectin plasma concentration was measured using the commercially available calprotectin ELISA (lower detection limit 46.8 ng/mL, Hycult Biotechnology Uden, the Netherlands).

Elevated IL-6 levels have been implicated in muscle wasting syndromes like cancer cachexia ${ }^{14}$. Moreover, IL-6 is the predominant stimulator of most acutephase proteins ${ }^{15}$. IL-6 levels were measured by ELISA as previously described (lower detection limit: $10 \mathrm{pg} / \mathrm{mL})^{16}$. 


\section{Statistics}

Sample size was calculated based on the known inflammatory response in patients with colorectal cancer ${ }^{17}$. Of patients with low skeletal muscle mass, $29 \%$ had a Glasgow Prognostic Score (which combines serum CRP and albumin levels) of 2 compared to $5 \%$ of patients with high skeletal muscle mass. With $\alpha=0.05$ and $1-\beta=0.80$, a total sample size of 84 was calculated.

Normality was tested using Kolmogorov-Smirnov. Student's $t$ test was applied for two group comparison for continuous data, and paired $t$ test was used if applicable. Repeated measures one-way analysis of variance (ANOVA) with Bonferroni post hoc test was applied to compare levels of inflammatory markers on different time points between sarcopenic and non-sarcopenic patients. Dichotomous data were compared using Chi square test. Univariate and multivariate linear regression analysis were used to determine whether HARM scores were predicted by marker levels and/or by sarcopenia, to identify whether inflammation or sarcopenia was the main independent determinant of clinical outcome. All data are expressed as mean and SD. A p-value <0.05 was considered statistically significant. Statistical analyses were performed with Prism 5.0 for Windows (GraphPad Software Inc. San Diego, CA) and SPSS 20.0 for Windows (SPSS Inc. Chicago, IL).

TABLE 1. Patient characteristics

\begin{tabular}{|c|c|c|c|c|c|}
\hline & & Sarcopenia & No sarcopenia & Total & $\mathrm{P}$ \\
\hline \multirow[t]{2}{*}{ Sex } & Male & $41(73 \%)$ & $15(48 \%)$ & $56(64 \%)$ & 0.02 \\
\hline & Female & $15(27 \%)$ & $16(52 \%)$ & 31 (36\%) & \\
\hline Age (years) ${ }^{1}$ & & $66.8(13.0)$ & $63.3(9.4)$ & $65.6(11.9)$ & 0.19 \\
\hline $\mathrm{BMI}\left(\mathrm{kg} / \mathrm{m}^{2}\right)^{1}$ & & $25.0(3.3)$ & $27.6(3.7)$ & $25.9(3.6)$ & 0.001 \\
\hline $\begin{array}{l}\text { Duration of surgery } \\
\text { (minutes) }{ }^{1}\end{array}$ & & $130(67)$ & $147(73)$ & $136(69)$ & 0.33 \\
\hline $\begin{array}{l}\text { Peroperative blood loss } \\
(\mathrm{mL})^{1}\end{array}$ & & $289(290)$ & $174(146)$ & $247(253)$ & 0.08 \\
\hline 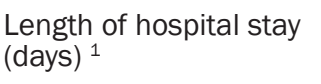 & & $9(7)$ & $6(6)$ & $8(6)$ & 0.09 \\
\hline Laparoscopy & & 22 (39\%) & $14(45 \%)$ & 36 (41\%) & 0.59 \\
\hline \multirow[t]{2}{*}{ Tumor location } & Colon & $27(48 \%)$ & $15(48 \%)$ & $42(48 \%)$ & 0.99 \\
\hline & Rectum & $29(52 \%)$ & $16(52 \%)$ & $45(52 \%)$ & \\
\hline HARM score $^{1}$ & & $3.9(2.1)$ & $2.6(1.9)$ & $3.4(2.1)$ & 0.007 \\
\hline Anastomotic leakage & & $10(19 \%)$ & $1(3 \%)$ & $11(13 \%)$ & 0.04 \\
\hline
\end{tabular}

${ }^{1}$ Data presented as mean (SD) 


\section{Results}

\section{Patients}

Eighty-seven patients were enrolled, of whom 56 were males. Fifty-six patients (64\%) were defined as sarcopenic, using the predefined cut off values.

Forty-one out of 56 male subjects were sarcopenic $(73 \%)$ and 15 out of 31 women (48\%) were sarcopenic. Mean age was 65.6 (SD, 11.9) years. Mean L3 index was $50.5(7.6) \mathrm{cm}^{2} / \mathrm{m}^{2}$ in men and $39.7(5.2) \mathrm{cm}^{2} / \mathrm{m}^{2}$ in women. General characteristics of sarcopenic and non-sarcopenic patients are listed in Table 1. Sarcopenia was predominantly present in men, and sarcopenic patients had a lower BMI compared with non-sarcopenic patients. No other differences in demographic parameters between sarcopenic and non-sarcopenic patients were observed.

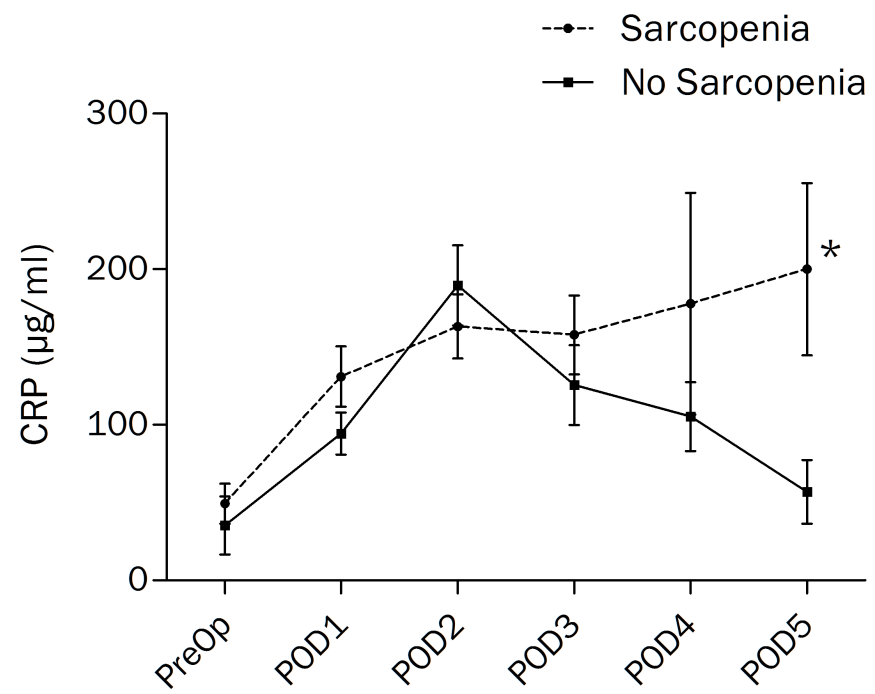

FIGURE 1. Mean plasma levels of CRP. PreOp: preoperative sample. POD: postoperative day. * $p<0.05$. 


\section{CRP}

Plasma CRP levels were significantly higher in sarcopenic compared to nonsarcopenic patients on the fifth postoperative day (POD): 200.1 (207.1) mg/L compared to 56.9 (50.1) $\mathrm{mg} / \mathrm{L}$, respectively, $p=0.03$ (Figure 1). Average postoperative (POD 1-5) plasma CRP levels were not statistically different between sarcopenic and non-sarcopenic patients $(p=0.20)$.

\section{Calprotectin}

Plasma calprotectin levels were comparable between groups at baseline (sarcopenic patients, 174.4 (110.8) ng/mL; non-sarcopenic patients, 140.9 (94.7) $\mathrm{ng} / \mathrm{mL} ; p=0.27$ ), and reached peak values on POD 2 in the non-sarcopenic group, while increasing further on POD 3 in sarcopenic patients (Figure 2). Calprotectin levels at POD 3 were significantly higher in sarcopenic patients (478.7 (280.6) ng/ $\mathrm{mL}$ ) compared with non-sarcopenic patients (330.9 (145.6) ng/mL, $p=0.03)$. This

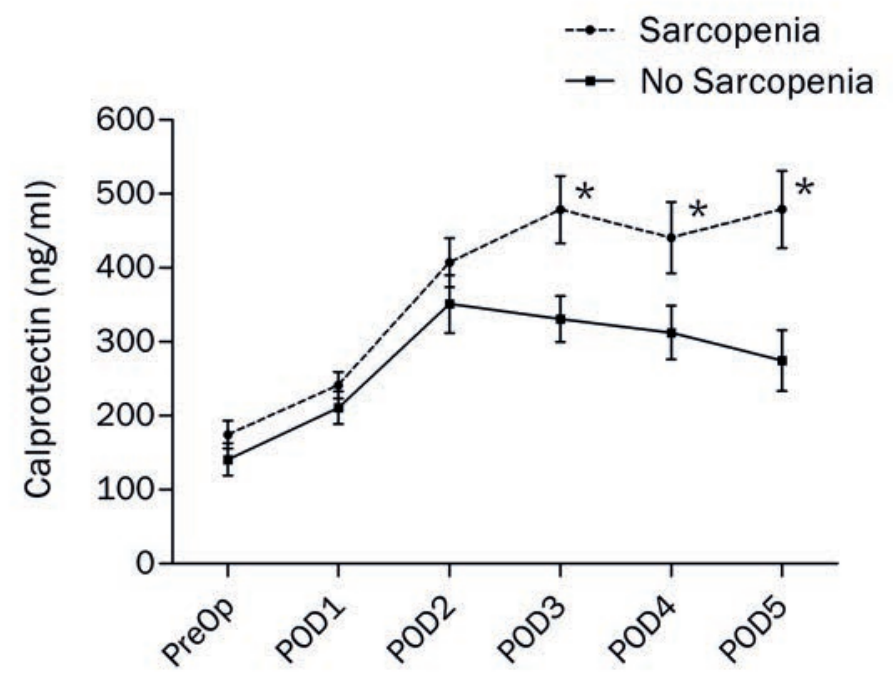

FIGURE 2. Mean plasma levels of calprotectin. PreOp: preoperative sample. POD: postoperative day. * $p<0.05$. 
difference remained present until POD 5. Average postoperative (POD 1-5) values of plasma calprotectin were higher in sarcopenic patients (390.9 $(188.9) \mathrm{ng} / \mathrm{mL}$ ) compared to non-sarcopenic patients (293.8 (120.9) ng/mL, $p=0.02)$.

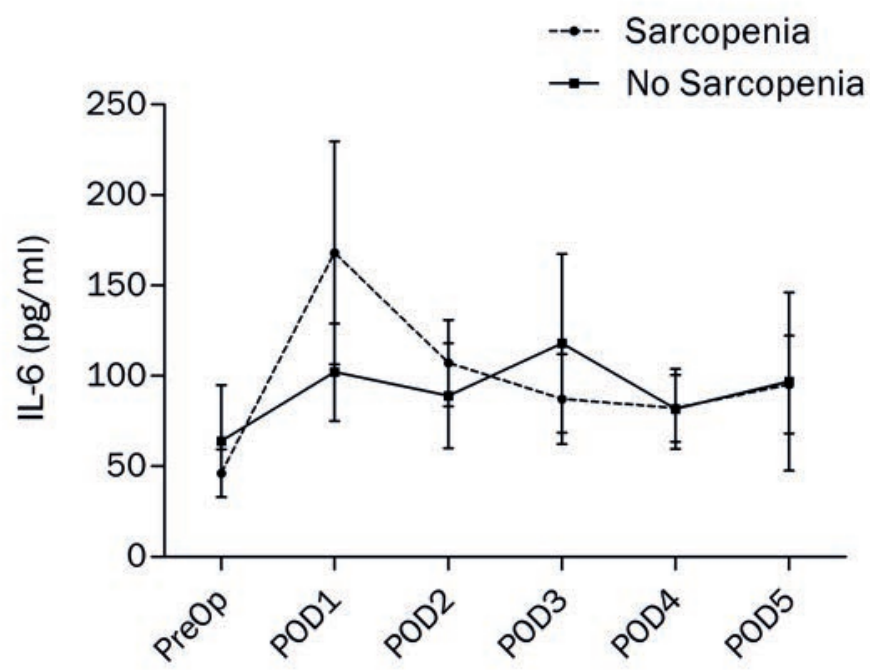

FIGURE 3. Mean plasma levels of IL-6. PreOp: preoperative sample. POD: postoperative day.

\section{IL-6}

IL-6 levels were not significantly different between groups at any time point, however some sarcopenic patients showed remarkably high IL-6 levels at POD1 as reflected by the wide range (Figure 3). Average postoperative (POD 1-5) plasma IL-6 levels were not statistically different between sarcopenic and non-sarcopenic patients $(p=0.54)$.

\section{HARM score}

HARM scores were significantly higher in sarcopenic patients (3.9 (2.1)) compared to non-sarcopenic patients (2.6 (1.9), $p=0.007)$. Furthermore, a higher incidence of anastomotic leakage was observed in the sarcopenic group (19\% versus $3 \%$, $p=0.04$ ) (Table 1). 
In univariate linear regression analysis, sarcopenia $(\beta=0.29, p=0.007)$, average postoperative CRP $(\beta=0.38, \quad p=0.001)$, average postoperative calprotectin $(\beta=0.25, p=0.03)$, and average postoperative IL-6 $(\beta=0.31, p=0.008)$ were all predictive of increased HARM score. In backward stepwise linear regression, the best fit model comprised sarcopenia $(\beta=0.23, p=0.04)$, and average postoperative CRP ( $\beta=0.39, p=0.001)$. This indicates that sarcopenia and average CRP were independently predictive of clinical outcome and that average calprotectin was not independently predictive.

\section{Discussion}

This study shows an increased inflammatory response in sarcopenic patients after colorectal surgery as measured by plasma levels of CRP and calprotectin. Calprotectin is a heterodimeric peptide (36 kDa) constituting $60 \%$ of the cytosolic content of neutrophils and is released from neutrophils upon activation, which indicates importance in wound/suture healing ${ }^{13}$. The current results suggest that activation of this cell type is affected in sarcopenic patients, which may be indicative of an inadequate reaction to surgery. CRP levels reflecting the systemic inflammatory response were elevated in sarcopenic patients a few days after differences in calprotectin levels were observed. This time-frame of postoperative plasma levels of CRP and calprotectin is in concordance with a previous study ${ }^{18}$, indicating that calprotectin may be an early marker of increased postoperative inflammation. The clinical relevance of this finding is indicated by the increased HARM score and anastomotic leakage incidence in sarcopenic patients. Intriguingly, the effect of sarcopenia on clinical outcome appeared to be mediated through increased calprotectin levels in multivariate regression, since only sarcopenia was independently predictive of HARM scores. Of note, average postoperative CRP levels were also independently predictive of HARM scores, however CRP levels were used to guide clinical decisions such as discharge from the hospital. Therefore, CRP levels cannot be considered as an independent predictor.

Although a link between sarcopenia and unfavorable treatment outcomes has been established ${ }^{5,19}$, the mechanism by which sarcopenic patients are predisposed to adverse events is not clear. Sarcopenia, weight loss and cachexia have been linked to increased baseline inflammation ${ }^{20-22}$. However, an increased inflammatory response following surgery has not yet been investigated. After colorectal surgery, prolonged elevation of inflammatory markers is associated with 
severe complications such as anastomotic leakage and sepsis ${ }^{23,24}$, which provides an interesting relationship between sarcopenia and development of complications. Indeed, low muscle mass is associated with postoperative infective complications after colorectal surgery as recently found by Lieffers et al. ${ }^{5}$. The current study underlines the fact that sarcopenic patients represent a distinct population in which caution should be taken, for sarcopenic patients displayed a higher HARM score and anastomotic leakage incidence. This finding was underlined in a recent cohort of 284 patients who underwent oncologic colorectal surgery between January 2010 and May 2012 in Orbis Medical Center. In this cohort, HARM scores were also significantly higher in sarcopenic patients compared to non-sarcopenic patients (data not shown). Additional measures in sarcopenic patients could include preoperative optimization, i.e. strategies to improve muscle quality and quantity. For example, Ryan et al. showed that perioperative supplementation using enteral nutrition enriched with eicosapentaenoic acid (EPA) improved lean body mass in esophageal surgery ${ }^{25}$. Yuill and colleagues showed that supplementation of oral carbohydrates attenuated postoperative skeletal muscle mass depletion in major upper gastrointestinal surgery ${ }^{26}$. Future studies should address the question whether sarcopenic colorectal cancer patients may benefit from such approach in terms of functional recovery, disease recurrence and disease-free survival.

This observational study was designed to detect differences in plasma markers of inflammation and was thus not powered to elicit any effects on clinical outcome. Therefore the results should be interpreted with caution. Although the data are highly suggestive, it remains to be determined whether the increased inflammatory response in sarcopenic patients is indeed a pathological effect associated with adverse outcome. In other studies, a high neutrophil to lymphocyte ratio (NLR) was associated with impaired short-term and long-term outcome in patients undergoing colorectal surgery ${ }^{27}$, indicating that increased neutrophil activation measured by calprotectin levels may mirror this phenomenon. Another limitation of the study is that the origin of increased neutrophil activation could not be determined with the current design. In the early phase of wound healing, neutrophils are activated and recruited to the wound bed. Intestinal inflammation is characterized by influx of neutrophils, however neutrophil activation products could have been produced at the surgical wound site as well. This would be interesting to know, as exaggerated intestinal inflammation may lead to inadequate anastomotic healing and translocation of bacterial products. 


\section{References}

1. Tan KY, Kawamura YJ, Tokomitsu A, et al. Assessment for frailty is useful for predicting morbidity in elderly patients undergoing colorectal cancer resection whose comorbidities are already optimized. American journal of surgery 2012;204:139-43.

2. Robinson TN, Wu DS, Stiegmann GV, et al. Frailty predicts increased hospital and six-month healthcare cost following colorectal surgery in older adults. Am J Surg 2011;202:511-4.

3. Reisinger KW, van Vugt JL, Tegels JJ, et al. Functional Compromise Reflected by Sarcopenia, Frailty, and Nutritional Depletion Predicts Adverse Postoperative Outcome After Colorectal Cancer Surgery. Ann Surg 2014.

4. Fried LP, Hadley EC, Walston JD, et al. From bedside to bench: research agenda for frailty. Sci Aging Knowledge Environ 2005;2005:pe24.

5. Lieffers JR, Bathe OF, Fassbender K, et al. Sarcopenia is associated with postoperative infection and delayed recovery from colorectal cancer resection surgery. Br J Cancer 2012;107:931-6.

6. van Vledder MG, Levolger S, Ayez N, et al. Body composition and outcome in patients undergoing resection of colorectal liver metastases. Br J Surg 2012;99:550-7.

7. Muscaritoli M, Anker SD, Argiles J, et al. Consensus definition of sarcopenia, cachexia and pre-cachexia: joint document elaborated by Special Interest Groups (SIG) "cachexia-anorexia in chronic wasting diseases" and "nutrition in geriatrics". Clin Nutr 2010;29:154-9.

8. Scheede-Bergdahl C, Watt HL, Trutschnigg B, et al. Is IL-6 the best pro-inflammatory biomarker of clinical outcomes of cancer cachexia? Clin Nutr 2012;31:85-8.

9. Keller DS, Chien HL, Hashemi L, et al. The HARM Score: A Novel, Easy Measure to Evaluate Quality and Outcomes in Colorectal Surgery. Ann Surg 2013.

10. Prado CM, Lieffers JR, McCargar $\sqcup$, et al. Prevalence and clinical implications of sarcopenic obesity in patients with solid tumours of the respiratory and gastrointestinal tracts: a population-based study. Lancet Oncol 2008;9:629-35.

11. van Dielen FM, van't Veer C, Schols AM, et al. Increased leptin concentrations correlate with increased concentrations of inflammatory markers in morbidly obese individuals. Int J Obes Relat Metab Disord 2001;25:1759-66.

12. Steinbakk M, Naess-Andresen CF, Lingaas E, et al. Antimicrobial actions of calcium binding leucocyte L1 protein, calprotectin. Lancet 1990;336:763-5.

13. Fagerhol MK. Calprotectin, a faecal marker of organic gastrointestinal abnormality. Lancet 2000;356:1783-4.

14. Strassmann G, Fong M, Kenney JS, et al. Evidence for the involvement of interleukin 6 in experimental cancer cachexia. J Clin Invest 1992;89:1681-4.

15. Gabay C, Kushner I. Acute-phase proteins and other systemic responses to inflammation. N Engl J Med 1999;340:448-54.

16. Hanssen SJ, Derikx JP, Vermeulen Windsant IC, et al. Visceral injury and systemic inflammation in patients undergoing extracorporeal circulation during aortic surgery. Ann Surg 2008;248:117-25. 
17. Richards $\mathrm{CH}$, Roxburgh CS, MacMillan MT, et al. The relationships between body composition and the systemic inflammatory response in patients with primary operable colorectal cancer. PLoS One 2012;7:e41883.

18. Berger D, Bolke E, Seidelmann M, et al. Time-scale of interleukin-6, myeloid related proteins (MRP), C reactive protein (CRP), and endotoxin plasma levels during the postoperative acute phase reaction. Shock 1997;7:422-6.

19. Windsor JA, Hill GL. Protein depletion and surgical risk. Aust N Z J Surg 1988;58:711-5.

20. Kim JK, Choi SR, Choi MJ, et al. Prevalence of and factors associated with sarcopenia in elderly patients with end-stage renal disease. Clin Nutr 2013.

21. Deans DA, Tan BH, Wigmore SJ, et al. The influence of systemic inflammation, dietary intake and stage of disease on rate of weight loss in patients with gastro-oesophageal cancer. Br J Cancer 2009;100:63-9.

22. Cruz-Jentoft AJ, Baeyens JP, Bauer JM, et al. Sarcopenia: European consensus on definition and diagnosis: Report of the European Working Group on Sarcopenia in Older People. Age Ageing 2010;39:412-23.

23. Platt JJ, Ramanathan ML, Crosbie RA, et al. C-reactive Protein as a Predictor of Postoperative Infective Complications after Curative Resection in Patients with Colorectal Cancer. Ann Surg Oncol 2012;19:4168-77.

24. Lagoutte N, Facy O, Ravoire A, et al. C-reactive protein and procalcitonin for the early detection of anastomotic leakage after elective colorectal surgery: Pilot study in 100 patients. J Visc Surg 2012;149:e345-9.

25. Ryan AM, Reynolds JV, Healy L, et al. Enteral nutrition enriched with eicosapentaenoic acid (EPA) preserves lean body mass following esophageal cancer surgery: results of a double-blinded randomized controlled trial. Ann Surg 2009;249:355-63.

26. Yuill KA, Richardson RA, Davidson HI, et al. The administration of an oral carbohydratecontaining fluid prior to major elective upper-gastrointestinal surgery preserves skeletal muscle mass postoperatively--a randomised clinical trial. Clin Nutr 2005;24:32-7.

27. Guthrie GJ, Charles KA, Roxburgh CS, et al. The systemic inflammation-based neutrophil-lymphocyte ratio: experience in patients with cancer. Crit Rev Oncol Hematol 2013;88:218-30. 

Chapter 6. Loss of skeletal muscle mass during neoadjuvant therapy predicts postoperative mortality in esophageal cancer surgery

Kostan W. Reisinger, Joanna W.A.M. Bosmans, Martine Uittenbogaart, Abdulaziz Alsoumali, Martijn Poeze, Meindert N. Sosef, Joep P.M. Derikx. Annals of Surgical Oncology. In press 


\begin{abstract}
Background

Surgery for esophageal cancer is associated with serious complications and mortality. It is therefore of crucial importance to develop tools predicting unfavourable postoperative outcome. As esophageal cancer and neoadjuvant chemotherapy both induce skeletal muscle wasting and thereby impaired physiologic reserves, the degree of muscle mass lost during neoadjuvant therapy may predict postoperative mortality.
\end{abstract}

\title{
Method
}

123 consecutive patients undergoing surgery for esophageal malignancy were included. Skeletal muscle mass was measured on routinely performed CT scans by assessing L3 muscle index prior to and after neoadjuvant therapy and the amount of muscle mass lost during neoadjuvant therapy (muscle loss index, MLI) was calculated. It was investigated whether this amount was associated with postoperative 30-day or in-hospital mortality and morbidity.

\section{Results}

In the complete cohort, no significant association between loss of muscle mass and mortality was found. However, skeletal muscle mass was significantly lower in patients with stage III-IV tumors compared to patients with stage I-II tumors. In the stage III-IV subgroup, the amount of muscle mass lost during neoadjuvant therapy was predictive of complication related mortality; $-13.5 \%$ (SD, 6.2\%) in patients who died postoperatively compared to $-5.0 \%$ (SD, 8.3\%) in surviving patients, $p=0.02$. An optimal cut-off point to predict postoperative mortality in this group was found (-13.2\%) with sensitivity, $80 \%$ and specificity, $85 \%, p=0.03$.

\section{Conclusion}

CTbased measurementofmusclemass lossduringneoadjuvantchemoradiotherapy may provide a readily available and inexpensive tool to identify patients at risk for developing unfavorable postoperative outcome after resection of esophageal malignancies, especially in patients with stage III-IV tumors. 


\section{Introduction}

Complications after esophageal resection present a serious problem: $26-41 \%$ of patients develop major adverse events, the majority being cardiopulmonary and infectious complications or anastomotic leakage, and mortality rates are $4-10 \%$ 1. It is therefore imperative to develop tools that adequately predict unfavorable postoperative outcome. Sarcopenia may be such a tool. Sarcopenia is defined as a syndrome characterized by progressive and generalized loss of skeletal muscle mass and strength, which increases the risk of adverse health-related outcomes such as impaired physical ability, decreased quality of life and mortality 2, 3. Neoadjuvant chemoradiotherapy (CRT) induces significant sarcopenia in esophageal cancer patients ${ }^{4}$. Moreover, esophageal cancer can further deteriorate skeletal muscle mass by causing cachexia. Sarcopenia is associated with complications following surgery ${ }^{5,6}$.

A readily available instrument to quantify skeletal muscle mass is CT-based muscle area measurement at the level of the lumbar 3 vertebral landmark, which can be accomplished by using open-source software ${ }^{7,8}$. Although low preoperative muscle mass measured on CT scans could not be correlated with mortality and length of hospital stay in patients undergoing esophageal surgery ${ }^{4}$, the amount of muscle mass loss induced by neoadjuvant therapy may be a more dynamic predictor of postoperative outcome. Increased muscle loss prior to surgery presumably reflects high metabolic stress due to malnutrition, cachexia, tumor load or inflammation, negatively affecting the metabolic response to major gastrointestinal surgery. In this study, it was hypothesized that the amount of muscle mass loss during neoadjuvant therapy predicts complication related mortality after esophageal resection. Therefore, muscle mass was measured prior to and after neoadjuvant treatment in patients undergoing esophageal resection and the association between amount of muscle mass lost and postoperative morbidity and mortality was investigated.

\section{Methods}

\section{Patients}

All patients who underwent resection of esophageal cancer in a single teaching hospital from January 2008 until January 2012 were enrolled in a prospectively maintained digital database. Data included characteristics of the primary tumor and oncologic staging, specifications of surgical treatment, chemotherapy, 
radiotherapy, and postoperative complications. All patients receiving neoadjuvant CRT were included in this study. Neoadjuvant therapy consisted of 3 cycles cisplatin/5-fluorouracil (CF) or 5 cycles paclitaxel/carboplatin (PC), all with concurrent radiotherapy, or consisted of 3 cycles epirubicin/cisplatin/ capecitabine (ECC) in some patients. A routine total body PET/CT scan before before neoadjuvant therapy (preCRT) and after neoadjuvant therapy (postCRT) was performed. Patients were closely monitored by a dietician from the moment of diagnosis until hospital discharge after surgery and malnutrition was screened for. Furthermore, physical activity was stimulated by counseling or by active preconditioning by an expert-physiotherapist.

\section{Complication related mortality}

The primary clinical outcome of the study was postoperative complication related mortality. This was defined as mortality within 30 days or within hospital admission, associated with postoperative complications.

\section{CT-based muscle measurements}

CT-based measurements were performed using Osirix ${ }^{\circledR}$ Version 3.3 (32-bit; http:// www.osirix.com) by a researcher who was blinded from outcome. The crosssectional skeletal muscle surface $\left(\mathrm{cm}^{2}\right)$ as measure of sarcopenia was measured at the level of the third lumbar vertebra (L3) on two consecutive transversal coupes on which both vertebral spines were visible ${ }^{8}$. The 'Grow Region (2D/3D Segmentation)' tool in the menu of the program facilitated to automatically select all skeletal muscle mass in one coupe. The distinction between different tissues was based on Hounsfield Units (HU) with a threshold range of $-30 \mathrm{HU}$ to $+110 \mathrm{HU}$ used for skeletal muscle. Muscles measured were: psoas, paraspinal, transverse abdominal, external oblique, internal oblique and rectus abdominis muscles. Hand-adjustment of the selected areas was performed if necessary and the muscle area was calculated automatically ${ }^{8}$.

\section{Sarcopenia and muscle loss index (MLI)}

The L3 muscle area surfaces were normalized for patient height to calculate the L3 muscle index and expressed in $\mathrm{cm}^{2} / \mathrm{m}^{2}$. The cut-off values used for sarcopenia were $52.4 \mathrm{~cm}^{2} / \mathrm{m}^{2}$ for men and $38.5 \mathrm{~cm}^{2} / \mathrm{m}^{2}$ for women, based on the method 
of Prado et al. " . The muscle loss index (MLI), as a measure of muscle mass loss during chemoradiotherapy, was calculated as follows: (postCRT L3 index - preCRT L3 index) / preCRT L3 index * 100\%.

TABLE 1. Patient, tumor, and operation characteristics

\begin{tabular}{|c|c|c|c|}
\hline & & Number of patients & Mean (SD) \\
\hline \multirow[t]{2}{*}{ Sex } & Male & $101(82.1 \%)$ & \\
\hline & Female & $22(17.9 \%)$ & \\
\hline \multirow[t]{2}{*}{ Age (years) } & & & $63(10)$ \\
\hline & $>70$ & 32 (26\%) & \\
\hline \multirow[t]{2}{*}{ BMI (kg/m²) } & & & $24.7(4.2)$ \\
\hline & $>30$ & $13(10.6 \%)$ & \\
\hline $\begin{array}{l}\text { Length of hospital stay } \\
\text { (days) }\end{array}$ & & & $26(33)$ \\
\hline \multirow[t]{3}{*}{ ASA } & 1 & $30(25.4 \%)$ & \\
\hline & II & 52 (44.1\%) & \\
\hline & III & $36(30.5 \%)$ & \\
\hline \multirow[t]{2}{*}{ Stage } & $|-| \mid$ & $61(49.6 \%)$ & \\
\hline & III-IV & $62(50.4 \%)$ & \\
\hline \multicolumn{2}{|c|}{$\begin{array}{l}\text { Time between preCRT CT } \\
\text { and postCRT CT (days) }\end{array}$} & & $111(17)$ \\
\hline \multicolumn{2}{|l|}{$\begin{array}{l}\text { Time between postCRT CT } \\
\text { and operation (days) }\end{array}$} & & $33(19)$ \\
\hline \multirow[t]{3}{*}{ Type of chemotherapy } & CF & $108(87.8 \%)$ & \\
\hline & ECC & $6(4.9 \%)$ & \\
\hline & PC & $9(7.3 \%)$ & \\
\hline \multirow[t]{2}{*}{ Tumor type } & Adenocarcinoma & 100 (81.3\%) & \\
\hline & Squamous cell carcinoma & $23(18.7 \%)$ & \\
\hline \multirow[t]{5}{*}{ Tumor location } & Proximal esophageal & $1(0.9 \%)$ & \\
\hline & Mid esophageal & 20 (17.9\%) & \\
\hline & Distal esophageal & 57 (48.7\%) & \\
\hline & Juctional & $29(24.8 \%)$ & \\
\hline & Gastric & $10(8.5 \%)$ & \\
\hline \multirow[t]{2}{*}{ Type of surgery } & Transthoracic & $58(47.2 \%)$ & \\
\hline & Transhiatal & $65(52.8 \%)$ & \\
\hline Blood loss (mL) & & & $664(970)$ \\
\hline
\end{tabular}

ASA: American Society of Anesthesiologists; CRT: chemoradiotherapy; CF: cisplatin/5fluorouracil ECC: epirubicin/cisplatin/capecitabine; PC: paclitaxel/carboplatin 


\section{Statistical analysis}

Normality was tested using Kolmogorov-Smirnov. Frequencies are presented as absolute numbers and percentages. Continuous data are presented as mean and standard deviation (SD). Differences between groups were analyzed with the Pearson chi-square test for dichotomous parameters. Receiver operating characteristic (ROC) curves were used to calculate accuracy of the relative L3 index decrease to predict complication related mortality. The ideal cut-off value for predicting mortality was defined as the cut-off value with maximum sum of sensitivity and specificity. Overall diagnostic accuracy was represented by the area under the curve (AUC). Two-tailed p-values <0.05 were considered significant. All statistical analyses were performed using SPSS ${ }^{\circledR} 20.0$ (SPSS Inc, Chicago, IL, USA).

\section{Results}

\section{Patients and L3 index during chemoradiotherapy}

123 patients were included, of whom 101 (82\%) were males. Patient, tumor and operation characteristics are listed in Table 1. Both a preCRT and postCRT CT scan were available in 96 patients. Sarcopenia was present in 60/123 patients (49\%) before CRT and in 74/123 patients (60\%) after CRT. Concomitantly, the mean L3-index decreased significantly during CRT $\left(50.9\right.$ (SD, 8.5) $\mathrm{cm}^{2} / \mathrm{m}^{2}$ to 48.4 (8.5) $\mathrm{cm}^{2} / \mathrm{m}^{2}, p<0.001$, Fig $\left.1 \mathrm{~A}\right)$. Mortality within 30 days or within hospital admission was $11 / 123(9 \%)$ in the total cohort and 6/62 (10\%) in patients with stage III-IV tumors.
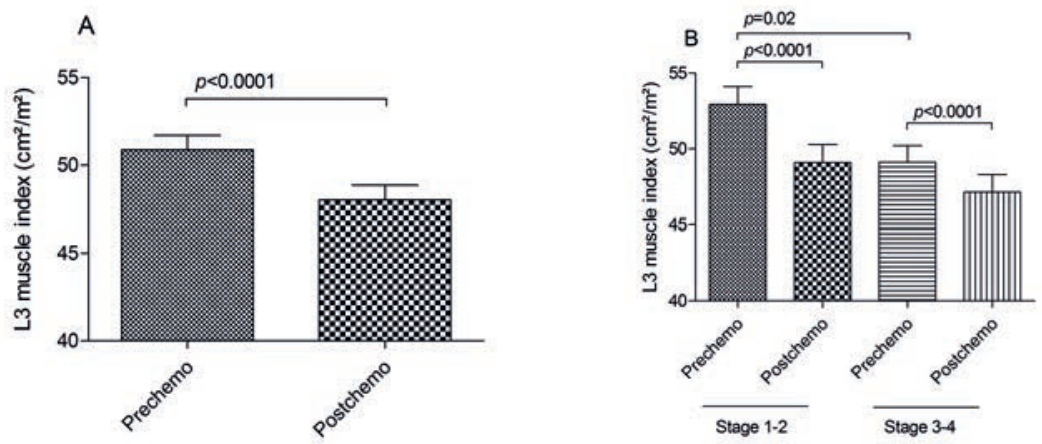

FIGURE 1. L3 muscle index before and after neoadjuvant treatment in patients undergoing esophageal surgery. A. Complete cohort. B. By tumor stage (stage 3-4 versus 1-2). 


\section{Influence of tumor characteristics on the $L 3$ index}

The mean L3 index was significantly lower before CRT began in patients with advanced (stage III-IV) tumors compared with localized tumors $\left(49.1(8.4) \mathrm{cm}^{2}\right.$ / $\mathrm{m}^{2}$ and $\left.52.9(8.4) \mathrm{cm}^{2} / \mathrm{m}^{2}, p=0.02\right)$, Fig 1B. In both stage III-IV and stage I-II tumors, the L3 index decreased significantly during CRT. L3 indices were not different between patients with adenocarcinoma and patients with squamous cell carcinoma.
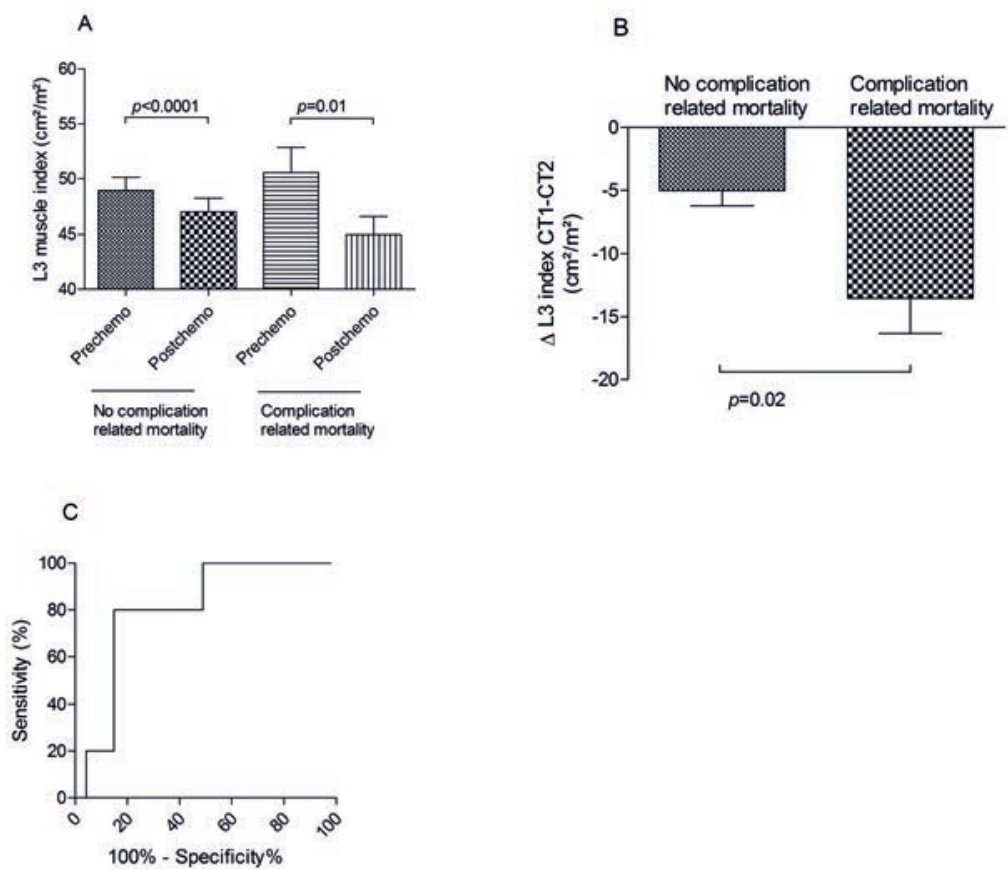

FIGURE 2. Loss of muscle mass measured as decrease of L3 muscle index in patients with advanced (stage 3-4) tumors. A. L3 indices before and after chemoradiotherapy in surviving patients and patients who die from postoperative complications. B. Relative L3 index decrease in surviving patients and patients who die from postoperative complications. C. ROC curve of relative L3 index decrease to predict postoperative complication related mortality. 


\section{L3 index decrease as a predictor of mortality}

The MLI was not predictive for postoperative mortality directly related to complications in the total study population; -8.6\% (7.6\%) in patients who died postoperatively compared with $-7.0 \%(8.0 \%)(p=0.57)$ in surviving patients. As L3 indices differed significantly between stage III-IV and stage I-II disease, these groups were analyzed separately. In patients with stage I-II tumors, MLI was not different between patients with and those without complication related mortality; respectively, $-2.5 \%(3.6 \%)$ and $-9.4 \%$ (7.1\%), $p=0.07$. In patients with stage III-IV tumors, patients who died postoperatively showed significantly higher decrease in $\mathrm{MLI}(-13.5 \%(6.2 \%))$ compared to surviving patients $(-5.0 \%(8.3 \%), p=0.02)$, Fig $2 \mathrm{~A}, \mathrm{~B}$.

To determine diagnostic accuracy of the MLI to detect postoperative complication related mortality in patients with stage III-IV disease, ROC curve analysis was done (Fig 2C). An optimal cut-off point of $-13.2 \%$ was found with sensitivity, $80 \%$; specificity, 85\%; positive likelihood ratio, 5.33 (95\% confidence interval (Cl), 2.5 11.0); negative likelihood ratio, $0.24(95 \% \mathrm{Cl}, 0.04-1.4) ; p=0.03$. The area under the ROC curve was 0.80 (95\% $\mathrm{Cl}, 0.64-0.96)$.

\section{Discussion}

This study confirms the loss of muscle mass during neoadjuvant CRT in patients who undergo resection of esophageal malignancies. It is shown for the first time that loss of muscle mass, as measured on routinely obtained CT scans, correlates well with adverse outcome in patients with advanced tumors, i.e. in patients with lowered preCRT muscle mass. This may provide a readily available and inexpensive tool to identify patients at risk for developing unfavorable postoperative outcome.

The application of patient-tailored approaches is upcoming in oncologic health care ${ }^{9}$. In this context, measurements of the physical ability to adequately recover from therapeutic hits such as surgery is vital, particularly in the tumor-bearing patient in whom metabolic and nutritional resources are generally depleted. Considering the striking impact of metastasized disease on physiological depletion, patients with stage III and IV tumors are more prone to loss of reserves, manifesting as cachexia which includes loss of muscle mass. The current data reflect this effect by lower L3 muscle index in patients with stage III-IV disease, similar to Ravasco et al., who found elevated resting energy expenditure in patients with stage III-IV colorectal tumors ${ }^{10}$. Selection of patients with increased risk for 
poor postoperative outcome within this vulnerable group is therefore important. Delaying the moment of surgery and with application of prehabilitation programs to regain physical resources or preventive postoperative ICU admissions may offer advantages in patients who are identified as having high risk for poor outcome.

The inability of a hospital to let patients with severe complications survive is known as failure to rescue (FTR). Instead of higher complication rates, higher FTR rates are the main determinant of postoperative mortality ${ }^{11}$. FTR variability between hospitals is largely contributed to hospital related factors, such as the available level of ICU care ${ }^{12}$. On the other hand, variability of patient characteristics influences FTR rates as well ${ }^{13}$. The amount of muscle mass lost during neoadjuvant therapy could be used as a predictor of FTR rates in future studies.

Our data are in line with several studies. Deans and colleagues showed that weight loss, among others, was independently predictive of death after esophageal resections ${ }^{14}$. This study, however, focused on long-term mortality (disease prognosis), therefore weight loss may actually be a reflection of tumor progression rather than functional depletion. In their recent study, Awad et al. showed a decrease in muscle mass during CRT as measured on the L3 level on CT scans ${ }^{4}$, although a correlation with adverse postoperative outcome could not be found. Patients with advanced tumors were not analyzed separately from those with local tumors, as in the current study.

As with every observational study, the current data should be taken with caution. Although the studied cohort was relatively large in its kind, numbers of complication related deaths are small. Therefore, the observed effect should be validated in a larger, preferably multicentre or nationwide cohort. Moreover, it should be addressed whether attempts to attenuate loss of muscle mass during neoadjuvant therapy are effective and whether they result in decreased FTR rates. In the studied population, efforts to counteract exhaustion of physiologic reserves by nutritional support were already done, indicating that nutrition alone is not the key to success. Several systematic reviews and meta-analyses conclude that physical exercise may improve physical functioning and overall quality of life in cancer patients ${ }^{15,16}$. Strength training is the most effective exercise to slow down the rate of loss of muscle mass and maintaining and improving muscle strength. The combination of physical exercise with essential amino acid ingestion elicits the greatest anabolic response ${ }^{17,18}$. As physical exercise is only effective in terms of improving muscle strength when performed 2-3 days a week ${ }^{19}$, the feasibility of this approach in combination with the physical and mental side-effects of 
neoadjuvant therapy should be determined. Finally, eicosapentaenoic acid (EPA) can preserve lean body mass after esophageal surgery, and may therefore be an interesting option to preserve muscle mass during CRT ${ }^{20}$. 


\section{References}

1. Enzinger PC, Mayer RJ. Esophageal cancer. N Engl J Med 2003;349:2241-52.

2. Delmonico MJ, Harris TB, Lee JS, et al. Alternative definitions of sarcopenia, lower extremity performance, and functional impairment with aging in older men and women. J Am Geriatr Soc 2007;55:769-74.

3. Goodpaster BH, Park SW, Harris TB, et al. The loss of skeletal muscle strength, mass, and quality in older adults: the health, aging and body composition study. J Gerontol A Biol Sci Med Sci 2006;61:1059-64.

4. Awad S, Tan BH, Cui H, et al. Marked changes in body composition following neoadjuvant chemotherapy for oesophagogastric cancer. Clin Nutr 2012;31:74-7.

5. Lieffers JR, Bathe OF, Fassbender K, et al. Sarcopenia is associated with postoperative infection and delayed recovery from colorectal cancer resection surgery. Br J Cancer 2012;107:931-6.

6. van Vledder MG, Levolger S, Ayez N, et al. Body composition and outcome in patients undergoing resection of colorectal liver metastases. Br J Surg 2012;99:550-7.

7. Prado CM, Lieffers JR, McCargar $\sqcup$, et al. Prevalence and clinical implications of sarcopenic obesity in patients with solid tumours of the respiratory and gastrointestinal tracts: a population-based study. Lancet Oncol 2008;9:629-35.

8. Dello SA, Lodewick TM, van Dam RM, et al. Sarcopenia negatively affects preoperative total functional liver volume in patients undergoing liver resection. HPB (Oxford) 2013;15:165-9.

9. Mendelsohn J. Personalizing oncology: perspectives and prospects. J Clin Oncol 2013;31:1904-11.

10. Ravasco P, Monteiro-Grillo I, Camilo M. Colorectal cancer: intrinsic characteristics modulate cancer energy expenditure and the risk of cachexia. Cancer Invest 2007;25:308-14.

11. Henneman D, Snijders HS, Fiocco M, et al. Hospital variation in failure to rescue after colorectal cancer surgery: results of the Dutch Surgical Colorectal Audit. Ann Surg Oncol 2013;20:2117-23.

12. Henneman D, van Leersum NJ, Ten Berge M, et al. Failure-to-Rescue After Colorectal Cancer Surgery and the Association with Three Structural Hospital Factors. Ann Surg Oncol 2013;20:3370-6.

13. Friese CR, Earle CC, Silber JH, et al. Hospital characteristics, clinical severity, and outcomes for surgical oncology patients. Surgery 2010;147:602-9.

14. Deans DA, Wigmore SJ, de Beaux AC, et al. Clinical prognostic scoring system to aid decision-making in gastro-oesophageal cancer. Br J Surg 2007;94:1501-8.

15. Fong DY, Ho JW, Hui BP, et al. Physical activity for cancer survivors: meta-analysis of randomised controlled trials. BMJ 2012;344:e70.

16. Mishra SI, Scherer RW, Snyder C, et al. Exercise interventions on health-related quality of life for people with cancer during active treatment. Cochrane Database Syst Rev 2012;8:CD008465. 
17. Breen L, Phillips SM. Skeletal muscle protein metabolism in the elderly: Interventions to counteract the 'anabolic resistance' of ageing. Nutr Metab (Lond) 2011;8:68.

18. Volpi E, Kobayashi $\mathrm{H}$, Sheffield-Moore $\mathrm{M}$, et al. Essential amino acids are primarily responsible for the amino acid stimulation of muscle protein anabolism in healthy elderly adults. Am J Clin Nutr 2003;78:250-8.

19. Oldervoll LM, Loge JH, Paltiel H, et al. The effect of a physical exercise program in palliative care: A phase II study. J Pain Symptom Manage 2006;31:421-30.

20. Ryan AM, Reynolds JV, Healy L, et al. Enteral nutrition enriched with eicosapentaenoic acid (EPA) preserves lean body mass following esophageal cancer surgery: results of a double-blinded randomized controlled trial. Ann Surg 2009;249:355-63. 




\section{Part 2 \\ Biomarkers of complications after adult gastrointestinal surgery}

The second aim was to investigate biomarkers to detect complications early after colorectal surgery. Next to the need for developing knowledge on causal factors of postoperative complications and strategies to prevent them, it is also of crucial importance to diagnose major complications at an early time point. Early and accurate recognition of postoperative complications will facilitate timely and appropriate clinical decisions and may thereby lead to less severe symptoms and decreased mortality. One of the most feared complications after colorectal surgery is anastomotic leakage, which is characterized by failure of intestinal wound healing, excessive inflammation, leakage of intestinal luminal contents into the abdominal cavity, peritonitis, sepsis and ultimately death if not treated adequately. In chapter 7 , several plasma biomarkers reflecting intestinal cell damage and inflammation were investigated as possible diagnostic tools to detect anastomotic leakage. 

Chapter 7. Accurate prediction of anastomotic leakage after colorectal surgery using plasma markers for intestinal damage and inflammation

Kostan W. Reisinger, Martijn Poeze, Karel W.E. Hulsewé, Bernadette A. van Acker, Annemarie A. van Bijnen, Anton G.M. Hoofwijk, Jan H. Stoot, Joep P.M. Derikx. Journal of the American College of Surgeons 2014. 219(4):744-51 


\begin{abstract}
Background

Anastomotic leakage is a frequent and life-threatening complication after colorectal surgery. Early recognition of anastomotic leakage is critical to reduce mortality. As early clinical and radiological signs of anastomotic leakage are often non-specific, there is an urgent need for accurate biomarkers. Markers of inflammation and gut damage may be suitable, as these are hallmarks of anastomotic leakage.
\end{abstract}

\title{
Study design
}

In 84 patients undergoing scheduled colorectal surgery with primary anastomosis, plasma samples were collected preoperatively, and daily after surgery. Inflammatory markers C-reactive protein (CRP), calprotectin, and IL-6; and intestinal damage markers intestinal fatty acid binding protein (I-FABP), liver fatty acid binding protein (L-FABP), and ileal bile acid binding protein (I-BABP) were measured. Diagnostic accuracy of single markers or combinations of markers was analyzed by ROC curve analysis.

\section{Results}

Eight patients developed anastomotic leakage, clinically diagnosed at median day 6. Calprotectin had best diagnostic accuracy to detect anastomotic leakage postoperatively. Highest diagnostic accuracy was obtained when CRP and calprotectin were combined at postoperative day (POD) 3, yielding sensitivity, 100\%; specificity, 89\%; LR+, 9.09 (95\% Cl, 4.34 - 16); and LR-, 0.00 (95\% Cl, 0.00 - 0.89); $p<0.001$. Interestingly, preoperative I-FABP levels predicted AL at a cut-off level of $882 \mathrm{pg} / \mathrm{mL}$ with sensitivity, 50\%; specificity, 100\%; LR+, infinite (95\% Cl, 4.01 - infinite); LR-, 0.50 (95\% Cl, 0.26 - 0.98); $p<0.0001$.

\section{Conclusions}

Preoperative I-FABP measurement may be used for anastomotic leakage risk assessment. Furthermore, combination of CRP and calprotectin has high diagnostic accuracy. Implementation of these markers in daily practice deserves further investigation. 


\section{Introduction}

Anastomotic leakage is a major challenge in patients undergoing colorectal surgery. Of 10,017 registered resections for colorectal cancer in the Netherlands in 2012, 691 (6.9\%) were complicated by anastomotic leakage ${ }^{1}$, and in high-risk patients incidence rates can increase to $18 \%{ }^{2}$. Anastomotic leakage is associated with high morbidity ${ }^{3}$, mortality ${ }^{46}$, reoperation ${ }^{7}$, and duration of hospitalization ${ }^{6}$. In cancer, anastomotic leakage is related to reduced disease-specific survival and higher recurrence rates ${ }^{7-10}$.

Delay in recognizing anastomotic leakage after colorectal surgery is associated with increased mortality ${ }^{11}$ 12. Early clinical presentation is heterogeneous and often nonspecific. Anastomotic leakage can present as peritonitis, localized fluid collections or subclinical leakage detected by contrast radiology. Furthermore, nonspecific symptoms including fever, absence of bowel action, and diarrhea only become apparent on postoperative day 4-7 ${ }^{11}$. Abdominal CT scan, the current standard for diagnosis of anastomotic leakage, yields low sensitivity, which may delay the diagnosis and appropriate treatment of anastomotic leakage ${ }^{13}$. Therefore, there is an urgent need for accurate diagnostic markers of anastomotic leakage at an early stage following colorectal surgery to decrease delay of diagnosis and its unfavourable sequelae.

In this prospective cohort study, plasma markers of inflammation and intestinal damage in anastomotic leakage diagnosis are investigated. C-reactive protein (CRP) has been proposed to diagnose anastomotic leakage after colorectal surgery in a variety of studies on postoperative day $2-3{ }^{14-19}$. However, results are not unambiguously persuasive showing diagnostic accuracy with about 70$80 \%$ sensitivity and specificity. Calprotectin, a heterodimeric peptide (36 kDa), constitutes about $60 \%$ of the cytosol proteins of neutrophils ${ }^{20}$. As a marker of neutrophil activation, calprotectin may be an interesting marker for exaggerated inflammation early upon anastomotic leakage. The cytokine IL-6 is found to be up regulated in peritoneal fluid during the first three postoperative days in patients developing anastomotic leakage ${ }^{21}$, although the diagnostic accuracy of IL-6 measurement in plasma is yet unknown. Intestinal fatty acid binding protein (I-FABP) ${ }^{22}$, ileal bile acid binding protein (I-BABP) ${ }^{23}$ and liver fatty acid binding protein (L-FABP) ${ }^{24}$ are proteins expressed in enterocytes and are wellknown plasma markers of enterocyte damage. The presence of I-FABP is limited to mature enterocytes of the small and large intestine. I-BABP is solely expressed 
in the ileum, whereas L-FABP is expressed in small and large intestine, liver, and kidney epithelial cells. These intestinal cell damage markers have never been investigated in the context of anastomotic leakage diagnosis.

In this prospective study, the diagnostic accuracy of plasma markers of inflammation and intestinal damage to predict anastomotic leakage after colorectal surgery are investigated.

\section{Methods}

\section{Patients}

Ninety consecutive patients scheduled to undergo colorectal surgery with primary anastomosis were prospectively enrolled at a single teaching hospital (Orbis Medical Center, Sittard, the Netherlands), between April 2011 and July 2013. Patients with inflammatory bowel disease were excluded, for this could influence plasma levels of inflammatory biomarkers. None of the enrolled patients received non-steroidal anti-inflammatory drugs (NSAIDs). NSAIDs have been omitted from routine analgesia in the enrolling hospital, since these drugs are associated with increased leak rates ${ }^{25}$. Moreover, no patients using corticosteroids were included, for this could have influenced marker levels. All patients gave written informed consent and the study was approved by the medical ethical committee Atrium-Orbis-Zuyd, Heerlen, the Netherlands. The study was conducted according to the revised version of the Declaration of Helsinki (October 2008, Seoul). Patient characteristics (sex, age, BMI, length of hospital stay, tumour location) and surgical characteristics (approach, type, anastomotic technique, ostomy, operation time, blood loss) were collected. A fast-track Enhanced Recovery After Surgery (ERAS) protocol was followed in all patients, according to which venous blood was routinely collected at postoperative day 1 and 3. Both laparoscopic and open resections were performed.

\section{Definition of anastomotic leakage}

Clinically relevant anastomotic leakage was defined as extraluminal presence of contrast fluid on contrast-enhanced CT scans and/or leakage when relaparotomy was performed, requiring reintervention. Indications for CT or relaparotomy were based upon clinical presentation, including fever, tachycardia, tachypnea, low saturation, low urinary production, abdominal pain, and signs of ileus or gastric retention. 


\section{Blood sampling}

Venous blood samples were taken at hospital admission (preoperative sample) and daily starting at the first postoperative day, until discharge. Blood samples were immediately cooled on ice, centrifuged at 3500 rotations per minute for 15 minutes and plasma samples were stored at $-80^{\circ} \mathrm{C}$ until batch analysis. All analyses were performed by one person (A.v.B.) after inclusion of all patients; therefore the treating clinicians were not aware of the values. The technician was not aware of patient characteristics.

\section{CRP, calprotectin and IL-6 measurement}

CRP plasma levels were determined by an enzyme-linked immunosorbent assay (ELISA) developed at our institute, as described before (lower detection limit: $100 \mathrm{pg} / \mathrm{mL})^{26}$. Calprotectin plasma concentration was measured using the commercially available calprotectin ELISA (lower detection limit $46.8 \mathrm{ng} / \mathrm{ml}$, Hycult Biotechnology Uden, the Netherlands). IL-6 levels were measured by ELISA as previously described (lower detection limit: $10 \mathrm{pg} / \mathrm{mL}$ ) ${ }^{27}$.

\section{FABP measurement}

I-FABP plasma levels were determined using an in-house ELISA that selectively detects human I-FABP (lower detection limit: $25 \mathrm{pg} / \mathrm{mL}$ ). Plasma I-BABP was measured as previously described (lower detection limit: $0.1 \mathrm{ng} / \mathrm{mL}$ ) ${ }^{28}$. L-FABP plasma levels were determined using a commercially available enzyme-linked immunosorbent assay (Hycult Biotechnology, Uden, the Netherlands, lower detection limit: $2 \mathrm{ng} / \mathrm{mL}$ ).

\section{Statistical analysis}

Normality was tested by Kolmogorov-Smirnov. Student's t-test was used for between group comparisons for continuous data. Dichotomous variables were compared using Pearson's chi-squared test. All data are presented as mean and standard error of the mean (SEM). Receiver operating characteristic (ROC) curves were used to calculate the accuracy of the studied markers predicting anastomotic leakage. The ideal cut-off value for diagnosing anastomotic leakage was defined as the cut-off value with maximum sum of sensitivity and specificity. 
Overall diagnostic accuracy was represented by the area under the curve (AUC). As many factors influence anastomotic leakage rates ${ }^{29}$, possible confounding factors were analysed by logistic regression analysis.

To determine the accuracy of combined markers detecting anastomotic leakage, logistic regression analysis was performed and predicted probabilities were then plotted in ROC curves. The best cut-off point of predicted probabilities $(P)$ was defined as the cut-off point with maximum sum of sensitivity and specificity. To calculate the linear function describing all combinations of ideal cut-off values for combined markers in the detection of anastomotic leakage, the cut-off point $(P)$ was used in the following equation: $\operatorname{Ln}(P /(1-P))=B_{0}+B_{1} X_{1}+B_{2} X_{2}$; in which $B_{0}$ represents the constant of the logistic regression analysis and $B_{1}$ and $B_{2}$ represent the logistic regression coefficients of the markers. By calculating coordinates of intersections with the $x$ - and $y$-axis, the linear function describing the cut-off line to discriminate anastomotic leakage could be determined ${ }^{30}$.

Sample size was calculated based on CRP levels at POD4 ${ }^{31}$. Mean CRP levels in the anastomotic leakage group were estimated at 200 (SD, 100) mg/L, and $75(\mathrm{SD}, 100) \mathrm{mg} / \mathrm{L}$ in the group without anastomotic leakage. With $\alpha=0.05$ and $1-\beta=0.80$, a total sample size of 80 was calculated, including 6 cases of anastomotic leakage at anastomotic leakage incidence of $8 \%$.

Statistical analyses were performed with Prism 5.0 for Windows (GraphPad Software Inc. San Diego, CA) and SPSS 20.0 for Windows (SPSS Inc. Chicago, IL). STARD statement for reporting studies of diagnostic accuracy was used in this study ${ }^{32}$.

TABLE 1. Patient characteristics

\begin{tabular}{llll}
\hline & & Number of patients & Mean (SEM) \\
\hline Sex & Male & $54(64.3 \%)$ & \\
Age (years) & Female & $30(35.7 \%)$ & $65(1)$ \\
BMl $\left(\mathrm{kg} / \mathrm{m}^{2}\right)$ & $>70$ & $24(28.6 \%)$ & $26.6(0.5)$ \\
& $>30$ & $13(15.5 \%)$ & $8.0(0.7)$ \\
$\begin{array}{l}\text { Length of hospital } \\
\text { stay (days) }\end{array}$ & & & \\
$\begin{array}{l}\text { Tumor location } \\
\text { Colon }\end{array}$ & Rectum/Sigmoid & $40(57.6 \%)$ & \\
& & &
\end{tabular}




\section{Results}

\section{Patients}

Of ninety enrolled patients, three were excluded as it was decided not to perform a primary anastomosis after patient inclusion, and three were excluded for retracting informed consent. The remaining 84 patients had a mean age of 65 (SEM, 1.4) years, and 54 (64\%) were males. Baseline and operative characteristics are listed in Table 1 and Table 2, respectively.

Eight patients (10\%) developed anastomotic leakage which became apparent at median postoperative day (POD) 6 (range, 3-10 days). Six patients (75\%) with anastomotic leakage required surgical reintervention, the remaining two patients were treated with intravenous antibiotics. No radiological drainage was performed in any case of anastomotic leakage. Three patients (4\%) required surgical reintervention for fascial dehiscence, and in three patients (4\%), anastomotic leakage was clinically suspected but not found at relaparotomy. Mortality was $2 \%$ (1 patient with anastomotic leakage).

TABLE 2. Surgery characteristics

\begin{tabular}{llll}
\hline & & Number of patients & Mean (SEM) \\
\hline Surgical approach & Open & $48(57.1 \%)$ & \\
& Laparoscopy & $36(42.9 \%)$ & \\
Type of surgery & Right colectomy & $25(29.8 \%)$ & \\
& Left colectomy & $37(44 \%)$ & \\
& Rectal resection & $22(26.2 \%)$ & \\
Anastomotic & & & \\
technique & Handsewn & $35(41.7 \%)$ & $134(8)$ \\
& Mechanical & $49(58.3 \%)$ & $226(27)$ \\
$\begin{array}{l}\text { Ostomy } \\
\text { Operative time }\end{array}$ & & & \\
(minutes) & & & \\
Blood loss $(\mathrm{mL})$ & & & \\
\hline
\end{tabular}

Logistic regression of possible confounding factors

Factors influencing anastomotic leakage rates were considered as possible confounders en were therefore entered in a univariate logistic regression analysis. None of the analysed factors were significantly associated with anastomotic 
leakage in this cohort (age: odds ratio (OR) 1.00 (95\% confidence interval (Cl) 0.94 - 1.06), $p=0.94$; sex: OR 1.76 (95\% Cl 0.33 - 9.35), $p=0.51$; ASA: OR 0.60 (95\% $\mathrm{Cl} 0.10-3.78), p=0.59$; location of anastomosis: OR 0.29 (95\% Cl $0.06-1.54)$, $p=0.15$; and diverting ostomy: OR 0 ( $95 \% \mathrm{Cl} 0$ - infinite), $p=1.00)$. Therefore, none of these factors were considered as confounders.

\section{CRP, calprotectin and IL-6 measurement}

Mean CRP levels in patients with anastomotic leakage were elevated at POD 4 compared to CRP levels in patients without anastomotic leakage: 415 (221) mg/L compared to 119 (19) mg/L, $p=0.002$ (Figure 1). Overall diagnostic accuracy of

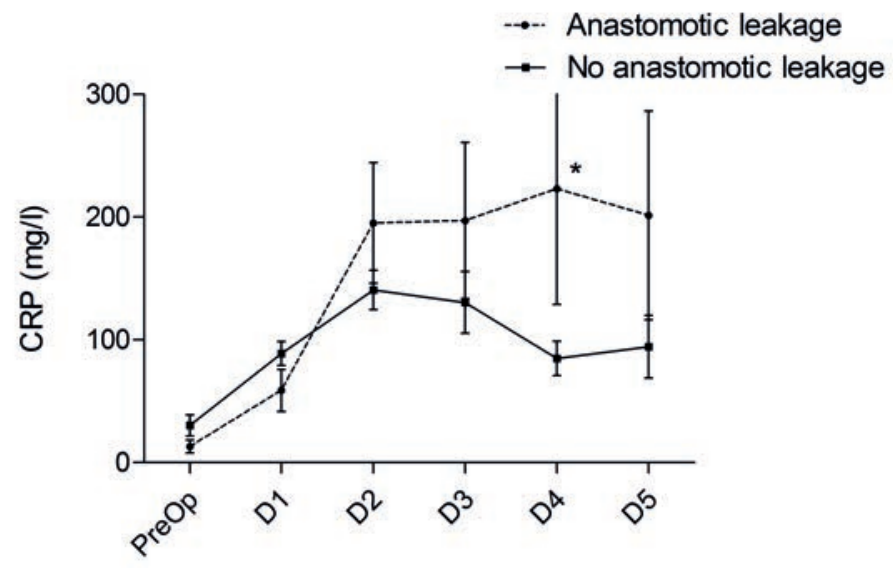

FIGURE 1. Plasma CRP levels (mg/L). Values are mean \pm SEM.

CRP to detect anastomotic leakage at POD 4 in these patients using ROC curve analysis was described by area under the curve (AUC) of 0.82 (95\% confidence interval (Cl), 0.69 - 0.96). The optimal cut-off value of $99 \mathrm{mg} / \mathrm{L}$ produced sensitivity, 100\%; specificity, 64\%; positive likelihood ratio (LR+), $2.78(95 \% \mathrm{Cl}$, 1.87 - 3.65); negative likelihood ratio (LR-), 0 (95\% Cl, 0 - 1.24).

Mean calprotectin levels were elevated in patients with anastomotic leakage at POD $2(588(62) \mathrm{ng} / \mathrm{mL})$ compared with patients without anastomotic leakage (366 (25) ng/mL, p=0.006). AUC of the ROC curve was $0.82(95 \% \mathrm{Cl}, 0.68-0.95)$ (Figure 2). A cut-off value of $411 \mathrm{ng} / \mathrm{mL}$ yielded sensitivity, 100\%; specificity, 58\%; LR+, 2.38 (95\% Cl, 1.60 - 3.09); LR-, 0 (95\% Cl, 0 - 1.61). At POD 3, diagnostic accuracy increased to AUC 0.92 (95\% Cl, 0.83 - 1.00). A cut-off value of $544 \mathrm{ng} /$ mL produced sensitivity, 86\%; specificity, 88\%; LR+, 7.17 (95\% Cl, 3.55 - 14); LR-, 


\section{$\rightarrow$ Anastomotic leakage}

$\rightarrow$ No anastomotic leakage

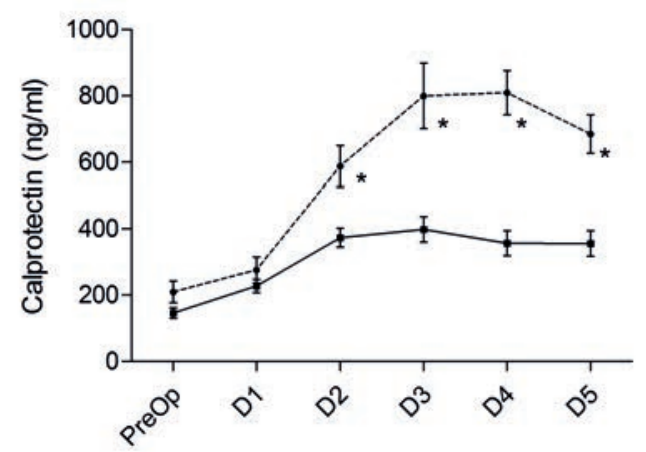

FIGURE 2. Plasma calprotectin levels $(\mathrm{ng} / \mathrm{mL})$. Values are mean \pm SEM.

0.16 (95\% Cl, 0.03 - 1.01). At POD 4, diagnostic accuracy further increased to AUC 0.96 (95\% Cl, 0.90 - 1.00). With a cut-off value of $541 \mathrm{ng} / \mathrm{mL}$, this resulted in sensitivity, 100\%; specificity, 91\%; LR+, 11 (95\% Cl, 4.68 - 20); LR-, 0 (95\% Cl, 0 - 1.02).

IL-6 levels were not different between patients with and without anastomotic leakage at any time point (data not shown).

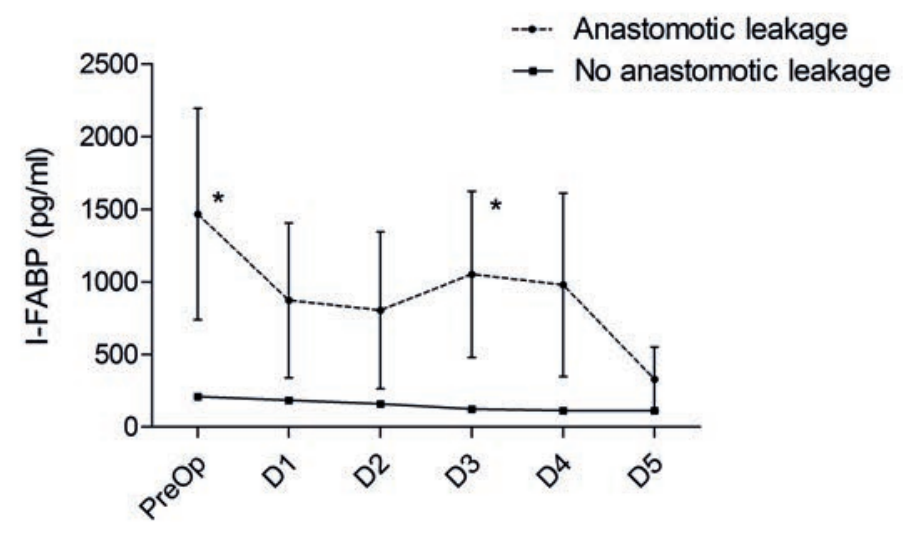

FIGURE 3. Plasma I-FABP levels $(\mathrm{pg} / \mathrm{mL})$. Values are mean \pm SEM. 


\section{Combination of CRP and calprotectin at postoperative day 3 and 4}

When CRP levels at POD 3 were combined with calprotectin measurement on POD 3 to predict anastomotic leakage, a cut-off line was calculated with sensitivity, 100\%; specificity, 89\%; LR+, 9.09 (95\% Cl, 4.34 - 16); and LR-, 0 (95\% Cl, 0 $0.89) ; p<0.0001$. The cut-off line was described by the linear function: [Calprotectin $(\mathrm{ng} / \mathrm{mL})]+0.83 \cdot[\mathrm{CRP}(\mathrm{mg} / \mathrm{L})]=707$;

which means that a positive test is obtained when any combination of CRP and calprotectin levels at POD 3 in this formula results in a value $>707$. AUC of the ROC curve was $0.93(95 \% \mathrm{Cl} 0.87-1.00)$.

By measuring CRP and calprotectin at POD 3, diagnosis of anastomotic leakage could therefore be shortened by median 3 (range, $0-7$ ) days.

At POD 4, this cut-off line yielded comparable accuracy compared to POD 3 (sensitivity, 100\%; specificity, 90\%; LR+, 10 (95\% Cl, 4.6 - 18); and LR-, 0 (95\% $\mathrm{Cl}, 0-0.88) ; p=0.001$ ), with AUC of the ROC curve 0.97 (95\% Cl $0.92-1.00)$.

\section{FABP measurement}

Preoperative I-FABP levels were elevated in patients who developed anastomotic leakage compared to controls (1466 (728) pg/mLand $190(17) \mathrm{pg} / \mathrm{mL}$, respectively, $p<0.0001)$ and remained elevated over time, however only significantly at POD 3 (Figure 3). A preoperative cut-off value of $882 \mathrm{pg} / \mathrm{mL}$ yielded sensitivity, 50\%; specificity, 100\%; LR+, infinite (95\% Cl, 4.01 - infinite); LR-, 0.50 (95\% Cl, 0.26 - 0.98).

Plasma I-BABP and L-FABP levels were not different between groups at any time point (data not shown).

\section{Discussion}

A plasma marker for early detection of anastomotic leakage after colorectal surgery is lacking. In this study, the plasma neutrophil activation marker calprotectin showed high sensitivity and specificity from POD2 and onwards, especially when combined with the conventional acute phase protein CRP. CRP has been described widely as a useful diagnostic test for anastomotic leakage with sensitivity rates varying from $73-91 \%$ and specificity $60-86 \%$ on postoperative day $3-5$, with cut-off values ranging from $100-147 \mathrm{mg} / \mathrm{L}{ }^{15,17,33,34}$. A recent meta-analysis of six studies including 1,832 patients indicated that CRP levels at postoperative day 4 had a pooled sensitivity of $68 \%$, specificity of $83 \%$ and a negative predictive value of 
$89 \%$ for predicting infectious complications ${ }^{35}$. In concordance, with sensitivity $100 \%$, the negative predictive value in our study was $100 \%$ as well. Measuring CRP postoperatively to monitor the development of inflammatory complications is considered standard care in many hospitals. However, a recent study demonstrated a low predictive value of CRP for the development of anastomotic leakage after laparoscopic colorectal surgery ${ }^{36}$. Moreover, a high negative predictive value is useful to rule out the development of complications and to confirm safety of discharge from the hospital, yet the decision for relaparotomy is based on the positive predictive value or positive likelihood ratio, which is low for CRP. The abovementioned indicates, although not indisputable, that routine CRP measurement can guide safe hospital discharge. To confirm the decision for reintervention, other tests are needed.

Calprotectin has not been investigated before as a predictor for anastomotic leakage which was clinically detectable at POD 3-10. In this study, calprotectin was superior over CRP in the detection of anastomotic leakage, by both earlier (POD 2-3 vs. POD 4) and more accurate (AUC 0.92 at POD 3 vs. 0.82 at POD 4) detection. Furthermore, the best diagnostic accuracy was obtained when CRP and calprotectin measurement at POD 3 were combined, which still is earlier $(0-7$, median 3 days) than clinical signs. As specificity increased when adding calprotectin measurement, false-positive test results decreased dramatically.

IL-6 plasma levels were not different between patients with and without anastomotic leakage. Several studies have found a significant difference when measuring cytokines in peritoneal fluid ${ }^{21}$. Apparently, these differences are not reflected by circulating cytokine levels.

It can only be speculated why calprotectin is a better marker for anastomotic leakage than CRP and IL-6. As part of the innate immune response, neutrophils provide a first line of defense against bacterial infection, and are recruited to sites of infection or inflammatory stimuli within minutes, with a peak by $24-48$ hours ${ }^{37}$, ${ }^{38}$. Being a neutrophil activation product, calprotectin may therefore be increased early after anastomotic leakage, while CRP reflects the systemic inflammatory response, which becomes apparent later on. Neutrophil count has earlier been described to have no additive effect on CRP to predict anastomotic leakage after laparoscopic sleeve gastrectomy ${ }^{39}$. However, the complete neutrophil count was used in this study, while calprotectin reflects the amount of activated neutrophils. 
Of different FABP-types, only I-FABP levels showed significant differences between patients with and without anastomotic leakage. This is remarkable, as L-FABP is the predominant FABP type in colonic tissue, where I-FABP is only present in small amounts ${ }^{24}$. It is also noteworthy that nonspecific markers (CRP, calprotectin) did detect anastomotic leakage postoperatively, while specific intestinal markers (FABPs) did not. It could be assumed that intestinal epithelial damage is relatively limited in anastomotic leakage compared to other intestinal pathology such as ischemia-reperfusion injury ${ }^{40}$ and necrotizing enterocolitis ${ }^{41}$, and that excessive inflammation is the predominant pathological process in anastomotic leakage.

Moreover, I-FABP was already elevated preoperatively in patients who ultimately developed anastomotic leakage. These findings may point at generalized intestinal impairment, predisposing patients to the development of anastomotic leakage. The positive likelihood ratio of preoperative I-FABP levels at a cut-off value of 882 $\mathrm{pg} / \mathrm{mL}$ indicates a post-test probability of $100 \%$ when a positive test is obtained. I-FABP levels may therefore be used for preoperative risk assessment. Elevated I-FABP levels predict a $100 \%$ chance for developing anastomotic leakage and in these patients treatment options could be adapted, for example by the construction of a diverting ileo- or colostomy or performing a Hartmann's procedure.

In the current study, all patients undergoing colorectal resection including those with a diverting ostomy were analysed. Although excluding patients with an ostomy would generate a more clean cohort, the current results are applicable to the complete population of patients undergoing colorectal surgery with primary anastomosis.

A limitation of all studies investigating anastomotic leakage including the present study is the lack of a true gold standard. By some authors, the gold standard is considered evident leakage of luminal contents or presence of a perianastomotic abscess on laparotomy ${ }^{13}$. However some cases of anastomotic leakage develop subclinically or do not require surgical intervention. Intraluminal contrast-enhanced CT scanning is preferred as the main imaging method to diagnose anastomotic leakage in many hospitals, although sensitivity and specificity of CT scans vary greatly ${ }^{13}$. In this study, some subclinical leakages may have been missed, since CT scan was not performed in every patient. However, the primary diagnostic aim is to detect anastomotic leaks which are clinically relevant and therefore require treatment, which are all included. Furthermore, with regards to radiation exposure, it is not ethical to perform a CT scan every day solely for study purposes. Another limitation for interpretation of the results is the observational design of 
this study, which presents a phase I diagnostic study, according to Sackett and Haynes ${ }^{42}$. The next appropriate step would be to conduct a phase II diagnostic study, which answers the question: "Are patients with certain test results more likely to have the target disorder", which changes the direction of interpretation. This question can only truly be answered in a new prospective dataset, using the cut-off values defined in the current study to predict anastomotic leakage. The following step would be to guide treatment based on the preoperative I-FABP, and postoperative CRP and calprotectin, preferentially in a large multicenter study. High preoperative I-FABP values can filter out half of patients who develop anastomotic leakage without false-positive results (sensitivity 50\%, with post-test probability $100 \%)$, and for whom a different surgical approach can be considered. The best diagnostic accuracy is obtained when CRP and calprotectin are combined at POD 3. CRP/calprotectin measurement on POD 3 can therefore aid to determine which patients can be discharged safely and which patients should be monitored intensively. In the latter case, relaparotomy should be considered as well. Future interventional studies should investigate whether treatment strategies based on these markers leads to prevention and better management of anastomotic leakage after colorectal surgery. 


\section{References}

1. Jaarrapportage 2012: uitkomst van zorg registratie. Dutch Surgical Colorectal Audit, 2012.

2. Tan WP, Talbott VA, Leong QQ, et al. American Society of Anesthesiologists class and Charlson's comorbidity index as predictors of postoperative colorectal anastomotic leak: a single-institution experience. J Surg Res 2013;184:115-9.

3. Makela JT, Kiviniemi H, Laitinen S. Risk factors for anastomotic leakage after left-sided colorectal resection with rectal anastomosis. Dis Colon Rectum 2003;46:653-60.

4. Karanjia ND, Corder AP, Bearn P, et al. Leakage from stapled low anastomosis after total mesorectal excision for carcinoma of the rectum. Br J Surg 1994;81:1224-6.

5. den Dulk M, Noter SL, Hendriks ER, et al. Improved diagnosis and treatment of anastomotic leakage after colorectal surgery. Eur J Surg Oncol 2009;35:420-6.

6. Golub R, Golub RW, Cantu R, Jr., et al. A multivariate analysis of factors contributing to leakage of intestinal anastomoses. J Am Coll Surg 1997;184:364-72.

7. Walker KG, Bell SW, Rickard MJ, et al. Anastomotic leakage is predictive of diminished survival after potentially curative resection for colorectal cancer. Ann Surg 2004;240:255-9.

8. McArdle CS, McMillan DC, Hole DJ. Impact of anastomotic leakage on long-term survival of patients undergoing curative resection for colorectal cancer. $\mathrm{Br} \mathrm{J}$ Surg 2005;92:1150-4.

9. Bell SW, Walker KG, Rickard MJ, et al. Anastomotic leakage after curative anterior resection results in a higher prevalence of local recurrence. Br J Surg 2003;90:12616.

10. McMillan DC, McArdle CS, Morrison DS. A clinical risk score to predict 3-, 5- and 10year survival in patients undergoing surgery for Dukes B colorectal cancer. Br J Cancer 2010;103:970-4.

11. Alves A, Panis $Y$, Trancart D, et al. Factors associated with clinically significant anastomotic leakage after large bowel resection: multivariate analysis of 707 patients. World J Surg 2002;26:499-502.

12. Macarthur DC, Nixon SJ, Aitken RJ. Avoidable deaths still occur after large bowel surgery. Scottish Audit of Surgical Mortality, Royal College of Surgeons of Edinburgh. Br J Surg 1998;85:80-3.

13. Kornmann VN, Treskes N, Hoonhout LH, et al. Systematic review on the value of CT scanning in the diagnosis of anastomotic leakage after colorectal surgery. Int J Colorectal Dis 2013;28:437-45.

14. Singh PP, Zeng IS, Srinivasa S, et al. Systematic review and meta-analysis of use of serum C-reactive protein levels to predict anastomotic leak after colorectal surgery. $\mathrm{Br}$ J Surg 2013.

15. Almeida AB, Faria G, Moreira $\mathrm{H}$, et al. Elevated serum C-reactive protein as a predictive factor for anastomotic leakage in colorectal surgery. Int J Surg 2012;10:87-91. 
16. Lane JC, Wright S, Burch J, et al. Early prediction of adverse events in enhanced recovery based upon the host systemic inflammatory response. Colorectal Dis 2013;15:22430.

17. Lagoutte N, Facy O, Ravoire A, et al. C-reactive protein and procalcitonin for the early detection of anastomotic leakage after elective colorectal surgery: Pilot study in 100 patients. J Visc Surg 2012;149:e345-9.

18. Platt JJ, Ramanathan ML, Crosbie RA, et al. C-reactive Protein as a Predictor of Postoperative Infective Complications after Curative Resection in Patients with Colorectal Cancer. Ann Surg Oncol 2012;19:4168-77.

19. Korner H, Nielsen HJ, Soreide JA, et al. Diagnostic accuracy of C-reactive protein for intraabdominal infections after colorectal resections. J Gastrointest Surg 2009;13:1599-606.

20. Fagerhol MK. Calprotectin, a faecal marker of organic gastrointestinal abnormality. Lancet 2000;356:1783-4.

21. Cini C, Wolthuis A, D'Hoore A. Peritoneal fluid cytokines and matrix metalloproteinases as early markers of anastomotic leakage in colorectal anastomosis: a literature review and meta-analysis. Colorectal Dis 2013;15:1070-7.

22. Veerkamp JH, Paulussen RJ, Peeters RA, et al. Detection, tissue distribution and (sub) cellular localization of fatty acid-binding protein types. Mol Cell Biochem 1990;98:118.

23. Watanabe K, Hoshi N, Tsuura Y, et al. Immunohistochemical distribution of intestinal 15 kDa protein in human tissues. Arch Histol Cytol 1995;58:303-6.

24. Pelsers MM, Namiot Z, Kisielewski W, et al. Intestinal-type and liver-type fatty acidbinding protein in the intestine. Tissue distribution and clinical utility. Clin Biochem 2003;36:529-35.

25. Gorissen KJ, Benning D, Berghmans T, et al. Risk of anastomotic leakage with nonsteroidal anti-inflammatory drugs in colorectal surgery. Br J Surg 2012;99:721-7.

26. van Dielen FM, van't Veer C, Schols AM, et al. Increased leptin concentrations correlate with increased concentrations of inflammatory markers in morbidly obese individuals. Int J Obes Relat Metab Disord 2001;25:1759-66.

27. Hanssen SJ, Derikx JP, Vermeulen Windsant IC, et al. Visceral injury and systemic inflammation in patients undergoing extracorporeal circulation during aortic surgery. Ann Surg 2008;248:117-25.

28. Derikx JP, Blijlevens NM, Donnelly JP, et al. Loss of enterocyte mass is accompanied by diminished turnover of enterocytes after myeloablative therapy in haematopoietic stem-cell transplant recipients. Ann Oncol 2009;20:337-42.

29. Bakker IS, Grossmann I, Henneman D, et al. Risk factors for anastomotic leakage and leak-related mortality after colonic cancer surgery in a nationwide audit. Br J Surg 2014;101:424-32; discussion 432.

30. Reisinger KW, Van der Zee DC, Brouwers HA, et al. Noninvasive measurement of fecal calprotectin and serum amyloid A combined with intestinal fatty acid-binding protein in necrotizing enterocolitis. J Pediatr Surg 2012;47:1640-5. 
31. MacKay GJ, Molloy RG, O'Dwyer PJ. C-reactive protein as a predictor of postoperative infective complications following elective colorectal resection. Colorectal Dis 2011;13:583-7.

32. Bossuyt PM, Reitsma JB, Bruns DE, et al. The STARD statement for reporting studies of diagnostic accuracy: explanation and elaboration. Clin Chem 2003;49:7-18.

33. Garcia-Granero A, Frasson M, Flor-Lorente B, et al. Procalcitonin and C-reactive protein as early predictors of anastomotic leak in colorectal surgery: a prospective observational study. Dis Colon Rectum 2013;56:475-83.

34. Scepanovic MS, Kovacevic B, Cijan V, et al. C-reactive protein as an early predictor for anastomotic leakage in elective abdominal surgery. Tech Coloproctol 2013;17:541-7.

35. Warschkow R, Beutner U, Steffen T, et al. Safe and early discharge after colorectal surgery due to C-reactive protein: a diagnostic meta-analysis of 1832 patients. Ann Surg 2012;256:245-50.

36. Pedersen T, Roikjaer O, Jess P. Increased levels of C-reactive protein and leukocyte count are poor predictors of anastomotic leakage following laparoscopic colorectal resection. Dan Med J 2012;59:A4552.

37. Szabady RL, McCormick BA. Control of neutrophil inflammation at mucosal surfaces by secreted epithelial products. Front Immunol 2013;4:220.

38. Fournier BM, Parkos CA. The role of neutrophils during intestinal inflammation. Mucosal Immunol 2012;5:354-66.

39. Albanopoulos K, Alevizos L, Natoudi M, et al. C-reactive protein, white blood cells, and neutrophils as early predictors of postoperative complications in patients undergoing laparoscopic sleeve gastrectomy. Surg Endosc 2013;27:864-71.

40. Schellekens DH, Grootjans J, Dello SA, et al. Plasma Intestinal Fatty Acid-Binding Protein Levels Correlate With Morphologic Epithelial Intestinal Damage in a Human Translational Ischemia-reperfusion Model. J Clin Gastroenterol 2013.

41. Derikx JP, Evennett NJ, Degraeuwe PL, et al. Urine based detection of intestinal mucosal cell damage in neonates with suspected necrotising enterocolitis. Gut 2007;56:14735.

42. Sackett DL, Haynes RB. The architecture of diagnostic research. BMJ 2002;324:53941. 




\section{Part 3 \\ Pathophysiologic aspects of necrotizing enterocolitis (NEC)}

The third aim was to characterize gut-specific biomarkers in the context of gut maturation to gain better understanding of the pathophysiology of NEC. NEC is the most frequent emergency indication for pediatric gastrointestinal surgery, which carries high mortality. The fulminant character of NEC is one of the explanations for its unfavorable outcome. Moreover, NEC is a disease typically present in premature neonates, who represent a highly vulnerable population, such as frail elderly who were described in part 1. Premature neonates are therefore not quite suitable to undergo surgery, however in case of NEC surgical intervention may be inevitable. Better understanding of gut immaturity in the context of NEC is mandatory to develop strategies to prevent NEC and to improve outcomes of NEC patients. In chapter 8, a marker of intestinal cell damage was further explored in both humans and sheep, answering the question whether it can used to assess gut maturity. In chapter 9 , a study of human neonates is described in which it was investigated whether human milk increases gut maturation compared to formula milk. 

Chapter 8. Intestinal fatty acid binding protein (I-FABP): a possible marker for gut maturation

Kostan W. Reisinger, Marieke Elst, Joep P.M. Derikx, Peter G.J. Nikkels, Bart de Vries, Marlou P.M. Adriaanse, Reint K. Jellema, Boris W.W. Kramer, Tim G.A.M. Wolfs. Pediatric Research 2014. 76(3):261-8 


\begin{abstract}
Background

Gut immaturity is linked with postnatal intestinal disorders. However, biomarkers to assess the intestinal developmental stage around birth are lacking. The aim of this study was to gain more insight on intestinal-fatty acid binding protein (I-FABP) as indicator of gut maturity.
\end{abstract}

\title{
Methods
}

Antenatal I-FABP distribution and release was investigated in extremely premature, moderately premature and term lambs and these findings were verified in urinary human samples. Ileal I-FABP distribution was confirmed in autopsy material within $24 \mathrm{~h}$ postnatally.

\section{Results}

Median [range] serum I-FABP levels were lower in extremely premature lambs compared to moderately premature lambs (156 [50.0-427] vs. 385 [100-1,387] $\mathrm{pg} / \mathrm{ml}, \mathrm{p}=0.02)$. Contrarily, median early postnatal urine I-FABP levels in human infants were higher in extremely premature compared to moderately premature and term neonates $(1,219$ [203-15,044] vs. 256 [50-1,453] and vs. 328 [961,749) $\mathrm{pg} / \mathrm{ml}, \mathrm{p}=0.008$ and $\mathrm{p}=0.04$, respectively). I-FABP expression was most prominent in non-vacuolated enterocytes and increased with rising gestational age in ovine and human tissue samples. The epithelial distribution pattern changed from a phenotype displaying I-FABP positive enterocytes merely in the crypts early in gestation into a phenotype with I-FABP expressing cells exclusively present in the villus tips at term in ovine and human tissue.

\section{Conclusion}

In this ovine and human study, increasing gestational age is accompanied by an increase in I-FABP tissue content. Cord I-FABP levels correlate with gestation in ovine fetuses identifying I-FABP as a marker for gut maturation. Raised postnatal urine I-FABP levels in preterm human infants may indicate intestinal injury and/or inflammation in utero. 


\section{Introduction}

Immaturity of the gut plays a pivotal role in the development of necrotizing enterocolitis (NEC), gut-derived sepsis and feeding problems ${ }^{1}$. The underlying mechanisms remain poorly understood, but increased intestinal permeability and inadequate intestinal immune responses in preterm neonates are associated with gastrointestinal disorders ${ }^{2-4}$. In order to define the maturational stage of the gut, markers of gut maturity are required.

A good candidate may be intestinal fatty acid binding protein (I-FABP), which is a sensitive and specific enterocyte marker ${ }^{5-7}$. It is released into the circulation as soon as the enterocyte's cell membrane integrity is compromised and it can be detected in urine ${ }^{5,8}$, making it a suitable marker for intestinal pathologies such as NEC and coeliac disease ${ }^{9,10}$. Moreover, I-FABP reflects enterocyte turnover and is exclusively present in mature enterocytes of the small and large intestine 11, 12. During early fetal life, the intestinal epithelial lining consists of vacuolated, immature enterocytes, as shown in animal models and in the human gut ${ }^{13-15}$. These immature cells gradually disappear with increasing gestational age and are completely replaced by mature enterocytes shortly after birth ${ }^{14,16}$. More insight on I-FABP distribution and its release into the circulation as an indicator of gut maturity can aid in the management of gut-associated problems in premature neonates. We hypothesized that I-FABP distribution and release is gestation dependent. To test this hypothesis, I-FABP distribution and release were evaluated during gestation in utero in fetal lambs, since the developmental biology of sheep is closest to the human development for most major organs including the gut ${ }^{17}$. In addition, the postnatal distribution and release of I-FABP were tested in preterm neonates.

\section{Materials and methods}

\section{Animals}

This study was approved by the Animal Ethics Committee of the University of Maastricht, the Netherlands. Time-mated pregnant ewes and fetuses were humanely euthanized by intravenous pentobarbital (150-200 mg/kg) injection and lambs were delivered by caesarian section either extremely preterm (120-125 days GA, comparable to $<28$ weeks GA in humans), preterm (130-135 days GA, comparable to 28-37 weeks GA in humans) or term (140-145 days GA, comparable to $37-42$ weeks GA in humans). Samples of the small intestine were collected and 
both snap frozen and fixated in formalin. Cord blood was immediately sampled from the umbilical artery, cooled on ice, centrifuged at 3,500 rotations per minute for 15 minutes and serum samples were stored at $-80^{\circ} \mathrm{C}$ until batch analysis.

\section{Patients}

Neonates admitted to the neonatal intensive care unit at the Maastricht University Medical Centre between July 2007 and July 2008 were eligible for inclusion, if not admitted after the third postnatal day. Neonates with asphyxia or those developing gastrointestinal events such as NEC or feeding problems were excluded retrospectively at NICU discharge. Infants were divided into an extremely premature group (<28 weeks of gestation), a moderately premature group (28-37 weeks), and a term group (37-42 weeks). Eligibility and inclusion numbers are outlined in Table 4. It was anticipated that only 25 extremely premature infants would be admitted to the NICU during the study period, therefore the 25 first consecutive infants of all three groups were approached for consent. None of the approached parents refused consent, however some infants were excluded retrospectively based on the exclusion criteria mentioned above. Written informed consent was obtained from both parents. The study was conducted with approval from the local medical ethical committee, and the principles of good clinical practice (GCP) were followed during this study.

Urine samples were collected by placement of a dental cotton roll (Henry Schein, Almere, The Netherlands) in the diaper of the neonate starting at the first day after birth. Once the roll was filled with urine, it was placed in a sterile 5 $\mathrm{mL}$ syringe (Becton Dickinson, Oxford, United Kingdom), the urine was pressed into Micronic tubes (Micronic B.V., Lelystad, the Netherlands) and stored at -20 ${ }^{\circ} \mathrm{C}$ until analysis. Two samples were collected from each patient; one on day 0-3 after birth and one on day 4-7. Enteral feeding was initiated before the first urine sample was taken.

\section{Human tissue collection}

In the pathology department database of the Maastricht and Utrecht University Medical Centres neonates who died within 24 hours after birth between January 2005 and December 2012 were identified. Of those patients in which intestinal tissue had been collected at postmortem examination, paraffin-embedded 
sections of ileal tissue were used for immunohistochemistry. Comparisons were made between extremely premature ( $<28$ weeks of gestation), moderately premature (28-37 weeks), and term (37-42 weeks) neonates.

\section{Antibodies}

The following antibodies were used for immunohistochemistry: rabbit anti-human cleaved caspase-3 (Asp175), (Cell Signaling, Danvers, MA), rabbit anti-human I-FABP (in-house developed), and mouse anti-human Ki-67 (Clone MIB-1, Dako, Glostrup, Denmark). Goat anti-rabbit IgG labelled with horseradish peroxidase (HRP) (Jackson ImmunoResearch, West Grove, PA) and biotin-conjugated swine anti-rabbit IgG (Dako) and goat anti-mouse IgG (Dako) were used as secondary antibodies.

For western blot, the following antibodies were used: rabbit anti-human I-FABP (in-house developed) and mouse anti-human $\beta$-actin clone ac15 (Sigma, St Louis, $\mathrm{MO})$. The secondary antibodies used for western blot were IRDye $800 \mathrm{cw}$ goat antirabbit (LI-COR Biosciences, Lincoln, NE) and IRDye $800 \mathrm{cw}$ goat anti-mouse (LICOR Biosciences).

\section{Immunohistochemistry}

Ovine intestinal samples were immediately immersed in $4 \%$ formaldehyde fixative (Unifix, Klinipath, Duiven, the Netherlands) and incubated for 24 hours. Tissue samples were embedded in paraffin and $4 \mu \mathrm{m}$ sections were cut. Sections were first deparaffinized in xylene and then rehydrated in graded ethanol to distilled water. Endogenous peroxidase activity was blocked with $0.5 \% \mathrm{H}_{2} \mathrm{O}_{2}$. Antigen retrieval was performed in citrate buffer at $90^{\circ} \mathrm{C}$ for 30 minutes (Ki-67, I-FABP). Unspecific binding sites were blocked using normal goat serum (Ki-67) for 30 minutes at room temperature. Slides were incubated with the primary antibody for 60 minutes at room temperature (I-FABP, caspase-3) or overnight (Ki-67) at $4{ }^{\circ} \mathrm{C}$, and then incubated with the appropriate secondary antibody for 1 hour at room temperature. The tissue sections were prepared with $\mathrm{ABC}$-complex (Ki-67, caspase-3). For I-FABP positive staining was visualized with 3-amino-9ethylcarbazole. Counterstaining of the nuclei was performed using haematoxylin. For Ki-67 and caspase-3, the staining was visualized with Nickel-sulfate 3,3-diaminobenzidine, counterstaining with Nuclear Fast Red. 


\section{ELISA}

Urinary and serum I-FABP was measured using an in-house enzyme-linked immunosorbent assay (ELISA) that selectively detects human and ovine I-FABP (standard 10-800 pg/ml, lower detection level 50 pg/ml). I-FABP concentrations are given in $\mathrm{pg} / \mathrm{ml}$. Human urine samples were batch-analyzed after inclusion of the complete cohort.

\section{Western blot}

Western blot was used for the analysis of I-FABP protein content of ovine ileal samples. First, snap frozen tissues were homogenized in RIPA buffer containing protease inhibitors ( $1 \mathrm{mM}$ PMSF, $0.1 \mathrm{U} / \mathrm{mL}$ aprotinin and $1 \mu \mathrm{g} / \mathrm{mL}$ leupeptin). Determination of the protein concentration in the final supernatants was performed using the bicinchoninic acid (BCA) protein assay. Equal amounts of total protein $\left(20 \mu \mathrm{g}\right.$ or $40 \mu \mathrm{g}$ where applicable) were heated at $100{ }^{\circ} \mathrm{C}$ for 10 minutes in SDS buffer containing $\beta$-mercaptoethanol. Proteins were separated by running the samples through a $15 \%$ SDS-polyacrylamide gel and transferred to a nitrocellulose membrane. After a blocking step the membranes were incubated with the primary antibody overnight. Incubation with the secondary antibody was performed for 1 hour. Membranes were reprobed with mouse anti-human $\beta$-actin to ensure equal loading. Protein bands were visualized using an Odyssey Infrared Imaging System (LI-COR Biosciences).

\section{Statistical analysis}

Normality was tested using Kolmogorov-Smirnov. Between groups comparisons for continuous data were performed by Mann-Whitney $U$ test and Kruskal-Wallis with post hoc Dunn's multiple comparison test, where appropriate. All data are expressed as median and range. A two-sided p-value <0.05 was considered statistically significant. Statistical analysis was performed using Prism 5.0 for Windows (Graphpad software, Inc, San Diego, CA). 


\section{Results}

Ovine I-FABP serum and tissue levels increase with gestational age

Serum I-FABP levels were elevated (385 (range, 100 - 1,387) pg/ml) in moderately premature lambs $(n=15)$ when compared to extremely premature lambs $(n=11)$, $156(50-427) \mathrm{pg} / \mathrm{ml} p=0.02$ (Figure 1). Median I-FABP levels tended to be elevated $(255(100-1,872) \mathrm{pg} / \mathrm{ml})$ in the term group $(\mathrm{n}=12)$ when compared

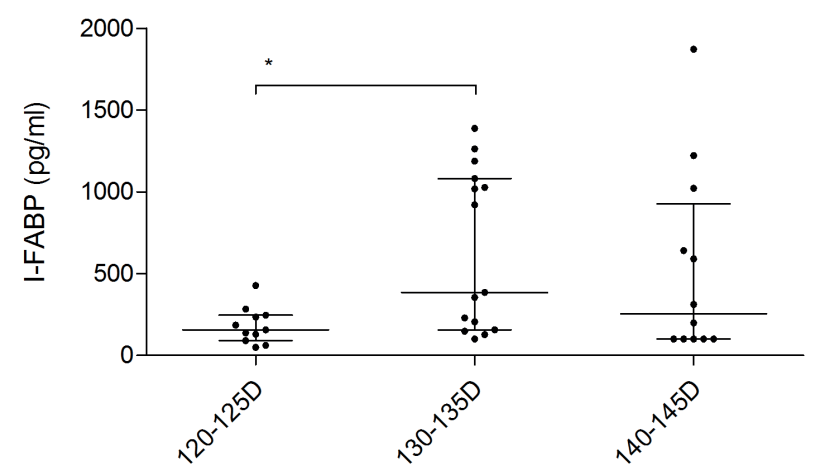

FIGURE 1. Ovine umbilical cord serum I-FABP levels determined by ELISA. I-FABP levels were significantly higher in the 130-135 days gestational age group compared to the 120-125 days group; * $p=0.02$.

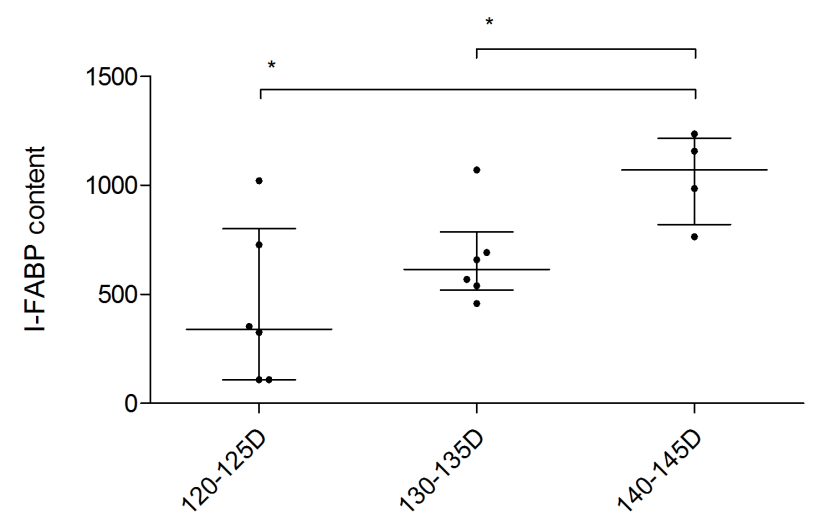

FIGURE 2. Ovine ileal tissue I-FABP content determined by western blot. Relative band intensities (corrected for actin) measured by Odyssey are depicted in the graph. * $p=0.04$. 
with extremely premature lambs $(p=0.06)$. Accordingly, relative high amounts of I-FABP were detected in ileal homogenates of term animals when compared to extremely premature and moderately premature lambs (Figure 2).

TABLE 1. Patient characteristics of post mortem study

\begin{tabular}{lllll}
\hline & Gender & Gestational age & $\begin{array}{l}\text { Time of death (hours } \\
\text { after birth) }\end{array}$ & Presumable cause of death \\
\hline 1 & M & $27^{+5}$ & 24 & Multiple congenital anomalies \\
2 & $\mathrm{~F}$ & $24^{+2}$ & 1.5 & Placental pathology \\
3 & $\mathrm{M}$ & $24^{+5}$ & 1 & Placental pathology \\
4 & $\mathrm{M}$ & $24^{+2}$ & 1.5 & Placental pathology \\
5 & $\mathrm{M}$ & 26 & 0.5 & Meckel Gruber syndrome \\
6 & $\mathrm{~F}$ & 26 & 0.5 & Sacrococcygeal teratoma \\
7 & $\mathrm{M}$ & $29^{+1}$ & 8 & Subcapsular hematoma \\
8 & $\mathrm{M}$ & $30^{+1}$ & 1 & Multiple congenital anomalies \\
9 & $\mathrm{~F}$ & $28^{+3}$ & 24 & Multiple congenital anomalies \\
10 & $\mathrm{M}$ & $29^{+1}$ & 0.5 & Sepsis \\
11 & $\mathrm{~F}$ & $30^{+6}$ & 12 & Cardiac ischemia \\
12 & $\mathrm{M}$ & 30 & 12 & Umbilical cord anomaly \\
13 & $\mathrm{M}$ & $29^{+5}$ & 1 & Multiple congenital anomalies \\
14 & $\mathrm{M}$ & $30^{+4}$ & 2 & Lung hypoplasia \\
15 & $\mathrm{M}$ & 31 & 1 & Cytomegalovirus infection \\
16 & $\mathrm{M}$ & $32^{+6}$ & 24 & Trisomy 21 and leukemia \\
17 & $\mathrm{~F}$ & $37^{+6}$ & 1 & Muscular dystrophy \\
18 & $\mathrm{M}$ & 40 & 0.5 & Muscular dystrophy \\
19 & $\mathrm{M}$ & $40^{+2}$ & 2 & Meconium aspiration \\
20 & $\mathrm{M}$ & 42 & 24 & Placental pathology \\
\hline
\end{tabular}

I-FABP distribution pattern in the ovine ileal epithelium is gestational agedependent

I-FABP expression was most prominent in non-vacuolated enterocytes. In extremely premature lambs, positive cells were only found in the primitive crypts and basis of the villi (Figure $3 A$ ). In the moderately premature group, the crypts and lower parts of the villi were positive for I-FABP (Figure 3B). In the term animals, I-FABP expression was exclusively present in the villus tips (Figure $3 \mathrm{C}$ ). 


\section{I-FABP distribution pattern in human intestine resembles the ovine situation}

The postnatal distribution of I-FABP in extremely preterm, preterm and term born infants was studied in neonates who died within 24 hours after birth. Patient characteristics are summarized in Table 1. As in ovine tissue, the same patterns of enterocyte vacuolization were seen dependent on gestational age. Moreover, I-FABP was predominantly present in non-vacuolated enterocytes. I-FABP expressing cell numbers increased with rising gestational age, comparable with the I-FABP distribution pattern in ovine fetuses (Figure 4).

a

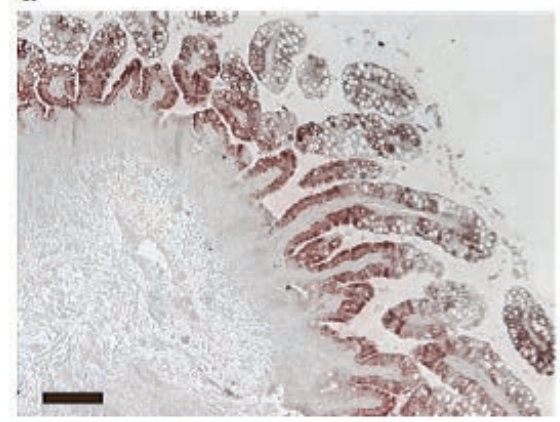

c

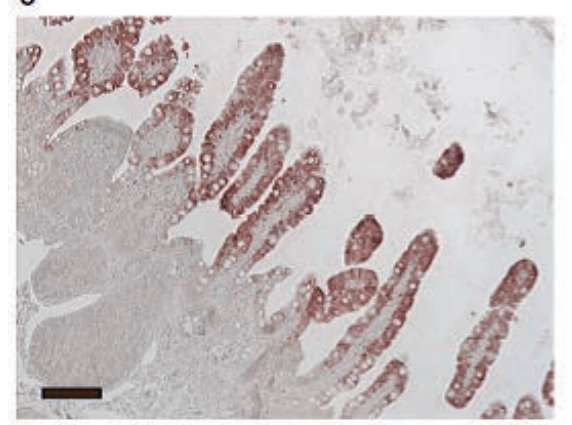

b

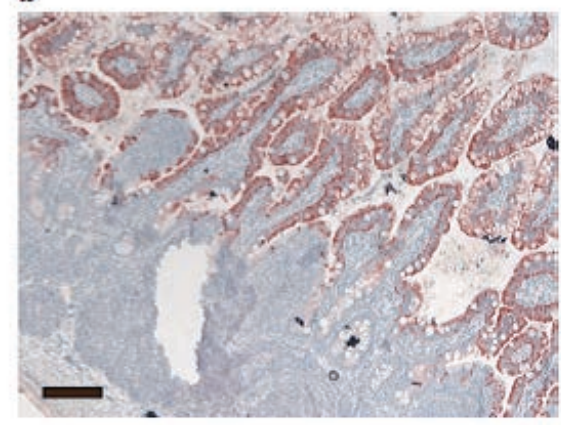

d

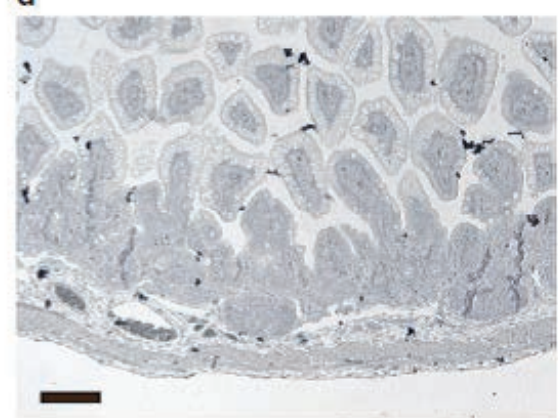

FIGURE 3. Immunohistochemical staining of I-FABP in ovine ileum. A: 120-125 days, B: 130-135 days, C: $140-145$ days, D: negative control. I-FABP expression gradually disappears from the crypts with advancing gestational age, changing into a phenotype characterized by I-FABP expression in the villus tips (magnification 200x). 

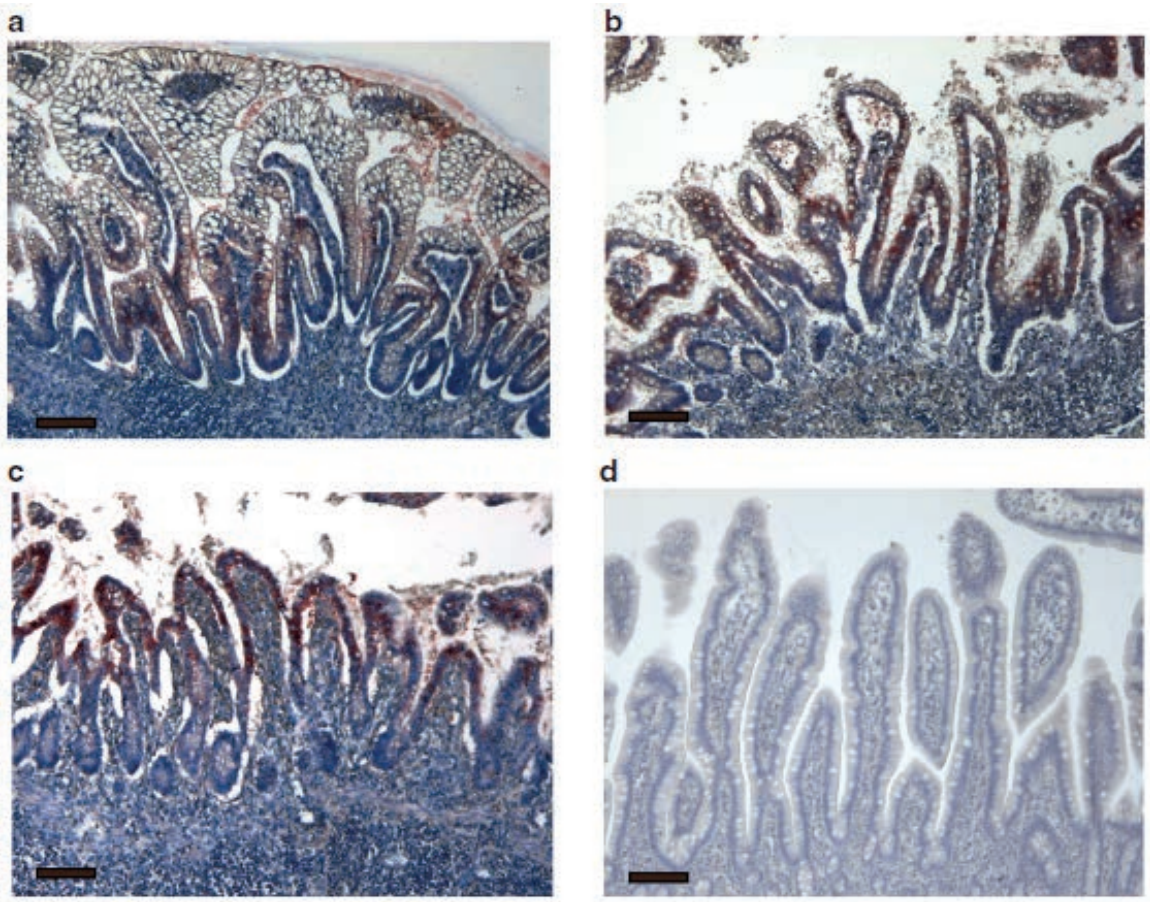

FIGURE 4. Immunohistochemical staining of I-FABP in human ileum. A: <28 weeks, B: 28-37 weeks, C: 37-42 weeks, D: negative control. Paralleling ovine intestinal development, I-FABP expression gradually disappears from the crypts with advancing gestational age, changing into a phenotype characterized by I-FABP expression in the villus tips (magnification 200x).

Epithelial proliferation and apoptosis are not dependent on gestational age in human and ovine intestine

As epithelial I-FABP distribution was dependent on gestational age, the relationship with proliferation and apoptosis was investigated. To determine whether increased I-FABP levels were related with increased epithelial proliferation rates, the proliferative marker Ki-67 was stained. Ki-67 positive cells were primarily located in the crypts of the ileum and to a lesser extent in the villi in all animals (Figure 5). No differences were found between the gestational age groups. Similar to the ovine data, proliferation was not different throughout gestation on human tissue sections as measured by Ki67 (data not shown). 

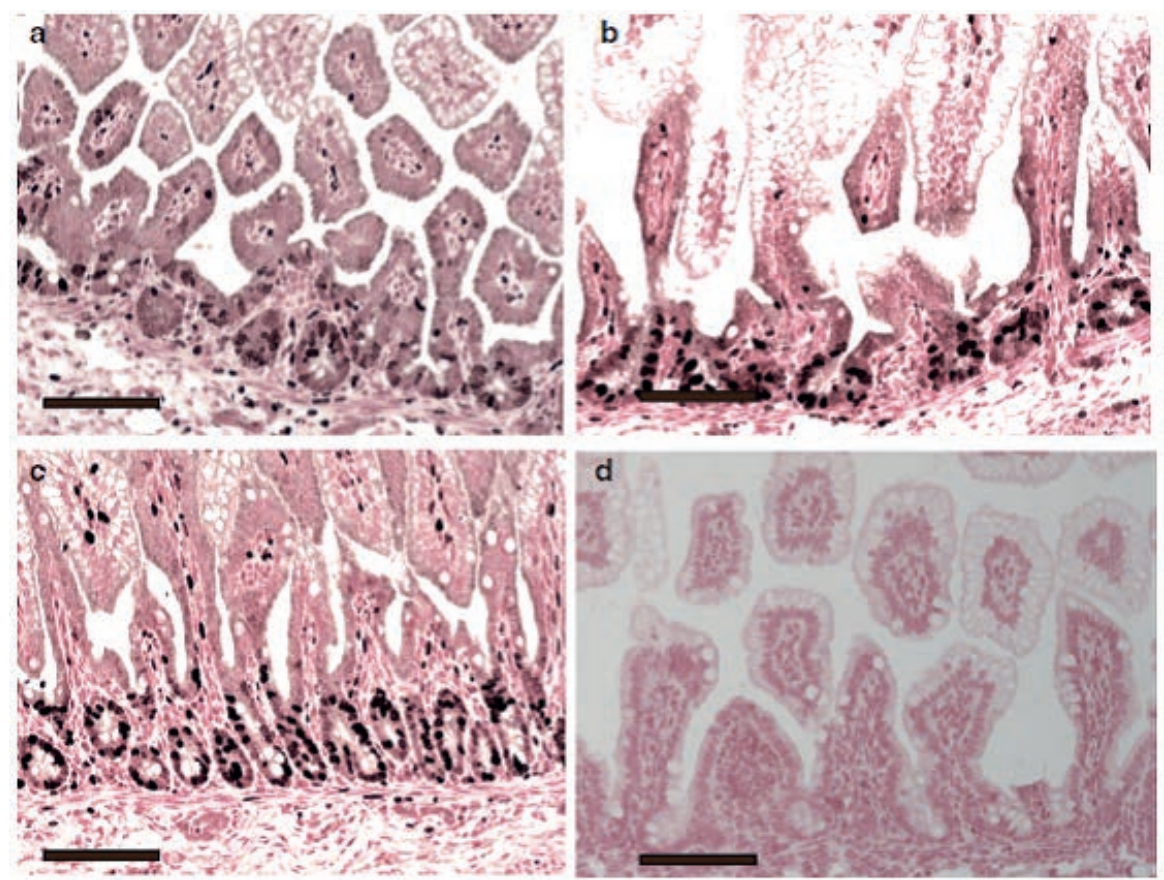

FIGURE 5. Immunohistochemical staining of Ki-67, A: 120-125 days, B: 130-135 days, C: 140-145 days, D: negative control. No differences were found between the different gestational age groups (magnification 200x).

TABLE 2. Baseline characteristics of neonates

\begin{tabular}{|c|c|c|c|c|}
\hline & $\begin{array}{l}\text { Extremely } \\
\text { premature }(n=16)\end{array}$ & $\begin{array}{l}\text { Moderately } \\
\text { premature }(n=18)\end{array}$ & Term $(n=12)$ & P-value \\
\hline $\begin{array}{l}\text { Gestational age } \\
\text { (weeks) }\end{array}$ & $27^{+2}\left(24^{+5}-27^{+6}\right)$ & $34^{+0}\left(28^{+6}-36^{+1}\right)$ & $39^{+4}\left(37^{+1}-42^{+0}\right)$ & $<0.0001$ \\
\hline Birth weight (g) & $928(590-1248)$ & $\begin{array}{l}1683(770- \\
2990)\end{array}$ & $\begin{array}{l}2980(1990- \\
4585)\end{array}$ & $<0.0001$ \\
\hline $\begin{array}{l}\text { Sex (males/ } \\
\text { females) }\end{array}$ & $9 / 7$ & $8 / 10$ & $8 / 4$ & 0.48 \\
\hline $\begin{array}{l}\text { Apgar score } \\
(1 \mathrm{~min})\end{array}$ & $7(1-9)$ & $8(3-9)$ & $8(7-9)$ & 0.01 \\
\hline $\begin{array}{l}\text { Apgar score } \\
(5 \mathrm{~min})\end{array}$ & $8(3-10)$ & $9(5-10)$ & $10(8-10)$ & 0.003 \\
\hline Umbilical vein $\mathrm{pH}$ & $7.30(7.05-7.45)$ & $7.25(6.88-7.41)$ & $7.18(6.97-7.28)$ & 0.07 \\
\hline Chorioamnionitis $^{a}$ & $5 / 8(63 \%)$ & $1 / 10(10 \%)$ & $0 / 1(0 \%)$ & 0.05 \\
\hline
\end{tabular}

Data are presented as median (range)

${ }^{a}$ Cases with histologically proven chorioamnionitis/cases with clinical suspection of chorioamnionitis 
To evaluate whether increased I-FABP levels correlated with decreased epithelial apoptosis rates, immunohistochemical staining for caspase-3 was performed, revealing sparse apoptotic activity in ileal tissue. No differences in caspase-3 presence were seen in gestational age groups (Figure 6).
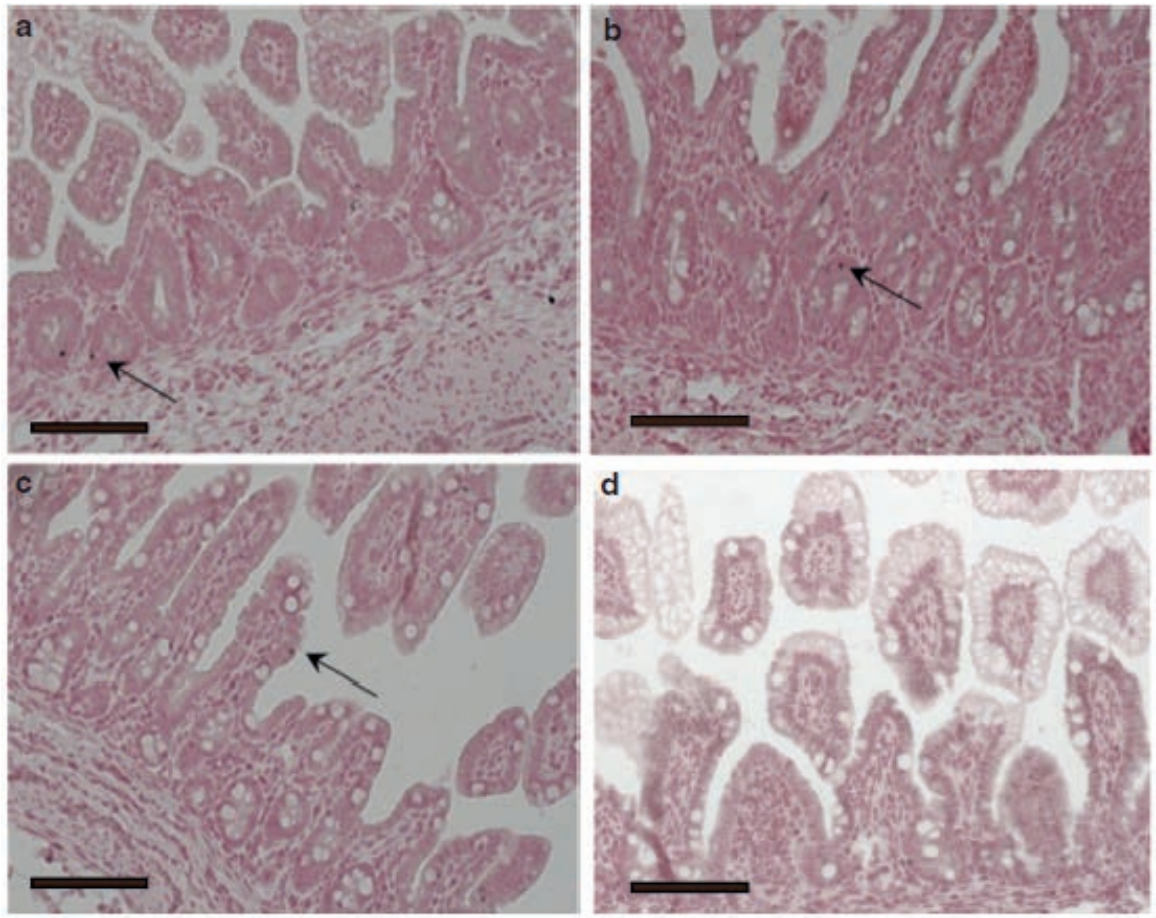

FIGURE 6. Immunohistochemical staining of caspase-3, A: 120-125 days, B: 130-135 days, C: 140145 days, D: negative control. Caspase-3 positive cells are indicated by arrowheads. No differences in caspase-3 expression were found between age groups (magnification 200x).

Human urinary I-FABP levels are elevated in the extremely premature shortly after birth

Thirty-four neonates were included in the current study; 16 in the group with gestational age $<28$ weeks (extremely premature), 18 in the group with gestational age 28-37 weeks (moderately premature), and 12 in the group with gestational age 37-42 weeks (term). Patient characteristics are outlined in Table 2, and clinical parameters are summarized in Table 3. Expectedly, gestational age ad birth weight were significantly different between the groups. Moreover, there was 
TABLE 3. Clinical parameters of the first seven days postpartum

\begin{tabular}{|c|c|c|c|c|}
\hline & $\begin{array}{l}\text { Extremely } \\
\text { premature }(n=16)\end{array}$ & $\begin{array}{l}\text { Moderately } \\
\text { premature } \\
(n=18)\end{array}$ & Term $(n=12)$ & P-value \\
\hline Antibiotic use & $16(100 \%)$ & $9(50 \%)$ & $7(56 \%)$ & 0.004 \\
\hline Indomethacin use & $12(75 \%)$ & $4(22 \%)$ & $0(0 \%)$ & $<0.0001$ \\
\hline Norepinephrine use & $4(25 \%)$ & $0(0 \%)$ & $1(8 \%)$ & 0.06 \\
\hline $\begin{array}{l}\text { Nutrition (breast milk / } \\
\text { formula / combination) }\end{array}$ & $0 / 3 / 13$ & $0 / 7 / 11$ & $1 / 5 / 6$ & 0.33 \\
\hline $\begin{array}{l}\text { Start feeding } \\
\text { (days postnatally) }\end{array}$ & $1(1-3)$ & $0(0-3)$ & $1(0-3)$ & 0.0006 \\
\hline
\end{tabular}

Data are presented as median (range) or total number (percentage)
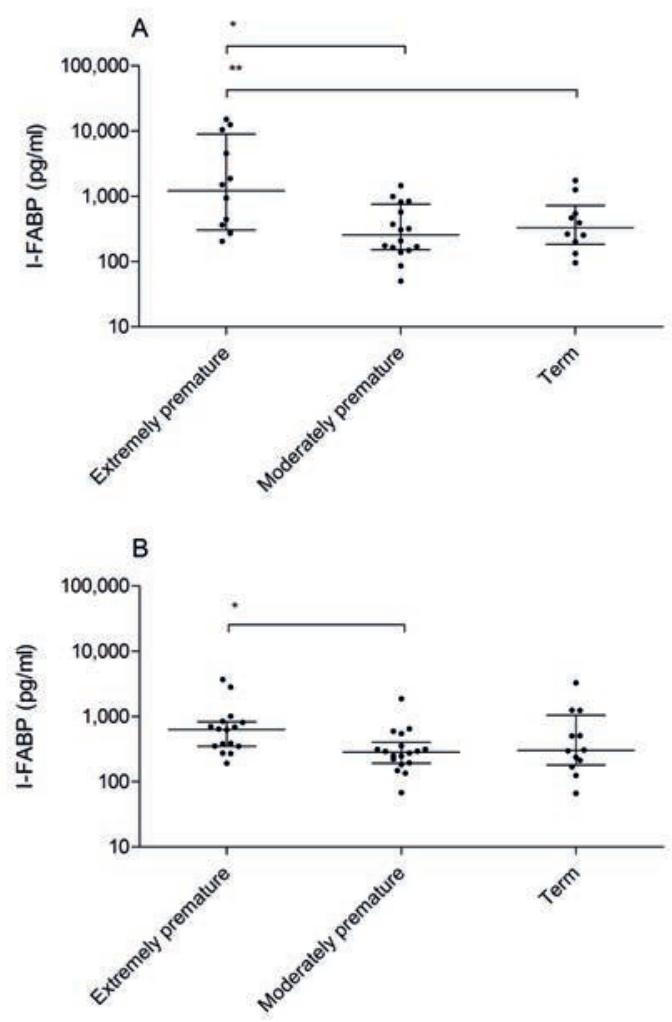

FIGURE 7. Urinary I-FABP determined by ELISA in extremely premature, moderately premature and term neonates. A: I-FABP levels in the first 3 days of life; * $p=0.008$, ** $p=0.04$. B: Urinary I-FABP 4-7 days postpartum; * $\mathrm{p}=0.004$. 
a significant difference between the groups in Apgar scores, histopathologically confirmed chorioamnionitis, postnatal use of antibiotics and NSAIDs, and timing of first enteral feeding.

Median urinary I-FABP levels at 0-3 days postpartum were significantly higher in extremely premature neonates compared with moderately premature neonates and term neonates (1,219 (203 - 15,044) pg/ml, 256 (50 - 1,453) $\mathrm{pg} / \mathrm{ml}(p=0.008), 328(96-1,749) \mathrm{pg} / \mathrm{ml}(p=0.04))$, Figure 7A. This difference was still present at 4-7 days postpartum between extremely premature neonates (631 (191 - 3,670) pg/ml) and moderately premature neonates (282 (67 $1,863) \mathrm{pg} / \mathrm{ml}, p=0.004)$, but not between extremely premature neonates and term neonates (302 (66 - 3,261), $p=0.16)$, Figure 7B. From 0-3 days to 4-7 days postpartum, I-FABP levels decreased significantly $(p=0.04)$, with median $24 \%$. However, this difference was obviously caused by a decrease of urinary I-FABP levels in the extremely premature group (median decrease 64\%, $p=0.04$ ), while in the moderately premature and term group, urinary I-FABP levels did not decrease significantly, $p=0.79$ and $p=0.45$, respectively.

TABLE 4. Eligibility and inclusion numbers of neonates

\begin{tabular}{llll}
\hline & Extremely premature & Moderately premature & Term \\
\hline Eligible during study period & 22 & 129 & 46 \\
Approached for consent & 22 & 25 & 25 \\
Number included & 16 & 18 & 12 \\
\hline
\end{tabular}

\section{Discussion}

This study shows that the intestinal distribution pattern of I-FABP is gestational age dependent in both sheep and humans. In the ovine gut, I-FABP levels increase during gestation, which is also reflected by I-FABP levels in umbilical cord serum upon delivery. The epithelial distribution pattern changes from a phenotype displaying I-FABP positive enterocytes merely in the crypts at 120-125 days of gestation to a phenotype with I-FABP expressing cells restricted to the villus tips in term lambs. In human intestine, similar changes of epithelial maturation during gestation were seen. However, the human urinary I-FABP levels during the first three days after birth were inconsistent with ovine data and human in utero data.

The differences may be explained by the following aspects. First, the fetal gut contains immature vacuolated enterocytes, which decrease in number through gestation, as observed in the current study and by others ${ }^{15,18}$. After birth, vacuolated 
enterocytes rapidly mature, resulting in high enterocyte turnover rates and I-FABP release. As the extremely premature gut contains more immature enterocytes, turnover rates can be higher compared to the less premature intestine. This seems however unlikely as the current data do not show differences between gestational age groups in mucosal proliferation rates in the first days after birth. Second, the stimuli of bacterial colonization after birth may lead to an inflammatory response, leading to damage of immature enterocytes due to inadequate immune response and barrier function associated with prematurity ${ }^{19}$. Elevated I-FABP levels may even underestimate the amount of enterocyte damage. Early intestinal damage affects primarily the villus tips. In extreme preterm infants, such mucosal injury may not lead to a significant increase in serum and urinary I-FABP levels since the protein is primarily expressed by epithelial cells lining the crypts and lower half of the villi. Reference values in clinical practice should therefore be adapted according to gestational age. In this context, the number of days after birth should be taken into account. Third, in the ovine model lambs are born prematurely because of human intervention. In humans however, preterm labor is frequently caused by chorioamnionitis ${ }^{20}$. Incidence of chorioamnionitis was indeed increased in extremely premature neonates in this cohort. Previous studies have shown that chorioamnionitis can cause intestinal damage in utero ${ }^{21-23}$. It is therefore likely that premature born neonates have already suffered intestinal damage in utero, reflected by high urinary I-FABP levels directly after birth. Other confounding factors such as indomethacin use may also increase I-FABP levels in premature neonates after birth. Finally, differences in renal function might contribute to antenatal and postnatal I-FABP levels. However, due to its small molecular size (14 kDa), plasma and urinary I-FABP levels are hardly affected by renal function. In a recent study, we have shown that differences in urinary I-FABP levels are not affected when corrected for creatinine even in severely ill neonates ${ }^{24}$.

The data in this study have important implications. As intestinal and circulatory I-FABP levels increase with gestational age, I-FABP reflects gut maturation, which is underlined by the histological change of phenotype. This makes I-FABP a potentially suitable marker for intestinal maturation, at least in the sterile, healthy intestine. Markers for intestinal maturation are currently lacking, and may be of great value for guiding feeding strategies. It is shown that standardized feeding protocols result in earlier successful enteral feeding without increased rates of major morbidities ${ }^{25,26}$. A clinical study is needed to investigate whether implementation of I-FABP measurements in such a protocol is beneficial, for 
instance with slower feeding advancements in patients with low perinatal I-FABP levels reflecting gut immaturity. However, it should be noted that urinary I-FABP levels within the first three days after birth are not suitable for this purpose, since these levels do not reflect the in utero situation, possibly due to confounding factors such as bacterial colonization, chorioamnionitis, and indomethacin use. It may therefore be more adequate to measure perinatal I-FABP levels in cord blood, which is acquired routinely in most in-hospital deliveries. In the current study, this was impossible due to its retrospective design, however the ovine cord blood data underline this hypothesis. In the future, it should be investigated whether human cord blood I-FABP levels correlate with the development of gastrointestinal morbidity, such as NEC and feeding intolerance.

In this study, neonates with intestinal disease were excluded to avoid confounding effects on I-FABP levels. It is appealing to investigate whether (extremely) premature neonates with high I-FABP levels shortly after birth are at increased risk for developing intestinal disorders, or whether these high levels normalize without an increased risk. I-FABP as a screening tool for NEC has been investigated before ${ }^{27}$. Urinary I-FABP levels in the first 90 hours after birth were measured in 55 preterm neonates. In this group, 9 infants developed NEC, all of them having elevated I-FABP values. Sixteen other neonates also had high I-FABP values but did not develop NEC. Interestingly, all 16 patients had significantly more feeding problems compared to the infants with normal I-FABP values. Future studies could address the effectiveness of preventive strategies in premature neonates with high I-FABP levels.

In conclusion, I-FABP distribution is maturation dependent both in ovine and human gut. Increasing gestational age parallels an increase in I-FABP tissue content and circulating I-FABP levels in sheep, making I-FABP a promising marker of gut maturation. However, this study shows that extremely preterm neonates suffer from more intestinal damage early in life than late preterm infants, indicating that the effect of maturation is confounded by postnatal factors. Urinary I-FABP levels in the first days after birth are therefore not suitable to assess gut maturation. Future research should evaluate whether I-FABP levels in human cord blood accurately measure gut maturation. 


\section{References}

1. Neu J. Gastrointestinal development and meeting the nutritional needs of premature infants. Am J Clin Nutr 2007;85:629S-634S.

2. Rouwet EV, Heineman E, Buurman WA, et al. Intestinal permeability and carriermediated monosaccharide absorption in preterm neonates during the early postnatal period. Pediatr Res 2002;51:64-70.

3. Neu J, Chen M, Beierle E. Intestinal innate immunity: how does it relate to the pathogenesis of necrotizing enterocolitis. Semin Pediatr Surg 2005;14:137-44.

4. Lin PW, Nasr TR, Stoll BJ. Necrotizing enterocolitis: recent scientific advances in pathophysiology and prevention. Semin Perinatol 2008;32:70-82.

5. Kanda T, Fujii H, Tani T, et al. Intestinal fatty acid-binding protein is a useful diagnostic marker for mesenteric infarction in humans. Gastroenterology 1996;110:339-43.

6. Thuijls G, Derikx JP, van Wijck K, et al. Non-invasive markers for early diagnosis and determination of the severity of necrotizing enterocolitis. Ann Surg 2010;251:1174-80.

7. Derikx JP, Evennett NJ, Degraeuwe PL, et al. Urine based detection of intestinal mucosal cell damage in neonates with suspected necrotising enterocolitis. Gut 2007;56:14735.

8. van de Poll MC, Derikx JP, Buurman WA, et al. Liver manipulation causes hepatocyte injury and precedes systemic inflammation in patients undergoing liver resection. World J Surg 2007;31:2033-8.

9. Ng EW, Poon TC, Lam HS, et al. Gut-Associated Biomarkers L-FABP, I-FABP, and TFF3 and LIT Score for Diagnosis of Surgical Necrotizing Enterocolitis in Preterm Infants. Ann Surg 2013;258:1111-8.

10. Adriaanse MP, Tack GJ, Passos VL, et al. Serum I-FABP as marker for enterocyte damage in coeliac disease and its relation to villous atrophy and circulating autoantibodies. Aliment Pharmacol Ther 2013;37:482-90.

11. Derikx JP, Blijlevens NM, Donnelly JP, et al. Loss of enterocyte mass is accompanied by diminished turnover of enterocytes after myeloablative therapy in haematopoietic stem-cell transplant recipients. Ann Oncol 2009;20:337-42.

12. Veerkamp JH, Paulussen RJ, Peeters RA, et al. Detection, tissue distribution and (sub) cellular localization of fatty acid-binding protein types. Mol Cell Biochem 1990;98:118.

13. Trahair J, Robinson P. The development of the ovine small intestine. Anat Rec 1986;214:294-303.

14. Asari M, Kawaguchi N, Wakui S, et al. Development of the bovine ileal mucosa. Acta Anat (Basel) 1987;129:315-24.

15. Moxey PC, Trier JS. Specialized cell types in the human fetal small intestine. Anat Rec 1978;191:269-85.

16. Skrzypek T, Valverde Piedra JL, Skrzypek H, et al. Gradual disappearance of vacuolated enterocytes in the small intestine of neonatal piglets. J Physiol Pharmacol 2007;58 Suppl 3:87-95. 
17. Wolfs TG, Jellema RK, Turrisi G, et al. Inflammation-induced immune suppression of the fetus: a potential link between chorioamnionitis and postnatal early onset sepsis. J Matern Fetal Neonatal Med 2012;25 Suppl 1:8-11.

18. Trahair JF, Wilson JM, Neutra MR. Identification of a marker antigen for the endocytic stage of intestinal development in rat, sheep, and human. J Pediatr Gastroenterol Nutr 1995;21:277-87.

19. Adkins B, Leclerc C, Marshall-Clarke S. Neonatal adaptive immunity comes of age. Nat Rev Immunol 2004;4:553-64.

20. Goldenberg RL, Hauth JC, Andrews WW. Intrauterine infection and preterm delivery. N Engl J Med 2000;342:1500-7.

21. Been JV, Lievense S, Zimmermann $\sqcup$, et al. Chorioamnionitis as a risk factor for necrotizing enterocolitis: a systematic review and meta-analysis. J Pediatr 2013;162:236-42 e2.

22. Wolfs TG, Buurman WA, Zoer B, et al. Endotoxin induced chorioamnionitis prevents intestinal development during gestation in fetal sheep. PLoS One 2009;4:e5837.

23. Wolfs TG, Kallapur SG, Knox CL, et al. Antenatal ureaplasma infection impairs development of the fetal ovine gut in an IL-1-dependent manner. Mucosal Immunol 2013;6:547-56.

24. Reisinger KW, Derikx JP, Thuijls G, et al. Noninvasive measurement of intestinal epithelial damage at time of refeeding can predict clinical outcome after necrotizing enterocolitis. Pediatr Res 2013;73:209-13.

25. Kuzma-O'Reilly B, Duenas ML, Greecher C, et al. Evaluation, development, and implementation of potentially better practices in neonatal intensive care nutrition. Pediatrics 2003;111:e461-70.

26. McCallie KR, Lee HC, Mayer O, et al. Improved outcomes with a standardized feeding protocol for very low birth weight infants. J Perinatol 2011;31 Suppl 1:S61-7.

27. Mannoia K, Boskovic DS, Slater L, et al. Necrotizing enterocolitis is associated with neonatal intestinal injury. J Pediatr Surg 2011;46:81-5. 


Chapter 9. Breast feeding improves gut maturation compared to formula feeding in preterm babies

Kostan W. Reisinger, Loes de Vaan, Boris W. Kramer, Tim G.A.M. Wolfs, L.W. Ernest van Heurn, Joep P.M. Derikx. Journal of Pediatric Gastroenterology and Nutrition 2014. 59(6):720-4 


\begin{abstract}
Objective

The incidence of necrotizing enterocolitis (NEC) is higher in formula fed babies than in breast fed babies, which may be caused by breastfeeding induced gut maturation. The effect of breastfeeding on gut maturation has been widely studied in animal models. This study aimed to assess the effects of breastfeeding on intestinal maturation in prematurely born babies by evaluating postnatal changes in urinary intestinal Fatty Acid Binding Protein (I-FABP) levels, a specific enterocyte marker.
\end{abstract}

\title{
Methods
}

Gut maturation in forty premature babies ( $<37$ weeks of gestation) without gastrointestinal morbidity was studied, of whom 21 were exclusively breast fed and 19 were formula fed. Urinary I-FABP levels as measure of gut maturation were measured at 5, 12, 19 and 26 days after birth.

\section{Results}

In breast fed infants, there was a significant increase in median urinary I-FABP levels between 5 and 12 days after birth (104 [78 - 340] pg/mL to 408 [173 - 1,028] $\mathrm{pg} / \mathrm{mL}, p=0.002)$, while I-FABP concentration in formula fed infants increased between 12 and 19 days after birth (105 [44 - 557] pg/mL, 723 [103 - 1,670] pg/mL, $p=0.004)$. Breast fed babies had significantly higher median urinary I-FABP levels at postnatal day $12(p=0.01)$.

\section{Conclusion}

The time course of the postnatal increase in urinary I-FABP levels reflecting gut maturation was significantly delayed in formula fed babies, suggesting a delayed physiological response in formula fed compared to breast fed infants. 


\section{Introduction}

Necrotizing enterocolitis (NEC) is the most common gastrointestinal emergency in the neonatal intensive care unit (NICU). The prevalence of NEC is largely related to birth weight and gestational age, with approximately one in ten very low birth weight (VLBW) infants (<1,500 gram) developing NEC ${ }^{1}$. Currently, an excessive inflammatory response against commensal bacteria by the immature intestine following mucosal injury in the postnatal period is the leading hypothesis in the pathophysiology of NEC ${ }^{2,3}$. Since there is an estimated three-fold to ten-fold risk reduction in infants fed with breast milk compared with those fed formula milk ${ }^{4-8}$, it can be hypothesized that breast feeding is protective against NEC development through improved gut maturation. Furthermore, animal research has shown formula related mucosal atrophy, dysfunction and subsequent elevated NEC risk ${ }^{9}$.

Intestinal Fatty Acid Binding Protein (I-FABP) is a small (14 - $15 \mathrm{kDa}$ ) cytosolic protein, exclusively present in mature enterocytes of the small intestine and colon ${ }^{10,11}$. It is released into the circulation when enterocytes detach from villi at the end of their lifespan ${ }^{12,13}$. We have previously shown that I-FABP reflects gut maturation in utero ${ }^{14}$. I-FABP passes the glomerular filter and can easily be detected in urine (plasma half-life \pm 11 minutes).

The effects of breast milk on intestinal growth have principally been studied in vitro and in animal experiments ${ }^{15}$. This study aims to evaluate the effects of breastfeeding compared with formula feeding on human intestinal maturation in prematurely born babies in vivo by evaluating changes in urinary I-FABP levels during the first weeks after birth.

\section{Methods}

\section{Patient inclusion}

All babies admitted to the neonatal intensive care unit (NICU) of the Maastricht University Medical Center between July 2007 and July 2008 were eligible for participation. These patients were enrolled in a prospectively maintained database for the study of factors associated with NEC, with regular collection of urine and stool. Written informed consent was obtained from both parents or caretakers, and the study was conducted with approval from local medical ethical committees and according to the declaration of Helsinki. The principles of good clinical practice (GCP) were followed during this study. Infants were included if they met the following inclusion criteria: 1) $<37$ weeks of gestation, 
2) first enteral feeding within 4 days after birth, and 3) diet consisting of either exclusively breast milk or exclusively formula milk. The only exclusion criterion was development of significant gastrointestinal pathology during the 30-day study period, defined as disease of the gastrointestinal tract necessitating surgery, antibiotic treatment, cardiopulmonary support, or discontinuation or reduction of enteral feeding. Initiation of feeding and advancement of feeding volumes were realized according to local protocol. The standard guidelines consisted of initiation of oral feeding at day 1-4 after birth. Depending on the infant's gestational age and general condition, the feeding volume was increased with $10-20 \mathrm{~mL} / \mathrm{kg} /$ day and discontinued if there were signs of feeding intolerance including bilious gastric retentions, abdominal distention, emesis or bloody stools.

Differences in intestinal inflammation between breast fed infants and formula fed infants was assessed by the fecal inflammatory marker calprotectin in the first stool sample available.

\section{Sample collection and I-FABP measurements}

Only samples collected on the fifth postnatal day and further were included in the analyses as enteral feeding was initiated in all infants by that time, as defined in the inclusion criteria. Urine samples collected on the fifth, $12^{\text {th }}, 19^{\text {th }}$, and $26^{\text {th }}$ day after birth were included in the current study. Samples were obtained by placement of a dental cotton roll in the diaper of the neonate. Once saturated with urine, the role was placed in a sterile $5 \mathrm{~mL}$ syringe from which urine was consequently pressed into Micronic tubes. Samples were stored at $-20^{\circ} \mathrm{C}$ until analysis. First available stool samples were collected and stored immediately at $-20^{\circ} \mathrm{C}$ until batch analysis.

After patient inclusion was completed, urinary I-FABP concentrations were measured using an in-house enzyme-linked immunosorbent assay (ELISA) selectively detecting human I-FABP (lower detection limit: $12.5 \mathrm{pg} / \mathrm{mL}$ ). Values are expressed in $\mathrm{pg} / \mathrm{mL}$. Uncorrected urinary I-FABP levels correlate well with urinary I-FABP levels corrected for urine concentration ${ }^{16}$. 


\section{Fecal calprotectin measurement}

Fecal calprotectin was measured to assess intestinal inflammatory differences between formula feeding and breast feeding. Calprotectin is a heterodimeric peptide $(36 \mathrm{kDa})$, which is released from the cytosol of neutrophils upon activation. Fecal calprotectin is a specific marker for neutrophil infiltrate in bowel mucosa. In intestinal inflammation, calprotectin is readily detectable in feces and plasma ${ }^{17}$.

After thawing of feces, $100 \mathrm{mg}$ was weighed and $4.9 \mathrm{ml}$ extraction buffer $\left(0.1 \mathrm{M}\right.$ Tris, $0.15 \mathrm{M} \mathrm{NaCl}, 1.0 \mathrm{M}$ urea, $10 \mathrm{mM} \mathrm{CaCl} \cdot 2 \mathrm{H}_{2} \mathrm{O}, 0.1 \mathrm{M}$ citric acid, $0.5 \%$ $\mathrm{BSA}, \mathrm{pH}$ 8.0) was added ${ }^{18}$. After 30 minutes shaking, $1 \mathrm{ml}$ of suspension was centrifuged at $10,000 \mathrm{rpm}$ for 20 minutes at $4^{\circ} \mathrm{C}$ and supernatant was aliquoted and stored at $-20^{\circ} \mathrm{C}$. Calprotectin concentration was measured in lysate using the commercially available calprotectin ELISA (lower detection limit $625 \mathrm{ng} / \mathrm{ml}$, Hycult Biotechnology, Uden, the Netherlands). Fecal calprotectin concentration is given in $\mu g$ calprotectin per gram feces.

\section{Statistical analysis}

Statistical analyses were performed utilizing Prism 5.0 for Windows (GraphPad Software Inc. San Diego, CA) and SPSS 20.0 for Windows (SPSS Inc. Chicago, IL). The Kolmogorov-Smirnov test was used to assess for normal distribution. MannWhitney $\mathrm{U}$ test was used for between group comparisons of continuous data. Friedman test was used for repeated measures. Dichotomous variables were compared using Fisher exact test. Receiver-operator curve (ROC) analysis was performed to differentiate formula fed infants from breast fed infants. All data are presented as median and interquartile range (IQR). Significance was defined $a$ priori as $p<0.05$ for all tests. The a priori sample size calculation was based on the intestinal permeability difference between formula and breast fed babies at day 7 (median lactulose/mannitol ratio 0.205 versus 0.076 , respectively) ${ }^{19}$. Calculation with $\alpha=0.05$ and $1-\beta=0.80$ resulted in 20 subjects per group.

\section{Results}

\section{Patients}

A total of 40 patients were enrolled in the study, of which 21 were exclusively breast fed and 19 were exclusively formula fed. Figure 1 shows the flow diagram of enrollment. Median gestational age was $30^{+1}\left[\mathrm{IQR}, 28^{+2}-33^{+0}\right]$ days and 
median birth weight was 1,400 [1,080 - 1,740] grams. Both groups contained 10 male subjects. Human milk based breast milk fortifier was administered in 11 out of 21 breast fed infants, on median day 16, i.e. between the second and third study time-points. There were no significant differences in gestational age, birth weight or sex between the two groups, however there was a trend of lower median gestational age in the breast fed group (Table 1). Median NICU length of

\section{Flow Diagram of Enrollment}

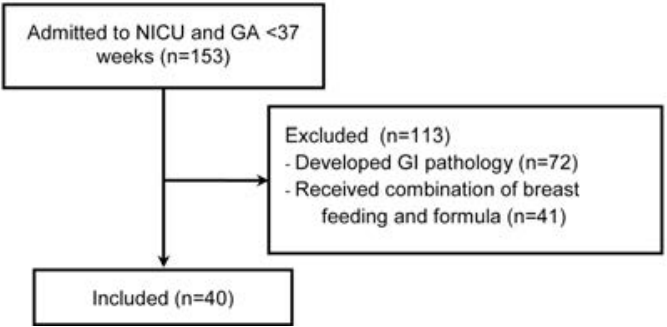

FIGURE 1. Flow diagram of enrollment.

admittance was 20 days, with no significant difference between the two groups. Prematurity was the primary reason for admittance to the NICU in all babies. Comorbid conditions are given in Table 2. Some subjects were lost to follow-up at 19 days after birth $(n=14$, of which 10 were transferred to the referring hospital and 4 to the medium care unit, all because of clinical improvement) and at 26 days after birth ( $n=12$, of which 7 were transferred to the referring hospital and 5 to the medium care unit, all because of clinical improvement). Fecal calprotectin levels in first available stool samples were not significantly different between breast fed infants (42.3 [31.2 - 195.1] $\mathrm{\mu g} / \mathrm{g}$ feces) and formula fed infants (87.6 [31.2 466.4] $\mu \mathrm{g} / \mathrm{g}$ feces, $p=0.23)$, signifying no difference in intestinal inflammation between groups.

TABLE 1. Patient characteristics

\begin{tabular}{lllr}
\hline & Breast fed $(\mathrm{N}=21)$ & Formula fed $(\mathrm{N}=19)$ & $\mathrm{P}$ \\
\hline Gender & $10 \mathrm{M}(45 \%)$ & $10 \mathrm{M}(50 \%)$ & 0.78 \\
Birth weight (grams) & $1,262(1,044-1,499) 1,648(1,168-1,934) 0.11$ \\
Gestational age (weeks) & $29^{+3}\left(27^{+5}-30^{+5}\right)$ & $31^{+3}\left(29^{+0}-34^{+1}\right)$ & 0.06 \\
Duration of NICU admittance (days) & $27(16-43)$ & $18(14-29)$ & 0.21 \\
\hline
\end{tabular}

Data are expressed as total number (percentage) or median (interquartile range) 


\section{Urinary I-FABP concentrations in breast fed vs. formula fed babies}

Median I-FABP levels for the breast fed group were 104 [78 - 340] pg/mL, 408 [173 - 1,028] pg/mL, 374 [153 - 1,404] pg/mL and 634 [243 - 2,350] pg/ $\mathrm{mL}$ at 5, 12, 19 and 26 days after birth, respectively. Median I-FABP levels in formula fed babies were 152 [79 - 348] pg/mL, 105 [44 - 557] pg/mL, 723 [103 - 1,670] pg/mL and 659 [241 - 3,451] pg/mL at 5, 12, 19 and 26 days

TABLE 2. Co-morbidities

\begin{tabular}{|c|c|c|c|c|}
\hline & & Breast fed & Formula fed & $\mathbf{P}$ \\
\hline \multicolumn{2}{|c|}{$\begin{array}{l}\text { Cardiac/hemodynamic ASD/VSD } \\
\text { complications }\end{array}$} & $2(9.5)$ & $3(15.7)$ & 0.65 \\
\hline & PDA & $3(14.3)$ & $1(5.3)$ & 0.61 \\
\hline \multirow{5}{*}{$\begin{array}{l}\text { Pulmonary } \\
\text { complications }\end{array}$} & IRDS & $12(57)$ & $4(21)$ & 0.03 \\
\hline & Pneumothorax & $1(4.7)$ & $1(5.3)$ & 1.00 \\
\hline & PPHN & $1(4.7)$ & $0(0)$ & 1.00 \\
\hline & BPD & $1(4.7)$ & $2(10.5)$ & 0.60 \\
\hline & Lung emphysema & $0(0)$ & $1(5.3)$ & 0.48 \\
\hline \multirow[t]{2}{*}{ Infectious } & Sepsis & $11(52.4)$ & $7(36.8)$ & 0.36 \\
\hline & Perinatal infection & $2(9.5)$ & $1(5.3)$ & 1.00 \\
\hline Metabolic & Hyperbilirubinemia & $12(57.1)$ & $15(79)$ & 0.19 \\
\hline \multirow[t]{5}{*}{ Neurological } & Plexus choroideus cyst & $1(4.7)$ & $0(0)$ & 1.00 \\
\hline & Hydrocephalus & $0(0)$ & $1(5.3)$ & 0.48 \\
\hline & IVH & $4(19)$ & $2(10.5)$ & 0.66 \\
\hline & $\begin{array}{l}\text { Subependymal } \\
\text { hemorrhage }\end{array}$ & $0(0)$ & $1(5.3)$ & 0.48 \\
\hline & Cerebral abscesses & $0(0)$ & $2(10.5)$ & 0.22 \\
\hline Other & Inguinal hernia & $1(4.7)$ & $1(5.3)$ & 1.00 \\
\hline
\end{tabular}

Data are expressed as number (\%)

ASD: atrial septal defect; IRDS: infant respiratory distress syndrome; PDA: persistent ductus arteriosus; IVH: interventricular hemorrhage; BPD: bronchopulmonary dysplasia;

PPHN: persistent pulmonary hypertension of the newborn

after birth, respectively. Both groups showed a significant increase in urinary I-FABP concentrations in the first weeks after birth. For the breast fed infants this increase in I-FABP levels was observed between 5 and 12 days after birth (104 [78 - 340] pg/mL to 408 [173 - 1,028] pg/mL, $p=0.002)$, while I-FABP levels in formula fed infants increased significantly between 12 and 19 days after birth (105 [44 - 557] pg/mL to 723 [103 - 1,670] pg/mL, $p=0.004)$. As a consequence, infants that were exclusively formula fed had significantly lower median I-FABP 


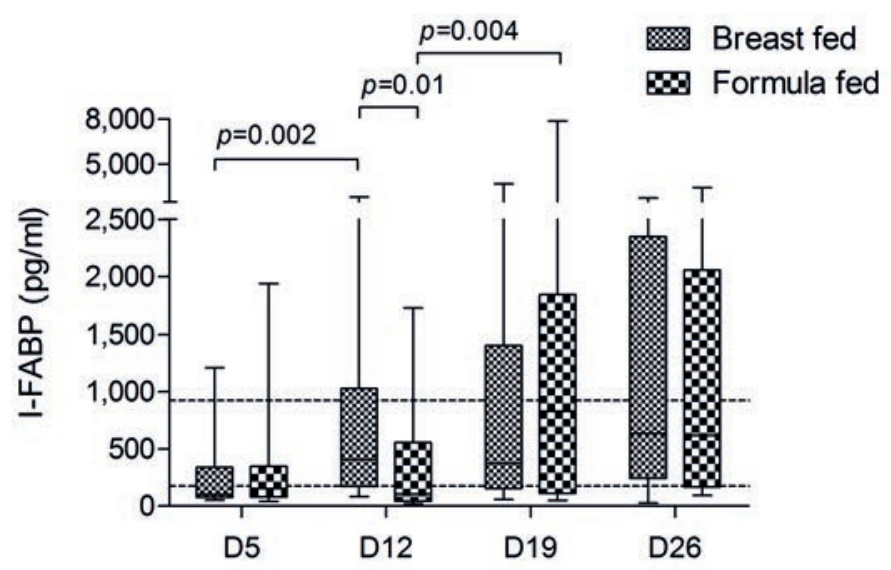

FIGURE 2. Urinary I-FABP levels in breast fed versus formula fed babies at postnatal day 5, 12, 19 and 26. Bars represent median I-FABP levels with interquartile ranges. Dotted lines represent the normality range $(174-924 \mathrm{pg} / \mathrm{mL})$ for babies $26-36$ weeks of gestational age ${ }^{16}$.

levels at postnatal day $12(p=0.01)$. ROC plotting produced an optimal cut-off point of $77.6 \mathrm{pg} / \mathrm{mL}$ to differentiate formula fed from breast fed infants at day 12 , with sensitivity $42 \%$ and specificity $100 \%$, and area under the curve 0.74 (95\% confidence interval, 0.58-0.90). There were no significant differences in median I-FABP levels between the two groups at postnatal days 5 and 19. Group sizes at day 26 were too low for statistical comparison, as 26 out of 40 patients had dropped out of the study at this time-point. I-FABP levels at different time points are shown in Figure 2.

Total volume of enteral feeding was recorded every day to investigate whether type of feeding correlated with feeding tolerance. Volume of enteral intake (mL/ kg body weight) did not differ significantly between the two groups during the studied period ( $p=0.93$, Figure 3 ). There were no differences in other signs of feeding intolerance, defined as episodes of discontinuation of enteral feeding or frequency and cumulative amount of gastric retentions (Table 3).

\section{Discussion}

The results of this study show a significant delay in the postnatal increase of I-FABP levels in formula fed premature babies compared to breast fed premature babies, suggesting a delayed physiological development of intestinal mucosa in formula fed prematures. I-FABP enters the circulation when enterocytes detach 


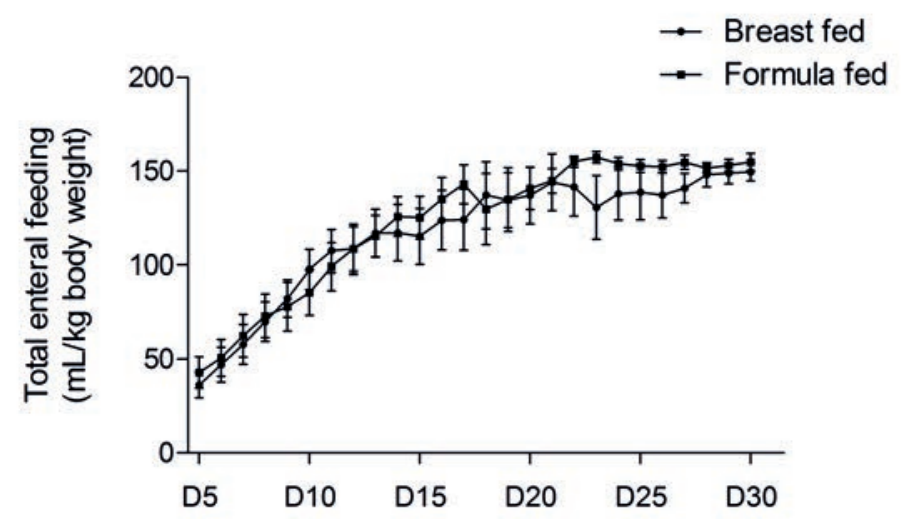

FIGURE 3. Enteral feeding in $\mathrm{mL}$ per $\mathrm{kg}$ body weight. There were no significant differences in volume or expansion of enteral feeding between breast fed and formula fed babies in the first 30 days after birth.

from their villi at the end of their lifespan and upon intestinal cell damage ${ }^{12}$, 13. Therefore infants with possible intestinal disease were excluded from the study as high I-FABP levels in these children may obscure the I-FABP release from detaching villi during normal maturation. This was confirmed by low levels of fecal calprotectin in the first stool sample available. We have shown before that I-FABP correlates with gut maturation in utero ${ }^{14}$. I-FABP levels within normal ranges TABLE 3. Clinical signs of feeding intolerance

\begin{tabular}{|c|c|c|c|}
\hline & Breast fed $(\mathrm{N}=21)$ & Formula fed $(\mathrm{N}=19)$ & $\mathbf{P}$ \\
\hline $\begin{array}{l}\text { Episode of discontinuated } \\
\text { enteral feeding }\end{array}$ & $2(10 \%)^{1}$ & $2(11 \%)^{1}$ & 0.92 \\
\hline Episode of gastric retentions & $20(95 \%)^{1}$ & $18(95 \%)^{1}$ & 0.94 \\
\hline Days with gastric retentions & $6(2-9)$ & $4(2-7)$ & 0.34 \\
\hline $\begin{array}{l}\text { Cumulative amount of gastric } \\
\text { retentions }(\mathrm{mL})\end{array}$ & $30(9-45)$ & $21(7-40)$ & 0.35 \\
\hline
\end{tabular}

${ }^{1}$ Number of babies experiencing at least one episode during study period
Data are expressed as total number (percentage) or median (interquartile range)

represent the continuous release of I-FABP from mature enterocytes detaching into the gut lumen ${ }^{10}$. The delay in the increase towards normal values of I-FABP in formula fed premature babies may therefore indicate decreased maturation of enterocytes, as normal values were measured at postnatal day 19 compared to 
day 12 in breast fed babies. We have previously reported mean normal values of I-FABP ranging from $100-200 \mathrm{pg} / \mathrm{mL}$ in healthy children and adults ${ }^{20-23}$ and 434 $\mathrm{pg} / \mathrm{mL}$ in premature babies ${ }^{16}$.

This is the first study to assess I-FABP levels in breast fed versus formula fed prematures. In other studies, intestinal permeability measured by lactulose to mannitol $(\mathrm{L} / \mathrm{M})$ ratio, has been used as a functional parameter of gut maturation in neonates. These studies consistently show higher intestinal permeability in formula fed babies when compared to breast fed babies at 7-14 days postnatally, both in premature and term infants ${ }^{19,24,25}$. Taylor et al. found significantly higher intestinal permeability in formula fed preterm babies ( $\leq 32$ weeks) at postnatal day 7 and 14 , but not at postnatal day $30{ }^{19}$. Catassi et al. reported similar results in term neonates, with higher permeability at day 7 of life but similar gastrointestinal permeability 30 days after birth ${ }^{24}$. These data suggest differences in the time course of functional gut maturity for formula fed compared with breast fed babies. The current finding of delayed gut maturation in formula fed babies on postnatal day 12 , but not at postnatal day 19 are in line with the above mentioned studies.

Few markers are available for evaluating gut maturation and/or trophicity. This study indicates that I-FABP may provide such a tool, next to being an accurate marker of mesenteric ischemia, villous atrophy in coeliac disease and NEC 26-28. Citrulline has been proposed as a marker of gut maturity before, however urinary citrulline in preterm infants did not correlate with gut maturity in a recent study 29. As accurate measurement of gut maturity may be of clinical importance, for example in predicting feeding tolerance, further study of I-FABP as a marker of gut maturity is desirable.

Delayed intestinal maturation associated with increased intestinal permeability can play a role in the increased incidence of NEC in formula fed premature infants. Immaturity of the intestinal barrier can lead to easy passage of bacteria, viral particles and toxins across the mucosal membrane with excessive inflammation as a result. In line with this hypothesis, classical NEC typically presents at 10-12 days after birth ${ }^{30}$, the period in which intestinal maturation is still significantly lower in formula fed infants compared to breast fed infants. Moreover, these findings may give an explanation for breastfeeding as protective strategy in the treatment of NEC. The design of the study and the manner samples were collected did not enable us to analyze factors associated with impaired gut maturity, for example growth factors, of which most are quickly metabolized or not excreted in urine. Several growth factors, including insulin-like growth factor (IGF) ${ }^{31}$, epidermal 
growth factor (EGF) ${ }^{32}$, and transforming growth factor (TGF)- $\beta 2{ }^{33}$ have been linked to gut development. Moreover, colostrum induces higher enterocyte proliferation compared to mature milk ${ }^{34}$, and EGF levels are higher in preterm milk compared to term milk, as the preterm infant's need for trophic factors is high due to the lack of exposure to amniotic fluid ${ }^{35}$. Future studies investigating the role of human milk related growth factors can use urinary I-FABP as a read-out for gut maturation, which is easily obtainable in a non-invasive manner.

Alterations of the gut microbiome by breast milk may be another explanation for the observed difference between formula and breast milk, as early-life microbial colonization is crucial for maturation of the immune system ${ }^{36,37}$. Several studies have considered specific human milk oligosaccharides to be the most efficient breast milk substitutes in modulating microbial composition and NEC prevention due to their prebiotic function, particularly on beneficial Bifidobacterium species 37-39.

Another leading hypothesis for the beneficial effects of breast milk is its immunomodulatory and anti-inflammatory effect, largely orchestrated by concentrations of slgA, CD14, TGF- $\beta$, erythropoietin, and IL-10 ${ }^{40,41}$. It is however unlikely that inflammatory differences explain the differences in enterocyte maturation, as fecal calprotectin levels shortly after first enteral feeding were comparable between breast fed babies and formula fed babies, excluding the possibility of a low-grade inflammatory state in formula fed infants.

The current study has some limitations. Due to discharge to the referring hospitals or medium care wards, several patients were lost to follow up at day 19. On day 26 , more than $50 \%$ of patients had even dropped out. Therefore, representative statistical analysis on day 26 was not possible. On day 19, I-FABP values were almost twice as high in formula fed infants, and lack of statistical significance might have been caused by a lack of power at this time-point. A hypertrophic effect of formula feeding on the gut in this phase may cause such a difference ${ }^{42}$.

Further research should aim at unraveling the mechanisms by which breastfeeding improves intestinal maturation to design novel human milk substitutes, for example (analogues of) growth factors or human milk oligosaccharides. The preterm infant's high need for trophic factors should be taken into account. Furthermore, this study underlines the importance of breast milk use in preterm infants. Although evidence is limited, donor breast milk seems 
Chapter 9

to have the same beneficial effects as maternal breast milk when compared to formula feeding ${ }^{7}$. Therefore, efforts should be taken to stimulate human milk banking and routine use in preterm infants. 


\section{References}

1. Neu J, Walker WA. Necrotizing enterocolitis. N Engl J Med 2011;364:255-64.

2. Nanthakumar N, Meng D, Goldstein AM, et al. The mechanism of excessive intestinal inflammation in necrotizing enterocolitis: an immature innate immune response. PLoS One 2011;6:e17776.

3. Caplan MS, Simon D, Jilling T. The role of PAF, TLR, and the inflammatory response in neonatal necrotizing enterocolitis. Semin Pediatr Surg 2005;14:145-51.

4. Sullivan S, Schanler RJ, Kim JH, et al. An exclusively human milk-based diet is associated with a lower rate of necrotizing enterocolitis than a diet of human milk and bovine milk-based products. J Pediatr 2010;156:562-7 e1.

5. Boyd CA, Quigley MA, Brocklehurst P. Donor breast milk versus infant formula for preterm infants: systematic review and meta-analysis. Arch Dis Child Fetal Neonatal Ed 2007;92:F169-75.

6. McGuire W, Anthony MY. Donor human milk versus formula for preventing necrotising enterocolitis in preterm infants: systematic review. Arch Dis Child Fetal Neonatal Ed 2003;88:F11-4.

7. Quigley MA, Henderson G, Anthony MY, et al. Formula milk versus donor breast milk for feeding preterm or low birth weight infants. Cochrane Database Syst Rev 2007:CD002971.

8. Lucas A, Cole TJ. Breast milk and neonatal necrotising enterocolitis. Lancet 1990;336:1519-23.

9. Sangild PT, Siggers RH, Schmidt M, et al. Diet- and colonization-dependent intestinal dysfunction predisposes to necrotizing enterocolitis in preterm pigs. Gastroenterology 2006;130:1776-92.

10. Lieberman JM, Sacchettini J, Marks C, et al. Human intestinal fatty acid binding protein: report of an assay with studies in normal volunteers and intestinal ischemia. Surgery 1997;121:335-42.

11. Watanabe $\mathrm{K}$, Hoshi N, Tsuura $\mathrm{Y}$, et al. Immunohistochemical distribution of intestinal 15 kDa protein in human tissues. Arch Histol Cytol 1995;58:303-6.

12. Bullen TF, Forrest $S$, Campbell F, et al. Characterization of epithelial cell shedding from human small intestine. Lab Invest 2006;86:1052-63.

13. Derikx JP, Blijlevens NM, Donnelly JP, et al. Loss of enterocyte mass is accompanied by diminished turnover of enterocytes after myeloablative therapy in haematopoietic stem-cell transplant recipients. Ann Oncol 2009;20:337-42.

14. Reisinger KWE, M; Derikx, J.P.; Nikkels, P.G.; de Vries, B.; Adriaanse, M.P.; Jellema, R.K.; Kramer, B.W.; Wolfs, T.G. Intestinal fatty acid binding protein (I-FABP): a possible marker for gut maturation. Pediatr Res 2014; In press.

15. Cummins AG, Thompson FM. Effect of breast milk and weaning on epithelial growth of the small intestine in humans. Gut 2002;51:748-54.

16. Reisinger KW, Derikx JP, Thuijls G, et al. Noninvasive measurement of intestinal epithelial damage at time of refeeding can predict clinical outcome after necrotizing enterocolitis. Pediatr Res 2013;73:209-13. 
17. Fagerhol MK. Calprotectin, a faecal marker of organic gastrointestinal abnormality. Lancet 2000;356:1783-4.

18. van der Sluijs Veer G, van den Hoven B, Russel MG, et al. Time-resolved fluorimetric immunoassay of calprotectin: technical and clinical aspects in diagnosis of inflammatory bowel diseases. Clin Chem Lab Med 2006;44:292-8.

19. Taylor SN, Basile LA, Ebeling M, et al. Intestinal permeability in preterm infants by feeding type: mother's milk versus formula. Breastfeed Med 2009;4:11-5.

20. Derikx JP, Bijker EM, Vos GD, et al. Gut mucosal cell damage in meningococcal sepsis in children: relation with clinical outcome. Crit Care Med 2010;38:133-7.

21. Derikx JP, Vreugdenhil AC, Van den Neucker AM, et al. A pilot study on the noninvasive evaluation of intestinal damage in celiac disease using I-FABP and L-FABP. J Clin Gastroenterol 2009;43:727-33.

22. Thuijls G, Derikx JP, de Kruijf M, et al. Preventing enterocyte damage by maintenance of mean arterial pressure during major nonabdominal surgery in children. Shock 2012;37:22-7.

23. Derikx JP, Poeze M, van Bijnen AA, et al. Evidence for intestinal and liver epithelial cell injury in the early phase of sepsis. Shock 2007;28:544-8.

24. Catassi C, Bonucci A, Coppa GV, et al. Intestinal permeability changes during the first month: effect of natural versus artificial feeding. J Pediatr Gastroenterol Nutr 1995;21:383-6.

25. Weaver LT, Laker MF, Nelson R, et al. Milk feeding and changes in intestinal permeability and morphology in the newborn. J Pediatr Gastroenterol Nutr 1987;6:351-8.

26. Thuijls G, van Wijck K, Grootjans J, et al. Early diagnosis of intestinal ischemia using urinary and plasma fatty acid binding proteins. Ann Surg 2011;253:303-8.

27. Adriaanse MP, Tack GJ, Passos VL, et al. Serum I-FABP as marker for enterocyte damage in coeliac disease and its relation to villous atrophy and circulating autoantibodies. Aliment Pharmacol Ther 2013;37:482-90.

28. Ng EW, Poon TC, Lam HS, et al. Gut-Associated Biomarkers L-FABP, I-FABP, and TFF3 and LIT Score for Diagnosis of Surgical Necrotizing Enterocolitis in Preterm Infants. Ann Surg 2013;258:1111-8.

29. Bourdon A, Rouge C, Legrand A, et al. Urinary citrulline in very low birth weight preterm infants receiving intravenous nutrition. Br J Nutr 2012;108:1150-4.

30. Lin PW, Stoll BJ. Necrotising enterocolitis. Lancet 2006;368:1271-83.

31. Michiels J, De Vos M, Missotten J, et al. Maturation of digestive function is retarded and plasma antioxidant capacity lowered in fully weaned low birth weight piglets. $\mathrm{Br} \mathrm{J}$ Nutr 2013;109:65-75.

32. Troyer KL, Luetteke NC, Saxon ML, et al. Growth retardation, duodenal lesions, and aberrant ileum architecture in triple null mice lacking EGF, amphiregulin, and TGFalpha. Gastroenterology 2001;121:68-78.

33. Rautava S, Lu L, Nanthakumar NN, et al. TGF-beta2 induces maturation of immature human intestinal epithelial cells and inhibits inflammatory cytokine responses induced via the NF-kappaB pathway. J Pediatr Gastroenterol Nutr 2012;54:630-8. 
34. Tapper D, Klagsbrun M, Neumann J. The identification and clinical implications of human breast milk mitogen. J Pediatr Surg 1979;14:803-8.

35. Dvorak B, Fituch CC, Williams CS, et al. Increased epidermal growth factor levels in human milk of mothers with extremely premature infants. Pediatr Res 2003;54:15-9.

36. Maga EA, Weimer BC, Murray JD. Dissecting the role of milk components on gut microbiota composition. Gut Microbes 2013;4:136-9.

37. Oozeer R, van Limpt K, Ludwig $\mathrm{T}$, et al. Intestinal microbiology in early life: specific prebiotics can have similar functionalities as human-milk oligosaccharides. Am J Clin Nutr 2013;98:561S-71S.

38. Jantscher-Krenn E, Zherebtsov M, Nissan C, et al. The human milk oligosaccharide disialyllacto-N-tetraose prevents necrotising enterocolitis in neonatal rats. Gut 2012;61:1417-25.

39. Walker A. Milk and two oligosaccharides. Nat Rev Microbiol 2009;7:483.

40. Walker A. Breast milk as the gold standard for protective nutrients. J Pediatr 2010;156:S3-7.

41. Penttila IA, Flesch IE, McCue AL, et al. Maternal milk regulation of cell infiltration and interleukin 18 in the intestine of suckling rat pups. Gut 2003;52:1579-86.

42. Le Huerou-Luron I, Blat S, Boudry G. Breast- v. formula-feeding: impacts on the digestive tract and immediate and long-term health effects. Nutr Res Rev 2010;23:23-36. 



\section{Part 4 Biomarkers of necrotizing enterocolitis (NEC)}

The fourth and final aim was to assess the usefulness of gut-specific biomarkers in the detection, treatment and follow-up of pediatric surgery for NEC. In the previous part, the pathophysiology of gut maturation in the context of NEC was described. This provides increased knowledge to develop new preventive strategies. However, when NEC develops, early recognition and adequate treatment are indispensable. Chapter 10 describes a human study investigating non-invasive markers of intestinal cell damage and intestinal inflammation to detect NEC at an early time point, in babies who are clinically suspected of having NEC. In chapter 11, it was investigated whether non-invasive markers of intestinal cell damage and generalized inflammation can be used to select neonates with severe NEC, who require surgical intervention. In chapter 12, it was studied whether a marker of intestinal cell damage could be used to guide the clinical decision of restarting enteral feeds in patients treated for NEC. 

Chapter 10. Non-invasive measurement of fecal calprotectin and serum amyloid A (SAA) combined with intestinal Fatty Acid Binding Protein (I-FABP) in necrotizing enterocolitis (NEC)

Kostan W. Reisinger, Joep P.M. Derikx, David C. Van der Zee, Hens A.A. Brouwers, Boris W. Kramer, L.W. Ernest van Heurn, Wim A. Buurman. Journal of Pediatric Surgery 2012. 47(9):1640-5 


\begin{abstract}
Background

Diagnosis of necrotizing enterocolitis (NEC), an inflammatory intestinal disease mainly prevalent in prematures, remains challenging. Enterocyte damage in NEC can be assessed by intestinal Fatty Acid Binding Protein (I-FABP) with sensitivity of $93 \%$ and specificity of $90 \%$. Numerous markers of inflammation are known, such as serum amyloid $\mathrm{A}$ (SAA) and fecal calprotectin.
\end{abstract}

\title{
Purpose
}

To evaluate which combination of non-invasive measurement of inflammatory markers and I-FABP improves diagnostic accuracy in neonates suspected for NEC.

\section{Methods}

In 62 neonates with clinical suspicion of NEC (29 with final diagnosis of NEC), urinary I-FABP, urinary SAA, and fecal calprotectin levels were determined quantitatively. Diagnostic accuracy was calculated for the combinations I-FABPSAA and I-FABP-fecal calprotectin, using a multivariable logistic regression model.

\section{Results}

Combination of SAA and I-FABP did not increase diagnostic accuracy of I-FABP. However, combination of fecal calprotectin and I-FABP improved accuracy significantly. The combination of urinary I-FABP and fecal calprotectin measurement produced sensitivity $94 \%$, specificity $79 \%$, positive likelihood ratio 4.48 and negative likelihood ratio 0.08 .

\section{Conclusion}

The combination of non-invasive measurement of I-FABP and fecal calprotectin seems a promising way to diagnose NEC at an early time-point. Prospective analysis is required to confirm this finding and to evaluate better treatment strategies based on non-invasive measurement of I-FABP and calprotectin. 


\section{Introduction}

Necrotizing enterocolitis (NEC) is a severe neonatal gastrointestinal disease with high morbidity and mortality $(20-40 \%)$, characterized by inflammation and intestinal cell damage ${ }^{1}$. Diagnosis of NEC remains challenging, as the initial presentation is non-specific and often hard to distinguish from other gastrointestinal disorders and sepsis. While most laboratory tests and imaging techniques lack diagnostic accuracy ${ }^{2}$, measurement of intestinal Fatty Acid Binding Protein (I-FABP), a marker of intestinal cell damage, has been reported to discriminate NEC from other disease with high sensitivity and specificity ${ }^{3 \cdot 5}$. To determine the proper treatment strategy, it is of great importance to differentiate NEC at an early time point. Non-invasive measurement of diagnostic markers is preferred, to avoid the risk of anemia caused by blood sampling. This study evaluated whether non-invasive measurement of the inflammatory markers serum amyloid A (SAA) and fecal calprotectin could improve diagnostic accuracy of I-FABP in neonates with suspected NEC.

SAA is an acute-phase protein ( $11.5 \mathrm{kDa})$, synthesized by the liver upon induction by proinflammatory cytokines ${ }^{6}$. SAA was recently reported by $\mathrm{Ng}$ and colleagues to be a promising serum biomarker of both NEC and sepsis in neonates ${ }^{7}$. However, differentiation of NEC from other diseases is important, as several aspects of the treatment are different, including surgery in cases of persistent NEC ${ }^{8}$.

Calprotectin, a heterodimeric peptide (36 kDa), is released from the cytosol of neutrophils upon activation. Fecal calprotectin is a specific marker for neutrophil infiltrate in bowel mucosa. In intestinal inflammation, calprotectin is readily detectable in feces and plasma, making fecal calprotectin a suitable marker for NEC ${ }^{4,9,10}$.

We studied whether non-invasive measurement of the inflammatory markers SAA (in urine) and calprotectin (in feces) improves diagnostic accuracy of urinary I-FABP in neonates with gastrointestinal symptoms suspected of NEC.

\section{Patients and methods}

\section{Patients and sample collection}

62 consecutive patients with clinical suspicion of NEC were included in the neonatal intensive care units (NICU) at Maastricht University Medical Center and Wilhelmina Children's Hospital in Utrecht, between July 2005 and August 2010. Data on 34 (13 NEC) neonates have been published before ${ }^{4}$. Suspected 
NEC was defined as presence of abdominal distension, causing sufficient clinical concern to require abdominal X-ray, and/or to stop enteral feeding. Final diagnosis of NEC was made with the current golden standard of abdominal X-ray showing pneumatosis intestinalis (Bell stage II or higher) ${ }^{11}$. Written informed consent was obtained from both parents, and the study was conducted with approval from local medical ethical committees, and according to the revised version of the Declaration of Helsinki (October 2008, Seoul). The principles of good clinical practice (GCP) were followed during this study.

Urine from all included neonates was obtained at time of clinical suspicion of NEC by placing a dental cotton roll (Henry Schein, Almere, the Netherlands) in the diaper of the neonate. The rolls containing urine, were placed in a sterile $5 \mathrm{~mL}$ syringe (Becton Dickinson, Oxford, United Kingdom) and the urine was pressed into Micronic tubes (Micronic B.V., Lelystad, the Netherlands). Urine was stored at $-20^{\circ} \mathrm{C}$ until batch analysis. Stool samples were obtained and stored immediately at $-20^{\circ} \mathrm{C}$ until batch analysis. All laboratory analyses were performed by one person after completion of patient inclusion.

\section{Power analysis}

The number of patients needed for this study was calculated using the difference in SAA levels between controls and neonates with NEC/sepsis ${ }^{7}(\alpha=0.05,1-\beta=$ 0.95). This produced a minimum number of 9 patients per group.

\section{Urinary I-FABP measurement}

Urinary I-FABP was measured using an in-house enzyme-linked immunosorbent assay (ELISA) that selectively detects human I-FABP (lower detection limit: 12.5 $\mathrm{pg} / \mathrm{ml})$. Values are expressed as ratio ( $\mathrm{pg} / \mathrm{nmol}$ creatinine) of I-FABP $(\mathrm{pg} / \mathrm{ml})$ to creatinine $(\mathrm{nmol} / \mathrm{ml})$, to compensate for variations in urine concentration (I-FABP:Cr).

\section{Urinary SAA measurement}

Urinary SAA was measured using a commercially available ELISA kit (lower detection limit $15.0 \mathrm{ng} / \mathrm{ml}$ ), kindly provided by Hycult Biotechnology (Uden, the Netherlands). Values are expressed as ratio (pg/nmol creatinine) of SAA (ng/ml) to creatinine $(\mathrm{nmol} / \mathrm{ml})$ * 1000 , to compensate for variations in urine concentration (SAA:Cr). 


\section{Fecal calprotectin measurement}

After thawing of feces, $100 \mathrm{mg}$ was weighed and $4.9 \mathrm{ml}$ extraction buffer (0.1M Tris, $0.15 \mathrm{M} \mathrm{NaCl}, 1.0 \mathrm{M}$ urea, $10 \mathrm{mM} \mathrm{CaCl}_{2} \cdot 2 \mathrm{H}_{2} \mathrm{O}, 0.1 \mathrm{M}$ citric acid, $0.5 \% \mathrm{BSA}, \mathrm{pH}$ 8.0) was added ${ }^{12}$. After 30 minutes shaking, $1 \mathrm{ml}$ of suspension was centrifuged at $10,000 \mathrm{rpm}$ for 20 minutes at $4^{\circ} \mathrm{C}$ and supernatant was aliquoted and stored at $-20^{\circ} \mathrm{C}$. Calprotectin concentration was measured in lysate using the commercially available calprotectin ELISA (lower detection limit $625 \mathrm{ng} / \mathrm{ml}$ ), kindly provided by Hycult Biotechnology (Uden, the Netherlands). Fecal calprotectin concentration is given in $\mu \mathrm{g}$ calprotectin per gram feces.

\section{Statistical analyses}

Normality was tested by Kolmogorov-Smirnov. Mann-Whitney $U$ test was used for between group comparison for continuous data. Dichotomous variables were compared using Fisher exact test. All data are presented as median and range.

To determine the accuracy of I-FABP combined with an inflammatory marker in the diagnosis of NEC, two combinations of markers were tested: I-FABP-SAA and I-FABP-calprotectin. Predicted probabilities for the presence of NEC were calculated for both combinations using logistic regression analysis and then plotted in receiver operator characteristic (ROC) curves. Overall diagnostic accuracy of I-FABP-SAA and I-FABP-calprotectin was represented by the area under the curve (AUC). The best cut-off point of predicted probabilities $(P)$ was defined as the cut-off point with maximum sum of sensitivity and specificity. To calculate the linear function describing all combinations of ideal cut-off values for I-FABP-SAA and I-FABP-calprotectin in the diagnosis of NEC, the cut-off point $(P)$ was used in the following equation: $\operatorname{Ln}(P /(1-P))=B_{0}+B_{1} X_{1}+B_{2} X_{2}$; in which $B_{0}$ represents the constant of the logistic regression analysis and $B_{1}$ and $B_{2}$ represent the logistic regression coefficients of I-FABP and SAA or I-FABP and calprotectin, respectively. By calculating coordinates of intersections with the $x$ - and $y$-axis, the linear functions describing the cut-off lines of I-FABP-SAA and I-FABP-calprotectin could be determined.

Statistical analyses were performed with Prism 5.0 for Windows (GraphPad Software Inc. San Diego, CA) and SPSS 15.0 for Windows (SPSS Inc. Chicago, IL). STARD statement for reporting studies of diagnostic accuracy was used in this study ${ }^{13}$. 


\section{Results}

\section{Patients}

62 neonates were included, of whom 32 were male. Median gestational age was 215 [175 - 289] days, median birth weight was 1328 [585 - 3570] g. 29 infants were diagnosed with NEC (47\%).

There were no significant differences in gestational age, birth weight, or sex between the groups (Table 1). Final diagnoses of prematures without NEC are listed in Table 2.

TABLE 1. Baseline characteristics

\begin{tabular}{lllll}
\hline & NEC $(n=29)$ & No NEC $(n=33)$ & Total $(n=62)$ & $P$ \\
\hline Gestational age & $215(184-268)$ & $213(175-289)$ & $215(175-289)$ & 0.72 \\
Birth weight & $1400(860-1960)$ & $1270(585-3570)$ & $1328(585-3570)$ & 0.67 \\
Sex & $11 \mathrm{M}(38 \%)$ & $21 \mathrm{M}(64 \%)$ & $32 \mathrm{M}(52 \%)$ & 0.07 \\
\hline
\end{tabular}

Data are presented as median (range)

TABLE 2. Diagnoses associated with abdominal symptoms in no NEC group

\begin{tabular}{ll}
\hline CoNS sepsis & 17
\end{tabular}

Gastrointestinal symptoms of unknown origin 10

Norovirus 2

Portal vein thrombosis $\quad 2$

Rotavirus $\quad 1$

Enterococcus faecalis sepsis $\quad 1$

Enterobacter cloacae sepsis 1

Constipation 1

Hypomotility 1

M. Hirschprung 1

Gastroschisis 1

Total 38

* 5 patients had 2 diagnoses associated with abdominal symptoms

CoNS = coagulase negative staphylococcus 


\section{Single marker analyses}

\section{I-FABP:Cr}

Median I-FABP:Cr levels in neonates with NEC were significantly higher compared to infants with other diseases (respectively, 11.4 [0.4 - 4,878] pg/nmol, and 1.2 [0.0 - 11.0] pg/nmol, $p<0.001$ ). An ideal cut-off value of $2.4 \mathrm{pg} / \mathrm{nmol}$ was found with sensitivity $79 \%$, specificity $85 \%$, positive likelihood ratio (LR+) 5.23 , and negative likelihood ratio (LR-) 0.24 (Figure 1). The ROC curve yielded an area under the curve (AUC) of 0.88 (95\% confidence interval (CI) $0.80-0.97$ ).

\section{SAA:Cr}

Median SAA:Cr levels in neonates with NEC were significantly higher compared to infants with other diagnoses (respectively, 44.4 [9.3 - 31,127] pg/nmol, and 21.5 [12.0 - 1,469], $p=0.009)$. The AUC of the ROC curve was 0.70 (95\% Cl 0.56 - 0.83), with an ideal cut-off value of $42.2 \mathrm{pg} / \mathrm{nmol}$ (sensitivity $52 \%$, specificity 91\%, LR+ 5.69, LR- 0.53).

\section{Calprotectin}

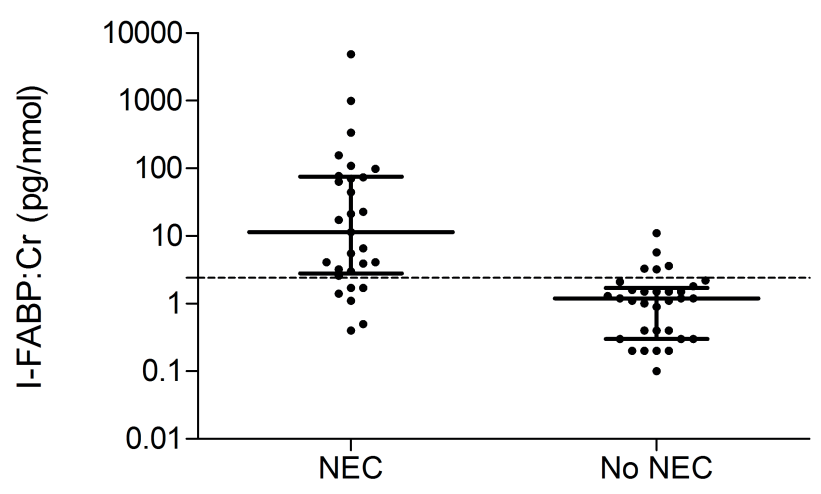

FIGURE 1. Urinary I-FABP levels when NEC is clinically suspected can accurately distinguish between neonates with final diagnosis of NEC and those with other final diagnoses. A cut-off value of $2.4 \mathrm{pg} /$ $\mathrm{nmol}$ creatinine is depicted by the dotted line. 


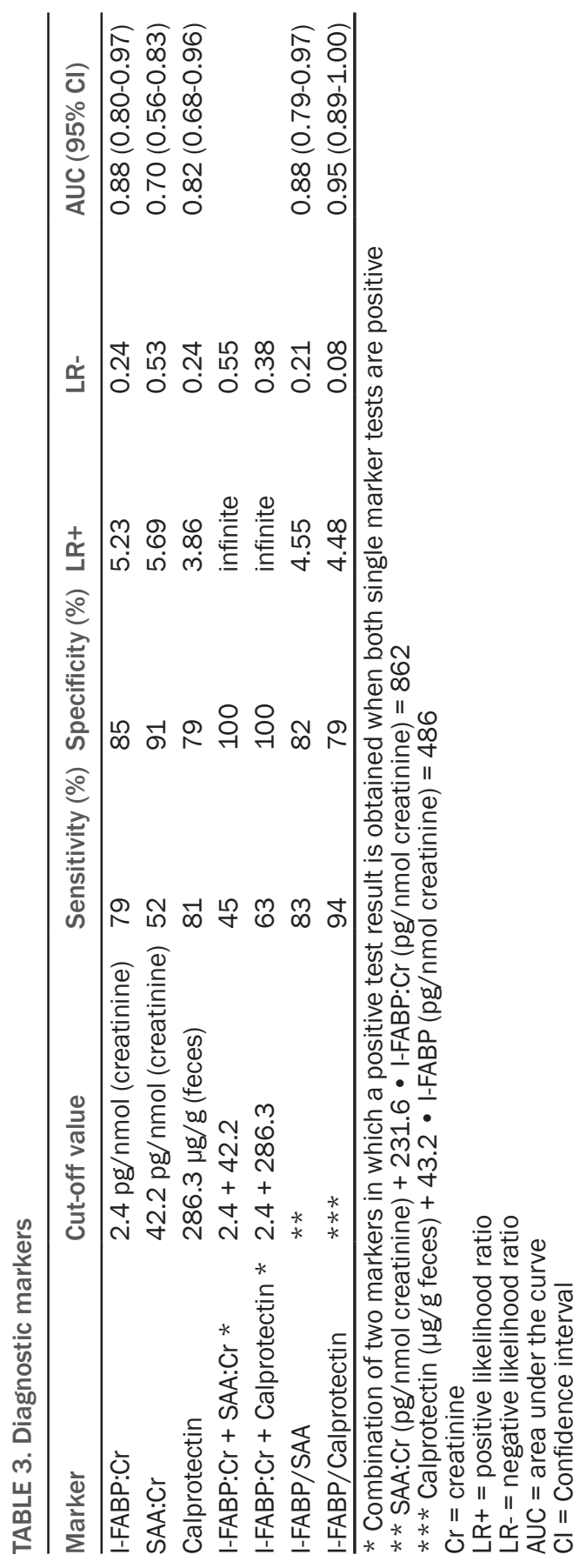


Stool samples were only available in 35 out of 62 neonates (16 with NEC (55\%) and 19 with other final diagnoses (58\%)). Median calprotectin levels in neonates with NEC were significantly higher compared to infants with other diagnoses (respectively, 402.2 [107.6 - 847.6] $\mu \mathrm{g} / \mathrm{g}$ feces, and 79.6 [1.0 - 625.1], $\mathrm{p}=0.001$ ). The AUC of the ROC curve was $0.82(95 \% \mathrm{Cl} 0.68-0.96)$, with an ideal cut-off value of $286.3 \mu \mathrm{g} / \mathrm{g}$ feces (sensitivity $81 \%$, specificity $79 \%$, LR+ 3.86, LR- 0.24).

\section{Multiple marker analyses}

First, the combinations of I-FABP with SAA and I-FABP with fecal calprotectin were analyzed by combining the cut-off values found in the single marker analyses. This implies that a positive test result is obtained when both values are above the cut-off value, i.e. I-FABP $>2.4 \mathrm{pg} / \mathrm{nmol}$ and SAA $>42.2 \mathrm{pg} / \mathrm{nmol}$. Combination of I-FABP:CR and SAA:Cr yielded sensitivity $45 \%$, specificity $100 \%$, LR+ infinite, and LR- 0.55 (Table 3). Combination of I-FABP:Cr and fecal calprotectin produced sensitivity 63\%, specificity 100\%, LR+ infinite, and LR- 0.38 (Table 3 ). In this analysis, no ROC curve can be calculated.

Second, a logistic regression analysis approach was used to analyze the combinations of I-FABP with SAA and I-FABP with fecal calprotectin.

\section{I-FABP:Cr and SAA:Cr}

When I-FABP:Cr and SAA:Cr were combined, a cut-off line was calculated with sensitivity $83 \%$, specificity $82 \%$, LR+ 4.55 , and LR- 0.21 (Figure 2), thereby not increasing diagnostic accuracy compared to measurement of I-FABP:Cr. The cutoff line was described by the linear function: $[\mathrm{SAA} / \mathrm{Cr}(\mathrm{pg} / \mathrm{nmol}$ creatinine $)]+$ 231.6 - $[\mathrm{I}-\mathrm{FABP} / \mathrm{Cr}(\mathrm{pg} / \mathrm{nmol}$ creatinine $)]=862$; which means that a positive test is obtained when any combination of SAA:Cr and I-FABP:Cr levels in this formula results in a value $>862$. AUC of the ROC curve for discrimination of neonates with NEC from those with other final diagnoses was $0.88(95 \% \mathrm{Cl} 0.79-0.97)$.

\section{I-FABP:Cr and fecal calprotectin}

When I-FABP:Cr was combined with fecal calprotectin in differentiating NEC from other final diagnoses, a cut-off line was calculated with sensitivity $94 \%$, specificity $79 \%, \mathrm{LR}+4.48$, and LR- 0.08 (Figure 3). The cut-off line was described by the linear function: [Calprotectin $(\mathrm{\mu g} / \mathrm{g}$ feces $)]+43.2 \cdot[\mathrm{I}-\mathrm{FABP} / \mathrm{Cr}(\mathrm{pg} / \mathrm{nmol}$ creatinine $)]$ 
= 486; which means that a positive test is obtained when any combination of calprotectin and I-FABP levels in this formula results in a value $>486$. AUC of the ROC curve was 0.95 (95\% Cl $0.89-1.00)$.

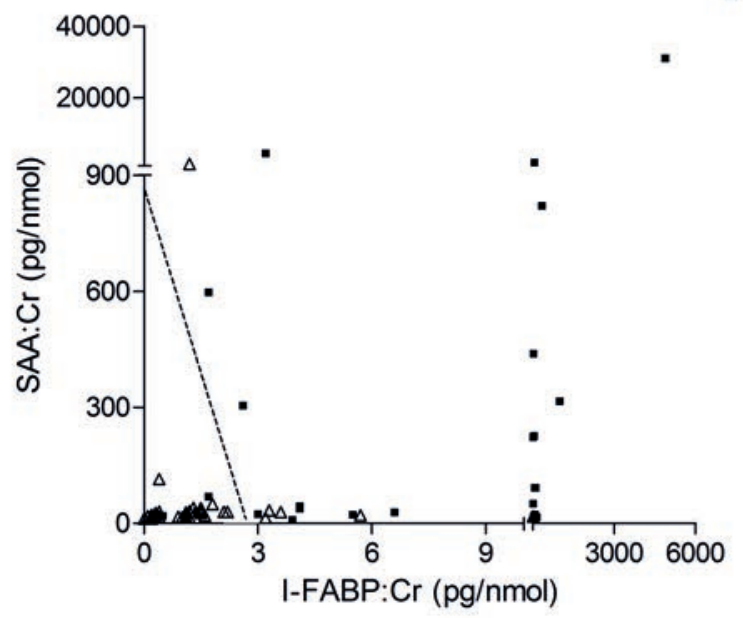

FIGURE 2. Combination of urinary I-FABP levels and urinary SAA levels in neonates with final diagnosis of NEC (squares) and those with other final diagnoses (triangles) when NEC is clinically suspected. The ideal cut-off line for differentiating between NEC and other diagnoses is depicted by the dotted line.

Since the combination of I-FABP:Cr and fecal calprotectin yielded the highest diagnostic accuracy, multivariable logistic regression model was applied to evaluate whether this combination of markers also could differentiate Bell stage III from Bell stage II at the moment of clinical suspicion of NEC. This resulted in an AUC of the ROC curve of 0.55 (95\% Cl $0.24-0.86), p=0.75$. These data show that measurement of fecal calprotectin increases diagnostic accuracy of urinary I-FABP, however it is not useful to differentiate Bell stage III from Bell stage II.

\section{Discussion}

This study aimed to assess whether non-invasive measurement of the inflammatory markers SAA (in urine) and calprotectin (in feces) improves diagnostic accuracy of the enterocyte damage marker I-FABP in neonates with gastrointestinal symptoms 


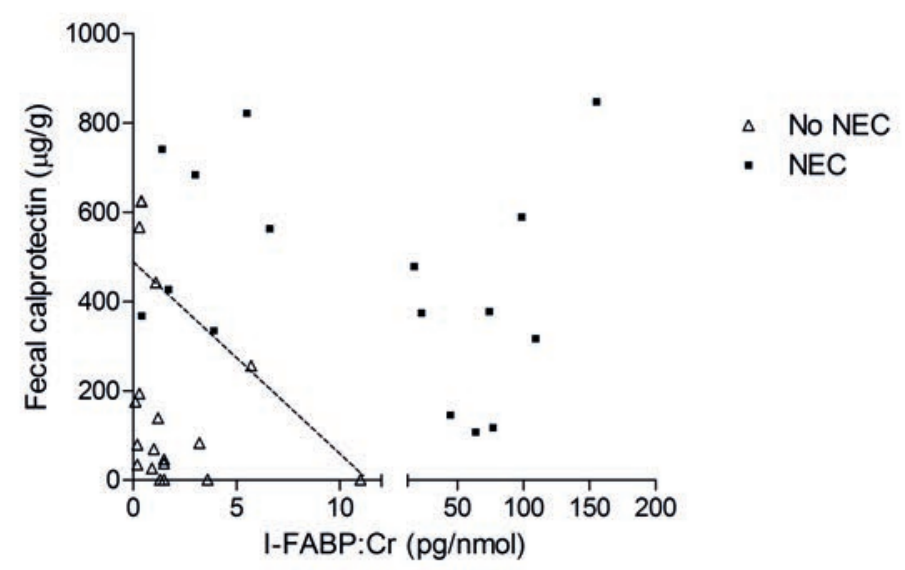

FIGURE 3. Combination of urinary I-FABP levels and fecal calprotectin levels in neonates with final diagnosis of NEC (squares) and those with other final diagnoses (triangles) when NEC is clinically suspected. The ideal cut-off line for differentiating between NEC and other diagnoses is depicted by the dotted line. Note: stool samples were only available in 35 out of 62 neonates.

suspected of NEC. The data show that measurement of fecal calprotectin, but not SAA improves diagnostic accuracy of urinary I-FABP measurement in diagnosing NEC.

In the current study, only non-invasive markers were investigated, as blood sampling in neonates should be avoided ${ }^{14}$, and because a clinical benefit of the markers has never been demonstrated. Classic parameters of infection (C-reactive protein, white blood cell count, platelets) are therefore not presented here. A limitation of non-invasive measurement of SAA is that little is known on the plasma clearance of SAA. Although urinary SAA levels were detected in a large part of the patients, further research is needed to elucidate the renal clearance of SAA.

Cetinkaya et al. showed that plasma SAA levels were elevated in neonates with NEC compared to neonates with sepsis at moment of diagnosis ${ }^{15}$. In contrast however, Eras et al. reported higher plasma levels of SAA in children with sepsis, compared to neonates with NEC ${ }^{16}$. In our cohort, measurement of urinary SAA alone was not useful as a diagnostic marker for NEC, due to the low sensitivity. In a recent study by $\mathrm{Ng}$ et al. ${ }^{7}$, SAA was reported to be a highly specific marker of both NEC and sepsis in low birth weight neonates. Importantly, the authors did not differentiate between NEC and sepsis. While initial medical treatment is 
similar for both NEC and sepsis, resection of affected bowel is essential in NEC when signs of perforation occur or when symptoms do not improve on medical treatment. Here, we aimed to distinguish NEC from other diagnoses including sepsis at an early time-point. Unfortunately, inclusion of urinary SAA levels did not substantially improve the diagnostic accuracy of urinary I-FABP measurement in diagnosing NEC. This might be due to a lack of specificity of SAA being a marker of generalized inflammation.

Next this study aimed to include intestinal inflammation and more specifically fecal calprotectin. It increased the diagnostic accuracy of I-FABP measurement. Elevated levels of fecal calprotectin in NEC are in line with several studies 4, 9, 17 . In a previous study of our institute however, fecal calprotectin did not improve diagnostic accuracy of I-FABP ${ }^{4}$. This could have been a consequence of the higher sensitivity and specificity of I-FABP than found in the current cohort.

The diagnostic accuracy improved when measurement of fecal calprotectin was combined with I-FABP. The data demonstrated a substantial increase in sensitivity and a decrease in negative likelihood ratio, which is important in possibly lethal diseases like NEC. When applying Bayes theorem ${ }^{18,19}$ with NEC prevalence $47 \%$ in the studied cohort, a negative test result of I-FABP alone produces a post-test probability of $18 \%$ (95\% Cl 10\%-31\%). This means that $18 \%$ of neonates with NEC are missed by the test (18\% false-negatives). When both I-FABP and fecal calprotectin are measured, the post-test probability of a negative test decreases to $7 \%$ (95\% Cl 2\%-22\%), which means that only $7 \%$ of neonates with NEC are missed by the test. The post-test probability when a positive test result is acquired is $82 \%$ (95\% Cl 67\%-91\%) for I-FABP and 80\% (95\% Cl 67\%-89\%) for I-FABP combined with fecal calprotectin, which is comparable. The benefit of the combination of I-FABP and calprotectin lies thus in a lower rate of missed NEC diagnoses.

The combination of two diagnostic markers allows to calculate a cut-off line instead of a single cut-off value, since we found that single markers or combination of static cut-off points were not clinically relevant enough to differentiate NEC from other final diagnoses in neonates suspected for NEC. This cut-off line could be described as [Calprotectin ( $\mu \mathrm{g} / \mathrm{g}$ feces)] + 43.2 • [I-FABP/Cr (pg/nmol creatinine)] $=486$, which means that a positive test is obtained when any combination of fecal calprotectin and urinary I-FABP levels in this formula results in a value $>486$. For clinical application, a table or graph should be used when quick decision making is required. Since feces was obtained only in 35 out of 62 neonates (56\%), the 
combined method was only possible in $56 \%$ of the children. Further prospective studies are needed to confirm these results in a larger population and to evaluate the effects of treatment strategies based on calprotectin and I-FABP measurement.

In conclusion, this study shows that non-invasive measurement of SAA adds little to the diagnostic accuracy of urinary I-FABP in diagnosing NEC while fecal calprotectin increases the diagnostic accuracy in children who produce feces. The combination of urinary I-FABP and fecal calprotectin seems a promising noninvasive way to diagnose NEC at an early time-point. 


\section{References}

1. Neu J, Walker WA. Necrotizing enterocolitis. N Engl J Med 2011;364:255-64.

2. Hallstrom M, Koivisto AM, Janas M, et al. Laboratory parameters predictive of developing necrotizing enterocolitis in infants born before 33 weeks of gestation. J Pediatr Surg 2006;41:792-8.

3. Guthmann F, Borchers T, Wolfrum C, et al. Plasma concentration of intestinal- and liver-FABP in neonates suffering from necrotizing enterocolitis and in healthy preterm neonates. Mol Cell Biochem 2002;239:227-34.

4. Thuijls G, Derikx JP, van Wijck K, et al. Non-invasive markers for early diagnosis and determination of the severity of necrotizing enterocolitis. Ann Surg 2010;251:1174-80.

5. Derikx JP, Evennett NJ, Degraeuwe PL, et al. Urine based detection of intestinal mucosal cell damage in neonates with suspected necrotising enterocolitis. Gut 2007;56:14735.

6. Malle E, Steinmetz A, Raynes JG. Serum amyloid A (SAA): an acute phase protein and apolipoprotein. Atherosclerosis 1993;102:131-46.

7. Ng PC, Ang IL, Chiu RW, et al. Host-response biomarkers for diagnosis of lateonset septicemia and necrotizing enterocolitis in preterm infants. J Clin Invest 2010;120:2989-3000.

8. Lin PW, Stoll BJ. Necrotising enterocolitis. Lancet 2006;368:1271-83.

9. Carroll D, Corfield A, Spicer R, et al. Faecal calprotectin concentrations and diagnosis of necrotising enterocolitis. Lancet 2003;361:310-1.

10. Fagerhol MK. Calprotectin, a faecal marker of organic gastrointestinal abnormality. Lancet 2000;356:1783-4.

11. Bell MJ, Ternberg JL, Feigin RD, et al. Neonatal necrotizing enterocolitis. Therapeutic decisions based upon clinical staging. Ann Surg 1978;187:1-7.

12. van der Sluijs Veer G, van den Hoven B, Russel MG, et al. Time-resolved fluorimetric immunoassay of calprotectin: technical and clinical aspects in diagnosis of inflammatory bowel diseases. Clin Chem Lab Med 2006;44:292-8.

13. Bossuyt PM, Reitsma JB, Bruns DE, et al. The STARD statement for reporting studies of diagnostic accuracy: explanation and elaboration. Clin Chem 2003;49:7-18.

14. Strauss RG. Neonatal anemia: pathophysiology and treatment. Immunol Invest 1995;24:341-51.

15. Cetinkaya M, Ozkan $\mathrm{H}$, Koksal $\mathrm{N}$, et al. The efficacy of serial serum amyloid $A$ measurements for diagnosis and follow-up of necrotizing enterocolitis in premature infants. Pediatr Surg Int 2010;26:835-41.

16. Eras Z, Oguz S, Dizdar EA, et al. Serum amyloid-A levels in neonatal necrotizing enterocolitis. J Clin Lab Anal 2011;25:233-7.

17. Campeotto F, Baldassarre M, Butel MJ, et al. Fecal calprotectin: cutoff values for identifying intestinal distress in preterm infants. J Pediatr Gastroenterol Nutr 2009;48:507-10.

18. Hall GH. The clinical application of Bayes' theorem. Lancet 1967;2:555-7.

19. Fagan TJ. Letter: Nomogram for Bayes theorem. N Engl J Med 1975;293:257. 


Chapter 11. Non-invasive serum amyloid A (SAA) measurement and plasma platelets for accurate prediction of surgical intervention in severe necrotizing enterocolitis (NEC)

Kostan W. Reisinger, Boris W. Kramer, David C. Van der Zee, Hens A.A. Brouwers, Wim A. Buurman, L.W. Ernest van Heurn, Joep P.M. Derikx. PLoS One 2014. 7(1):e30539 


\section{Abstract \\ Objective}

To evaluate the value of biomarkers to detect severe NEC.

\section{Summary background data}

The time point of surgery in necrotizing enterocolitis (NEC) is critical. Therefore, there is a need for markers that detect severe NEC, because clinical signs of severe NEC often develop late. This study evaluated the value of biomarkers reflecting intestinal cell damage and inflammation to detect severe NEC.

\section{Methods}

29 neonates with NEC were included. Two definitions of moderate versus severe NEC were analyzed: medical NEC $(n=12)$ versus surgical or fatal NEC $(n=17)$; and Bell stage II NEC $(n=13)$ versus stage III NEC $(n=16)$. Urinary intestinal fatty acid binding protein (I-FABP), serum amyloid A (SAA), C3a and C5a, and fecal calprotectin were measured. C-reactive protein (CRP), white blood cell count (WBC) and platelet count data were measured in blood.

\section{Results}

In both definitions of moderate versus severe NEC, urinary SAA levels were significantly higher in severe NEC. A cut-off value of $34.4 \mathrm{ng} / \mathrm{ml}$ was found in surgical NEC versus medical NEC (sensitivity, 83\%; specificity, 83\%; LR+, 4.88 (95\% Cl, 1.37 -17.0); LR-, 0.20 (95\% Cl, 0.07 - 0.60)) at diagnosis of NEC and at one day prior to surgery in neonates who were operated later on. Combination of urinary SAA and platelet count increased the accuracy, with a sensitivity, 94\%; specificity, 83\%; LR+, 5.53 (95\% Cl, 1.57 - 20.0); and LR-, 0.07 (95\% Cl, 0.01 0.48).

\section{Conclusion}

Urinary SAA is an accurate marker in differentiating severe NEC from moderate NEC; particularly if combined with serum platelet count. 


\section{Introduction}

Necrotizing enterocolitis (NEC) is the most severe gastrointestinal disorder in neonates, affecting predominantly premature infants ${ }^{1}$. Initial treatment consists of discontinuation of enteral feeding, intravenous administration of broad spectrum antibiotics, and cardiopulmonary support. In case of perforation or severe clinical deterioration on medical treatment, resection of affected bowel is often the treatment of choice ${ }^{2}$. This group carries the highest mortality ${ }^{3}$, and it is therefore essential to detect these severe cases at an early time point. In addition to clinical signs and symptoms, various plasma markers have been described to correlate with the severity of NEC, including serum amyloid A (SAA) ${ }^{4}$, intestinal Fatty Acid Binding Protein (I-FABP) ${ }^{5,6}{ }^{6}$, E-selectin ${ }^{7}, \mathrm{C}^{5} \mathrm{a}^{8}$ and IL-6 ${ }^{9}$. As venous puncture is a delicate and unfavorable procedure in neonates and a primary cause of anemia among preterm infants ${ }^{10,11}$, it is highly desirable to use non-invasive methods to discriminate neonates with severe NEC from those that can be treated conservatively. Non-invasive measurement for predicting NEC severity has only been described for I-FABP (14 kDa) ${ }^{12,13}$. Another protein which is a candidate for non-invasive measurement for its small molecular size is the plasma marker SAA. SAA is an acute-phase protein ( $11.5 \mathrm{kDa})$, quickly synthesized by the liver and the kidneys upon induction by pro-inflammatory cytokines ${ }^{14-16}$.

Calprotectin, a heterodimeric peptide, is released from the cytosol of neutrophils upon activation. Fecal calprotectin is a specific marker for neutrophil infiltrate in bowel mucosa. In intestinal inflammation, calprotectin is readily detectable in feces and plasma, making fecal calprotectin a suitable marker for NEC and possibly for NEC severity ${ }^{12,17,18}$.

Complement activation has been linked to the pathogenesis of NEC ${ }^{19}$; plasma levels of $\mathrm{C} 5 \mathrm{a}$ are associated with perforation and death in patients with NEC 8. The complement activation products $\mathrm{C} 3 \mathrm{a}$ and $\mathrm{C} 5 \mathrm{a}$ are important in all three complement pathways. Their small molecular size (both $11 \mathrm{kDa}$ ) renders these proteins suitable as urinary markers of NEC severity.

This study evaluated the accuracy of non-invasive urinary and fecal measurement of markers reflecting intestinal cell damage and inflammation in addition to classical serum markers of inflammation in predicting disease severity of NEC. Furthermore, this study provided control values of non-invasive markers in premature neonates. 


\section{Patients and methods}

\section{Ethics statement}

Written informed consent was obtained from both parents, and the study was approved by the medical ethical committee of Maastricht University Medical Centre, according to the revised version of the Declaration of Helsinki (October 2008, Seoul). The principles of good clinical practice (GCP) were followed during this study.

\section{Patients and sample collection}

All patients with NEC in the neonatal intensive care units (NICU) at Maastricht University Medical Centre and Wilhelmina Children's Hospital in Utrecht, between January 2008 and August 2010 were included into this study. In all patients, NEC was confirmed with the current golden standard of abdominal X-ray showing pneumatosis intestinalis (Bell stage II or higher, Table 1$)^{20}$. In this study, medical NEC was defined as Bell stage II or higher without the need for surgery and not resulting in death. The following definitions of moderate versus severe NEC were used; first, a clinical definition: medical NEC versus surgical or fatal NEC; and second, a theoretical definition: Bell stage II NEC versus Bell stage III NEC. The theoretical definition is widely used, however the clinical definition may be more appropriate to evaluate the need for surgery, which is a clinical decision. The need for surgery was determined by a pediatric surgeon together with the attending neonatologist.

Urine from all included neonates was collected at time of clinical diagnosis of NEC and daily until discharge from the NICU by placing a dental cotton roll (Henry Schein, Almere, the Netherlands) in the diaper of the neonate. Once the roll was filled with urine, it was placed in a sterile $5 \mathrm{~mL}$ syringe (Becton Dickinson, Oxford, United Kingdom), the urine was transferred into Micronic tubes (Micronic B.V., Lelystad, the Netherlands) and stored at $-20^{\circ} \mathrm{C}$ until batch analysis. In all neonates, urine samples at time of diagnosis (D) were analyzed for the current study. It is unclear whether measurement at this single time point provides optimal accuracy or whether the accuracy changes with ongoing disease. Therefore, urine samples collected at two days before surgery (S-2) and one day before surgery (S-1) were also analyzed in neonates operated on two or more days following diagnosis. This method enabled to better assess the potential predictive value of markers for surgery in this cohort. 
Stool samples were obtained at diagnosis and stored immediately at $-20^{\circ} \mathrm{C}$ until batch analysis. All analyses were performed by one person after completion of patient inclusion, who was unaware of clinical outcome.

To determine control values of these biomarkers, 20 consecutive premature infants (matched with NEC infants for birth weight and gestational age at birth) admitted to the NICU at Maastricht University Medical Centre, without gastrointestinal symptoms were included. In these premature neonates serving as controls, a urine sample and a feces sample was collected at one week following birth. All control neonates were on full enteral feeding by that time and had not experienced feeding problems.

\section{Urinary I-FABP measurement}

Urinary I-FABP was measured using an in-house enzyme-linked immunosorbent assay (ELISA) that selectively detects human I-FABP (lower detection limit: 12.5 $\mathrm{pg} / \mathrm{ml})$.

\section{Urinary SAA measurement}

Urinary SAA was measured using a commercially available ELISA kit (lower detection limit $15.0 \mathrm{ng} / \mathrm{ml}$ ), kindly provided by Hycult Biotechnology (Uden, the Netherlands).

\section{Urinary C3a and C5a measurement}

Urinary C3a and C5a were measured using commercially available ELISA kits (C3a, lower detection limit $250 \mathrm{pg} / \mathrm{ml}$; C5a, lower detection limit 1,250 pg/ml), kindly provided by Hycult Biotechnology (Uden, the Netherlands).

\section{Fecal calprotectin measurement}

After thawing of feces, $100 \mathrm{mg}$ was weighed and added to $4.9 \mathrm{ml}$ extraction buffer $\left(0.1 \mathrm{M}\right.$ Tris, $0.15 \mathrm{M} \mathrm{NaCl}, 1.0 \mathrm{M}$ urea, $10 \mathrm{mM} \mathrm{CaCl} \cdot 2 \mathrm{H}_{2} \mathrm{O}, 0.1 \mathrm{M}$ citric acid, $0.5 \%$ $\mathrm{BSA}, \mathrm{pH} \mathrm{8.0)}{ }^{21}$. After 30 minutes shaking, $1 \mathrm{ml}$ of suspension was centrifuged at $10,000 \mathrm{rpm}$ for 20 minutes at $4^{\circ} \mathrm{C}$ and supernatant was aliquoted and stored at $-20^{\circ} \mathrm{C}$. Calprotectin concentration was measured in lysate using the commercially 
available calprotectin ELISA (lower detection limit $625 \mathrm{ng} / \mathrm{ml}$ ), kindly provided by Hycult Biotechnology (Uden, the Netherlands). Fecal calprotectin concentration is given in $\mu$ g calprotectin per gram feces.

\section{Conventional blood laboratory tests}

Classical markers of inflammation (C-reactive protein (CRP), white blood cell count (WBC) and platelet count) were determined as part of routine care by the departments of clinical chemistry and were collected from the medical records.

\section{Statistical analyses}

Normality was tested by Kolmogorov-Smirnov. Mann-Whitney $U$ test was used for between group comparisons for continuous data. Pairwise comparisons were analyzed using Wilcoxon Signed Ranks test. Dichotomous variables were compared using Fisher exact test. All data are presented as median and range. Receiver operating characteristic (ROC) curves were used to calculate the accuracy of the studied markers predicting disease severity. The ideal cut-off value for diagnosing severe NEC was defined as the cut off value with maximum sum of sensitivity and specificity.

To determine the accuracy of combined markers detecting severe NEC, logistic regression analysis was performed and then plotted in receiver operator characteristic (ROC) curves. Overall diagnostic accuracy of combined markers was represented by the area under the curve (AUC). The best cut-off point of predicted probabilities $(P)$ was defined as the cut-off point with maximum sum of sensitivity and specificity. To calculate the linear function describing all combinations of ideal cut-off values for combined markers in the detection of severe of NEC, the cutoff point $(P)$ was used in the following equation: $\operatorname{Ln}(P /(1-P))=B_{0}+B_{1} X_{1}+B_{2} X_{2}$; in which $B_{0}$ represents the constant of the logistic regression analysis and $B_{1}$ and $B_{2}$ represent the logistic regression coefficients of SAA and platelet count, respectively. By calculating coordinates of intersections with the $x$ - and $y$-axis, the linear function describing the cut-off line of SAA and platelet count could be determined.

Sample size was calculated as follows: in a previous study, mean I-FABP levels were $36 \mathrm{pg} / \mathrm{nmol}$ creatinine in infants with severe NEC compared with $5 \mathrm{pg} / \mathrm{nmol}$ creatinine in infants with mild NEC ${ }^{12}$. With $\alpha=0.05$ and $1-\beta=0.80$, this resulted in a minimal sample size of 12 patients per group. 
Statistical analyses were performed with Prism 5.0 for Windows (GraphPad Software Inc. San Diego, CA) and SPSS 15.0 for Windows (SPSS Inc. Chicago, IL). STARD statement for reporting studies of diagnostic accuracy was used in this study ${ }^{22}$.

\section{Results}

\section{Patients}

Twenty-nine consecutive neonates (11 males) were included. Median gestational age was 30+5 [range: $26+2-38+2$ ] weeks, median birth weight was 1400 [860 - 1960] g. Two definitions of moderate versus severe NEC were used; medically treated NEC (medical NEC group $n=12$ ) versus NEC requiring operation or causing death (operative/fatal NEC group, $n=17$ ), and NEC stage II (NEC II group, $n=13$ ) versus NEC stage III (NEC III group, $n=16$ ), based on the modified Bell's staging criteria according to Walsh and Kliegman ${ }^{23}$ (Table 1).

There were no significant differences in birth weight and sex between the medical NEC and operative/fatal NEC groups (Table 2A), or between the NEC II and NEC III groups (Table 2B). Gestational age however was significantly lower in the operative/fatal NEC group compared with the medical NEC group and in the NEC III group compared with the NEC II group.

TABLE 1. Modified Bell's criteria according to Walsh and Kliegman

\begin{tabular}{|c|c|c|c|}
\hline Stage & Clinical findings & Radiographic findings & Gastrointestinal findings \\
\hline IA & $\begin{array}{l}\text { Apnea and bradycardia, } \\
\text { temperature instability }\end{array}$ & $\begin{array}{l}\text { Normal or intestinal } \\
\text { dilation, mild ileus }\end{array}$ & $\begin{array}{l}\text { Gastric residuals, emesis, mild } \\
\text { abdominal distention }\end{array}$ \\
\hline IB & Same as above & Same as above & Bright red blood from rectum \\
\hline IIA & Same as above & $\begin{array}{l}\text { Intestinal dilation, ileus, } \\
\text { pneumatosis }\end{array}$ & $\begin{array}{l}\text { Grossly bloody stools, } \\
\text { prominent abdominal } \\
\text { distention, absent bowel } \\
\text { sounds }\end{array}$ \\
\hline IIB & $\begin{array}{l}\text { Mild metabolic } \\
\text { acidosis and mild } \\
\text { thrombocytopenia }\end{array}$ & $\begin{array}{l}\text { Widespread pneumatosis, } \\
\text { ascites, portal-venous gas }\end{array}$ & $\begin{array}{l}\text {, Abdominal wall edema with } \\
\text { palpable loops and tenderness }\end{array}$ \\
\hline IIIA & $\begin{array}{l}\text { Mixed acidosis, } \\
\text { oliguria, hypotension, } \\
\text { coagulopathy }\end{array}$ & $\begin{array}{l}\text { Definite ascites, no free } \\
\text { air }\end{array}$ & $\begin{array}{l}\text { Generalized peritonitis, } \\
\text { abdominal wall edema, } \\
\text { erythema and induration }\end{array}$ \\
\hline IIIB & $\begin{array}{l}\text { Shock, deterioration in } \\
\text { laboratory values and } \\
\text { vital signs }\end{array}$ & Pneumoperitoneum & Same as stage IIIA \\
\hline
\end{tabular}

Adapted from Walsh and Kliegman ${ }^{23}$ 
TABLE 2. Baseline characteristics

A. Medical NEC vs. operative/fatal NEC

\begin{tabular}{|c|c|c|c|c|}
\hline & Medical NEC $(n=12)$ & $\begin{array}{l}\text { Operative/ fatal } \\
\text { NEC }(n=17)\end{array}$ & Total & $\mathbf{P}$ \\
\hline $\begin{array}{l}\text { Gestational age } \\
\text { (days)* }\end{array}$ & $\begin{array}{l}32+4(26+2- \\
38+2)\end{array}$ & $\begin{array}{l}29+3(27+2- \\
32+6)\end{array}$ & $\begin{array}{l}30+5(26+2- \\
38+2)\end{array}$ & $<0.05$ \\
\hline $\begin{array}{l}\text { Birth weight (grams) } \\
*\end{array}$ & $1465(860-1960)$ & $\begin{array}{l}1288(1000- \\
1738)\end{array}$ & $1400(860-1960)$ & 0.15 \\
\hline Sex & $7 \mathrm{M}(58 \%)$ & $4 \mathrm{M}(24 \%)$ & $11 \mathrm{M}(38 \%)$ & 0.12 \\
\hline \multicolumn{5}{|l|}{ B. NEC II vs. NEC III } \\
\hline & NEC II $(n=13)$ & NEC III $(n=16)$ & Total & $\mathbf{P}$ \\
\hline $\begin{array}{l}\text { Gestational age } \\
\text { (days)* }\end{array}$ & $\begin{array}{l}32+4(26+2- \\
38+2)\end{array}$ & $\begin{array}{l}29+1(27+2- \\
32+6)\end{array}$ & $\begin{array}{l}30+5(26+2- \\
38+2)\end{array}$ & $<0.05$ \\
\hline $\begin{array}{l}\text { Birth weight (grams) } \\
\star\end{array}$ & $1465(860-1960)$ & $\begin{array}{l}1220(1000- \\
1738)\end{array}$ & $1400(860-1960)$ & 0.17 \\
\hline Sex & $7 \mathrm{M}(54 \%)$ & $4 \mathrm{M}(25 \%)$ & $11 \mathrm{M}(38 \%)$ & 0.14 \\
\hline
\end{tabular}

* Data are presented as median (range)

TABLE 3. Values of IFABP, calprotectin, CRP and WBC at diagnosis

A. Medical NEC vs. operative/fatal NEC

\begin{tabular}{|c|c|c|c|}
\hline & Medical NEC $(n=12)$ & Operative/ fatal NEC $(n=17)$ & $\mathbf{P}$ \\
\hline I-FABP (pg/ml) & $5,500(300-142,000)$ & $5,000(1,100-1,712,000)$ & 0.51 \\
\hline $\begin{array}{l}\text { Fecal calprotectin } \\
\text { ( } \mu \mathrm{g} / \mathrm{g} \text { feces) }\end{array}$ & $375(146-848)$ & $479(108-684)$ & 0.87 \\
\hline CRP (mg/l) & $52.0(7.0-267)$ & $108(1.0-245)$ & 0.47 \\
\hline WBC $\left(\times 10^{9}\right.$ cells $\left./ \mathrm{I}\right)$ & $11.8(3.4-18.1)$ & $6.4(3.0-22.1)$ & 0.09 \\
\hline \multicolumn{4}{|l|}{ B. NEC II vs. NEC III } \\
\hline & NEC II $(n=13)$ & NEC III (n=16) & $\mathbf{P}$ \\
\hline I-FABP (pg/ml) & $4,800(300-142,000)$ & $11,100(1,100-1,712,000)$ & 0.41 \\
\hline $\begin{array}{l}\text { Fecal calprotectin } \\
\text { ( } \mu g / g \text { feces) }\end{array}$ & $378(317-848)$ & $495(108-684)$ & 0.75 \\
\hline CRP (mg/l) & $53.5(7.0-267)$ & $128.5(1.0-245)$ & 0.51 \\
\hline WBC $\left(\times 10^{9}\right.$ cells $\left./ \mathrm{I}\right)$ & $10.5(3.4-18.1)$ & $7.1(3.0-22.1)$ & 0.19 \\
\hline
\end{tabular}

Data are presented as median (range)

Fifteen patients were operated (all underwent laparotomy with intestinal resection), 9 on the day of diagnosis. The other 6 patients underwent surgery on median day 3 [2 - 29] (late surgery); 2 on day 2, 2 on day 3, one on day 7 and one 
on day 29. Indication for surgery was NEC in all cases (5 perforation, 10 clinical deterioration), and mortality (5 infants) was always related to NEC or its sequelae, which was confirmed by a pathologist specialized in pediatric gastroenterology. Two patients died without being operated. Of the 6 patients undergoing late surgery, the indication for surgery was clinical deterioration.

\section{Urinary I-FABP}

Urinary I-FABP levels were not statistically different between the operative/fatal NEC group $(5,000[1,100-1,712] \mathrm{pg} / \mathrm{ml})$ and the medical NEC group $(5,500$ $[300-142,000] \mathrm{pg} / \mathrm{ml}), p=0.51$, Table $3 \mathrm{~A}$. Similar results were found in the NEC III group $(11,100[1,100-1,712,000] \mathrm{pg} / \mathrm{ml})$ compared with the NEC II group $(4,800$ [300 - 142,000] pg/ml), $p=0.41$, Table 3B.

To investigate whether urinary I-FABP levels increased between time of diagnosis (D) and time of surgery, samples taken at two days (S-2) and one day (S-1) prior to surgery were analyzed. However, there were no significant differences.
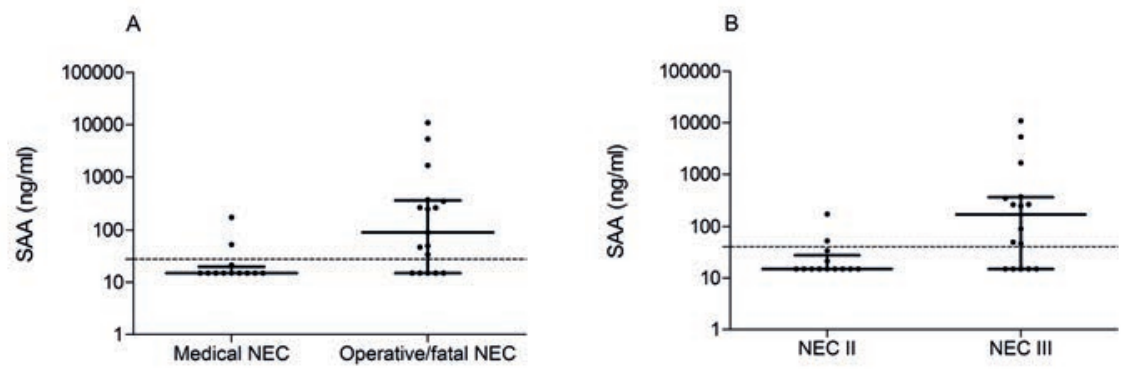

FIGURE 1. A. Urinary SAA levels at diagnosis (D) in the medical NEC group compared with the operative/fatal NEC group. The dotted line represents the ideal cut-off value of $27.8 \mathrm{ng} / \mathrm{ml}$. B. Urinary SAA levels at diagnosis (D) in the NEC stage II group compared with the NEC stage III group. The dotted line represents the ideal cut-off value of $40.7 \mathrm{ng} / \mathrm{ml}$.

\section{Urinary SAA}

Urinary SAA levels were significantly higher in the operative/fatal NEC group (90.8 [15.0 - 10,925] ng/ml) compared with the medical NEC group (15.0 [15.0 - 176] $\mathrm{ng} / \mathrm{ml}$ ), $p=0.007$ (Figure 1A). Test characteristics are summarized in Table 4.

Equivalent results were found in the NEC III group (172 [15.0 - 10,925] ng/ml) compared with the NEC II group (15.0 [15.0 - 167] ng/ml), $p=0.01$ (Figure 1B). 


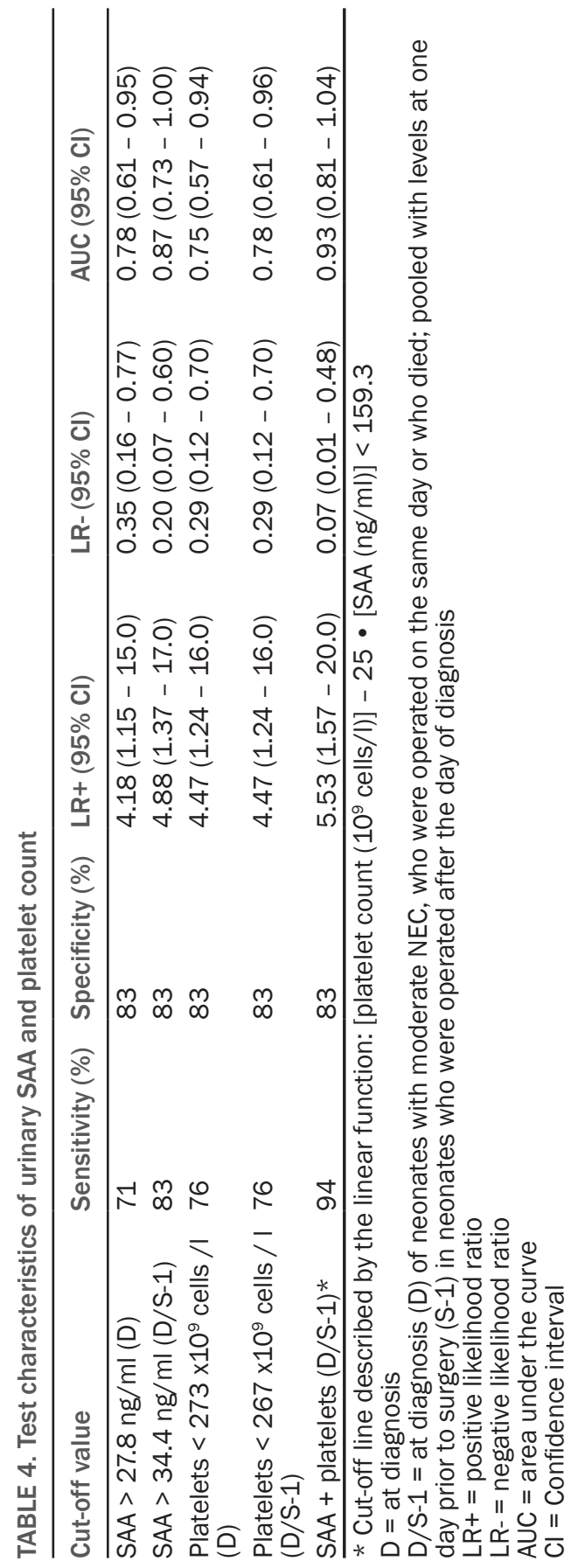




\section{Urinary SAA preoperatively}

To investigate whether urinary SAA levels increased between time of diagnosis (D) and time of surgery, samples taken at two days (S-2) and one day (S-1) prior to surgery were analyzed. In the six neonates undergoing surgery after the day of diagnosis, urinary SAA levels did not change in the period between diagnosis (15.0 $[15.0-28.0] \mathrm{ng} / \mathrm{ml})$ and S-2 (35.2 [15.0 - 1,718] ng/ml, $p=0.66)$. SAA levels tended to be higher at S-1 compared with SAA levels at diagnosis (respectively, 15.0 [15.0 - 28.0] ng/ml and 138 [15.0 - 3,647] ng/ml, $p=0.06$ ) although not reaching statistical significance, possibly caused by the small subgroup size (Figure 2A). At S-1, 4 out of 6 neonates had elevated urinary SAA levels, above the threshold of $27.8 \mathrm{ng} / \mathrm{ml}$.
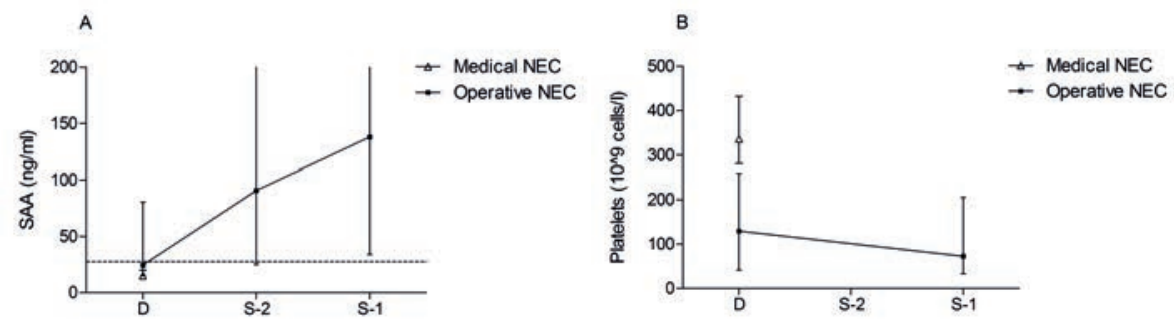

FIGURE 2. A. Development of urinary SAA levels in patients undergoing surgery 2 or more days after diagnosis. D: day of diagnosis, S-2: 2 days prior to surgery; S-1: 1 day prior to surgery. A cut-off value of $27.8 \mathrm{ng} / \mathrm{ml}$ is depicted by the dotted line. B. Development of platelet levels in patients undergoing surgery 2 or more days after diagnosis. D: day of diagnosis, S-2: 2 days prior to surgery; S-1: 1 day prior to surgery.

To assess whether the predictive value for surgery of urinary SAA changed with ongoing disease, urinary SAA levels at one day prior to surgery (S-1) were pooled with urinary SAA levels at diagnosis (D) of the neonates operated on the same day and neonates that eventually died of NEC. The area under the ROC curve in differentiating operative/fatal NEC from medical NEC increased to 0.87 , test characteristics are summarized in Table 4). Similar results were found in differentiating the NEC III group from the NEC II group (data not shown). 


\section{Urinary C3a and C5a}

Urinary $\mathrm{C} 3 \mathrm{a}$ and/or C5a levels were only detectable in 6 patients. Remarkably, these neonates were in the operative/fatal NEC and NEC III groups, however no reliable statistical differences could be calculated as complement activation products were not detectable in the majority of urine samples.

To investigate whether urinary C3a and C5a levels increased between time of diagnosis (D) and time of surgery, samples taken at two days (S-2) and one day (S-1) prior to surgery were analyzed. C3a levels increased in one patient prior to surgery and decreased in another patient prior to surgery. C5a levels were undetectable in samples at S-2 and S-1.

\section{Fecal calprotectin}

Fecal calprotectin levels analyzed in 16/29 patients who produced stools at diagnosis were not statistically different between the operative/fatal NEC group

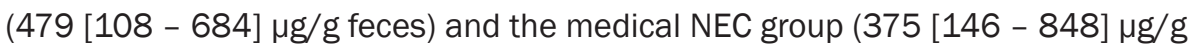
feces, $p=0.87$ ). Similar results were found for NEC stage III versus NEC stage II, Table 3.

\section{Conventional blood laboratory tests}

Values of C-reactive protein (CRP), white blood cell count (WBC), and platelets at the day of NEC diagnosis and one day prior to surgery were obtained from the patients' medical records. No statistical differences between moderate and severe NEC were found for CRP and WBC, Table 3. At diagnosis, platelets were significantly decreased in operative/fatal NEC (204 [8 - 654] x10 cells per liter $)$ compared with medical NEC (336 [71 - 583] x109 cells per liter, $p=0.02)$, and in NEC III (222 [8 - 654] x10 cells per liter) compared with NEC II (328 [71 - 583], $p=0.05$ ). Low platelet count yielded acceptable diagnostic accuracy in detecting severe NEC (test characterstics are summarized in Table 4). Similar results were found for NEC stage III versus NEC stage II.

Platelet levels in neonates operated several days after diagnosis dropped significantly from the day of diagnosis ( 130 [ 8 - 654] $\times 10^{9}$ cells per liter) to the day prior to operation (73 [8 - 411], $p=0.03$ ), Figure 2B. Platelet levels at one day prior to surgery (S-1) of neonates operated several days after diagnosis were pooled with platelet levels at diagnosis (D) of the neonates operated on the same day and neonates that eventually died of NEC. The area under the ROC curve in 
differentiating operative/fatal NEC from medical NEC did not change: 0.78 (test characteristics in Table 4). Results for NEC stage III versus NEC stage II were similar.

\section{Combination of urinary SAA and platelet count in the detection of severe NEC}

A logistic regression analysis approach was used to analyze the combination of urinary SAA with platelet count. For this analysis, the pooled dataset ( $D+S-1)$ was used. A cut-off line was calculated with area under the ROC curve, 0.93 (other test characteristics are described in Table 4), thereby increasing diagnostic accuracy compared to single marker measurement of urinary SAA or platelet count. The cut-off line was described by the linear function: [platelet count ( $10^{9}$ cells/I)] - 25 $x[S A A(n g / m l)]=159.3$; which means that a positive test is obtained when any combination of SAA and platelet levels in this formula results in a value $<159.3$ (Figure 3).

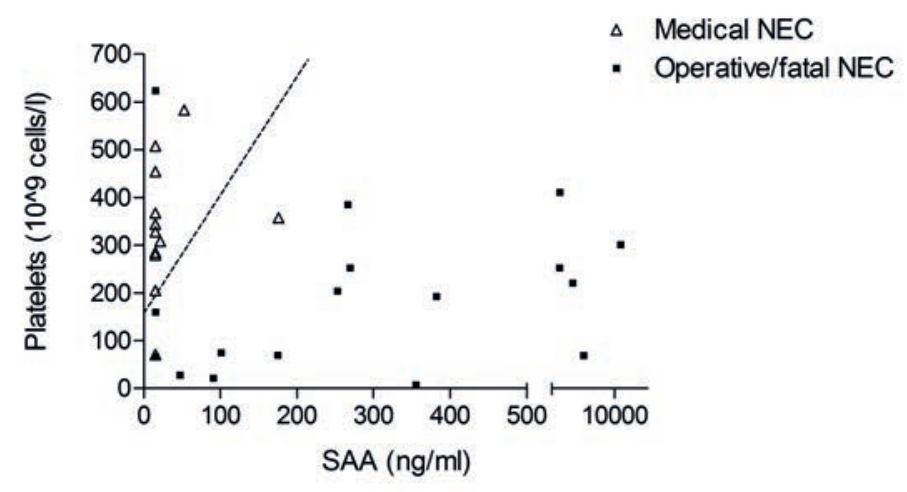

FIGURE 3. Combination of urinary SAA levels and platelet count in neonates with operative/fatal NEC (squares) and those with medically treated NEC (triangles) at diagnosis and one day prior to surgery in six neonates operated more than two days following diagnosis. The ideal cut-off line for differentiating between medical and operative/fatal NEC is depicted by the dotted line.

\section{Control values of markers}

Control values of the urinary markers I-FABP, SAA, C3a and C5a and the fecal marker calprotectin were measured in 20 premature neonates admitted for prematurity and/or small for gestational age. The control values are listed in Table 
5. Urinary SAA levels were not above the lower detection limit in all premature control neonates. C3a and C5a levels were only above the lower detection limit in respectively two controls and one control.

TABLE 5. Control values of urinary and fecal markers

\begin{tabular}{lll}
\hline & Median (range) & Mean $(\mathrm{SD})$ \\
\hline I-FABP $(\mathrm{pg} / \mathrm{ml})$ & $365(174-924)$ & $441(234)$ \\
$\mathrm{SAA}(\mathrm{ng} / \mathrm{ml})$ & No samples above lower detection limit of $15 \mathrm{ng} / \mathrm{ml}$ \\
$\mathrm{C} 3 \mathrm{a}(\mathrm{pg} / \mathrm{ml})$ & $250(250-1570) *$ & \\
C5a $(\mathrm{pg} / \mathrm{ml})$ & $1250(1250-1473) * *$ & \\
Calprotectin $(\mu \mathrm{g} / \mathrm{g}$ feces) & $68(31-335)$ & $110(104)$ \\
\hline
\end{tabular}

* No samples except two above lower detection limit

** No samples except one above lower detection limit

\section{Discussion}

This study shows that urinary levels of serum amyloid A (SAA) are significantly elevated in neonates with severe NEC defined as operative, fatal or stage III NEC compared with those with mild NEC, defined as medical or stage II NEC. Non-invasive measurement of SAA discriminates severe NEC from milder cases, especially in progressing disease, in which cases surgery was ultimately chosen for. Recently, Ng et al. reported that plasma SAA is a promising marker for the detection of NEC ${ }^{16}$. Furthermore, SAA in plasma has been reported to positively correlate with NEC stage ${ }^{4,24,25}$. In these studies, only the difference between Bell stages was investigated. Cetinkaya et al. (2010) showed that $80 \%$ of neonates with Bell stage I and $100 \%$ of neonates with Bell stage II/III had elevated plasma SAA levels ${ }^{4}$. This group also showed that plasma SAA levels were significantly higher in Bell stage III compared with Bell stage I at time of NEC diagnosis and on the days following diagnosis ${ }^{24}$. It may be questionable whether Bell stage I represents actual NEC, as no intestinal pneumatosis is present in this stage, however the results indicate an association between SAA levels and NEC stage. Eras et al. found elevated SAA levels in plasma in higher Bell stages at time of NEC diagnosis ${ }^{25}$. Urinary SAA has not been used previously to assess the severity of NEC. Non-invasive tests are favorable to minimize the risk of anemia ${ }^{26}$. Urinary measurement of SAA produces acceptable accuracy to detect severe NEC, and is able to differentiate between Bell stage II and Bell stage III. Serum platelet count markedly increases diagnostic accuracy, this procedure however necessitates blood sampling. In concordance with these results, low platelet count (less than 
$100 \times 10^{9}$ cells/I) has been described before to correlate with extension of NEC ${ }^{27}$ and laparotomy ${ }^{28}$. Platelet count alone resulted however in a post-test probability of $87 \%$ with a positive test and $29 \%$ with a negative test in the current study (pre-test probability of severe NEC, 59\%). When combined, platelet count and urinary SAA yielded a post-test probability of $89 \%$ with a positive test and $9 \%$ with a negative test, indicating that the negative predictive value of platelet count is improved considerably when SAA is added.

Selection of severe NEC cases is important to determine whether surgical intervention should be considered. While most studies focus on a difference between Bell stages, the current study also investigated a difference between medically treated NEC and surgically treated NEC/fatal NEC, which may be more accurate to assess the required treatment. Expectedly, analyses of both definitions showed equivalent results. It must be noted that severe NEC versus mild NEC is only a surrogate definition suggesting which infants with NEC should undergo surgical intervention. Clear criteria for surgical intervention, i.e. a gold standard, are lacking. In addition, the optimal type of surgery is uncertain, since both peritoneal drainage and laparotomy with resection of affected gut result in equal mortality rates and other clinical outcomes ${ }^{29}$.

The current study shows that early urinary SAA measurement correlates well with the clinical decision to perform surgery, and may therefore be able to assist the decision to perform surgery at an earlier time point. Future studies should focus on the effectiveness of early intervention as this matter has not yet been clarified. The median gestational age in the current study of 30+5 was higher compared to a recent study by $\mathrm{Ng}$ et al.: $28+3^{30}$, as was median birth weight: $1400 \mathrm{~g}$ compared to $1140 \mathrm{~g}^{30}$. The external validity of the current findings should therefore be verified in other hospitals, preferably in a multi-centered study design.

Analyses of other inflammatory markers in the current study showed no statistical differences between mild and severe NEC. However, the presence of elevated complement activation products ( $\mathrm{C} 3 \mathrm{a}$ and $\mathrm{C} 5 \mathrm{a}$ ) was limited to the severe NEC group, supporting the idea of an excessive inflammatory response in this group. Urinary measurement of $\mathrm{C} 3 \mathrm{a}$ and $\mathrm{C} 5 \mathrm{a}$ is not clinically relevant as these markers lacked diagnostic accuracy. Local inflammation as reflected by fecal calprotectin levels was not different in the severe NEC group compared with the mild NEC group. Moreover, no differences in intestinal epithelial cell damage reflected by I-FABP were observed between the groups. It can be hypothesized that severe NEC is characterized by increased systemic inflammation rather than 
increased mucosal breakdown and intestinal cell damage. It should be noted that fecal testing in NEC is not preferable as timing of bowel movements in preterm infants is unreliable and many patients present with (septic) ileus. In an earlier study by our group, urinary I-FABP levels accurately discriminated NEC from other diseases in neonates clinically suspected of NEC ${ }^{12}$. Therefore urinary I-FABP may be used to accurately diagnose NEC, while urinary SAA may help to guide the best treatment at an early stage of the disease, as an adjunct to clinical signs and symptoms. Serum platelet count may increase the accuracy of the SAA measurement.

A limitation of the study is the rather small group size. However to the best of our knowledge, this is one of the largest NEC cohorts, since definite NEC (Bell stage II and III) has a low incidence. Furthermore, this study represents two qualified pediatric surgery centers. Some remarks on the interpretation of the results of the current study must be made. First, urinary SAA measurement does not provide a $100 \%$ accuracy in selecting severe NEC cases. In the current cohort, with the given likelihood ratios and severe NEC prevalence of $59 \%$, the post-test probability is $87 \%$ ( $89 \%$ when platelet count is added) after a positive test and $22 \%$ ( $9 \%$ when platelet count is added) after a negative test, meaning that urinary SAA measurement cannot completely rule out nor identify severe NEC. Second, all markers except the conventional inflammatory markers were measured after patient enrollment. Therefore, low platelet count could have been used to guide the clinical decision of performing surgery, and these findings should thus be taken with caution. Blinding of the clinician to conventional blood tests is however not ethical. Third, next to surgical NEC cases, infants who died from NEC without undergoing surgery were included in the severe NEC group as well. Since these were only two patients, separate statistical analysis was not possible. It remains to be determined whether these patients would have benefited from surgical intervention, which is only feasible in a prospective study. Fourth, clinical decisions cannot be based exclusively on a single or several biomarkers. However the use of biomarkers can be a useful tool when combined with clinical assessment, especially when clinical improvement or deterioration is doubted. 


\section{References}

1. Lin PW, Stoll BJ. Necrotising enterocolitis. Lancet 2006;368:1271-83.

2. Neu J, Walker WA. Necrotizing enterocolitis. N Engl J Med 2011;364:255-64.

3. Bisquera JA, Cooper TR, Berseth CL. Impact of necrotizing enterocolitis on length of stay and hospital charges in very low birth weight infants. Pediatrics 2002;109:423-8.

4. Cetinkaya M, Ozkan $\mathrm{H}$, Koksal $\mathrm{N}$, et al. The efficacy of serial serum amyloid $\mathrm{A}$ measurements for diagnosis and follow-up of necrotizing enterocolitis in premature infants. Pediatr Surg Int 2010;26:835-41.

5. Edelson MB, Sonnino RE, Bagwell CE, et al. Plasma intestinal fatty acid binding protein in neonates with necrotizing enterocolitis: a pilot study. J Pediatr Surg 1999;34:14537.

6. Aydemir C, Dilli D, Oguz SS, et al. Serum intestinal fatty acid binding protein level for early diagnosis and prediction of severity of necrotizing enterocolitis. Early Hum Dev 2011;87:659-61.

7. Khoo AK, Hall NJ, Alexander $\mathrm{N}$, et al. Plasma soluble e-selectin in necrotising enterocolitis. Eur J Pediatr Surg 2008;18:419-22.

8. Tayman $\mathrm{C}$, Tonbul A, Kahveci $\mathrm{H}$, et al. C5a, a complement activation product, is a useful marker in predicting the severity of necrotizing enterocolitis. Tohoku J Exp Med 2011;224:143-50.

9. Morecroft JA, Spitz L, Hamilton PA, et al. Plasma interleukin-6 and tumour necrosis factor levels as predictors of disease severity and outcome in necrotizing enterocolitis. J Pediatr Surg 1994;29:798-800.

10. Smoller BR, Kruskall MS. Phlebotomy for diagnostic laboratory tests in adults. Pattern of use and effect on transfusion requirements. N Engl J Med 1986;314:1233-5.

11. Lin JC, Strauss RG, Kulhavy JC, et al. Phlebotomy overdraw in the neonatal intensive care nursery. Pediatrics 2000;106:E19.

12. Thuijls G, Derikx JP, van Wijck K, et al. Non-invasive markers for early diagnosis and determination of the severity of necrotizing enterocolitis. Ann Surg 2010;251:1174-80.

13. Evennett NJ, Hall NJ, Pierro A, et al. Urinary intestinal fatty acid-binding protein concentration predicts extent of disease in necrotizing enterocolitis. J Pediatr Surg 2010;45:735-40.

14. Malle E, Steinmetz A, Raynes JG. Serum amyloid A (SAA): an acute phase protein and apolipoprotein. Atherosclerosis 1993;102:131-46.

15. Meek RL, Eriksen N, Benditt EP. Serum amyloid A in the mouse. Sites of uptake and mRNA expression. Am J Pathol 1989;135:411-9.

16. Ng PC, Ang IL, Chiu RW, et al. Host-response biomarkers for diagnosis of lateonset septicemia and necrotizing enterocolitis in preterm infants. J Clin Invest 2010;120:2989-3000.

17. Carroll D, Corfield A, Spicer R, et al. Faecal calprotectin concentrations and diagnosis of necrotising enterocolitis. Lancet 2003;361:310-1.

18. Fagerhol MK. Calprotectin, a faecal marker of organic gastrointestinal abnormality. Lancet 2000;356:1783-4. 
19. Schlapbach $\sqcup$, Thiel $\mathrm{S}$, Kessler $\mathrm{U}$, et al. Congenital $\mathrm{H}$-ficolin deficiency in premature infants with severe necrotising enterocolitis. Gut 2010;60:1438-9.

20. Bell MJ, Ternberg JL, Feigin RD, et al. Neonatal necrotizing enterocolitis. Therapeutic decisions based upon clinical staging. Ann Surg 1978;187:1-7.

21. van der Sluijs Veer G, van den Hoven B, Russel MG, et al. Time-resolved fluorimetric immunoassay of calprotectin: technical and clinical aspects in diagnosis of inflammatory bowel diseases. Clin Chem Lab Med 2006;44:292-8.

22. Bossuyt PM, Reitsma JB, Bruns DE, et al. The STARD statement for reporting studies of diagnostic accuracy: explanation and elaboration. Clin Chem 2003;49:7-18.

23. Walsh MC, Kliegman RM. Necrotizing enterocolitis: treatment based on staging criteria. Pediatr Clin North Am 1986;33:179-201.

24. Cetinkaya M, Ozkan $\mathrm{H}$, Koksal $\mathrm{N}$, et al. Comparison of the efficacy of serum amyloid A, C-reactive protein, and procalcitonin in the diagnosis and follow-up of necrotizing enterocolitis in premature infants. J Pediatr Surg 2011;46:1482-9.

25. Eras Z, Oguz S, Dizdar EA, et al. Serum amyloid-A levels in neonatal necrotizing enterocolitis. J Clin Lab Anal 2011;25:233-7.

26. Strauss RG. Neonatal anemia: pathophysiology and treatment. Immunol Invest 1995;24:341-51.

27. Ververidis M, Kiely EM, Spitz L, et al. The clinical significance of thrombocytopenia in neonates with necrotizing enterocolitis. Journal of pediatric surgery 2001;36:799803.

28. Kenton AB, O'Donovan D, Cass DL, et al. Severe thrombocytopenia predicts outcome in neonates with necrotizing enterocolitis. J Perinatol 2005;25:14-20.

29. Moss RL, Dimmitt RA, Barnhart DC, et al. Laparotomy versus peritoneal drainage for necrotizing enterocolitis and perforation. N Engl J Med 2006;354:2225-34.

30. Ng EW, Poon TC, Lam HS, et al. Gut-Associated Biomarkers L-FABP, I-FABP, and TFF3 and LIT Score for Diagnosis of Surgical Necrotizing Enterocolitis in Preterm Infants. Ann Surg 2013;258:1111-8. 


Chapter 12. Non-invasive measurement of intestinal epithelial damage at time of re-feeding can predict clinical outcome after necrotizing enterocolitis

Kostan W. Reisinger, Joep P.M. Derikx, Geertje Thuijls, David C. van der Zee, Hens A.A. Brouwers, Annemarie A. van Bijnen, Tim G.A.M. Wolfs, L.W. Ernest van Heurn, Wim A. Buurman, Boris W. Kramer. Pediatric Research 2013. 73(2):209-13 


\begin{abstract}
Background

Reintroduction of enteral nutrition in neonates with necrotizing enterocolitis (NEC) should take place when the gut is ready. Too early start of oral feeding might lead to disease relapse, whereas prolonged discontinuation of enteral nutrition is associated with impaired gut function and parenteral nutrition-related complications. This study evaluates whether non-invasive urinary measurement of intestinal Fatty Acid Binding Protein (I-FABP) at time of re-feeding can predict clinical outcome in neonates with NEC.
\end{abstract}

\title{
Methods
}

Urinary I-FABP concentrations were measured in 21 infants with NEC just before reintroducing enteral nutrition. Poor outcome was defined as unsuccessful reintroduction of enteral feeding, (re)operation for NEC, or death related to NEC after reintroduction of enteral feeding.

\section{Results}

Median urinary I-FABP levels in neonates with poor outcome $(n=5)$ were significantly higher compared to I-FABP levels in neonates with good outcome $(\mathrm{n}=16)(\mathrm{p}<0.01)$. A clinically significant cut-off value of $963 \mathrm{pg} / \mathrm{ml}$ was found to discriminate infants with poor outcome from those with good outcome (sensitivity $80 \%$, specificity $94 \%)$.

\section{Conclusion}

Non-invasive urinary I-FABP measurement at time of re-feeding differentiates neonates with poor outcome from neonates with good outcome in NEC. Urinary I-FABP measurement may therefore be helpful in the timing of enteral feeding in neonates with NEC. 


\section{Introduction}

Necrotizing enterocolitis (NEC) is a severe gastrointestinal disease with high morbidity and mortality (20-40\%), affecting predominantly premature neonates 1. The treatment of choice for infants suffering from mild to moderate NEC includes cardiorespiratory support if necessary, broad-spectrum antibiotics, and discontinuation of enteral feeding ${ }^{1-3}$. Surgical intervention is considered in patients with a perforation or advanced NEC that does not improve with conservative treatment ${ }^{2,4}$.

After the initial treatment of NEC, there is no common opinion regarding the time when enteral feeding can be reintroduced. There are no clinical, radiological and laboratory parameters to determine when re-feeding of the infant is safe. Refeeding at a very early stage has been suggested to lead to recurrence of the disease ${ }^{5}$, whereas prolonged discontinuation of enteral feeding can worsen gut function ${ }^{6}$ and impair growth. Moreover, prolonged use of parenteral nutrition is associated with catheter-related septicemia and longer duration of hospital stay ${ }^{7}$.

In a recent study, we demonstrated that urinary intestinal Fatty Acid Binding Protein (I-FABP) is a suitable non-invasive marker to confirm the diagnosis of NEC in patients with early symptoms of NEC ${ }^{8}$. I-FABP, a small (14-15 kDa) cytosolic protein, is solely present in mature epithelial cells of the small and large intestine 9. It is immediately released into the circulation upon loss of cell membrane integrity and passes the glomerular filter with a fractional renal excretion of $28 \%$ and a half life time of 11 minutes, which makes it detectable in urine ${ }^{10}$. Therefore, urinary I-FABP levels accurately reflect the actual extent of intestinal epithelial cell damage, which makes I-FABP an indicator of disease progression ${ }^{8}$. While initially elevated, urinary I-FABP levels decrease when disease severity diminishes ${ }^{11}$. We therefore hypothesize that I-FABP levels may be useful to determine the ideal time for reintroducing enteral feeding.

In this study, we measured urinary I-FABP concentrations in infants who were previously treated for NEC at the time oral feeding was introduced and evaluated the outcome of these children. 


\section{Methods}

\section{Patients, feeding protocol and sample collection}

21 consecutive infants, in whom enteral feeding was reintroduced after treatment for NEC in the neonatal intensive care units at Maastricht University Medical Centre and Wilhelmina Children's Hospital in Utrecht, between July 2005 and August 2010 were included in the study. NEC was diagnosed with the current gold standard of abdominal X-ray showing pneumatosis intestinalis (Bell stage II or higher) ${ }^{2} .20$ consecutive premature infants (matched with NEC infants for gestational age at birth) admitted to the neonatal intensive care unit at Maastricht University Medical Centre and without gastrointestinal symptoms were also included for evaluation of control values. Indication for admission into the neonatal intensive care unit was small for gestational age or prematurity in all cases. In these premature neonates serving as controls, a urine sample was collected. A second control group of 9 neonates without intestinal injury was included, in whom enteral feeding was stopped and restarted. Indications for cessation of enteral feedings were suspicion of NEC (eventually diagnosed as sepsis) or laparotomy for malformation or atresia without significant intestinal epithelial cell damage. Written consent was obtained from both parents, and the study was conducted with approval from local medical ethical committees, and according to the revised version of the Declaration of Helsinki (October 2008, Seoul). The principles of good clinical practice (GCP) were followed during this study.

Feeding was restarted in patients who improved clinically: spontaneously breathing patients without intestinal pneumatosis on abdominal X-ray, without abdominal distention, with peristalsis and a soft abdomen. This decision was made by the attending neonatologist, who was independent of the investigators. Enteral feeding consisted of formula feeding, breast milk or a combination of both, and was increased with a maximum of $20 \mathrm{ml} / \mathrm{kg} /$ day.

Urine was obtained daily by placing a dental cotton roll (Henry Schein, Almere, the Netherlands) in the diaper of the neonate. Once the roll was filled with urine, it was placed in a sterile $5 \mathrm{~mL}$ syringe (Becton Dickinson, Oxford, United Kingdom), the urine was pressed into Micronic tubes (Micronic B.V., Lelystad, the Netherlands) and stored at $-20^{\circ} \mathrm{C}$ until analysis. Urine samples were collected at time of diagnosis (Diag), just before reintroduction of enteral feeding (EF), and the day after reintroduction of enteral feeding $(E F+1)$. 
All patient materials were collected in a prospective manner, however the analyses were performed by one person who was blinded to clinical diagnoses after completion of patient inclusion. Attending clinicians were therefore not aware of I-FABP levels when deciding to restart enteral feeding.

\section{Urinary I-FABP measurement}

Urinary I-FABP was measured using an in-house enzyme-linked immunosorbent assay (ELISA) that selectively detects human I-FABP (standard: 10-1280 pg/ml). Values are expressed as concentration $(\mathrm{pg} / \mathrm{ml})$ and ratio $(\mathrm{pg} / \mathrm{nmol}$ creatinine) of I-FABP $(\mathrm{pg} / \mathrm{ml})$ to creatinine $(\mu \mathrm{mol} / \mathrm{l})$, to compensate for variations in urine concentration.

\section{Statistical analyses}

Normality was tested by Kolmogorov-Smirnov. Mann-Whitney $U$ test was used for between-groups comparison for continuous data. Dichotomous variables were compared using Fisher exact test. All data are presented as median and range. Outcome was defined as poor clinical outcome (unsuccessful introduction of enteral feeding (i.e. restarting total parenteral nutrition), (re)operation or death within 30 days after diagnosis of NEC) or good clinical outcome. To find a cut off point of urinary I-FABP levels at time of re-feeding that most accurately predicts outcome, a receiver operating characteristic (ROC) curve was drawn by plotting sensitivity against 1-specificity for all possible thresholds. Overall accuracy of urinary I-FABP to predict outcome was represented by area under the curve (AUC). The best cut off point was defined as the cut off point with maximum sum of sensitivity and specificity, after taking the range of normal values into account. Statistical analyses were performed with Prism 5.0 for Windows (GraphPad Software Inc. San Diego, CA). STARD statement for reporting studies of diagnostic accuracy was used in this study ${ }^{12}$. 


\section{Results}

\section{Patients and outcome}

Twenty-one neonates were included in the study, of whom 12 were males. The patient characteristics are listed in Table 1. There were no significant differences between groups in gestational age, birth weight, or sex, nor in blood laboratory tests (CRP, leucocytes, platelets) at time of re-feeding (Table 1).

TABLE 1. Baseline characteristics and conventional laboratory tests

\begin{tabular}{lllll}
\hline & Poor outcome & Good outcome & Total & P \\
\hline Gestational age (days) & $207(191-268)$ & $224(184-242)$ & $224(184-268)$ & 0.55 \\
$\begin{array}{l}\text { Birth weight } \\
\text { (grams) }\end{array}$ & $1300(1185-$ & $1463(860-2106)$ & $1460(860-2960)$ & 0.78 \\
Sex & $2960)$ & & & \\
Surgery before re- & $3 \mathrm{M} 2 \mathrm{~F}$ & $9 \mathrm{M} 7 \mathrm{~F}$ & $12 \mathrm{M} 9 \mathrm{~F}$ & 1.00 \\
feeding & $3 / 5(60 \%)$ & $6 / 16(37.5 \%)$ & $9 / 21(42.9 \%)$ & 0.61 \\
CRP $(\mathrm{mg} / \mathrm{I})$ & $1.8(1.0-5.8)$ & $40(1.0-93)$ & $5.8(1.0-93)$ & 0.11 \\
Leucocytes $\left(\times 10^{9}\right.$ cells/I) $14.5(10.3-16.5)$ & $10.1(8.0-20.1)$ & $10.4(8.0-20.1)$ & 0.16 \\
Platelets $\left(x 10^{9}\right.$ cells/l) & $309(96-892)$ & $120(29-296)$ & $191.5(29-892)$ & 0.11 \\
\hline
\end{tabular}

Data are presented as median (range)

$\mathrm{CRP}=\mathrm{C}$-reactive protein

Five of the 21 infants with NEC had a poor clinical outcome, and reintroduction of enteral feeding was unsuccessful (total parenteral nutrition was restarted). Four out of these five received an operation or re-operation on NEC within 30 days after reintroduction of enteral feeding, and the $5^{\text {th }}$ child died within 30 days after

TABLE 2. Specification of patients with poor clinical outcome

\begin{tabular}{llll}
\hline & $\begin{array}{l}\text { Discontinuation of enteral } \\
\text { feeding after re-feeding }\end{array}$ & $\begin{array}{l}\text { Operation after re- } \\
\text { feeding }\end{array}$ & Death after re-feeding \\
\hline Patient A & Yes & Yes $^{\mathrm{a}}$ & No \\
Patient B & Yes & No & Yes $^{\mathrm{b}}$ \\
Patient C & Yes & Yes & Yes $^{\mathrm{c}}$ \\
Patient D & Yes & Yes $^{\mathrm{a}}$ & Yes $^{\mathrm{d}}$ \\
Patient E & Yes & Yes $^{\mathrm{a}}$ & No \\
\hline
\end{tabular}

Columns are ordered from left to right by increasing severity of adverse outcome.

a Second operation for NEC. Active NEC was found in all cases.

${ }^{b}$ Died within 30 days after diagnosis of NEC

${ }^{\circ}$ Died 71 days after diagnosis of NEC, cause of death: persistent neonatal seizures

d Died 162 days after diagnosis of NEC, cause of death: treatment discontinued because of severe neurological damage 
reintroduction of oral feeding (Table 2). Three of the four infants operated for NEC after re-feeding had been operated on earlier, 0-2 days after the initial diagnosis. In all four cases, NEC was the indication for operation, which was confirmed with histopathology. The other 16 neonates recovered from NEC after enteral feeding was restarted, without the need for surgery (good clinical outcome group).

The median period of time between birth and NEC diagnosis was 9 (4 - 30) days in the good outcome group and $5(2-18)$ days in the poor outcome group, $p=0.28$. The median period of time that infants received no feeding by mouth after NEC diagnosis was $9(6-19)$ days in the group with good outcome and 10 $(6-14)$ in the group with poor outcome, $p=0.50$. In the poor outcome group, the period of re-feeding lasted for $8(3-34)$ days. These results are shown in Table 3. The median period of time until full enteral feeding was achieved was 9 days (no difference between groups).

TABLE 3. Time course

\begin{tabular}{lllll}
\hline & \multicolumn{2}{l}{ Poor outcome } & Good outcome Total & P \\
\hline Birth - diagnosis (days) & $5(2-18)$ & $9(4-30)$ & $9(2-30)$ & 0.28 \\
$\begin{array}{l}\text { Diagnosis - re- feeding (days) } \\
\text { Re-feeding - second discontinuation } 8(3-34)\end{array}$ & $10(6-14)$ & $9(6-19)$ & $9(6-19)$ & 0.50 \\
of enteral feeding (days) & & & \\
\hline
\end{tabular}

Data are presented as median (range)

High urinary I-FABP levels at time of re-feeding correlates with poor outcome

Median urinary I-FABP:creatinine ratio (I-FABP corrected for urine concentration) just before reintroduction of enteral feeding was significantly higher in neonates with poor outcome $(6.00(0.53-247.10) \mathrm{pg} / \mathrm{nmol}$ creatinine) as compared with neonates with good outcome $(0.38(0.03-1.41) \mathrm{pg} / \mathrm{nmol}$ creatinine $), p<0.01$. To distinguish neonates with poor outcome from neonates with good outcome, an ideal cut off value of $2.23 \mathrm{pg} / \mathrm{nmol}$ creatinine was found with a sensitivity of $80 \%$ and a specificity of $100 \%$. This resulted in a positive likelihood ratio (LR) of $>12.8$ and a negative LR of 0.2 .

Similar data were obtained when I-FABP was not corrected for urine concentration (Figure 1). This analysis led to an ideal cut off value of $963 \mathrm{pg} / \mathrm{ml}$, with a sensitivity of $80 \%$ and a specificity of $94 \%$; a positive LR of 12.8 and a negative LR of 0.21 . The area under the curve (AUC) for urinary I-FABP in differentiating neonates 


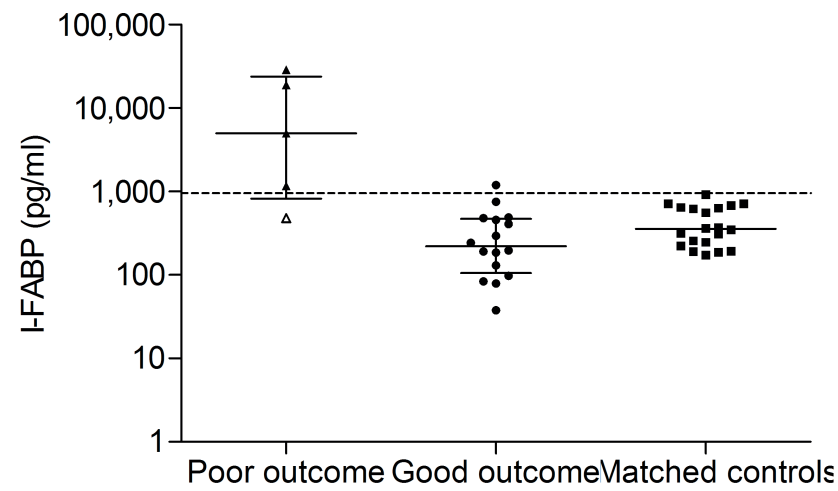

FIGURE 1. Urinary I-FABP levels of on day of reintroduction of enteral feeding, median 9 days after diagnosis of NEC. Data are expressed as median I-FABP levels with interquartile ranges. A cut-off value of $963 \mathrm{pg} / \mathrm{ml}$ is marked by the dotted line. I-FABP levels of controls matched for gestational age are depicted. $\boldsymbol{\Delta}$ operated after re-feeding; $\bullet$ not operated after re-feeding; $\Delta$ died after re-feeding; matched controls.

with poor outcome from neonates with good outcome was 0.93 (95\% confidence interval $(\mathrm{Cl}) 0.78-1.00)$ for I-FABP corrected for urine concentration, and 0.95 $(95 \% \mathrm{Cl} 0.86$ - 1.00) for I-FABP not corrected for urine concentration.

Median urinary I-FABP for 20 premature neonates without gastrointestinal symptoms (matched controls) was $358(174-924) \mathrm{pg} / \mathrm{ml}$ (Figure 1).

Relative difference in urinary I-FABP levels from diagnosis to time of re-feeding as a predictor of poor outcome

Another approach to predicting the outcome of reintroduction of enteral feeding would be to measure the relative reduction of intestinal damage as reflected by the differences in I-FABP levels from the moment of diagnosis to the moment of oral re-feeding. The relative difference $(\triangle \mathrm{Rel})$ in urinary I-FABP from diagnosis (Diag) to reintroduction of enteral feeding $(E F)$ was calculated as follows:

$\Delta \mathrm{ReI}_{\text {Diag-EF }}=\left((\mathrm{I}-\mathrm{FABP})_{\mathrm{EF}}-(\mathrm{I}-\mathrm{FABP})_{\text {Diag }}\right) /(\mathrm{I}-\mathrm{FABP})_{\text {Diag }} * 100 \%$, in which $(\mathrm{I}-\mathrm{FABP})$ is the concentration of I-FABP (not corrected for urine concentration) $(\mathrm{pg} / \mathrm{ml}) . \Delta \mathrm{Rel}_{\text {Diag-EF }}$ in neonates with good outcome $(-100 \%(-100 \%--22 \%))$ was significantly lower than $\Delta \mathrm{Rel}_{\text {Diag-EF }}$ in neonates with poor outcome $(-66 \%(-87 \%-+1137 \%)), p<0.05$ (Figure 2). To distinguish neonates with poor outcome from neonates with good outcome, an ideal cut off value of minus $90 \%$ was found with a sensitivity of $100 \%$ and a specificity of $75 \%$. This resulted in a positive LR of 4.00 and a negative LR of 


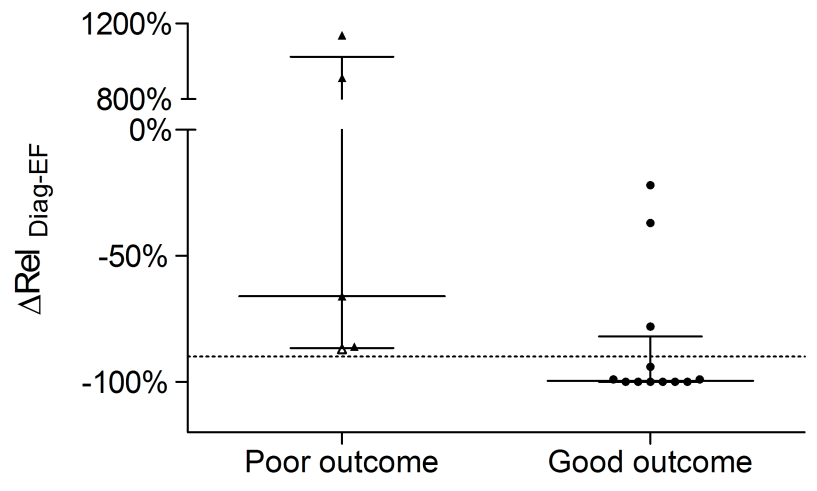

FIGURE 2. Relative differences with median and interquartile ranges of urinary I-FABP from diagnosis to reintroduction of enteral feeding ( $\triangle$ Rel Diag-EF). A cut-off value of minus $90 \%$ is marked by the dotted line. $\boldsymbol{\Delta}$ operated after re-feeding; • not operated after re-feeding; $\Delta$ died after re-feeding.

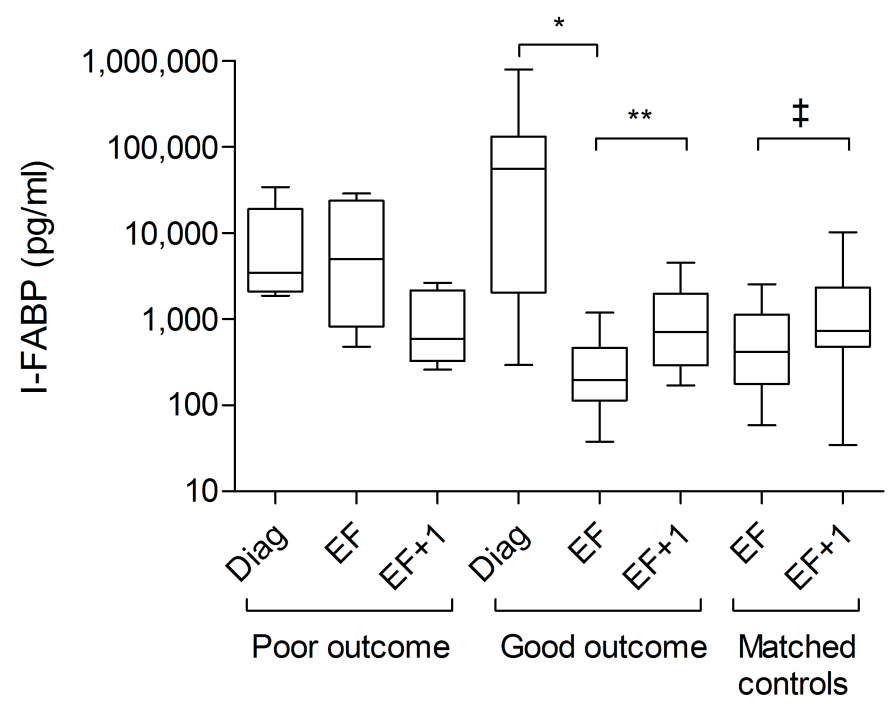

FIGURE 3. Urinary I-FABP levels on the day of diagnosis, the day of reintroduction of enteral feeding and one day after reintroduction of enteral feeding. * $p=0.002 ; * * p=0.04 ; \ddagger p=0.03$.

0.00. The area under the curve for $\Delta$ Rel $_{\text {Diag:EF }}$ in differentiating neonates with poor outcome from neonates with good outcome was 0.87 (95\% Cl 0.69 - 1.00) (ROC curve not shown). Actual urinary I-FABP levels at time of diagnosis are depicted 
in Figure 3. There was no significant difference in urinary I-FABP levels at time of diagnosis between the groups. In conclusion, the former analysis based on actual values at the moment of re-feeding produced better data.

\section{Kinetics of I-FABP following reintroduction of enteral feeding}

In the analyses, both I-FABP corrected for urine concentration and I-FABP uncorrected for urine concentration were used.

Next we analyzed whether differences could be detected between neonates with good clinical outcome and those with poor clinical outcome early during feeding. To this end we compared data at EF with data on EF+1. Analysis of the data revealed a significant increase in urinary I-FABP from EF $(0.38(0.03-1.41)$ $\mathrm{pg} / \mathrm{nmol}$ creatinine) to $\mathrm{EF}+1$ (1.41 (0.53 - 2.74) pg/nmol creatinine, $p<0.05)$ in the neonates with good outcome. In contrast, neonates with poor outcome showed no increase from EF to EF+1.

Similar data were obtained in case I-FABP was not corrected for urine concentration; a significant increase in urinary I-FABP from EF (197 (37.7 - 1,196) $\mathrm{pg} / \mathrm{ml})$ to $E F+1(712(170-4,537) \mathrm{pg} / \mathrm{ml}, p=0.04)$ was observed in the group with good outcome (Figure 3). This increase (EF, 421 (59 - 2,553); EF+1, 733 (35 - 10,278), $p=0.03$, Figure 3) was also observed in the control group of 9 infants without intestinal injury in whom feeding was discontinued and reintroduced, indicating a physiological rather than a pathological response.

\section{Discussion}

One of the challenges in the treatment of the infant with NEC is to choose the best moment to reintroduce enteral feeding. Although no benefit has been shown for delayed ${ }^{13}$ or slow ${ }^{14}$ introduction of enteral feeding in the prevention of NEC, evidence is lacking on the length of the period between the treatment of NEC and the moment that enteral feeding can be restarted safely. Although a period of 7-10 days of discontinuation of enteral feeding has been recommended ${ }^{15}$, faster reintroduction after NEC seems feasible in some cases. In a study by Bohnhorst et al. ${ }^{7}$, enteral feeding was restarted as early as 4 days after diagnosis of NEC when portal vein gas was absent on ultrasound. These infants showed less catheterrelated sepsis and a shorter hospital stay as compared with historical controls in which enteral feeding was reinitiated on day $10^{7}$. Moreover, return to enteral 
feeding may reduce the development of villus atrophy ${ }^{16}$. We consider that daily measurement of urinary I-FABP could help to identify infants who can be safely returned to oral feeding.

The results of this study show that urinary I-FABP is a suitable non-invasive marker to determine which infants with NEC in whom enteral feeding is restarted are at risk for a poor outcome, in terms of mortality or re-operation. In addition to clinical assessment, urinary I-FABP measurement can therefore be a helpful tool for the neonatologist in deciding which infants can be re-fed safely in the course of NEC, especially when clinical improvement is doubted. At the moment, no laboratory markers exist to confirm this decision. In the population studied, a cutoff value at the time of oral re-feeding of $963 \mathrm{pg} / \mathrm{ml}$ could discriminate neonates with poor outcome from those with good outcome, with a sensitivity of $80 \%$ and a specificity of $94 \%$. Whether clinical deterioration could have been avoided by delaying reintroduction of enteral feeding, or whether clinical deterioration can directly be caused by onset of re-feeding cannot be determined by these data. Further study in a large number of patients is needed to evaluate the cut-off value of $963 \mathrm{pg} / \mathrm{ml}$ and its clinical usefulness.

Remarkably, I-FABP levels increased early during feeding in the group with good outcome and in a control group without NEC or other severe intestinal injury. This may be a result of a physiological response of the gut when challenged by enteral feeding. In a piglet model, rapid enterocyte turnover and mucosal structural changes were observed 8 hours after introduction of enteral feeding ${ }^{17}$. Unexpectedly, I-FABP levels seemed to decrease early during re-feeding in the group with poor outcome, however the difference was not statistically significant. This could be a consequence of reduction of enterocyte mass as described earlier in severe mucositis induced by myeloablative conditioning ${ }^{18}$. However, the size of this group is relatively small, which is an inevitable problem, as many of the children who die of NEC die shortly after NEC diagnosis without the possibility to restart enteral feeding.

In conclusion, when I-FABP levels were above $963 \mathrm{pg} / \mathrm{ml}$ following clinical progression which resulted in reintroducing enteral feeding, there was a high chance of clinical deterioration in this NEC population. Urinary I-FABP measurement may therefore be helpful in the timing of enteral feeding in neonates with NEC. However, this hypothesis has to be validated in a prospective trial, with different treatment strategies determined on the basis of urinary I-FABP measurement that serves as an addition to clinical judgment. 


\section{References}

1. Lin PW, Stoll BJ. Necrotising enterocolitis. Lancet 2006;368:1271-83.

2. Bell MJ, Ternberg JL, Feigin RD, et al. Neonatal necrotizing enterocolitis. Therapeutic decisions based upon clinical staging. Ann Surg 1978;187:1-7.

3. Neu J. Neonatal necrotizing enterocolitis: an update. Acta Paediatr Suppl 2005;94:1005.

4. Lee JS, Polin RA. Treatment and prevention of necrotizing enterocolitis. Semin Neonatol 2003;8:449-59.

5. Berseth CL. Feeding strategies and necrotizing enterocolitis. Curr Opin Pediatr 2005;17:170-3.

6. Lin PW, Nasr TR, Stoll BJ. Necrotizing enterocolitis: recent scientific advances in pathophysiology and prevention. Semin Perinatol 2008;32:70-82.

7. Bohnhorst B, Muller S, Dordelmann M, et al. Early feeding after necrotizing enterocolitis in preterm infants. J Pediatr 2003;143:484-7.

8. Thuijls G, Derikx JP, van Wijck K, et al. Non-invasive markers for early diagnosis and determination of the severity of necrotizing enterocolitis. Ann Surg 2010;251:1174-80.

9. Veerkamp JH, Paulussen RJ, Peeters RA, et al. Detection, tissue distribution and (sub) cellular localization of fatty acid-binding protein types. Mol Cell Biochem 1990;98:118.

10. van de Poll MC, Derikx JP, Buurman WA, et al. Liver manipulation causes hepatocyte injury and precedes systemic inflammation in patients undergoing liver resection. World J Surg 2007;31:2033-8.

11. Evennett NJ, Hall NJ, Pierro A, et al. Urinary intestinal fatty acid-binding protein concentration predicts extent of disease in necrotizing enterocolitis. J Pediatr Surg 2010;45:735-40.

12. Bossuyt PM, Reitsma JB, Bruns DE, et al. The STARD statement for reporting studies of diagnostic accuracy: explanation and elaboration. Clin Chem 2003;49:7-18.

13. Morgan J, Young L, McGuire W. Delayed introduction of progressive enteral feeds to prevent necrotising enterocolitis in very low birth weight infants. Cochrane Database Syst Rev 2011:CD001970.

14. Morgan J, Young L, McGuire W. Slow advancement of enteral feed volumes to prevent necrotising enterocolitis in very low birth weight infants. Cochrane Database Syst Rev 2011:CD001241.

15. Neu J, Walker WA. Necrotizing enterocolitis. N Engl J Med 2011;364:255-64.

16. Kliegman RM, Walker WA, Yolken RH. Necrotizing enterocolitis: research agenda for a disease of unknown etiology and pathogenesis. Pediatr Res 1993;34:701-8.

17. Oste M, Van Haver E, Thymann T, et al. Formula induces intestinal apoptosis in preterm pigs within a few hours of feeding. JPEN J Parenter Enteral Nutr 2010;34:271-9.

18. Derikx JP, Blijlevens NM, Donnelly JP, et al. Loss of enterocyte mass is accompanied by diminished turnover of enterocytes after myeloablative therapy in haematopoietic stem-cell transplant recipients. Ann Oncol 2009;20:337-42. 


Chapter 13. Summary and discussion 


\section{Introduction}

Major gastrointestinal surgery has considerable impact on the patients' physical condition and carries significant risk on postoperative complications, although in many gastrointestinal disorders, surgery is the only curative option. Preservation of gut function is therefore essential in major surgery. Inadequate wound healing of the gut can lead to the most severe form of gut function disruption: leakage of luminal contents to the sterile milieu of the abdominal, mediastinal and pelvic cavities, inducing severe inflammation, sepsis and finally death. Efforts should therefore be taken to prevent intestinal cell damage, attenuate inflammation and improve intestinal wound healing perioperatively. Furthermore, biomarkers of intestinal cell damage and inflammation may be suitable tools to detect failure of intestinal wound healing and complications at an early time-point.

In this thesis, it was hypothesized that especially in vulnerable patients with gastrointestinal disorders, complications are likely to develop. Therefore, novel strategies to prevent postoperative complications were investigated in adult patients with specific emphasize on the high-risk elderly patient. Both a clinical trial and observational studies were described to gain new insight in intestinal damage, intestinal wound healing and inflammation during major gastrointestinal surgery and the effects of sarcopenia, nutritional compromise and frailty on postoperative recovery. Next, plasma markers of intestinal cell damage and inflammation to diagnose postoperative complications in colorectal surgery were evaluated. Finally, premature neonates were investigated, as these patients represent an extremely vulnerable population. Necrotizing enterocolitis (NEC) is the most common and severe gastrointestinal emergency in these patients. To improve outcome in NEC, the pathophysiology was investigated, aiming two important predisposing factors: gut immaturity and formula feeding. In addition, it was investigated whether specific biomarkers of intestinal damage and gut inflammation could be used in the diagnosis, treatment and follow-up of NEC.

\section{Part 1. Pathophysiologic aspects of complications after adult gastrointestinal surgery}

The first aim of this thesis focused on prevention of complications after major gastrointestinal surgery. Major surgery accompanied by systemic hypotension and blood loss is thought to lead to redistribution of blood to preserve the vital organs (brain and heart) at the expense of the splanchnic circulation ${ }^{1,2}$. Since 
tissue perfusion and oxygenation are needed to ensure adequate wound healing, intestinal hypoperfusion during colorectal surgery may lead to impaired intestinal wound healing, specifically anastomotic healing. Furthermore, hypoperfusion can impair intestinal barrier function, leading to increased susceptibility to bacterial translocation and sepsis. Keeping the splanchnic circulation at a constantly sufficient level is challenging, particularly because specific clinical read-outs as well as treatment options to selectively improve splanchnic perfusion are lacking. Theoretically, perfusion is dependent on two variables: flow and pressure. Splanchnic flow is often further reduced when vasoactive agents are used, inducing splanchnic vasoconstriction.

Doppler guided goal-directed fluid therapy increases intestinal perfusion in colorectal surgery

In chapter 2, a randomized clinical trial was described in which systemic blood flow was optimized by fluid administration based on Doppler-based stroke volume measurements. Maintaining mean arterial pressures above $65 \mathrm{mmHg}$ resulted in reduced intestinal damage in a previous study involving major non-abdominal surgery ${ }^{3}$. This effect of hemodynamic optimization was not found in patients undergoing colorectal surgery since no significant intestinal damage was observed in both the control and intervention groups as measured by plasma intestinal fatty acid binding protein (I-FABP) levels, a highly specific marker for enterocyte damage 4. However, a significant improvement of gastrointestinal perfusion was observed by measuring gastric mucosal $\mathrm{pCO}_{2}$ levels. It should be noted that this method provides an indication of global intestinal perfusion and may therefore miss disturbances at a more microcirculatory level that are sufficient to cause damaged villus tips. It may well be that selected patients, i.e. those who have substantial tissue oxygenation problems, benefit from increasing splanchnic flow. A trial in line with this hypothesis shows that patients with normal oxygen consumption levels do not benefit from hemodynamic optimization in terms of complications, time to ready for discharge, and length of hospital stay, while the opposite effect may be true in aerobically unfit patients ${ }^{5}$. This underlines the importance of an adequate selection of patients who can benefit from a certain approach and those who do not, addressed as personalized healthcare. 


\section{Cyclooxygenase-2 is essential for colorectal anastomotic healing}

In chapter 3, the harmful effects of non-steroidal anti-inflammatory drugs (NSAIDs) on intestinal wound healing became apparent. Although a correlation between NSAID use and anastomotic leakage after colorectal surgery had already been established in patients, the results from this rodent model showed that the enzyme cyclooxygenase-2 (COX-2), which is inhibited by NSAIDs, is essential to ensure adequate intestinal wound healing. Mice genetically lacking COX-2 displayed impaired intestinal wound healing. In addition, it was shown that the mechanism behind COX-2 inhibition and anastomotic leakage possibly acts through compromised neovascularization. Not only should the perioperative use of NSAIDs be avoided in patients undergoing colorectal surgery, patients with mutations in the COX-2 gene may be vulnerable to impaired intestinal wound healing and the subsequent risk of complications. Selection and personalized treatment of these patients should therefore be investigated. Personalized healthcare using gene variation and tumor mutations is increasingly being applied in oncology. Indeed, COX-2 has been implicated in this context. Long-term use of NSAIDs increases survival in patients with mutated-PIK3CA colorectal cancer (a mutation leading to increased COX-2 expression), but not in those with wild-type PIK3CA ${ }^{6}$. The effect was most pronounced in patients with PIK3CA mutations and COX-2 expression in the tumor, pointing at the important role of COX-2 in intestinal homeostasis. This particular subgroup could therefore benefit for longterm survival from additional NSAID treatment. Perioperatively, such an approach may as well be used in patients with COX-2 mutations, with the marked difference that NSAIDs have negative effects. This is however not inconsistent, as intestinal vascularization and growth are of paramount importance when recovering from colorectal surgery, while the opposite is true when treating a tumor. Short-term therapeutic use of prostaglandins, the products of COX-2, needs therefore further exploration in colorectal surgery, particularly in patients with mutations in the COX-2 pathway, since animals lacking COX-2 showed better anastomotic healing when prostaglandin E2 was administered.

Functional compromise is associated with complications in gastrointestal surgery

Other fundamentals for individualized healthcare were explored in chapters 4, 5 and 6 . These studies investigated the high-risk elderly patients undergoing gastrointestinal surgery. Elderly patients are known to have decreased physiologic 
reserves, affecting the ability to adequately recover from profound therapeutic hits such as major surgery. In most developed countries, the population is ageing, making the treatment of elderly patients a challenge of growing importance. Since the exact mechanisms of increased vulnerability to postoperative complications in the elderly are not known, some aspects coming with age were investigated in chapter 4. Frailty, sarcopenia and nutritional compromise were not convincingly predictive of postoperative morbidity and mortality when analyzed separately. However, the combination of these factors, which could be addressed as a measure of generalized functional compromise, was associated with unfavorable postoperative outcome, supporting the hypothesis that increased risk for postoperative complications in the elderly is multifactorial. This functional compromise was predominantly associated with sepsis and mortality. Therefore it was investigated in chapter 5 whether skeletal muscle wasting or sarcopenia correlated with increased inflammation in response to colorectal surgery, since sarcopenia is an important hallmark of decreased physiologic reserves in elderly patients. Indeed sarcopenic patients showed increased postoperative inflammation, in concordance with previous data ${ }^{7}$. These results together suggest that sarcopenia incorporates an increased inflammatory response leading to increased risk of postoperative sepsis and death. This highlights the fact that sarcopenic patients represent a distinct population in which caution should be taken. In chapter 6 , the knowledge on the role of sarcopenia in gastrointestinal surgery was extended, showing that patients lost skeletal muscle mass when receiving chemoradiation before esophagectomy. Although neoadjuvant chemoradiotherapy may positively influence cancer survival and recurrence rates ${ }^{8}$, one should bear in mind that these patients' physical condition is weakened, predisposing to high risk of negative sequelae. The amount of skeletal muscle mass lost was actually predictive of postoperative mortality in these patients. However, no direct correlation between sarcopenia and postoperative complications was found, which was not in line with the results in chapter 4. Moreover, the amount of muscle mass lost only predicted mortality in patients with advanced tumors, who were more likely to be sarcopenic. These results suggest that the decline of physiologic reserves reflected as sarcopenia plays a role in the development of postoperative morbidity and mortality, although this effect is not generalizable, that is, dependent on patient population and type and stage of the tumor. It would be interesting to know whether muscle mass decay is also observed in patients receiving neoadjuvant therapy for rectal cancer, and whether baseline muscle 
mass levels differ across cancer types, to define specific risk populations and adapt preventive and treatment protocols in a personalized medicine-manner. In addition, future research should aim at comparing histology of muscle biopsies and parameters of muscle function, with computed tomography (CT). The measurements of muscle mass and sarcopenia in chapters 4 till 6 were done using CT. It is however not known whether these findings are also reflected by muscle histology, such as type I/type II fiber ratio and satellite cell content. Moreover, low muscle mass may not necessarily be accompanied by low muscle function. In the future, prehabilitation protocols should be investigated, aiming at improvement of muscle mass, function and histology, possibly leading to improved postoperative recovery.

The CT based method was described before by Prado and colleagues ${ }^{9}$. This method is easy to perform, as a staging CT scan is usually performed in gastrointestinal cancer necessitating surgery and measurements can be done using the open source software OsiriX®. The core measurement in this method is the total skeletal muscle area on the third vertebral (L3) level. In all studies described in this thesis, predefined sex-specific cutoff values for sarcopenia were used, which were identified in obese cancer patients based on optimal stratification for mortality ${ }^{9}$. Although this is a well-validated method to assess muscle mass, some remarks must be made. First, sarcopenia is a more comprehensive syndrome than just low muscle mass. Functional parameters such as gait speed and handgrip strength should be taken into account as well to diagnose sarcopenia properly. Second, the predefined L3 cutoff values, although accepted in the most recent consensus on sarcopenia ${ }^{10}$, may not be applicable to all populations. A more appropriate way to define cutoff values may be to determine normal values in a large cohort representing the general population, for this method does not stratify for mortality. Keeping these limitations in mind, measurements of physical compromise such as sarcopenia can be used to predict postoperative complications at preoperative assessment. New options for preoperative treatment of sarcopenia and concurrent frailty should be investigated. Both nutritional and muscle exercise interventions have been proposed to counteract sarcopenia, with the best effects when both strategies are combined, which elicits the greatest anabolic response. Effectiveness of such a dual approach on postoperative outcomes has not been investigated thus far. This will also lead to optimal use of CT scans that are now 
only performed for diagnostic reasons. Cost-effectiveness of CT scans, and the responsible use of expertise and medical resources will be improved, which is in accordance with the CanMEDS framework ${ }^{11}$.

\section{Conclusion}

In conclusion, these results provide new knowledge on preventive measures to reduce complications of gastrointestinal surgery. A number of unresolved issues remain. Splanchnic perfusion can be improved using optimization of the systemic circulation, although this is not sufficient to attenuate intestinal cell damage. Whether specific populations at risk for complications due to circulatory and tissue oxygenation problems could benefit from such an approach remains to be determined. Furthermore, a fundamental role of COX-2 dysfunction in impaired intestinal wound healing was discovered. The question raised by this finding is whether patients undergoing gastrointestinal surgery may benefit from prostaglandin supplementation, and specifically those who have low intrinsic COX2 levels or activity. Finally, physical deterioration apparent as sarcopenia, frailty and/or nutritional compromise was associated with unfavorable postoperative outcome, particularly in elderly patients and advanced disease. Complication risk can be predicted using measurements of physical compromise, and new studies should address whether methods to counteract sarcopenia lead to better physical performance and subsequent lower complication rates. Taken together, the described studies are highly suggestive that various determinants of postoperative morbidity exist across different subpopulations, supporting the need for further research to implement personalized treatment protocols.

\section{Part 2. Biomarkers of complications after adult gastrointestinal surgery}

The second aim of this thesis was to detect one of the most severe complications after colorectal surgery, anastomotic leakage, at an early time-point. To this end, specific markers of enterocyte damage and markers of inflammation were measured daily in plasma (chapter 7). Remarkably, the enterocyte specific markers did not show postoperative differences between patients with anastomotic leakage and those without. In addition, values were not elevated compared to healthy controls, indicating that enterocyte damage is limited in anastomotic leakage. Nonetheless, preoperative intestinal fatty acid binding protein (I-FABP) levels were elevated in 50 percent of patients ultimately developing anastomotic leakage. This points to 
some intrinsic process predisposing these patients to impaired intestinal wound healing. It would be interesting to know whether the mechanism underlying this increased baseline intestinal cell damage could be addressed to hypoperfusion (chapter 2) or a genetic disruption in the COX-2 pathway (chapter 3). Again, the idea of personalized treatment is supported, as these patients may require nonstandard approaches, i.e. construction of a diverting ostomy, performing Hartmann's procedure or using the preoperative time-frame for prehabilitation programs. Furthermore, in chapter 7 high diagnostic accuracy was described when markers of generalized inflammation, C-reactive protein (CRP) and calprotectin, a neutrophil activation product, were measured at the third postoperative day. Postoperative CRP has been described before to detect anastomotic leakage ${ }^{12}$, but the current results show that diagnosis can be made both more accurately and earlier when calprotectin measurement is added. More importantly, diagnosis can be established three days earlier than clinical signs become apparent. This is of great relevance, because clinical symptoms generally develop when systemic dissemination of bacteria and toxic products is already present, which is a late stage of anastomotic leakage. Untimely recognition of anastomotic leakage may lead to avoidable sepsis with subsequent cardiorespiratory failure, intensive care unit admissions, and death.

\section{Conclusion}

Chapter 7 supports the hypothesis to develop differential approaches based on patient characteristics and risks. Evidence-based flowcharts are feasible and easy to use tools to implement personalized healthcare. Such a treatment algorithm based on the present findings is presented in chapter 7 , figure 4 . High preoperative I-FABP values can filter out half of patients who develop anastomotic leakage without false-positive results (sensitivity 50\%, with post-test probability $100 \%$ ), and for whom a different surgical approach can be considered. The best postoperative diagnostic accuracy is obtained when CRP and calprotectin are combined at postoperative day 3. CRP/calprotectin measurement on postoperative day 3 can therefore aid to determine which patients can be discharged safely and which patients should be monitored intensively. The next step would be to study treatment decision based on the preoperative I-FABP, postoperative CRP and calprotectin levels, and to investigate whether this leads to prevention and better management of anastomotic leakage after colorectal surgery. 


\section{Part 3. Pathophysiologic aspects of necrotizing enterocolitis (NEC)}

The third aim of this thesis was to characterize gut-specific biomarkers in the context of gut maturation to gain better understanding of the pathophysiology of NEC. As NEC is a disease primarily affecting premature neonates receiving formula feeding, it was investigated whether I-FABP levels were determined by these factors. To this end, neonates admitted to the intensive care unit without gastrointestinal symptoms were studied. In chapter 8, urinary I-FABP levels shortly after birth were measured in these children, demonstrating significant elevation in extremely premature neonates ( $<28$ weeks gestational age at birth) compared to moderately premature (28-36 weeks) and term (37-42 weeks) neonates. Previously, I-FABP distribution and release were evaluated during gestation in utero in fetal lambs, since the developmental biology of sheep is closest to the human development for most major organs including the gut ${ }^{13}$. Paradoxically, circulating I-FABP levels in fetal lambs increased with gestational age, which paralleled increasing I-FABP content in intestinal tissue. In addition, the epithelial expression pattern of I-FABP changed from a phenotype comprising predominantly I-FABP positive enterocytes in the crypts in extremely premature lambs to the phenotype similar to the human adult situation with I-FABP positive cells principally present at the villus tips. These findings are interesting for several reasons. First, urinary I-FABP may be useful as a marker of gut maturation, since its distribution and release patterns are gestational age dependent. Second, the discrepancy between circulating prenatal ovine I-FABP levels and those measured shortly after birth in the human situation support the hypothesis that some stimulus after birth, such as bacterial colonization or introduction of enteral feeding, induces damage to the premature intestine, predisposing to NEC development. Third, since circulating I-FABP levels are low in extremely premature lambs in utero, elevation of postnatal I-FABP levels may underestimate the actual intestinal damage. This is of importance when I-FABP is used to diagnose NEC in these children, and different cutoff values may be applicable across gestational ages. Formula feeding is the second most important risk factor for NEC. In chapter 9, urinary I-FABP levels were measured in premature infants receiving exclusively formula feeding or breast milk in the first weeks after birth. In breast fed infants, a significant increase of I-FABP levels towards normal values was observed. This probable physiologic increase was observed with a certain delay in babies who were formula fed. These data, together with the findings of chapter 8 , suggest that intestinal maturation is delayed in formula fed infants. This sheds new light 
on the beneficial effects of breast milk or breast milk substitutes. The search for breast milk alternatives is ongoing, aiming at growth factors, anti-inflammatory substances and prebiotics. In the future, I-FABP may be used as a clinical read-out to evaluate the effect of such strategies on intestinal maturation.

\section{Conclusion}

In conclusion, these studies shed new light on the clinical use of the enterocyte marker I-FABP. In addition to its accuracy to diagnose intestinal epithelial cell damage, which has been validated in several studies, I-FABP can be used to evaluate intestinal epithelial maturation in neonates. It has to be investigated whether patients with delayed or decreased maturation as measured by urinary or plasma I-FABP levels actually have increased risk to develop gut immaturity related complications, such as the development of NEC and feeding intolerances, and gut-derived sepsis.

\section{Part 4. Biomarkers of necrotizing enterocolitis (NEC)}

The final aim of this thesis was to evaluate the usefulness of noninvasive markers of intestinal damage and inflammation in the detection and follow-up of necrotizing enterocolitis (NEC), the most important gastrointestinal emergency in neonates, which often necessitates surgery. Early recognition of this disease is extremely important, as it may quickly progress to systemic disease and death. However, the clinical differentiation between NEC and other gastrointestinal disorders and sepsis in the early phase is difficult and clinical signs are nonspecific. Previously, urinary I-FABP was found to accurately discriminate between NEC and other disease in children who were clinically suspected of having NEC ${ }^{14,15}$. In chapter 10 , this prospective cohort was expanded and the combination of urinary I-FABP and calprotectin measured in feces yielded even increased accuracy to detect NEC, compared to I-FABP alone. In addition to the challenge to diagnose NEC, the decision to perform surgery in neonates is a delicate one, as surgery and anesthesia have profoundly negative effects on mortality rates and neurodevelopmental impairment ${ }^{16}$. In chapter 11 , the same markers were tested to predict severe NEC, in which neonates surgical resection of the affected gut is necessary. I-FABP and calprotectin were not suitable to this end, however the acute-phase protein serum amyloid A (SAA) showed a good predictive value, especially when combined with platelet count. Both systemic inflammation and thrombocytopenia 
are hallmarks of advanced NEC or Bell's stage III ${ }^{17}$. Apparently, the differentiation of NEC from other diseases is best reflected by gut-specific markers of damage and inflammation, while among NEC patients, the primary process indicating the need for surgery is systemic inflammation reflecting disease progression. As I-FABP and SAA were measured in urine and calprotecin was measured in feces, these analyses can be done noninvasively. This is an important advantage, as blood collection for diagnostic purposes is a major cause for anemia in neonates. Obviously, platelet count cannot be determined in noninvasively collected samples. A disadvantage of fecal calprotectin was the unavailability of fecal samples in a substantial proportion of children. Next, in chapter 12, it was investigated whether noninvasive measurement of I-FABP could be used in the follow-up after NEC episodes. The classical NEC treatment consists of discontinuation of enteral feeding, intravenous administration of broad-spectrum antibiotics and cardiopulmonary support. It is unknown when enteral feeding could be restarted, that is when the gut has recovered. In chapter 12 it was demonstrated that urinary measurement of I-FABP can aid in the decision to restart enteral feeding, since infants with high I-FABP levels at the time feeding was reintroduced were likely to develop unfavorable clinical outcome, i.e. relaparotomy or death.

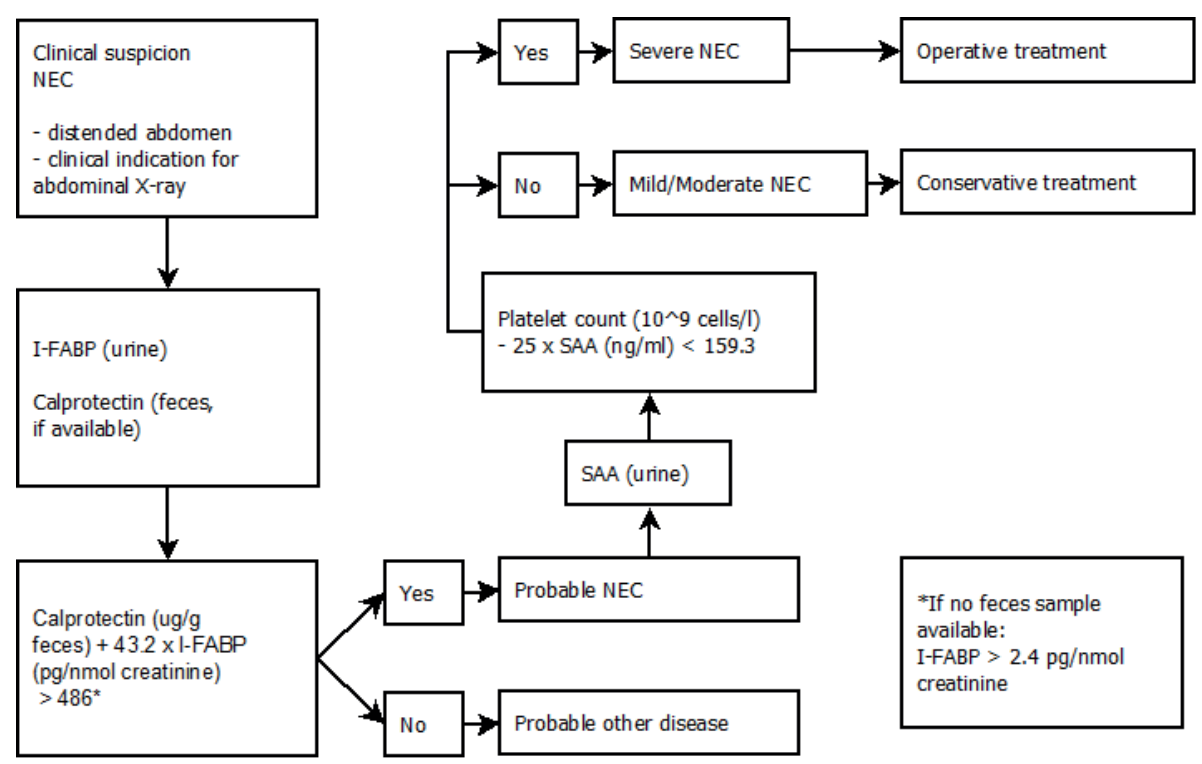

FIGURE 1. Flowchart of diagnosis, treatment and follow-up of NEC. 


\section{Conclusion}

Collectively, these data indicate that noninvasive measurement of markers of intestinal cell damage and inflammation, together with markers of systemic inflammation, can be used in the diagnosis, the decision to perform surgery and the decision to reintroduce enteral feeding in NEC. These studies are promising, and need to be verified in a phase IV trial before robust conclusions can be drawn. In this context, personalized treatment according to flowcharts seems practical. In figure 1, a flowchart based on the findings in this thesis is presented. Infants suspected of NEC by having a distended abdomen or in whom an abdominal X-ray is indicated, urine and feces (if available) must be collected. Initially, urinary I-FABP and fecal calprotectin are measured. If I-FABP and calprotectin are elevated, NEC diagnosis is highly likely and NEC treatment should be initiated. Next, elevated SAA and low platelet count can identify patients in whom surgical intervention is necessary, and which patients can be treated medically. Of note, during first assessment patients can have moderate NEC which develops into severe NEC later on. Therefore, urinary SAA and if available, platelet count, are monitored daily. Finally, during treatment, urinary I-FABP levels are monitored daily and if dropping below the cutoff value of $2.23 \mathrm{pg} / \mathrm{nmol}$ creatinine (chapter 12), reintroduction of enteral feeding is associated with successful outcome. 


\section{Main conclusion}

The central hypothesis of this thesis was that especially in vulnerable patients with gastrointestinal disorders, inadequate wound healing, postoperative morbidity and mortality are likely to develop. We showed that sarcopenia, nutritional compromise and frailty, which are parameters of vulnerability in elderly patients, are indeed associated with complications and mortality after gastrointestinal surgery. Suboptimal fluid status was correlated with decreased intestinal perfusion, which may increase the risk of complications, although a decrease of intestinal cell damage was not observed. Lack of the enzyme COX-2 predisposed the gut to impaired angiogenesis and wound healing, indicating that patients using COX-2 blocking analgesics or those with genetic COX-2 mutations are susceptible to postoperative morbidity. Using markers of inflammation and intestinal damage, postoperative complications could be accurately predicted. Preoperative increased intestinal cell damage was associated with postoperative complications, pointing to the vulnerability of these patients. In neonates, it was shown that prematurity and formula feeding were associated with impaired gut development, highlighting the vulnerability of these patients to develop gastrointestinal morbidity, particularly NEC. Markers of intestinal cell damage and inflammation were shown to assist in detecting infants with NEC at an early stage, detecting infants with NEC needing surgical intervention, and detecting infants with NEC in whom enteral feeding could be restarted. These data together support the hypothesis of this thesis. Moreover, it was shown that parameters of vulnerability can be useful in clinical practice, contributing to improved care in gastrointestinal surgery. 


\section{References}

1. Moore FA. The role of the gastrointestinal tract in postinjury multiple organ failure. Am J Surg 1999;178:449-53.

2. Derikx JP, van Waardenburg DA, Thuijls G, et al. New Insight in Loss of Gut Barrier during Major Non-Abdominal Surgery. PLoS One 2008;3:e3954.

3. Thuijls G, Derikx JP, de Kruijf M, et al. Preventing enterocyte damage by maintenance of mean arterial pressure during major nonabdominal surgery in children. Shock 2012;37:22-7.

4. Gollin G, Marks C, Marks WH. Intestinal fatty acid binding protein in serum and urine reflects early ischemic injury to the small bowel. Surgery 1993;113:545-51.

5. Challand C, Struthers R, Sneyd JR, et al. Randomized controlled trial of intraoperative goal-directed fluid therapy in aerobically fit and unfit patients having major colorectal surgery. Br J Anaesth 2012;108:53-62.

6. Liao X, Lochhead P, Nishihara R, et al. Aspirin use, tumor PIK3CA mutation, and colorectal-cancer survival. N Engl J Med 2012;367:1596-606.

7. Richards $\mathrm{CH}$, Roxburgh CS, MacMillan MT, et al. The relationships between body composition and the systemic inflammatory response in patients with primary operable colorectal cancer. PLoS One 2012;7:e41883.

8. Pennathur A, Gibson MK, Jobe BA, etal. Oesophageal carcinoma. Lancet 2013;381:40012.

9. Prado CM, Lieffers JR, McCargar $L$, et al. Prevalence and clinical implications of sarcopenic obesity in patients with solid tumours of the respiratory and gastrointestinal tracts: a population-based study. Lancet Oncol 2008;9:629-35.

10. Fearon K, Strasser F, Anker SD, et al. Definition and classification of cancer cachexia: an international consensus. Lancet Oncol 2011;12:489-95.

11. Frank JR, Danoff D. The CanMEDS initiative: implementing an outcomes-based framework of physician competencies. Med Teach 2007;29:642-7.

12. Warschkow R, Beutner U, Steffen T, et al. Safe and early discharge after colorectal surgery due to C-reactive protein: a diagnostic meta-analysis of 1832 patients. Ann Surg 2012;256:245-50.

13. Wolfs TG, Jellema RK, Turrisi G, et al. Inflammation-induced immune suppression of the fetus: a potential link between chorioamnionitis and postnatal early onset sepsis. J Matern Fetal Neonatal Med 2012;25 Suppl 1:8-11.

14. Derikx JP, Evennett NJ, Degraeuwe PL, et al. Urine based detection of intestinal mucosal cell damage in neonates with suspected necrotising enterocolitis. Gut 2007;56:14735.

15. Thuijls G, Derikx JP, van Wijck K, et al. Non-invasive markers for early diagnosis and determination of the severity of necrotizing enterocolitis. Ann Surg 2010;251:1174-80.

16. Neu J, Walker WA. Necrotizing enterocolitis. N Engl J Med 2011;364:255-64.

17. Bell MJ, Ternberg JL, Feigin RD, et al. Neonatal necrotizing enterocolitis. Therapeutic decisions based upon clinical staging. Ann Surg 1978;187:1-7. 


Valorization 
The findings in this thesis are not only relevant from a scientific point of view, but also carry social and economic value. As described in detail in chapter 13, several conclusions drawn from the different studies in this thesis form the basis for improvements in health care strategies. First, we have added to a growing body of evidence that widely used analgesics, the NSAIDs, should be banned from use in the context of gastrointestinal surgery, since this may lead to preventable complications and unnecessarily high health care costs. Patients undergoing major gastrointestinal surgery as well as the community paying health care costs may benefit from this advancement. In the same study, we have shown that administration of prostaglandin E2 may prevent complications in an experimental model of colonic surgery, however this might be mostly applicable to specific subsets of patients. Future research should reveal whether clinical application of prostaglandin E2 indeed decreases complication rates and thereby health care costs and the burden to society.

Next, we aimed at unraveling the phenotype of the vulnerable elderly patient undergoing major gastrointestinal surgery. This population specifically deserves attention as a burden to health care costs. The population of the Netherlands and many other countries is ageing, thereby increasingly demanding health care resources. Furthermore, due to therapeutic improvements over the past decades, high-impact therapies such as major surgery are applied to older, sicker and more vulnerable patients, while complication rates and thus health care costs are high in these patients. Since most research has focused on younger patients, improvements in the elderly are relatively limited. In this thesis, we have presented simple tools to identify patients who are at risk for developing complications. For the first time, we established an association between frailty, sarcopenia and malnutrition on one hand and complication rates after major gastrointestinal cancer surgery on the other. This is important scientific knowledge, which should be explored in further detail. In the near future we will perform a research project that aims to improve the peri-operative physical condition of gastric and colorectal cancer patients, to decrease susceptibility to postoperative complications. A dual modality approach using nutritional supplementation with essential amino acids and physical exercise on physical functioning after gastric and colorectal surgery will be investigated. Essential amino acids and physical exercise elicit the greatest anabolic response. Therefore this is hypothesized to counteract sarcopenia and 
malnutrition. To create the greatest societal support, patient associations will be involved to implement patient requests and to resolve practical issues with regard to the nutritional and physical interventions.

Obviously, the target group described in this thesis was comprised of vulnerable patients undergoing major gastrointestinal surgery. However, the results might be applicable to other patient populations as well. A nutritional support and physical exercise program as proposed above could be extrapolated to other vulnerable patient groups, such as patients undergoing chemotherapy and those recovering from severe trauma. The problem of vulnerable elderly patients is not limited to those undergoing surgery.

Another main research theme of this thesis was the accuracy and applicability of biomarkers in plasma, urine and stool. First, we showed interesting results on markers detecting complications after colorectal surgery. As these are observational data, we are aiming to study these markers in a larger, multicenter cohort and eventually to change therapeutic decision making to improve health care. Currently, we have initiated a comparable research project to study the same markers in gastric surgery, to broaden the target group of patients who can benefit from these results. In the second part of this thesis we described non-invasive markers to diagnose necrotizing enterocolitis (NEC) in premature neonates and to guide the decision to perform surgery and to reintroduce enteral feeding. In chapter 13, we presented a decision tree when NEC is clinically suspected. We are aiming to investigate whether clinical implementation in a nationwide study decreases morbidity and mortality in children with NEC. 

Samenvatting 


\section{Inleiding}

Dit proefschrift gaat over darmschade, inflammatie en wondgenezing rondom grote maag-darmchirurgie, met als doel nieuw inzicht te krijgen in het voorkomen, detecteren en behandelen van complicaties.

Grote maag-darmchirurgie is een belangrijke pijler in de behandeling van diverse aandoeningen aan het maag-darmkanaal; het brengt echter een aanzienlijk risico met zich mee op het ontwikkelen van complicaties. Zoals bij alle grote operaties en bij maag-darmchirurgie in het bijzonder, is het behoud van de darmfunctie essentieel. De functies van de darm zijn het opnemen van voedingsstoffen en het buitenhouden van schadelijke stoffen en micro-organismen. Als deze functies niet intact zijn, gaat dit ten koste van het herstel na een operatie. Hiervoor is namelijk adequate voeding nodig, evenals een effectieve barrière tegen micro-organismen. Bij maag-darmchirurgie wordt een stuk van de maag of darm weggenomen, waardoor een wond ontstaat. Slechte wondgenezing kan leiden tot lekkage van micro-organismen en zelfs darminhoud naar steriele lichaamsholten, zoals de buikholte. Dit kan leiden tot hevige inflammatie, sepsis en uiteindelijk overlijden. Het is daarom van essentieel belang dat mogelijkheden worden onderzocht om darmschade en inflammatie zo laag mogelijk te houden en de wondgenezing van de darm te verbeteren. Daarnaast kunnen markers van darmschade en inflammatie nuttig zijn om inadequate wondgenezing van de darm en zodoende complicaties op te sporen.

In dit proefschrift werd gezocht naar nieuwe strategieën om complicaties na maag-darmchirurgie te voorkomen. Dit werd gedaan aan de hand van een klinische trial en verschillende observationele studies. Vervolgens werd gekeken naar de diagnostische waarde van darmschademarkers en inflammatoire markers om complicaties op te sporen. Tenslotte werd onderzocht of markers van darmschade en inflammatie gebruikt konden worden in de diagnose, behandeling en vervolgbeleid bij grote maag-darmchirurgie bij kinderen. De centrale hypothese van dit proefschrift was dat met name bij kwetsbare patiënten de kans op complicaties na grote maag-darmchirurgie groot is en dat er specifieke aandacht moet komen voor deze kwetsbare patiënten. In de studies die in dit proefschrift werden beschreven, werd dan ook de nadruk gelegd op de kwetsbare oudere patiënt en zoals hierboven aangegeven de kwetsbare extreem jonge patiënt. 


\section{Deel 1. Nieuwe strategieën ter voorkoming van complicaties}

Het eerste doel van dit proefschrift was factoren en mechanismen te bestuderen die kunnen leiden tot darmschade, inflammatie en verminderde wondgenezing om zodoende nieuwe strategieën te kunnen bedenken om complicaties te verminderen.

Het optimaliseren van de vochthuishouding leidt tot een verbeterde doorbloeding van de darm

In hoofdstuk 2 werd onderzocht of het optimaliseren van de vochthuishouding leidde tot minder darmschade en verbeterde darmdoorbloeding. Dit was een klinische trial, waarbij patiënten die een dikke darmoperatie ondergingen, werden ingedeeld in een groep die de normale behandeling kreeg en een groep die de normale behandeling kreeg plus het optimaliseren van de vochthuishouding. In beide groepen werd geen darmschade gemeten. In de geoptimaliseerde groep werd echter wel een verbetering van de darmdoorbloeding gemeten. Geconcludeerd kan worden dat met name patiënten die doorbloedingsproblemen van de darm hebben, in de toekomst kunnen profiteren van vochtoptimalisatie. Van dergelijke doorbloedingsproblemen kan bijvoorbeeld sprake zijn in het kader van algehele zwakte of vaatproblematiek.

\section{COX-2 is essentieel voor adequate darmgenezing}

In hoofdstuk 3 werden de schadelijke effecten van veelgebruikte pijnstillers onder de loep genomen. De werking van deze pijnstillers, waar bijvoorbeeld diclofenac en aspirine deel van uitmaken, berust op remming van het eiwit cyclooxygenase-2 (COX-2). Wij toonden aan dat het gebruik van diclofenac tot een slechtere wondgenezing van de darm leidt en dat dit slechte neveneffect inderdaad komt door de remming van COX-2. Hiertoe werd een model gebruikt waarbij muizen dikke darmchirurgie ondergingen. Muizen die een erfelijke beperking hadden waardoor zij geen COX-2 konden aanmaken, hadden significant meer complicaties door een verslechterde wondgenezing. Wij vonden sterke aanwijzingen dat dit werd veroorzaakt door een verminderde capaciteit om nieuwe bloedvaatjes aan te maken. Als deze muizen prostaglandine E2 (PGE2) kregen, het belangrijkste product van COX-2 in de darm, verbeterde de wondgenezing en verminderden de complicaties. Concluderend werd in hoofdstuk 3 bewijs gevonden dat pijnstillers zoals diclofenac en aspirine vermeden dienen te worden rondom 
dikke darmchirurgie. Bovendien moet in de toekomst onderzocht worden of de toediening van PGE2 een rol kan spelen bij het verminderen van complicaties na dergelijke chirurgie.

\section{Functionele kwetsbaarheid is geassocieerd met complicaties}

In hoofdstuk 4, 5 en 6 werd de invloed van functionele kwetsbaarheid op het ontstaan van complicaties na grote maag-darmchirurgie onderzocht. Belangrijke elementen van functionele kwetsbaarheid zijn spierverval en ondervoeding. Van functionele kwetsbaarheid is met name sprake bij de oudere patiënt. Nog niet eerder werd een relatie aangetoond tussen deze kwetsbaarheid en complicaties na grote maag-darmchirurgie. In hoofdstuk 4 lieten wij zien dat deze relatie er wel degelijk is. Met name de combinatie van spierverval, ondervoeding en functionele beperkingen in het dagelijks leven zijn geassocieerd met een hoger risico op complicaties na dikke darmchirurgie. Dit geeft aan dat screening van deze kwetsbare patiënten noodzakelijk is en dat nieuwe strategieën moeten worden gezocht om deze patiënten een zo optimaal mogelijke behandeling te geven. Daarbij kan worden gedacht aan spiertraining en bijvoeding. In hoofdstuk 5 werd onderzocht of een lage hoeveelheid spiermassa de inflammatoire respons na darmchirurgie nadelig beïnvloedt. Inderdaad zagen wij een verhoogde inflammatoire respons bij patiënten met weinig spiermassa. Zeer waarschijnlijk is dit een ongunstig effect van een tekort aan spiermassa en mogelijk is dit een van de verklaringen waarom deze patiënten een hoger risico op complicaties hebben, zoals we in hoofdstuk 4 zagen. Vervolgens liet hoofdstuk 6 zien dat de mate van spierverval dat optreedt tijdens voorbehandeling met chemotherapie voorspellend is voor sterfte na slokdarmchirurgie. Deze resultaten geven in de eerste plaats aan dat met name het dynamische proces van spierverval voorspellend is voor sterfte na grote chirurgie. Daarnaast laten deze data zien dat chemotherapie, hoewel onontbeerlijk bij de behandeling van vele tumoren, een negatief effect kan hebben op de genezing van grote chirurgie. Juist deze patiënten zullen waarschijnlijk baat hebben bij maatregelen om spierverlies te voorkomen, met name in de periode van voorbehandeling met chemotherapie. 


\section{Deel 2. Diagnostische markers om complicaties op te sporen}

Het tweede doel van dit proefschrift was om markers (in dit geval eiwitten) te bestuderen die vroeg na dikke darmchirurgie een van de belangrijkste complicaties kunnen detecteren, namelijk naadlekkage. Bij een naadlekkage is de wondgenezing van de darm ontoereikend en lekken bacteriën en andere darminhoud de buikholte in waardoor patiënten erg ziek worden. In hoofdstuk 7 werden markers van darmschade en inflammatie onderzocht in het bloed van patiënten die dikke darmchirurgie ondergingen. Opvallend genoeg waren het niet de specifieke darmschade markers waarmee naadlekkage in een vroeg stadium gedetecteerd kon worden maar de markers die inflammatie in het bloed weerspiegelden. Met name de combinatie van twee verschillende markers van inflammatie (C-reactive protein en calprotectine) leidde tot vroege en accurate detectie van naadlekkage. Hiermee kon de diagnose drie dagen eerder worden gesteld dan op basis van de klinische symptomen mogelijk was. Dit is zeer relevant omdat door tijdig ingrijpen de meest schadelijke effecten van naadlekkage waarschijnlijk voorkomen kunnen worden. De toegevoegde waarde van deze combinatie van markers aan het klinische beleid zal in de toekomst verder onderzocht moeten worden in een studie waarbij deze markers worden meegenomen in de beslissing tot re-interventie.

\section{Deel 3. Darm specifieke markers bij de darmontwikkeling van kinderen}

Het derde doel van dit proefschrift was het nader onderzoeken van darm specifieke markers in het kader van darmontwikkeling op babyleeftijd, om zo beter inzicht te krijgen in de kenmerken en het ontstaan van de ernstige darmontsteking necrotiserende enterocolitis (NEC). NEC komt voornamelijk voor bij te vroeg geboren, fles-gevoede kinderen. De sterfte van deze ziekte is hoog: $20-40 \%$. Het is daarom van groot belang dat specifieke kenmerken (in het ontstaan) van NEC worden uitgezocht, om zo de diagnose en behandeling te kunnen verbeteren. Intestinal fatty acid binding protein (I-FABP), een darm specifiek eiwit, is een veelbelovende diagnostische marker voor NEC. In hoofdstuk 8 en 9 werd daarom gekeken naar de invloed van vroeggeboorte en flesvoeding op I-FABP waarden, aangezien dit de belangrijkste risicofactoren voor NEC zijn. In hoofdstuk 8 lieten wij zien dat I-FABP waarden in de darm en in het bloed toenemen naarmate het foetale ontwikkelingsstadium toeneemt in een schaapmodel. Daaruit kunnen belangrijke conclusies worden getrokken. Deze data geven aan dat I-FABP 
gebruikt kan worden als marker van het foetale ontwikkelingsstadium. Ook moet met deze verschillen rekening worden gehouden bij het stellen van de diagnose NEC. Mogelijk dienen verschillende afkapwaarden van I-FABP te worden gehanteerd naargelang de mate van vroeggeboorte. In hoofdstuk 9 werd getoond dat baby's die gevoed werden met moedermelk, een snellere ontwikkeling van de darm hadden in de eerste weken na geboorte dan baby's die flesvoeding kregen. Darmontwikkeling werd in deze studie gemeten met I-FABP. Deze resultaten bevestigen de voordelen van moedermelk en benadrukken het belang van het ontwikkelen van betere alternatieven voor of bestanddelen van flesvoeding. De effectiviteit van dergelijke nieuwe therapieën kan worden gemonitord met behulp van I-FABP.

\section{Deel 4. Markers voor diagnose, behandeling en vervolgbeleid bij NEC}

Het vierde en laatste doel van dit proefschrift was het onderzoeken van de bruikbaarheid van darm specifieke markers bij de diagnose, behandeling en follow-up van NEC. Eerder werd door onze onderzoeksgroep aangetoond dat NEC nauwkeurig kan worden gediagnosticeerd met I-FABP bij kinderen bij wie, op basis van klinische symptomen, gedacht wordt aan NEC. In hoofdstuk 10 werd deze groep onderzochte kinderen uitgebreid en werd gevonden dat de combinatie van I-FABP met calprotectine in de ontlasting van deze kinderen (hetgeen specifiek is voor darminflammatie) een nog betere diagnostische waarde gaf. Vervolgens werd in hoofdstuk 11 ontdekt dat de inflammatoire marker serum amyloid A (SAA) in de urine van kinderen met NEC gebruikt kan worden om degenen met een ernstige vorm van NEC te onderscheiden van kinderen met een minder ernstige vorm van NEC. Dat betekent dat SAA kan worden gebruikt om de beslissing te nemen welke kinderen geopereerd moeten worden, namelijk degenen met ernstige NEC. In hoofdstuk 12 tenslotte, werd onderzocht of I-FABP kan bijdragen aan de beslissing om de orale voeding opnieuw te starten bij kinderen die werden behandeld vanwege NEC. Het stoppen van de orale voeding hoort standaard bij de behandeling van NEC en het moment waarop deze voeding opnieuw wordt gestart is cruciaal: te vroeg starten kan een terugval betekenen en te laat starten kan tot groei- en ontwikkelingsproblemen leiden, met mogelijke problemen bij voeding door infusen. Wij toonden aan dat hoge I-FABP waarden op het moment van herintroductie van orale voeding correspondeerden met een slechte uitkomst. 
Met het moment van opnieuw oraal voeden, dient dus te worden gewacht totdat de I-FABP waarde laag is. Vervolgonderzoek moet aantonen of bij een dergelijke strategie de uitkomst van NEC behandeling verbetert. 

Dankwoord 
'I was working on the proof of one of my poems all the morning, and took out a comma. In the afternoon I put it back again.' Oscar Wilde

$\mathrm{Nu}$ is het tijd voor het belangrijkste gedeelte van dit boek, daar ben ik mij bewust van. Niet alleen omdat alle grote filosofen van deze aarde, van Lao-Tse tot Johan Cruijff erkennen dat je enkel met de hulp van anderen succes bereikt. Ook omdat dit het enige hoofdstuk is dat de meesten zullen lezen. Vandaar de komende lofzang.

Allereerst mijn promotoren, prof. von Meyenfeldt en prof. van Heurn.

Beste prof. von Meyenfeldt, beste Maarten. Het begon allemaal met een potje geld om naadlekkages te voorkomen. Even een RCT'tje, dat is uiteindelijk 4 jaar geworden, maar wel een proefschrift! Het was niet zo dat ik de deur bij je plat liep, maar ik heb enorm veel van je geleerd, zowel inhoudelijk als qua mentaliteit, je kalmte is bewonderenswaardig. Maar het meest staat me toch wel dat ene geniale "Dennis Bergkamp" moment bij, toen je bedacht dat het wel eens aardig zou zijn om naar sarcopenie te gaan kijken. Drie papers, alleen al in dit boekje! De afgelopen tijd is zwaar voor je geweest en toch stond je voor me klaar als er echt zaken geregeld moesten worden. Ik wens jou en Wilma veel gezondheid en geluk toe. Dank voor alles.

Beste prof. van Heurn, beste Ernst. Niemand kijkt zo snel papers na als jij. Dat was bijna irritant, want dan had ik het net afgestreept van mijn to-do lijst en dan kon ik weer aan de bak. Dankzij jou en de doordenderende NEC-trein had ik snel mijn eerste publicaties en dat is een supergevoel als beginnende AIO. Je had altijd scherpe nieuwe ideeën en ik heb ook veel geleerd van je kennis van de Engelse schrijftaal. En als er samples geanalyseerd moesten worden dan trok je weer een blik semiartsen open. Mooie momenten natuurlijk op de kinderchirurgische congressen in Barcelona en Rome. Pizzaatjes, (veel) gnocchi en schelden op het Nederlands elftal. Heerlijk. Veel succes in Amsterdam! Ik hoop dat we de NEC-lijn en alle spin-offs nog lang kunnen voortzetten.

Dan mijn copromotor, dr. Poeze. Beste Martijn, wij echte Muidenaren zijn recht door zee, dapper en bovendien extreem gespierd. Dat moet ook wel als je uit een stadje komt dat voortdurend ten prooi valt aan plunderingen en belegeringen. Weet je nog dat we met onze blote vuisten staal moesten uitharden? Ondanks die bagage heb ik je hulp vaak genoeg nodig gehad. Jij was toch het eerste aanspreekpunt 
en ik kon altijd op je terugvallen. Je hebt me daarnaast veel vrijheid gegeven, waar ik je heel dankbaar voor ben. Eigenlijk zou ik me puur met hemodynamiek en perfusie gaan bezighouden en uiteindelijk resulteerde het in dit boekje. Als er maar een goed plan achterzat, vond je het prima. Geweldig, die relaxedheid. We zullen elkaar de komende jaren gelukkig nog geregeld gaan zien.

De leden van de beoordelingscommissie wil ik graag bedanken voor het beoordelen van dit proefschrift, prof. Heineman, prof. Fearon, dr. Bouvy, dr. Lenaerts en in het bijzonder prof. Dejong. Beste Kees, de dag van de promotie van Simon Dello was gezien alle omstandigheden een heel bijzondere. Ik wil je graag bedanken voor de manier waarop je dat benadrukte in de laudatio. Echt een kippenvelmomentje, wat een mooie dag was dat!

Dan een speciale plek voor dr. Derikx. Beste Joep, er was geen plek meer in het promotieteam, maar wat mij betreft hoor je daar wel bij. Voor een groot deel dankzij jouw ongebreidelde enthousiasme en wetenschappelijke inzichten is dit boekje tot stand gekomen. Rebuttalen, samples uitmelken, statistische handigheden, we hebben alle trucs uit de kast getrokken. Wie schrijft die blijft! Naast Jeanne van afdeling 11 in het Atrium ben je tot nu toe de enige persoon die ik ken die in een ziekenhuis opbelt en zegt "met mij hè", waardoor degene die gebeld wordt altijd even moet nadenken. Goed trademark. We hebben veel gelachen op congressen en bij het avondenlang editen van promotiefilmpjes (als we weer dachten dat het format bedenken het meeste werk was). Bedankt dat ik mocht solliciteren en ben toegelaten tot het illustere wokgenootschap en indirect dus bedankt voor de Ring of Fire. Je staat bekend om het verzinnen van bijnamen en ik dank je dan ook voor Kostanios en Kostandré (Rieu). Je bent een topgozer en ik hoop dat je je draai gaat vinden in Amsterdam. Het is alleen jammer dat je liever wijn dan bier drinkt en dat je voor Fortuna bent.

Beste dr. Hoofwijk, beste Ton, allereerst bedankt voor de kans die je me hebt gegeven om mezelf in het chirurgische vak te bewijzen en voor je steun bij de sollicitatie voor de opleiding. Dank ook dat we een groot deel van de studies uit dit proefschrift bij Sittardse patiënten mochten uitvoeren. Tot slot wil ik nog even je uitspraak "de anus is een uiterst delicaat orgaan" aanhalen. 
Beste dr. Sosef, beste Meindert, van jou komt de gevleugelde uitspraak "promoveren is een soort initiatierite: eerst word je met natte takken op je zak geslagen, maar daarna hoor je erbij”. Dank je dat ik de data die in Heerlen zijn verzameld voor mijn onderzoek mocht gebruiken. Ik ben ervan overtuigd dat de komende jaren vette jaren worden, mijn start als AIOS in Heerlen was in ieder geval al super.

Dr. Stoot, beste Jan, ook jij hebt een nietsontziend enthousiasme waar velen een voorbeeld aan kunnen nemen. Alles onder de 30 lopende projecten is wat jou betreft niet te veel. Tijd zat in de periferie. Dank voor alle tijd die je voor me hebt vrijgemaakt, je hulp bij de Sittardse projecten en de avondvullende research meetings met de F(unctional) C(ompromise) Limburg taskforce bij jou thuis. Qua uren investering van onze kant zou je zeggen $100 \%$ subsidie garantie... Dank ook aan Patricia voor de catering en voor het kritisch reviseren van mijn samenvatting. Ik kijk uit naar de volgende keer dat je jezelf weer transformeert in Johnny Punch en op de bar gaat zitten. BAM!

Beste prof. Kramer, beste Boris, als neonatoloog ben je vanaf het begin zeer actief bij de NEC studies betrokken geweest. Bedankt voor je adviezen en hulp bij de stukken in dit boekje. Met een joviaal, nogal Duits klinkend "Kostannnn, hoe is het met jouwww", pretoogjes en een aanstekelijke schaterlach, stond de deur altijd wagenwijd bij je open. Ik hoop dat we nog vele jaren kunnen samenwerken.

Graag wil ik ook alle andere chirurgen uit Maastricht, Sittard en Heerlen bedanken die hebben meegewerkt aan de studies in dit proefschrift, zonder jullie inspanningen en adviezen was dit nooit gelukt. Geerard Beets, Nicole Bouvy, Karel Hulsewé, Steven Olde Damink (nee ik ga niet Parijs-Roubaix met je fietsen, dat is ziek), super bedankt!

Henriëtte Willigers, hoe vaak hebben we niet gebrainstormed over de hemodynamiek studie. We zijn zelfs naar Londen gegaan voor een Mastery of CardioQ cursus bij dr. Singer himself. Bedankt voor alles en bedankt voor de intraveneuze prehydratie voor carnaval. 
Dan het UMC Utrecht team, prof. Van der Zee, dr. Brouwers en Mayko Louwers, hartelijk bedankt voor het opbouwen en in stand houden van het NEC onderzoek daar. Zonder de inclusies in Utrecht en jullie adviezen op zowel praktisch als wetenschappelijk gebied waren de NEC studies in dit proefschrift nooit zo mooi geworden. Bernadette van Acker en Prapto Sastrowijoto, dank voor jullie wetenschappelijke suggesties en hulp bij het verkrijgen en opslaan van plasma en weefsel samples in de Sittardse studies. Jullie inzet is van grote waarde geweest.

Prof. Buurman, beste Wim, ik heb veel van je geleerd. Jouw kritische visie op wetenschappelijk gebied is uniek, van de methodologie tot het schrijven van stukken. Mijn promotietraject is anders gelopen dan wij beiden gedacht hadden. Desalniettemin bedankt voor de leerzame momenten.

Mo "de berber" Hadfoune, eerlijk is eerlijk, jij hebt mij ontdekt. En niet op basis van mijn kuuperskwaliteiten maar mijn karakteristieke sleepschot. Dat een topscout uit Woensel zulke waardeloze teams kan maken met obscure Afrikanen en afgedankte Brazilianen. Je hebt de taal van de straat, de Mocro Flavour en de swagger naar een lab gebracht waar hutspot het meest exotische gerecht was, je weet zelf. We hebben uren volgezwetst over werkgerelateerde maar vooral niet werkgerelateerde zaken. Met name onzin (gelukkig), maar ook af en toe serieuzere onderwerpen zoals de baardmannen en geopolitiek. Jammer (en onverstandig) dat je voor PSV bent, wordt toch weer billenknijpen met slechts 10 punten voorsprong en nog 5 wedstrijden, je weet het nooit...

Tim Wolfs, supercoach Sjeng, ongelofelijk dat iemand op basis van pure mazzel zo vaak coach van het jaar kan winnen. Toch verdenk ik je ervan dat je nachtenlang hebt liggen bellen met je goede vriend Sef Vergoossen en allerlei spelermakelaars om aan informatie te komen, smeerlap. Gelukkig heb je er geen grijze haren van gekregen. Bedankt voor de top samenwerking, de avondjes MVV (maar je zult begrijpen dat ik voorlopig geen seizoenskaart meer haal), Wokking with the Boys, je beef met Dirk, en ga zo maar door. Vaan Pèjsie (mèt ut rejtahbein), tot weer uns get is!

Joep Grootjans, Grandjean, ontzettende professor. Gelukkig ben je in alles wat je doet een fanatiek kereltje, dat heeft ons in Chicago geen windeieren gelegd! Lekker clubben en fistpumpen, de clubdeuren achter ons dichttrekken en om $8 u$ 
gewoon weer acte de présence geven om vervolgens uiterst geboeid naar een willekeurige Chinees te luisteren. En daarna deze cyclus herhalen. Ik ken niemand die zo rigoreus dingen kan aanpakken, van whisky verzamelen tot rondjes lopen, echt respect! Bedankt voor al je hulp en adviezen.

Dirkster ouwe pikkepuuner, bedankt voor de super samenwerking. Jij stond altijd voor me klaar als er iets moest gebeuren. Op het platteland zijn ze natuurlijk gewend om de handjes uit de boerenkiel te steken, maar ik ben blij dat je je riek aan de wilgen hebt gehangen in ruil voor de pipet en de muizenguillotine. Altijd lachen als je weer eens met een verhaal op de proppen kwam waarbij mij de clou nooit helemaal duidelijk was, maar waarbij je verontwaardiging de belangrijkste bottomline was. Veel succes met je eigen promotie en opleiding, komt zeker goed!

Liliane, ik kan de silent $\mathrm{M}$ in Mpabanzi nog steeds niet goed uitspreken maar je bent een topper! Na het werk samen "affietsen" inclusief natuurlijk het bespreken van alles en iedereen, altijd gezellig. Jij bent de enige vrouw die ik ken waarbij het niet irritant is als ze over voetbal praat. Je hebt een behoorlijke dosis positieve energie bijgedragen aan het leven op het lab, bedankt!

$\mathrm{AC}$, we can't all be princesses, someone has to clap when I go by. Ik weet niet precies waarom dat op jou van toepassing is, want je werkt keihard. Zo'n enthousiast en spontaan meisje met een goed gehalte springerigheid wil iedereen wel als student (en opvolgster!). Je hebt jezelf natuurlijk onsterfelijk gemaakt door op een chirurgisch congres te zeggen "I'm really interested in Cox-2" (fonetisch uit te spreken, voor de kijkers en luisteraars thuis). Succes met alles en we worden zeker nog collega's!

Basje Boonen, ook nooit te beroerd om hulp te bieden of eindeloos over voetbal te zwetsen, alleen meestal over de verkeerde club helaas. Wordt het een platte kar dit seizoen, of toch weer een March of Sadness? We zullen zien. Dennis Meesters, de Dove Whisperer, wandelende Flabber-encyclopedie. Je hebt jezelf losgemaakt uit de Schinveldse posse, geen ongevaarlijke stap. "I tried to get out, but they pulled me back in." Merciekes voor de gezelligheid. Annemarie, zonder jou was dit nooit gelukt. Alle ELISA analyses waren van onschatbare waarde. Als dit boek een bestseller wordt, waar ik wel vanuit ga, dan doe jij mee in de winst! Beschouw jezelf dus als financieel onafhankelijk. Ruben Visschers, de duo-penotti 
van mafkees en relaxedheid, samen vertolken wij denk ik 90\% van de (hoofd) rollen in promotiefilmpjes. Bedankt voor alle humor en voor je mentorschap van mijn eerste schreden in de wondere wereld van de Chirurzjie. Givan, topcongres in Barcelona, inclusief summervibes op het strand met lauwe blikken bier! Charlotte, collega's van het eerste uur op het lab, slap ouwehoeren over tropische vakantieoorden of zelfs hypotheken. En natuurlijk mede-organisator van het beste labuitje evah. Mechteld, leuk dat we weer collega's zijn, kunnen we mooi samen saaie cursussen bezoeken. Da kan toch helemaal nie Timur! Tim van Smaalen, goedlachs mannetje, koning van het hakje achter het standbeen, hopelijk kunnen we het voetballen nog een tijd voortzetten.

Alle andere (oud)collega's van het lab die ik nog niet genoemd heb, bedankt voor de mooie tijd. Aart, Bas Hanssen, Bart, Caroline, Claire, David, Edgar, Eva, Filip, Frank, Frans, Freek, Froukje, Geertje, Guy, Hans, Inca, Iris, Irene-Fleur, Irma, Jacco, Johanne, Joyce-Manyi, Maartje, Marlou, Kaatje, Kevin, Kim van Mierlo, Kim van Wijck, Kiran, Kirsten Huntjens, Kirsten van der Beek, Leontine, Lori, Luc, Maarten (beter bekend als Snoeijs(haan)), Marc Schreinemacher, Mark de Wolf, Martine, Milou, Nina, Pieter, Ralph, Rob, Robbert-Jan, Ruben Vogels, Rutger, Sander, Selwyn, Sofia, Tiara, Tim Lubbers, Toine en Yee-Lai. Daarnaast ook alle Sittardse collega's dank voor de mooie periode. De collega's in Heerlen, wat een leuke groep, ik heb zin in de komende jaren.

Jeroentje van Vugt en Juul Tegels, boys en hooligans van de FC Limburg werkgroep. Bedankt voor al het belangrijke werk van samples verzamelen, database bouwen, analyseren etc. En dan vergeet ik nog de studentes Joany en Loes, bedankt voor het roeren in de poepsamples, zo ben ik ook nog ooit begonnen.

Christine, super bedankt voor de hulp bij het ontwerpen van de kaft van dit boekje, dat had ik nooit zelf gekund.

Frankie (de Boer), bedankt voor de derde ster en de serie kampioenschappen daarna. Bedankt voor je arrogante norse kop en je triomfantelijke lachje.

De volgende alinea slaat nergens op, maar hij is wel lekker lang en ik ga mijn vrienden toch bedanken voor hun wetenschappelijke input en bijdrage aan dit boekje. Daveje Devens, geven of ontvangen, dat is de vraag en ik weet wat jouw 
antwoord is. Lullen als brugman is zowel letterlijk als figuurlijk je vak en je kunt 's nachts wakker gemaakt worden voor I save the day. Voor meditatieve doeleinden bezochten we hijskranen en leegstaande fabrieken. Ik heb hier veel inspiratie uit geput. Boze tongen beweren dat je 100\% pure gay/homosexual bent, maar dat is niet zo. De E-town Barracuda, samen met ondergetekende koning van de after. Wij laten ons graag in Oost-Europa in de nek slaan. Voor een fles bacardi en twee flessen cola is ons motto: als er een gat in zit, dan draaien we het. BaKo powerrr. Das FAKKING angstgegner, collega freak. We hebben de zin van onzin tot kunst verheven. Blijkbaar is de wereld nog niet klaar om dit te begrijpen. Maar we geven ze gewoon de middelvinger als het moet. Dat zal ze leren. Kace als onderdeel van de misty dogs, Keizer van de Lounge, jij vreet een blok brie in een keer op en schaakt daarnaast een keer per jaar. Een unieke combinatie. Jman, lekker sappie hoor hee! Ouwe Rottedammejt, mot je een spettert op je kerstboom dan? Op nog veel vakanties met die druktemakers van koters van ons! Nelissenkoerser / Bad Motherfucker / The Praying Mantis: de koers is van ons (en van de G-chef met zijn decadente liflafjes). Je hebt met je markante klapkop besloten dat je geen blad meer voor de mond neemt. Dat doet vermoeden dat je dat eerst wel deed. Rijst (sushi, rijstevlaai) is niks voor jou, tenzij opgewarmd. Zoet is prima, zolang het geen zoetigheden betreft. Je taktiek van wachten in het Pothuis begint langzaamaan zijn vruchten af te werpen en de MILFs komen als vliegen op de stront op je af. Jean "Massimo" Bessems, roadtrip buddy, immer geradeaus, dat waren tijden. Mooie gifgroene broek had je toen, waar is die eigenlijk? Sjtefke, Geo, Sandah (in het circuit beter bekend als Billy the Clift) bedankt voor de inspirerende vakantie op Camping La Ballena Alegre aan de Costa Brava. Ik denk dat in discotheek Moby Dick de basisprincipes voor dit proefschrift zijn gelegd. Waarom ook niet eigenlijk. Eveje, de aaibaarheidsfactor in hoogsteigen persoon. We zijn zelfs nog even collega's geweest! Het is een publiek geheim dat jíj eigenlijk degene bent die keihard is en niet die kale hufter. En als het erop aan komt is "money no point". De Goebster, liefkozend De Tank genoemd door je broertje (die gek is, overigens). Inmiddels heb je het taphangen ingeruild voor Aperol Spritzkes. Hoedje erop en mevrouw is mondaine. Je probeert mijn vrouw af te pakken, dat bevalt me niet. Lindaatje, BL, van doorhalen in de Celebrasie tot middagdutjes aan het zwembad als de kinderen slapen. Wie had dat gedacht. Ik in ieder geval niet toen we rosé bubbels op jouw kamer zaten te drinken. Dat klinkt ook behoorlijk gayhomosexual trouwens, nu ik het zo bedenk. Sampje B, wij bepalen wat hip is. Zoals bijvoorbeeld donderdagavond Alla avond, wat is uitgegroeid tot een ware hit. Wat 
Youp van 't Hek kan met Buckler bier, dat doen wij met trends. Als wij niet willen dat John Tinnic als drankje bij het grote publiek doorbreekt, dan flopt het door een simpele spitsvondige opmerking. Hke, lady in pink, de schakel tussen mij en mijn aanstaande echtgenote. Oneindige dank daarvoor! Nog iets anders: ongelofelijk hoeveel geluid er uit zo'n klein meisje kan komen. Party Jane, de showdog van het gezelschap. Sushi extravaganza is jouw middle name. Altied in veur e grepke en als het je te heet onder de voeten wordt, kun je altijd nog van de bank af glijen. De mannen van T.S.G. 51 ${ }^{\circ} 45^{\prime}$, bedankt voor de prachtige studententijd, klootschieten en assholen met spekjes in je neus. En tot slot iedereen die ik nog niet genoemd heb: Anouk, Denise en Frank (laat je zak zien!), Lieke B, Lieke N, Liska, Nick, Sharon, Wouter, Ylaine en Jeroen. Allemaal bedankt voor de bruikbare suggesties en het kritisch reviseren van dit boek.

Dan de paranimfen, Simon Dello en Jonas Göbbels.

Simon, ik geloof dat we elkaar helemaal in het begin maar freaks vonden, maar al snel werden we dikke matties op het lab. Dat kwam voornamelijk omdat we over alles wel een mening hadden en meestal dezelfde, waarbij de conclusie dan altijd was dat we het zelf beter konden. Mooi ook de fietstochtjes samen, waarbij jouw fanatisme er soms voor zorgde dat je jezelf compleet opblies met amper 10 kilometer op de teller. Geen tussenweg bij jou. Volle gaas of niet. Gelukkig ben ik precies hetzelfde en heb ik me daarom niet gewaagd aan rennen met jou, ik heb namelijk geen zin om te verliezen. Ik heb enorm respect voor jouw doorzettingsvermogen en veelzijdigheid. Geen eenvoudige opdracht om in de cardiologische periferie op een chirurgisch onderwerp te promoveren, maar je hebt het geflikt! Ik hoop dat je blijft schilderen en bizarre fietsen blijft bouwen. Het is jammer dat we geen directe collega's worden, maar ik heb daarvoor in de plaats een maat voor het leven overgehouden.

Jonas, Splitting Joe, al meer dan tien jaar een van mijn allerbeste maten. Ik ben trots dat je naast me staat. We hebben veel mooie dingen meegemaakt en je stond altijd klaar om een potje te poolen en te lachen maar ook als ik ergens mee zat. Je doet net alsof je het meest geniet van een "goed glas" rode wijn in je schommelstoel bij het haardvuur, maar ik weet dat je diep in je hart het liefst wil atten met de gasten en elkaar tegen de kloten meppen. Dat verandert gelukkig ook niet nu je huisarts bent. En ook niet als je een bruine leren tas en een motor koopt. Shotgun, trouwens. 
Lieve familie en in het bijzonder oma, zo oud, nog zo gezond en nog even lief. De levende legende van de worstenbroodjes; en nu ben je zelfs overgrootmoeder! Ook lieve schoonfamilie, Joost en Joke en de rest, als ik wilde kon ik altijd voor raad en daad bij jullie terecht.

Mijn lieve ouders, papa en mama, dank jullie wel voor de steun die ik mijn hele leven van jullie heb gekregen bij alles wat ik wilde doen. Jullie zullen vast ettelijke grijze haren hebben gekregen van mijn gedrag aan het einde van de middelbare school en getwijfeld hebben of het allemaal wel goed zou komen. Ik denk dat je pas later beseft hoe belangrijk de onvoorwaardelijke liefde en steun van je ouders is. Justus en Emma, ik ben trots op jullie, wat jullie allemaal klaarspelen op werken studiegebied, maar ook daarbuiten. Dan geef ik toch maar toe dat mijn jongere broertje harder fietst, ai ai ai... En de Emster, kleine zusjes worden groot. Wat een leventje heb jij opgebouwd in Utreg. Hopelijk kies je een niet al te exotische vent! Ook wil ik jou nog noemen, Nina, want jij hoort er ook bij. Wat zou er van jou geworden zijn? Ik kan me je niet meer goed herinneren en toch zal ik je niet vergeten, want jouw bestaan heeft mij mede gevormd tot wie ik ben.

Lieve lieve Aleida, mijn aanstaande, wat is het leven mooi met jou. Als we willen kunnen we de hele nacht aan de keukentafel zitten met zijn tweeën en raken we nog niet uitgepraat. Ik kan je niet meer missen, ook al wil je altijd een hapje van mijn hamburger en koop je flesjes gezichtsreiniger van 40 euro het stuk. Jij bent mijn zonnetje en kan me vrolijk maken als ik dat niet ben, motiveren als ik geen zin heb. Dit boekje is dus grotendeels aan jou te danken. Hoe jij moeder en psychiater zijn combineert met promoveren is een heel groot applaus waard. Dank je voor alles. Tenslotte Nina en Max, jullie zijn het kostbaarste bezit en maken al het andere relatief. Ik ben dankbaar dat jullie er zijn, gezond en wel.

“Onskuldig ogies en voetjies en handetjies

Wie weet hoe ver moet my kleintjie nog ganetjies

Ver deur die wêreld se kronkels en gangetjies

Bly tog maar kleintjies en bly dit maar langetjies

Bly maar by moedertjie, kindlief se hoedertjie

Slaap in haar arrempies, saggies en warrempies" 


Scientific output 
de Haan JJ, Thuijls G, Lubbers T, Hadfoune M, Reisinger K, Heineman E, Greve JW, Buurman WA. Protection against early intestinal compromise by lipid-rich enteral nutrition through cholecystokinin receptors. Crit Care Med 2010; 38:1592-7.

Reisinger KW, Derikx JP, Kramer BW. Need for biomarkers to differentiate between NEC and sepsis. J Clin Invest 2010, E-letter.

Dello SA, Reisinger KW, van Dam RM, Bemelmans MH, van Kuppevelt TH, van den Broek MA, Olde Damink SW, Poeze M, Buurman WA, Dejong CH. Total Intermittent Pringle Maneuver during Liver Resection Can Induce Intestinal Epithelial Cell Damage and Endotoxemia. PLoS One 2012; 7:e30539.

Reisinger KW, van der Zee DC, Brouwers HA, Kramer BW, van Heurn LW, Buurman WA, Derikx JP. Non-invasive measurement of fecal calprotectin and serum amyloid A (SAA) combined with intestinal Fatty Acid Binding Protein (I-FABP) in necrotizing enterocolitis (NEC). J Ped Surg 2012; 47:1640-5.

Dello SA, Lodewick TM, van Dam RM, Reisinger KW, van den Broek MA, von Meyenfeldt MF, Bemelmans MH, Olde Damink SW, Dejong CH. Sarcopenia negatively affects preoperative total functional liver volume in patients undergoing liver resection. HPB (Oxford). 2013 Mar;15(3):165-9.

Reisinger KW, Derikx JP, Thuijls G, van der Zee DC, Brouwers HA, van Bijnen AA, Wolfs TG, van Heurn LW, Buurman WA, Kramer BW. Noninvasive measurement of intestinal epithelial damage at time of refeeding can predict clinical outcome after necrotizing enterocolitis. Pediatr Res. 2013 Feb;73(2):209-13.

Reisinger KW, de Haan JJ, Schreinemacher MH. A word of caution before implementing ketotifen for gastrointestinal transit improvement. World J Gastroenterol. 2013 Jul 21;19(27):4445-6

Reisinger KW, Kramer BW, Van der Zee DC, Brouwers HA, Buurman WA, van Heurn LW, Derikx JP. Non-invasive serum amyloid A (SAA) measurement and plasma platelets for accurate prediction of surgical intervention in severe necrotizing enterocolitis (NEC). PLoS One. 2014 Mar 6;9(3):e90834

Reisinger KW, van Vugt JL, Tegels JJ, Snijders C, Hulsewé KW, Hoofwijk AG, Stoot $\mathrm{JH}$, von Meyenfeldt MF, Beets GL, Derikx JP, Poeze M. Functional compromise reflected by sarcopenia, frailty and nutritional depletion predicts adverse postoperative outcome after colorectal cancer surgery. Ann Surg. 2014 Mar 19 
Reisinger KW, Elst M, Derikx JP, Nikkels PG, de Vries B, Adriaanse MP, Jellema RK, Kramer BW, Wolfs TG. Intestinal fatty acid binding protein (I-FABP): a possible marker for gut maturation. Pediatr Res. 2014 Sep;76(3):261-8

Reisinger KW, Poeze M, Hulsewé KW, van Acker BA, van Bijnen AA, Hoofwijk AG, Stoot JH, Derikx JP. Accurate prediction of anastomotic leakage after colorectal surgery using plasma markers for intestinal damage and inflammation. J Am Coll Surg. 2014 Oct;219(4):744-51

Reisinger KW, Derikx JP, Stoot JH, Poeze M. Reply to letter: "Computerized tomography based diagnosis of sarcopenia?” Ann Surg. 2014 Jul 28.

Reisinger KW, de Vaan L, Kramer BW, Wolfs TG, van Heurn LW, Derikx JP. Breast feeding improves gut maturation compared to formula feeding in preterm babies. J Pediatr Gastroenterol Nutr. 2014 Aug 7

Van Vugt JL, Reisinger KW, Derikx JP, Boerma D, Stoot JH. Improving outcomes in oncological colorectal surgery. World J Gastroenterol. 2014 Sep 21;20(35):1244512457

Reisinger KW, Bosmans JW, Uittenbogaart M, Alsoumali A, Poeze M, Sosef MN, Derikx JP. Loss of skeletal muscle mass during neoadjuvant therapy predicts postoperative mortality in esophageal cancer surgery. Ann Surg Oncol. In press

Reisinger KW, Derikx JP, von Meyenfeldt MF, Hulsewé KW, Stoot JH, Poeze M. Sarcopenia is associated with an increased inflammatory response to surgery in colorectal cancer. Submitted

Reisinger KW, Willigers HM, Jansen J, Buurman WA, von Meyenfeldt MF, Beets GL, Poeze M. Doppler guided goal-directed fluid therapy increases intestinal perfusion in colorectal surgery. Submitted

Reisinger KW, Schellekens DH, Derikx JP, Grootjans J, von Meyenfeldt MF, Poeze M. Cyclooxygenase-2 (cox-2) is essential for colorectal anastomotic healing. In preparation 

Curriculum vitae 
Kostan Reisinger was born on the 16th of October 1985 in Muiden. He grew up in Geulle and attended high school at the Jeanne d'Arc College in Maastricht from 1997 to 2003. After graduating Cum Laude, he received his medical training at the Maastricht University. He graduated in 2010, and worked as a PhD-student at the Department of Surgery under supervision of Prof. dr. M.F. von Meyenfeldt, Prof. dr. L.W.E. van Heurn, and Dr. M. Poeze, which resulted in the present thesis. In 2014, he started as a surgical resident at the Orbis Medical Centre in SittardGeleen (supervision Dr. A.G.M. Hoofwijk). In 2015, he started working at the Atrium Medical Centre in Heerlen (supervision Dr. M.N. Sosef), which is part of his surgical training at the Maastricht University Medical Centre (under supervision of Prof. dr. L.P.S. Stassen). With his fiancé Aleida Frissen he has two children, Nina and Max. 


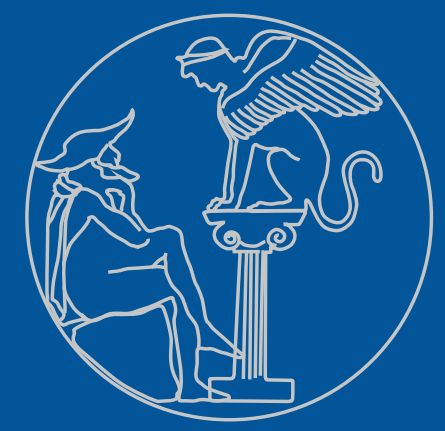

